\title{
Contribuições à teoria dos Grupos Especiais
}

\author{
Hugo Luiz Mariano
}

\author{
Tese apresentada \\ ao \\ Instituto de Matemática e Estatística \\ da \\ Universidade de São Paulo \\ para a obtenção do grau de Doutor \\ em \\ Matemática \\ Área de concentração: Matemática \\ Orientador: Prof. Dr. Francisco Miraglia
}

Durante a elaboração deste trabalho o autor recebeu apoio financeiro do $C N P q$ 


\section{Contribuições à teoria dos Grupos Especiais}

Este exemplar corresponde à redação final da tese devidamente corrigida e defendida por

Hugo Luiz Mariano

e aprovada pela comissão julgadora.

São Paulo, 26 de fevereiro de 2003.

Banca examinadora:

- Prof. Dr. Francisco Miraglia Netto (Orientador) - IME-USP

- Prof. Dr. Flávio Ulhoa Coelho - IME-USP

- Prof. Dr. Walter Alexandre Carnielli - CLE-UNICAMP

- Profa. Dra. Ítala Maria Loffredo D'Ottaviano - CLE-UNICAMP

- Prof. Dr. Edward Hermann Haeusler - PUC-RJ 


\section{Dedicatória-Agradecimentos}

Aos meus pais João (Zé) e Beth, minha irmã Dé e minha garota Rosinha(Nenê), pelo carinho e retaguarda de vocês, que tornaram felizes estes, por vezes difíceis, momentos de elaboração de uma tese.

A todos meus amigos, dentro ou não da Universidade, com quem mais convivi nestes anos: Lola (e Igor), Beth, Fernando, Zé Antônio, Sui e tantos mais.

Aos colegas, professores e funcionários do IME-USP, particularmente aos interessados na área de Lógica, havendo portanto uma interação mais frequente. Entre eles: o Odilon, Ricardo Bianconi, Claus, Piotr e também o Marcelo Coniglio e Andreas e os participantes do seminário de Lógica conjunto IME-CLE. Em especial aos professores-amigos Bianconi e Odilon que partiparam de minha orientação.

Reservo uma palavra de gratidão muito especial para o Chico Miraglia, meu orientador nesta tese. O Chico foi um exemplo extremo tanto de profissionalismo quanto de entusiasmo pela Matemática: aprendi muito com ele e espero ter entendido como deve-se fazer Matemática! Chico, minha dívida com você é irresgatável!

Muito Obrigado a todos vocês!

Hugo Luiz Mariano 


\section{Resumo}

Este trabalho é um desenvolvimento de certos aspectos lógico-categoriais da teoria dos Grupos Especiais - uma formulação em linguagem de primeira ordem da teoria algébrica das formas quadráticas. Em estudo estão os grupos especiais profinitos - certos tipos de limites que a categoria dos grupos especiais admite. Construímos um funtor da categoria dos grupos especiais reduzidos e seus morfismos correspondentes (RSG) na categoria constituída dos grupos especiais profinitos e dos morfismos contínuos de grupos especiais $\left(\mathbf{R S G}_{p f}\right)$. Verificamos que esse funtor merece o título de "funtor envoltória profinita" : é o adjunto à esquerda da inclusão $\mathbf{R S G}_{p f} \hookrightarrow$ RSG. Analisamos o comportamento deste funtor por construções categoriais: mostramos que este preserva quocientes e monomorfismos completos. Apresentamos a noção de morfismo de grupos especial que reflete subforma e mostramos que a transformação natural que é unidade desta adjunção é constituída por morfismos desta espécie. Identificamos esta flecha no caso de um grupo especial proveniente de uma álgebra booleana com o morfismo de álgebras booleanas que gera a topologia no espaço de Stone correspondente e exploramos esta identificação. 


\section{Abstract}

This work is a development of some logical-categorial aspects of the theory of Special Groups - a first order presentation of the algebraic theory of quadratic forms. We study the Profinite Special Groups - certain kinds of limits that the Special Groups category has. We build a functor from the category RSG of reduced special groups with $S G$-morphisms to the category $\mathbf{R S G}_{p f}$ of profinite reduced special groups with continuous $S G$-morphisms. We verify that this functor deserves the title of "profinite hull functor": it is the left adjoint of the inclusion $\mathbf{R S G}_{p f} \hookrightarrow \mathbf{R S G}$. We analyze the behavior of this functor by categorial constructions : we show that it preserves quotients and complete embeddings. We present the concept of a subformreflecting morphism between special groups and we show that the natural transformation that is the unit of such adjunction is composed by this kind of morphisms. We identify this arrow in the case of the special group that came from a boolean algebra with the boolean algebras morphism that generates the topology on the respective Stone space and explore such identification. 


\section{Sumário}

Dedicatória-Agradecimentos 5

$\begin{array}{lr}\text { Resumo } & 7\end{array}$

$\begin{array}{ll}\text { Abstract } & 9\end{array}$

$\begin{array}{ll}\text { Prefácio } & 15\end{array}$

1 Preliminares 19

1.1 Ordens Parciais, Filtros e Ultrafiltros . . . . . . . . . . . . . . 19

1.2 Construções categoriais . . . . . . . . . . . . . . . . . . 21

1.2 .1 Categorias e Morfismos . . . . . . . . . . . . . . . . 21

1.2.2 Funtores e Transformações Naturais . . . . . . . . . . . . . . . . . 26

1.2.3 Funtores Adjuntos e Equivalência de Categorias . . . . . . . . . . . . . 30

1.2 .4 Diagramas e Limites . . . . . . . . . . . . . . . . . . 32

1.2.5 Categorias de Diagramas . . . . . . . . . . . . . . 40

1.2 .6 Objetos Injetivos e Projetivos . . . . . . . . . . . . 43

2 Limites e Colimites de Estruturas de Primeira Ordem 45

2.1 Linguagens e Lógicas de Primeira Ordem . . . . . . . . . . . . . . . . 45

2.2 Estruturas de Primeira Ordem e seus Morfismos . . . . . . . . . . . . . . 49

2.3 Limites em $\mathbf{L}$-mod . . . . . . . . . . . . . . . . . . . . 57

2.4 Quocientes em $\mathbf{L}$-mod . . . . . . . . . . . . . . . . . 60

2.5 Colimites em $\mathbf{L}$-mod . . . . . . . . . . . . . . . . . . 61

2.6 Morfismos Puros . . . . . . . . . . . . . . . . . . 67

2.7 Estruturas Profinitas e Ultraprodutos . . . . . . . . . . . . . . 73 
3 Grupos Especiais $\quad 81$

3.1 Introdução . . . . . . . . . . . . . . . . . . . . 81

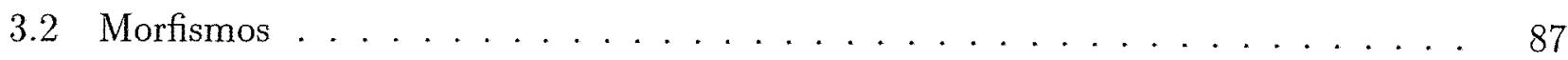

3.3 Subgrupos Saturados e Quocientes . . . . . . . . . . . . . . 94

3.4 Alguns Tipos de Grupos Especiais . . . . . . . . . . . . . . . . . . 100

3.4 Produtos, Extensões e Fans . . . . . . . . . . . . . . . . 100

3.4 .2 Corpos e Grupos Especiais . . . . . . . . . . . . . . . . 102

3.4.3 Álgebras de Boole e Grupos Especiais . . . . . . . . . . . . . . . . . 104

3.4.4 Grupos Especiais de Comprimento de Cadeia Finito . . . . . . . . . . 106

3.5 O Anel de Witt de um Grupo Especial . . . . . . . . . . . . . . . . . 110

4 A Adjunção Induzida por um SG-Morfismo $\quad 113$

4.1 A Adjunção Induzida . . . . . . . . . . . . . . . . . . . 113

4.2 Morfismos Derivados . . . . . . . . . . . . . . . 116

4.3 Sobre alguns subconjuntos de subgrupos saturados . . . . . . . . . . . . 120

5 Grupos Especiais Profinitos $\quad 125$

5.1 Grupos Topológicos e Profinitos . . . . . . . . . . . . . . . . 125

5.2 Grupos Especiais Profinitos . . . . . . . . . . . . . . . . . 130

5.3 A Envoltória Booleana de um Grupo Profinito Reduzido . . . . . . . . . . . . 149

5.4 A Envoltória Profinita . . . . . . . . . . . . . . . . 153

5.5 A Envoltória Profinita como Funtor . . . . . . . . . . . . . . . 161

5.6 Extensão ao caso Hausdorff . . . . . . . . . . . . . . . . . . 167

5.6 .1 Sobre os Grupos Especiais Topológicos . . . . . . . . . . . . . 168

5.6 .2 Sobre $\mathbf{R S G}^{\text {Haus }} \ldots \ldots \ldots \ldots \ldots \ldots \ldots \ldots$

5.6 .3 O funtor "Envoltória Especial Profinita" . . . . . . . . . . . . 171

5.6 .4 Sobre a adjunção $\mathbf{R S G}^{\text {Haus }} \rightleftarrows \mathbf{R S G}_{p f} \ldots \ldots \ldots \ldots \ldots$

$5.6 .5 \mathcal{P}$ e epimorfismos . . . . . . . . . . . . . . . 174

6 O funtor $\mathcal{P}$, Álgebras de Boole e Aplicações $\quad 185$

6.1 Introdução . . . . . . . . . . . . . . . . . 185

6.2 Subgrupos Saturados e Ideais de Índice Finito . . . . . . . . . . . . . 188

6.3 A Envoltória Profinita de uma Álgebra de Boole . . . . . . . . . . . . . . . 192 
6.4 A Preservação de Imersões Completas . . . . . . . . . . . . . . . . . . . . 199

$\begin{array}{ll}\text { Referências Bibliográficas } & 207\end{array}$

Índice Remissivo $\quad 211$ 


\section{Prefácio}

Este trabalho é um desenvolvimento de certos aspectos lógico-categoriais da teoria dos Grupos Especiais - uma fórmulação em linguagem de primeira ordem da teoria algébrica das formas quadráticas - formulada nos anos 90, apresentada em detalhes em [DM1], e que vem demons trando sua importância em diversos trabalhos, favorecendo a solução de diversas conjecturas em aberto por décadas ([DM2], [DM3] e muito recentemente a conjectura de Kahn-Hoffman) .

O objeto de estudo foi a classe dos grupos especiais profinitos - limites projetivos de sistemas cofiltrados de grupos especiais finitos. Estes foram introduzidos, por meio da linguagem dual dos espaços de ordem abstratos, em [KMS], sendo posteriormente considerados também em [Lim] e [Ast1]. Mais recentemente, sua importância expande-se com [Mar7].

Nossa abordagem foi distinta: assim como em [DM1] estuda-se como as álgebras booleanas tornam-se exemplos (muito importantes!) de grupos especiais reduzidos e que a todo grupo especial reduzido está associada naturalmente uma álgebra booleana, sua envoltória booleana, aqui, construímos o funtor envoltória profinita da categoria dos grupos especiais reduzidos e seus morfismos correspondentes (RSG) na categoria constituída dos grupos especiais profinitos e dos morfismos contínuos de grupos especiais $\left(\mathbf{R S G}_{p f}\right)$.

Detalhamos mais a estrutura do trabalho:

Estando este trabalho na interface entre lógica e álgebra (teoria das categorias) e se valendo de conceitos e métodos de ambas, decidiu-se apresentar breves introduções desses domínios da Matemática:

No Capítulo 1 constam as definições e resultados principais da teoria das categorias (as definições básicas de categoria, funtor, transformação natural, adjunção, limite/colimite,...) assim como as definições de conjuntos ordenados dirigidos, filtros, ultrafiltros,.... Acrescentamos alguns tópicos mais especializados como objetos injetivos/projetivos e categorias de diagramas e morfismos projetores.

A seguir, apresentamos no Capítulo 2 os conceitos básicos da Lógica e Teoria de Modelos de primeira ordem: as noções de linguagem, fórmulas, estruturas adequadas a linguagens e diversos tipos de morfismos entre estruturas. Adicionamos seções onde são apresentadas algumas propriedades da categoria $\mathbf{L}-\mathbf{m o d}$, a categoria das L-estruturas e L-homomorfismos. Finalizamos o Capítulo com um de nossos resultados principais: estruturas profinitas são retratos de certos ultraprodutos de estruturas finitas (2.50).

Reservamos o terceiro Capítulo para descrever brevemente as propriedades fundamentais dos grupos especiais necessárias adiante, no núcleo do trabalho. A referência básica para esta 
teoria é, naturalmente, [DM1]. Apresentamos também algumas definições e resultados novos: como a noção de morfismo que reflete subformas (3.20.(c)) e a de extensão de fórmulas existenciais positivas por formas de Pfister (3.35).

O Capítulo 4 é essencialmente técnico: nele figuram os instrumentos necessários para a construção do funtor envoltória profinita. Inicialmente apresentamos as noções de adjunção induzida (4.1) e de morfismos derivados de um morfismo entre grupos especiais reduzidos (4.4); em seguida, analisamos alguns subconjuntos dos reticulados dos subgrupos saturados de grupos especiais reduzidos e como estes se comportam pela adjunção induzida por um morfismo.

No quinto Capítulo, o principal deste trabalho, tratamos dos grupos especiais profinitos. Iniciamos verificando a existência destes limites projetivos na categoria dos grupos especiais por dois procedimentos distintos, 5.8 e 5.11 , cada um dos quais favorece novos resultados : traba lhando com primeira abordagem obtemos que todo grupo especial reduzido profinito é subgrupo especial puro de um grupo especial reduzido representável, donde satisfaz a conjectura de Marshall (5.8.(c)), e que todo grupo especial reduzido satisfaz o critério de Pfister para subformas (5.49) (resultado presente em [Ast2]); a segunda versão favorece uma abordagem mais topológica-analítica dos grupos especiais profinitos como os resultados 5.17 e 5.22 , presentes em [Lim], e nossa contribuição 5.28. Estudamos, na terceira seção, as envoltórias booleanas de grupos especiais profinitos (5.40). Construímos a envoltória profinita de um grupo especial reduzido por dois métodos distintos: um topológico-analítico (5.43), utilizando a caracterização 5.17; outro funtorial (5.51), utilizando resultados do Capítulo 4 . Verificamos que esse funtor, denotado no texto por $\mathcal{P}: \mathbf{R S G} \rightarrow \mathbf{R S G}_{p f}$, merece o título de funtor "envoltória profinita" : é o adjunto à esquerda da inclusão $\mathbf{R S G}_{p f} \hookrightarrow \mathbf{R S G}$; mostramos ainda que a transformação natural que é unidade desta adjunção é constituída por morfismos que preservam e refletem subformas. Analisamos o comportamento do funtor construído por construções categoriais: vimos que este preserva quocientes (5.54) . Na última seção do Capítulo observamos que parte importante das construções e resultados podem ser estendidos da categoria dos grupos especiais reduzidos discretos à categoria dos grupos topológicos Hausdorff que são especiais e reduzidos e morfismos contínuos (5.64 e 5.68).

Finalizamos nosso trabalho no Capítulo 6, onde discutimos a álgebra booleana profinita das partes de um conjunto (6.13) e identificamos a flecha natural originada da adjunção no caso de um grupo especial proveniente de uma álgebra booleana com o morfismo de álgebras booleanas que gera a topologia no espaço de Stone correspondente (6.18). Adiante, esta identificação foi explorada: ela nos habilitou concluir que o funtor $\mathcal{P}$ preserva e reflete monomorfismos completos (6.23).

Os comentários que seguem indicam as origens deste trabalho e apresentam algumas pers pectivas futuras:

Se por um lado grupos especiais reduzidos provenientes de álgebras booleanas são grupos especiais representáveis e grupos especiais reduzidos profinitos são sabidos apenas retratos de grupos especiais representáveis (segue que ambos satisfazem a conjectura de Marshall), por outro lado a envoltória especial profinita de grupos especiais reduzidos é uma construção "mais fina" que a de envoltória booleana: enquanto a imersão natural de um grupo especial reduzido em sua envoltória booleana preserva e reflete isometria entre formas de mesma dimensão, o mergulho canônico na envoltória especial profinita preserva e reflete a relação "ser subforma de" entre pares genéricos de formas do grupo especial de partida. 
Ambas as construções citadas podem ser entendidas como codificações de princípios locaisglobais:

Seja $G$ um grupo especial reduzido e $\varphi, \psi$ formas sobre $G$ então :

* $\varphi$ é $G$-isométrica a $\psi \Leftrightarrow$ para cada $\Delta$ subgrupo saturado maximal de $G \quad \varphi / \Delta$ é $G / \Delta$ isométrica a $\psi / \Delta$ (princípio local-global de Pfister);

* $\varphi$ é $G$-subforma de $\psi \Leftrightarrow$ para cada $\Delta$ subgrupo saturado de índice finito de $G \varphi / \Delta$ é $G / \Delta$-subforma de $\psi / \Delta$.

É portanto natural considerar grupos especiais reduzidos $G$ que satisfazem o seguinte príncipio local-global generalizado $([P L G(G)])$ :

Para cada $\phi$ fórmula existencial-positiva (2.4.(e)) com parâmetros em $G$ então :

$G \models \phi[\vec{g}] \quad \Leftrightarrow \quad$ para cada $\Delta$ subgrupo saturado de indice finito de $G \quad G / \Delta \models \phi[\vec{g} / \Delta]$.

Segue deste trabalho que grupos especiais reduzidos que satisfazem tal condição coincidem com os grupos especiais reduzidos que são subgrupos puros de algum grupo especial reduzido profinito que, pela propriedade universal da envoltória profinita, coincidem com os grupos especiais reduzidos cuja imersão canônica em sua envoltória profinita é um $L_{S G}$-monomorfismo puro. (Muito recentemente verificamos que segue deste trabalho que a classe dos grupos especiais reduzidos que satisfazem tal condição é uma classe elementar axiomatizada por sentenças de Horn, um resultado subjacente a $[\mathbf{A T}]$, obtido por processo alternativo.)

Um outro aspecto relevante dos grupos especiais profinitos que colocamos neste momento é que estes são grupos especiais injetivos puros (i.e., grupos especiais que são injetivos quando consideramos a classe dos $L_{S G}$-monomorfismos puros (1.56.(iii))): prova-se em [Ast1] que todo grupo especial finito é injetivo puro; por 2.50 todo grupo especial profinito é um retrato de um produto de grupos especiais finitos e o resultado segue de 1.57 .

Assim o estudo de grupos especiais profinitos nos permitem interpolar questões naturais de difícel encaminhamento. Consideremos as conjecturas abaixo:

(A) Todo grupo especial reduzido pode ser imerso como subgrupo especial puro em algum grupo especial reduzido representável.

(B) Todo grupo especial reduzido satisfaz o princípio local-global generalizado ([PLG]).

(C) RSG possui suficientes injetivos puros reduzidos (1.56.(iv)) .

(D) Injetivos puros reduzidos são retratos de grupos profinitos reduzidos.

A conjectura (A) é uma versão fraca do problema da representação (todo grupo especial reduzido é representável?) mas que é suficientemente forte para implicar que todo grupo especial reduzido satisfaz a conjectura de Marshall e que todo grupo especial satisfaz a conjectura generalizada de Lam (ver [DM3]). Ela delimita a extensão da noção de grupo especial enquanto codificador da teoria algébrica das formas quadráticas sobre corpos.

A conjectura (C) é natural do ramo da álgebra-homológica.

A conjectura (B) interpola (A) e (C):

* (B) implica (A) ;

* A conjunção de (D) e (C) é equivalente a (B).

Acrescentamos que tais conjecturas são legítimas: não são conhecidos contra-exemplos para nenhuma dessas colocações. 
Em termos de perspectivas futuras, a abordagem topológico-analítica dos grupos especiais profinitos sugere, sendo esses instâncias de grupos topológicos "bem comportados" (comutativos e compactos-Hausdorffs), que o estudo e utilização da medida de Haar a eles associada venha a ser um subsídio relevante na compreensão dos $S G$-morfismos entre grupos profinitos e no processo de "obter testemunhas" para tipos constituídos por sentenças existenciais-positivas com parâmetros em grupos especiais profinitos.

As noções e resultados novos, mais importantes e presentes nos Capítulos preparatórios, serão identificados pelo símbolo $\natural$. 


\section{Capítulo 1}

\section{Preliminares}

Neste Capítulo reuniremos, para a conveniência do leitor e referência futura, algumas construções fundamentais em tudo que será discutido aqui.

\subsection{Ordens Parciais, Filtros e Ultrafiltros}

Todo conteúdo desta seção, exceto o Lema 1.6, pode ser encontrado em textos standard de Teoria de Reticulados, como por exemplo [BD].

Definição 1.1. Uma relação binária $\leq e m$ um conjunto I é uma ordem parcial se para todo $i, j, k \in I$

[op 1] : $i \leq i$;

[op 2] : $i \leq j$ ej $\leq i \quad \Rightarrow \quad i=j$;

[op 3] : $i \leq j$ e $j \leq k \Rightarrow i \leq k$.

Indicamos por $\perp$ (ou 0) e T (ou 1) o maior e menor elemento de $I$, se existirem.

Para $i \in I$ definimos $i^{\leftarrow}=\{j \in I: j \leq i\} \quad$ e $\quad i^{\rightarrow}=\{j \in I: i \leq j\}$.

Se uma relação $\leq$ satisfizer apenas [op 1] e [op 3] dizemos que esta é uma pré-ordem parcial.

Exemplo 1.2. Se $\langle I, \leq\rangle$ é um conjunto pré-parcialmente ordenado, indicamos por $\langle I, \leq\rangle^{o p}=$ $\langle I, \geq\rangle$ o oposto (ou dual) de $I$, isto é, o conjunto parcialmente pré-ordenado cujo domínio é $I$ e cuja ordem é dada por $i \geq j$ sse $^{1} j \leq i$.

Definição 1.3. Seja $\langle I, \leq\rangle$ um conjunto parcialmente pré-ordenado.

\footnotetext{
${ }^{1}$ Nossa abreviacao para se e somente se.
} 
(i) $\langle I, \leq\rangle$ é dirigido para cima ${ }^{2}$ se $I \neq \emptyset$ e para todo $i, j \in I, \quad i \rightarrow \cap j \rightarrow \neq \emptyset$.

(ii) $\langle I, \leq\rangle$ é dirigido para baixo ${ }^{3}$ se $I \neq \emptyset$ e para todo $i, j \in I, \quad i^{\leftarrow} \cap j^{\leftarrow} \neq \emptyset$.

Quando fizermos menção a conjuntos pré-ordenados dirigidos sempre estaremos nos referindo a pré-ordens dirigidas para cima.

Está claro que um conjunto ordenado $\langle I, \leq\rangle$ é dirigido para cima sse o conjunto ordenado oposto $\langle I, \leq\rangle^{o p}$ é dirigido para baixo e vice-versa.

Definição 1.4. Seja I um conjunto:

(i) Um subconjunto $S$ de $2^{I}{ }^{4}$ tem a propriedade da interseção finita (pif) se para todo $S^{\prime} \in 2_{\text {fin }}^{S} \doteq\left\{S^{\prime} \in 2^{S}: S^{\prime} \subseteq_{f} S\right\}^{5}$ temos $\bigcap S^{\prime} \neq \emptyset$.

(ii) Um filtro em I é um filtro na álgebra booleana das partes de $I$, ou seja é um subconjunto $F$ de $2^{I}$ tal que para todo $J, K \in 2^{I}$

[fil 0] : $I \in F$;

[fil 1] : $J, K \in F \quad \Rightarrow \quad J \cap K \in F$;

[fil 2] : $J \in F$ e $J \subseteq K \quad \Rightarrow K \in F$.

(iii) O conjunto dos filtros em I, ordenado por inclusão, é um reticulado completo onde ínfimos coincidem com intersȩ̧óes. $\{I\}$ e $2^{I}$ são respectivamente o menor e o maior filtro $\mathrm{em} I$.

(iv) Um filtro $F$ em $I$ é próprio se $F \neq 2^{I}$. Um filtro próprio $F$ em $I$ é um ultrafiltro se for maximal entre os filtros próprios de $I$ na ordem da inclusão em $2^{I}$, isto é, para todo filtro $G$ em I

$$
F \subseteq G \Rightarrow F=G \text { ou } \quad G=2^{I} .
$$

Indicamos por $\mathcal{S}\left(2^{I}\right)$ (ou $U l t(I)$ ) o conjunto dos ultrafiltros em $I$.

(v) $S e\langle I, \leq\rangle$ é um conjunto parcialmente ordenado, dizemos que um filtro $F$ em $I$ é dirigido para cima (respectivamente dirigido para baixo) se

$$
\text { Para todo } i \in I, \quad i^{\rightarrow} \in F\left(\text { respectivamente } i^{\leftarrow} \in F\right) \text {. }
$$

$U l t(I, \leq)^{(\rightarrow)}$ e Ult $(I, \leq)^{(\leftarrow)}$ indicam, respectivamente, o conjunto dos ultrafiltros dirigidos para cima e dirigidos para baixo em $I$.

Algumas das propriedades bem conhecidas de filtros e ultrafiltros estão reunidas na:

Proposição 1.5. Seja I um conjunto, $F$ um filtro em $I$ e $S \subseteq 2^{I}$.

\footnotetext{
${ }^{2}$ Muitos autores usam as expressões filtrante ou filtrante para direita.

${ }^{3}$ Alternativamente cofiltrante ou filtrante para esquerda.

${ }^{4} \mathrm{O}$ conjunto das partes de $I$.

${ }^{5} \subseteq_{f}$ indica subconjunto finito.
} 
(i) F é próprio sse $\emptyset \notin F$.

(ii) Seja $[S]=\left\{J \in 2^{I}:\right.$ Existe $S^{\prime} \subseteq$ S tal que $\left.\bigcap S^{\prime} \subseteq J\right\}$.

$[S]$ sendo o menor filtro em $I$ que contém o conjunto $S$ é denominado o filtro gerado por $\boldsymbol{S}$ em $I$.

Temos que $[S]$ é próprio sse $S$ tem a pif.

(iii) As seguintes condições são equivalentes:

(1) F é um ultrafiltro.

(2) Para todo $J \in 2^{I}, \quad J \in F \quad$ ou $\quad J^{c} \in F .{ }^{6}$

(3) Para todo $J, K \in 2^{I}, J \cup K \in F \Rightarrow J \in F \quad$ ou $K \in F$.

(iv) (Birkhoff-Stone) $S e S \subseteq 2^{I}$ tem a pif então existe um ultrafiltro $G$ em I tal que $S \subseteq G$.

Lema 1.6. Seja $\langle I, \leq\rangle$ um conjunto parcialmente ordenado.

(i) Se $\langle I, \leq\rangle$ é dirigido para cima, então Ult $(I, \leq)^{(\rightarrow)} \neq \emptyset$.

(ii) Se $\langle I, \leq\rangle$ é dirigido para baixo, então $\operatorname{Ult}(I, \leq)^{(\leftarrow)} \neq \emptyset$.

Prova. Verificaremos apenas (ii) posto que o raciocínio para (i) é dual.

Porque $\langle I, \leq\rangle$ é dirigido para baixo verificamos, por indução em $n \in \omega$, que para cada $\left\{i_{0}, \ldots, i_{n-1}\right\} \subseteq I$ existe $j \in I$ tal que $j \leq i_{0}, \ldots i_{n-1}$, logo: $\emptyset \neq j^{\leftarrow} \subseteq \bigcap_{m<n} i_{m}^{\leftarrow}$.

Portanto o conjunto $S=\left\{i^{\leftarrow}: i \in I\right\}$ tem a pif e por 1.5.(iv) existe um ultrafiltro $U$ tal que $S \subseteq \mathrm{U}$.

\subsection{Construções categoriais}

Referências para esta seção são [Mac] e [Mit]. Muitas das construções discutidas abaixo aparecem no Capítulo I de [BD]. Devemos registrar que a leitura de [MP] e [AR] é interessante, tanto do ponto de vista categorial quanto da Lógica e da Teoria dos Modelos.

\subsubsection{Categorias e Morfismos}

Definição 1.7. Uma categoria $\mathcal{A}$ é um $\operatorname{par}\langle\operatorname{Obj}(\mathcal{A}), \mathcal{M}(\mathcal{A})\rangle$ onde $\operatorname{Obj}(\mathcal{A})$ é uma classe cujos elementos são os objetos de $\mathcal{A}$ e $\mathcal{M}(\mathcal{A})$ é uma classe que é a união disjunta

$$
\mathcal{M}(\mathcal{A})=\coprod_{(A, B) \in O b j(\mathcal{A})}[A, B]_{\mathcal{A}}
$$

\footnotetext{
${ }^{6} J^{c}=\mathrm{I}-\mathrm{J}$ é o complemento de $J$ em $I$.
} 
onde $[A, B]_{\mathcal{A}}$ é uma classe (possivelmente vazia), denominado a classe de morfismos de $A$ para $B$ em $\mathcal{A}$. Também é utilizada a notação $\mathcal{A}(A, B) \underset{\text { not }}{=}[A, B]_{\mathcal{A}}$ e quando $\mathcal{A}$ estiver claro no contexto, escrevemos $[A, B]$ no lugar de $[A, B]_{\mathcal{A}}$. Além disso, para cada tripla de objetos de $\mathcal{A}$, $\langle A, B, C\rangle$, temos uma função

$$
[A, B] \times[B, C] \longrightarrow[A, C]:\langle f, g\rangle \mapsto g \circ f,
$$

denominada composição, que verifica as seguintes condiçôes :

[o 1] : Quando estiver definida, a composição é associativa;

[o 2] : Para $A \in \operatorname{Obj}(\mathcal{A})$, existe $I d_{A}{ }^{7}$ em $[A, A]$, tal que para $B \in \operatorname{Obj}(\mathcal{A}), f \in[A, B]$ e $g \in$ $[B, A]$,

$$
f \circ I d_{A}=f \quad \text { e } I d_{A} \circ g=g .
$$

$O$ morfismo $I d_{A}$ é único e chama-se a identidade do objeto $A$. Se $f \in[A, B]$, dizemos que $A$ é o domínio de $f(\operatorname{dom} f=A)$ e que $B$ é o codomínio ${ }^{8}$ de $f($ codom $f=B)$. Utilizaremos notação funcional standard para morfismos. Assim,

$$
f: A \longrightarrow B, A \stackrel{f}{\longrightarrow} B
$$

são sinônimos com $f \in[A, B]$.

Eventualmente abusaremos escrevendo $A \in \mathcal{A}$ para $A \in \operatorname{Obj}(\mathcal{A})$ e $(A \stackrel{f}{\rightarrow} B) \in \mathcal{A}$ para $f \in \mathcal{A}(A, B)$.

Definição 1.8:Seja $\mathcal{A}$ uma categoria.

(i) $\mathcal{A}$ é dita uma categoria localmente pequena se para $A, B \in O b j(\mathcal{A})$ temos que a classe $\mathcal{A}(A, B)$ é imprópria (é um conjunto).

(ii) $\mathcal{A}$ é uma categoria pequena quando as classes $\operatorname{Obj}(\mathcal{A})$ e $\mathcal{M}(\mathcal{A})$ são conjuntos.

Definição 1.9. Sejam $\mathcal{A}, \mathcal{B}$ categorias. $\mathcal{B}$ é uma subcategoria de $\mathcal{A}$ sse

$$
\left\{\begin{array}{c}
\operatorname{Obj}(\mathcal{B}) \subseteq \operatorname{Obj}(\mathcal{A}) \\
\forall A, B \in \operatorname{Obj}(\mathcal{B}), \quad[A, B]_{\mathcal{B}} \subseteq[A, B]_{\mathcal{A}} e \\
\text { Identidades e composições de } \mathcal{B} \text { são restrições das de } \mathcal{A} .
\end{array}\right.
$$

$\mathcal{B}$ é uma subcategoria plena de $\mathcal{A}$ se $[A, B]_{\mathcal{B}}=[A, B]_{\mathcal{A}}$, para todo $A, B$ em $\operatorname{Obj}(\mathcal{B})$.

Exemplo 1.10. Seja $\langle I, \leq\rangle$ um conjunto parcialmente pré-ordenado (1.1).

Há uma categoria naturalmente associada à $I$, também indicada por $I$, construída declarando $\operatorname{Obj}(I)=I$ e $\mathcal{M}(I)=\leq$. Ou seja, para cada $i, j \in I$ :

\footnotetext{
${ }^{7}$ Notação alternativa $1_{A}$.

${ }^{8}$ As vezes, contradomínio.
} 


$$
[i, j]= \begin{cases}\{(i, j)\} & \text { se } i \leq j \\ \emptyset & \text { caso contrário. }\end{cases}
$$

Para cada $i \in I$, o par $(i, i)$ é a identidade de $i$. A composição é a única possível, isto é, se $i \leq j$ $\leq k$, então o único morfismo de $i$ para $j$, composto com o único morfismo de $j$ para $k$, fornece o único morfismo de $j$ para $k$.

Assim, todo conjunto parcialmente pré-ordenado pode ser considerado como uma categoria (pequena).

Em particular, todo ordinal $\alpha$ origina uma categoria $\underline{\alpha}$. Assim:

* 1 é a (essencialmente única) categoria com um único objeto e uma única flecha;

* $\underline{0}$ é a categoria vazia, a única categoria cuja classe de objetos e a classe de flechas são ambas vazias.

Além disso toda categoria $\mathcal{A}$ origina uma "classe parcialmente pré-ordenada" : $(\operatorname{Obj}(\mathcal{A}), \leq)$ onde para cada $A, B \in O b j(\mathcal{A})$ definimos $A \leq B$ sse $\mathcal{A}(A, B) \neq \emptyset$.

Exemplo 1.11. A cada categoria $\mathcal{A}$ corresponde uma categoria dual, indicada por $\mathcal{A}^{o p}$, definida da seguinte forma :

$* \operatorname{Obj}\left(\mathcal{A}^{o p}\right)=\operatorname{Obj}(\mathcal{A})$;

* Para $A, B \in \operatorname{Obj}(\mathcal{A}),[A, B]_{\mathcal{A}^{o p}}=[B, A]_{\mathcal{A}}$;

* Identidades e composições são reversões das identidades e composições originais.

Está claro que $\left(\mathcal{A}^{o p}\right)^{o p}=\mathcal{A}$. Assim, todo resultado em Teoria das Categorias na realidade representa dois : se um fato $S$, de natureza categorial, vale em $\mathcal{A}$, então o seu dual vale em $\mathcal{A}^{o p}$. Além disso, se as hipóteses utilizadas para provar $S$ também valem em $\mathcal{A}^{o p}$, então o seu dual é verdadeiro em $\mathcal{A}=\left(\mathcal{A}^{o p}\right)^{o p}$.

Exemplo 1.12. Sejam $\mathcal{A}_{0}, \mathcal{A}_{1}$ categorias. O produto de $\mathcal{A}_{0}$ e $\mathcal{A}_{1}, \mathcal{A}_{0} \times \mathcal{A}_{1}$, é a categoria definida da seguinte forma :

* $\operatorname{Obj}\left(\mathcal{A}_{0} \times \mathcal{A}_{1}\right)=\operatorname{Obj}\left(\mathcal{A}_{0}\right) \times \operatorname{Obj}\left(\mathcal{A}_{1}\right)$;

* Sejam $A=\left\langle A_{0}, A_{1}\right\rangle$ e $B=\left\langle B_{0}, B_{1}\right\rangle$ objetos em $\mathcal{A}_{0} \times \mathcal{A}_{1}$, então:

$$
\left(\mathcal{A}_{0} \times \mathcal{A}_{1}\right)(A, B)=\mathcal{A}_{0}\left(A_{0}, B_{0}\right) \times \mathcal{A}_{1}\left(A_{1}, B_{1}\right)
$$

* Identidades e composições: "componente a componente".

Está claro que esta definição pode ser generalizada para qualquer conjunto de componentes.

Exemplo 1.13. A cada categoria $\mathcal{A}$ associamos outra categoria $\mathcal{A}^{\bullet \rightarrow \bullet}$, a categoria das flechas de $\mathcal{A}$ : 
* $\operatorname{Obj}\left(\mathcal{A}^{\bullet \rightarrow \bullet}\right)=\mathcal{M}(\mathcal{A})$

* Para cada par de flechas de $\mathcal{A},\left(A_{0} \stackrel{f}{\longrightarrow} A_{1}\right)$ e $\left(A_{0}^{\prime} \stackrel{f^{\prime}}{\longrightarrow} A_{1}^{\prime}\right)$ temos:

$$
\mathcal{A}^{\bullet \rightarrow \bullet}\left(f, f^{\prime}\right)=\left\{\left(h_{0}, h_{1}\right) \in \mathcal{A}\left(A_{0}, A_{0}^{\prime}\right) \times \mathcal{A}\left(A_{1}, A_{1}^{\prime}\right): f^{\prime} \circ h_{0}=h_{1} \circ f\right\}
$$

"Morfismos em categorias de flechas são quadrados comutativos:"

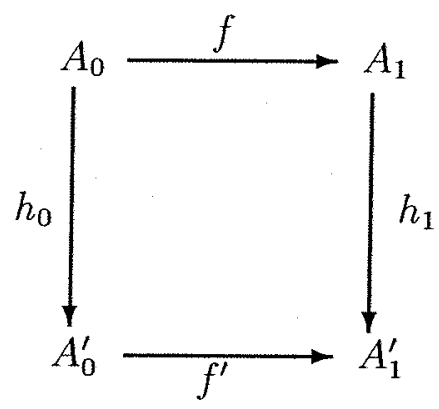

* Identidades e composições: "componente a componente" .

Exemplo 1.14. Sejam $\mathcal{A}$ uma categoria e $A$ um objeto em $\mathcal{A}$. Definimos duas categorias, $\mathcal{A}_{A}$ e $\mathcal{A}^{A}$, como segue :

$\left[\mathcal{A}_{A} 1\right]$ : Os objetos de $\mathcal{A}_{A}$ são morfismos em $\mathcal{A}, f: A \longrightarrow B, \operatorname{com} B \in \operatorname{Obj}(\mathcal{A})$;

$\left[\mathcal{A}_{A} 2\right]:$ Se $I=(A \stackrel{f}{\longrightarrow} B)$ e $J=(A \stackrel{g}{\longrightarrow} C)$ são objetos em $\mathcal{A}_{A}$, um morfismo em $\mathcal{A}_{A}, I \stackrel{h}{\longrightarrow} J$, é um morfismo $h \in[B, C]_{\mathcal{A}}$, tal que $h \circ f=g$.
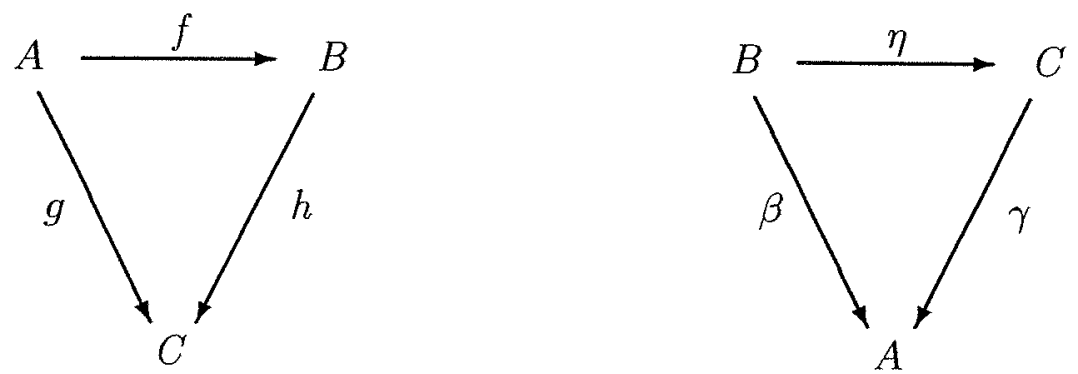

$\left[\mathcal{A}^{A} 1\right]:$ Os objetos de $\mathcal{A}^{A}$ são morfismos em $\mathcal{A}, f: B \longrightarrow A, \operatorname{com} B \in \operatorname{Obj}(\mathcal{A}) ;$

$\left[\mathcal{A}^{A} 2\right]:$ Se $I=(B \stackrel{\beta}{\longrightarrow} A)$ e $J=(C \stackrel{\gamma}{\longrightarrow} A)$ são objetos em $\mathcal{A}^{A}$, um morfismo em $\mathcal{A}^{A}, I \stackrel{\eta}{\longrightarrow} J$, é um morfismo $\eta \in[B, C]_{\mathcal{A}}$, tal que $\gamma \circ \eta=\beta$.

$\mathcal{A}_{A}$ é a categoria das $A$-algebras em $\mathcal{A} ; \mathcal{A}^{A}$ é a categoria dos $\mathcal{A}$-fibrados sobre $A$.

Definição 1.15. Seja $f \in[A, B]$ um morfismo em uma categoria $\mathcal{A}$.

(i) $f$ é um isomorfismo se admitir um inverso, ou seja, se existir $g \in[B, A]$ tal que $f \circ g=$ $I d_{B}$ e $g \circ f=I d_{A}$. 
(ii) $f$ é uma retração se existir $B \stackrel{g}{\longrightarrow} A$, tal que $f \circ g=I d_{B}$.

(iii) $f$ é uma coretração (ou seção) se existir $B \stackrel{g}{\rightarrow} A$ tal que $g \circ f=I d_{A}$. Neste caso dizemos que $A$ é um retrato de $B$.

(iv) $f$ é épico ou um epimorfismo em $\mathcal{A}$ se para quaisquer morfismos $\alpha, \beta$ em $\mathcal{A}$ que verificam dom $\alpha=$ dom $\beta=B$, temos

$$
\alpha \circ f=\beta \circ f \Rightarrow \alpha=\beta \text {. }
$$

(v) $f$ é mônico ou um monomorfismo em $\mathcal{A}$ se para quaisquer morfismos $\alpha, \beta$ em $\mathcal{A}$, verificando codom $\alpha=\operatorname{codom} \beta=A$, temos

$$
f \circ \alpha=f \circ \beta \Rightarrow \alpha=\beta \text {. }
$$

Assim, $f$ é mônico em $\mathcal{A}$ sse $f$ é épico em $\mathcal{A}^{o p}$.

$E$ frequente nos referirmos a um mônico $A \stackrel{f}{\longrightarrow} B$, como um subobjeto de $B$. Uma categoria $e ́$ dita well-powered se para todo objeto $A \mathrm{em} \mathcal{A}$, existe um conjunto $S$ de subobjetos de $A$, tal que todo subobjeto de $A$ em $\mathcal{A}$ é isomorfo, em $\mathcal{A}^{A}$ (veja 1.14), a um elemento de $S$.

Observação 1.16. Em uma categoria $\mathcal{A}$

(i) Toda retração é épica e toda seção é mônica.

(ii) Se $f \circ g$ é mônico, o mesmo é verdade para $g$.

(iii) Se $f \circ g$ é épico, o mesmo é verdade para $f$.

(iv) $\mathrm{O}$ (iso)morfismo inverso de um isomorfismo é único.

(v) Para cada morfismo $f$ são equivalentes:

* $f$ é isomorfismo.

* $f$ é retração e monomorfismo.

* $f$ é seção e epimorfismo.

Observação 1.17. Seja $A \stackrel{f}{\longrightarrow} B$ uma função em Set, a categoria dos conjuntos. Então :

(i) $f$ é mônico sse for injetivo.

(ii) $f$ é épico sse for sobrejetivo.

(iii) O enunciado " $f$ é uma retração sse for sobrejetivo" é equivalente ao Axioma da Escolha.

Definição 1.18. Seja $\mathcal{A}$ uma categoria. Um objeto $A$ em $\mathcal{A}$ é inicial (respec. final) se para todo $B \in \operatorname{Obj}(\mathcal{A}),[A, B]$ (respec. $[B, A]$ ) tem apenas um elemento. Está claro que objetos iniciais $e$ finais são únicos, a menos de isomorfismo. Escrevemos 0 (ou $\perp$ ) e 1 (ou $\mathrm{T})$, respectivamente, para os objetos inicial e final em $\mathcal{A}$, se existirem. 
Exemplo 1.19. Na categoria dos conjuntos, Set, $\emptyset$ é o objeto inicial, enquanto que $\{\emptyset\}$ (ou qualquer outro conjunto unitário) é objeto final.

\subsubsection{Funtores e Transformações Naturais}

Definição 1.20. Sejam $\mathcal{A}, \mathcal{B}$ categorias. Um funtor (covariante), $F: \mathcal{A} \longrightarrow \mathcal{B}$, é uma regra que associa

* A cada objeto $A$ em $\mathcal{A}$, um objeto $F(A)$ em $\mathcal{B}$;

* A cada morfismo $f \in[A, B]_{\mathcal{A}}$, um morfismo $F(f) \in[F(A), F(B)]_{\mathcal{B}}$, tal que

$$
\text { (1) } F\left(I d_{A}\right)=I d_{F(A)} ; \quad \text { (2) } F(f \circ g)=F(f) \circ F(g) \text {, }
$$

onde $\mathcal{A} \in \operatorname{Obj}(\mathcal{A})$ e $f, g$ são morfismos tais que codom $g=\operatorname{dom} f$. Note que para todo $A, B \in \operatorname{Obj}(\mathcal{A}), F$ induz uma função

$$
[A, B]_{\mathcal{A}} \longrightarrow[F(A), F(B)]_{\mathcal{B}}, \quad f \mapsto F(f) .
$$

$F$ é fiel se esta função é injetora e pleno se for sobrejetora.

Um funtor contravariante, $G: \mathcal{A} \longrightarrow \mathcal{B}$, é um funtor de $\mathcal{A}^{\text {op }}$ para $\mathcal{B}$.

Se $F: \mathcal{A} \longrightarrow \mathcal{B}$ e $G: \mathcal{B} \longrightarrow \mathcal{C}$ são funtores, a sua composição é o funtor

$$
G \circ F: \mathcal{A} \longrightarrow \mathcal{C}
$$

dado por

* Se $A \in \operatorname{Obj}(\mathcal{A}),(G \circ F)(A)=G(F(A))$;

* Se $A \stackrel{f}{\longrightarrow} B$ é um morfismo em $\mathcal{A},(G \circ F)(f)=G(F(f))$.

Exemplo 1.21. Se $\mathcal{A}$ é uma categoria, indicamos por $I d_{\mathcal{A}}$ o funtor identidade de $\mathcal{A}$ em $\mathcal{A}$, i.e., o funtor que associa todo objeto e morfismo em $\mathcal{A}$ a eles mesmos.

Exemplo 1.22. Se $\mathcal{C}$ é uma categoria, para cada categoria $\mathcal{I}$ e cada objeto $A \in O b j(\mathcal{C})$ indicamos por $\delta_{\mathcal{I}}(A)$ o funtor constante de valor $A$ e base $\mathcal{I}$, i.e., $\delta_{\mathcal{I}}(A): \mathcal{I} \longrightarrow \mathcal{C}$ é o funtor que associa $A$ a todo objeto de $\mathcal{I}$ e $I d_{A}$ a todo morfismo em $\mathcal{I}$.

Definição 1.23. Uma categoria $\mathcal{A}$ é dita baseada em conjuntos ${ }^{9}$ se existe um funtor fiel de $\mathcal{A}$ para Set .

\footnotetext{
${ }^{9}$ Set-based, em inglês.
} 
Em uma categoria baseada em conjuntos, todo morfismo injetor ${ }^{10}$ é mônico e todo morfismo sobrejetor é épico.

Exemplo 1.24. Se $\mathcal{A}$ e $\mathcal{B}$ são categorias e $\mathcal{A}$ tem estrutura "mais rica" do que $\mathcal{B}$, há um funtor esquecimento de $\mathcal{A}$ para $\mathcal{B}$, que esquece a estrutura mais rica em $\mathcal{A}$. Os exemplos seguintes devem ajudar a esclarecer este conceito :

(0) Categorias baseadas em conjuntos.

(1) Seja $\mathcal{L}$ a categoria dos reticulados e Po a categoria dos conjuntos parcialmente ordenados. Há um funtor esquecimento de $\mathcal{L}$ para $\mathbf{P o}$, que associa a cada reticulado o conjunto parcialmente ordenado subjacente e a cada morfismo de reticulados a mesma função considerada como função crescente.

(2) Há um funtor esquecimento da categoria $\mathcal{D}$ dos reticulados distributivos para a categoria $\mathcal{L}$.

(3) Sejam Ba a categoria das álgebras de Boole e $\mathbf{H a}$ a categoria das álgebras de Heyting. $\mathrm{O}$ funtor esquecimento de $\mathbf{B a}$ para $\mathbf{H a}$, associa a cada álgebra de Boole sua estrutura de álgebra de Heyting subjacente $(x \rightarrow y=\neg x \vee y)$ e a cada morphismo de álgebra de Boole a função correspondente considerada como morfismo de álgebras de Heyting.

Exemplo 1.25. Seja $\mathcal{A}$ uma categoria localmente pequena. Um objeto $A$ em $\mathcal{A}$ induz dois funtores de $\mathcal{A}$ para Set, $h_{A}$ e $h^{A}$, definidos da seguinte forma:

$\left[h_{A} 1\right]$ : Para cada $B \in \operatorname{Obj}(\mathcal{A}), h_{A}(B)=[A, B]$, o conjunto dos morfismos de $A$ para $B$ em $\mathcal{A}$. $\left[h_{A} 2\right]:$ Para um morfismo $B \stackrel{g}{\longrightarrow} C$ em $\mathcal{A}, h_{A}(g):[A, B] \longrightarrow[A, C]$ é dado por $f \mapsto g \circ f$.
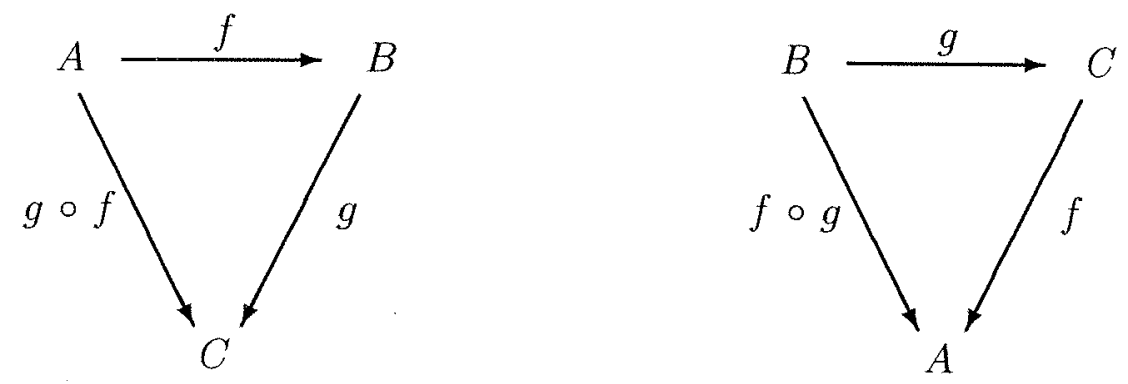

$\left[h^{A} 1\right]:$ Para cada $B \in \operatorname{Obj}(\mathcal{A}), h^{A}(B)=[B, A]$, o conjunto dos morfismos de $B$ para $A$ em $\mathcal{A}$. $\left[h_{A} 2\right]:$ Para um morfismo $B \stackrel{g}{\rightarrow} C \mathrm{em} \mathcal{A}, h^{A}(g):[C, A] \longrightarrow[B, A]$ é dado por $f \mapsto f \circ g$.

Note que $h_{A}$ é covariante, enquanto que $h^{A}$ é contravariante. Estes funtores denominam-se funtores de morfismo de parâmetro $A$.

\footnotetext{
${ }^{10}$ Isto é, um morfismo da categoria original que é transformado pelo funtor considerado numa aplicação injetora.
} 
Observação 1.26. Sejam $\mathcal{A}, \mathcal{B}$ e $\mathcal{C}$ categorias. Um funtor de $\mathcal{A} \times \mathcal{B}$ para $\mathcal{C}$ é usualmente chamado de bifuntor, que pode ter variâncias distintas em cada coordenada. Por exemplo, pode ser covariante na primeira e contravariante na segunda coordenada.

Note que se $F: \mathcal{A} \longrightarrow \mathcal{B}$ e $G: \mathcal{C} \longrightarrow \mathcal{D}$ são funtores, temos um bifuntor

$$
T \doteq F \times G: \mathcal{A} \times \mathcal{B} \longrightarrow \mathcal{C} \times \mathcal{D}
$$

dado em objetos por $T(A, B)=\langle F(A), G(B)\rangle$ e em morfismos por $T(f, g)=\langle F(f), G(g)\rangle$. Está claro que estas observações podem ser estendidas a qualquer número de componentes.

Exemplo 1.27. Sejam $\mathcal{A}$ e $\mathcal{B}$ categorias localmente pequenas e $F: \mathcal{A} \rightarrow \mathcal{B}$ e $G: \mathcal{B} \rightarrow \mathcal{A}$ funtores de mesma variância. Assumiremos, sem perda de generalidade, que são ambos covariantes. Estes funtores dão origem a bifuntores

$$
{ }_{F} h, h_{G}: \mathcal{A}^{o p} \times \mathcal{B} \longrightarrow \text { Set }
$$

definidos por :

* Para $A \in O b j(\mathcal{A})$ e $B \in O b j(\mathcal{B})$,

$$
\left\{\begin{aligned}
F h(A, B) & =[F(A), B]_{\mathcal{B}} \\
h_{G}(A, B) & =[A, G(B)]_{\mathcal{A}} .
\end{aligned}\right.
$$

* Para um morfismo $\langle f, g\rangle:\langle A, B\rangle \longrightarrow\langle C, D\rangle$ in $\mathcal{A}^{o p} \times \mathcal{B}$,

$$
\left\{\begin{aligned}
F_{F} h(f, g):[F(A), B] & \rightarrow[F(C), D] \text { dado por } l \mapsto g \circ l \circ F(f) . \\
h_{G}(f, g):[A, G(B)] \rightarrow[C, G(D)] \text { dado por } r & \mapsto G(g) \circ r \circ f
\end{aligned}\right.
$$
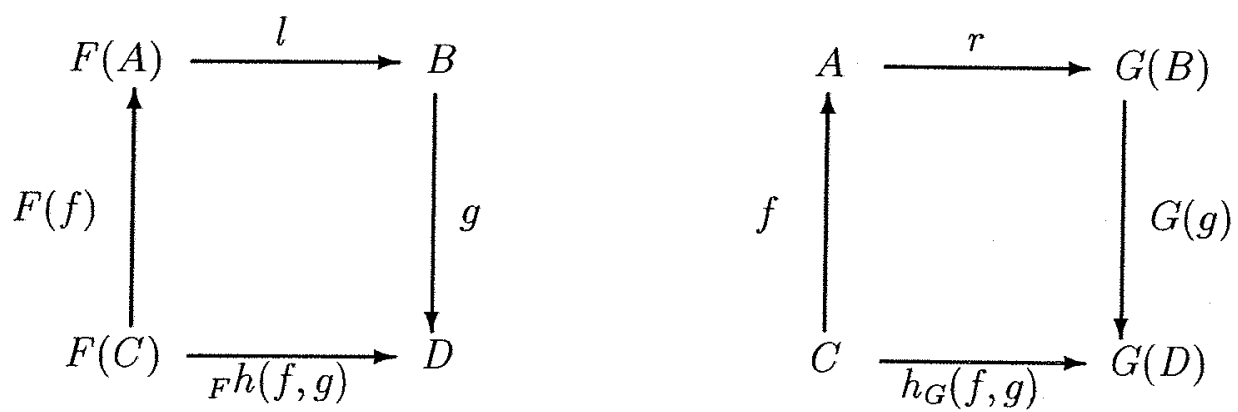

Voltaremos a este Exemplo quando discutirmos a noção de adjunção.

Morfismos entre funtores são denominados transformações naturais :

Definição 1.28. Sejam $\mathcal{A}$ e $\mathcal{B}$ categorias. Consideremos $F, G: \mathcal{A} \longrightarrow \mathcal{B}$ funtores de mesma variância. Uma transformação natural, $\eta: F \longrightarrow G$, é uma aplicação $\eta: \operatorname{Obj}(\mathcal{A}) \longrightarrow \operatorname{Mor}(\mathcal{B})$ tal que:

* Para cada $A \in \operatorname{Obj}(\mathcal{A})$ temos $\eta_{A} \in[F(A), G(A)]_{\mathcal{B}}$ 
* Para todo morfismo $A \stackrel{f}{\longrightarrow} B$ em $\mathcal{A}$, o seguinte diagrama é comutativo :
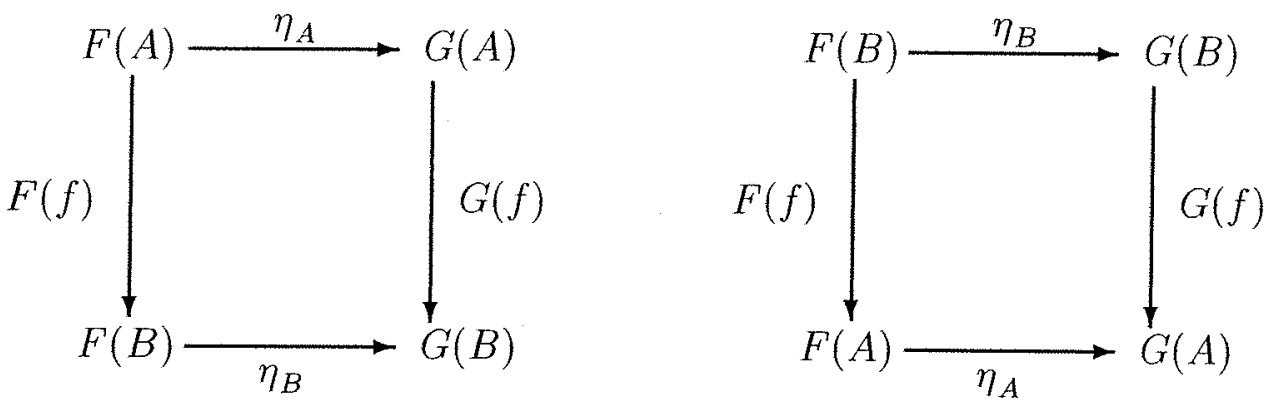

onde o diagrama acima à direita corresponde ao caso em que $F$ e $G$ são contravariantes. No caso em que $\eta_{A}$ é um isomorfismo para todo $A \in O b j(\mathcal{A})$, dizemos que $\eta$ é uma equivalência natural.

Indicaremos por $[F, G]$ ou $N a t(F, G)$ o "conglomerado" 11 das transformações naturais de $F$ para $G$.

Exemplo 1.29. Se $\mathcal{C}$ é uma categoria, para cada categoria $\mathcal{I}$ e cada flecha $f \in \mathcal{C}\left(A, A^{\prime}\right)$ em $\mathcal{C}$ indicamos por $\delta_{\mathcal{I}}(f)$ a transformação natural constante de valor $f$ e base $\mathcal{I}$, i.e., $\delta_{\mathcal{I}}(f) \in \operatorname{Nat}\left(\delta_{\mathcal{I}}(A), \delta_{\mathcal{I}}\left(A^{\prime}\right)\right){ }^{12}$ é a transformação que associa $f$ a todo objeto de $\mathcal{I}$.

\section{Observação 1.30.}

(i) A noção de transformação natural é funtorial em ambos os lados, isto é, para cada $\mathcal{A}$ e $\mathcal{B}$ categorias, $F, G: \mathcal{A} \longrightarrow \mathcal{B}$ funtores de mesma variância e $\eta: F \longrightarrow G$ transformação natural, temos:

* Se $L: \mathcal{A}^{\prime} \longrightarrow \mathcal{A}$ é um funtor então $\eta L: F \circ L \longrightarrow G \circ L$ é uma transformação natural:

$$
\eta L=\left(\eta_{L\left(A^{\prime}\right)}: A^{\prime} \in \operatorname{Obj}\left(\mathcal{A}^{\prime}\right)\right) \text {. }
$$

* Se $R: \mathcal{B} \longrightarrow \mathcal{B}^{\prime}$ é um funtor então $R \eta: R \circ F \longrightarrow R \circ G$ é uma transformação natural:

$$
R \eta=\left(R\left(\eta_{A}\right): A \in O b j(\mathcal{A})\right) \text {. }
$$

(ii) Transformações naturais podem ser compostas: ${ }^{13}$

Dados $F, G, H: \mathcal{A} \longrightarrow \mathcal{B}$ funtores e $F \stackrel{\eta}{\longrightarrow} G, G \stackrel{\mu}{\longrightarrow} H$ transformações naturais, então $\mu \circ \eta: F \longrightarrow H$, dada por

$$
\mu \circ \eta=\left(\mu_{A} \circ \eta_{A}\right)_{A \in O b j(\mathcal{A})}
$$

é a composição (vertical) de $\eta$ e $\mu$.

\footnotetext{
${ }^{11}$ Uma fundamentação conjuntística adequada para as definições e construções categoriais é apresentada em [GV] .

${ }^{12}$ Ver exemplo 1.22 .

${ }^{13}$ Ver seções 4 e 5 do Capitulo 2 em [Mac].
} 
Definição 1.31. Seja $\mathcal{A}$ uma categoria localmente pequena. Um funtor covariante, $F$ : $\mathcal{A} \longrightarrow$ Set é representável se existir $A \in \operatorname{Obj}(\mathcal{A})$ tal que $F$ é naturalmente equivalente $a$ $h_{A}$.

Um resultado fundamental acerca de funtores representáveis é o:

Teorema 1.32. (O Lema de Yoneda) Sejam $\mathcal{A}$ uma categoria localmente pequena e $F: \mathcal{A} \longrightarrow$ Set um funtor. Então :

(i) Para cada objeto $A$ em $\mathcal{A}$, existe uma bijeção

$$
\theta_{A, F}:\left[h_{A}, F\right] \longrightarrow F(A), \theta_{A, F}(\eta)=\eta_{A}\left(I d_{A}\right),
$$

que é natural em $F$ e $A$.

(ii) $\operatorname{Se} A$ e $B$ são objetos em $\mathcal{A}$, a aplicação

$$
\gamma_{A, B}:\left[h_{A}, h_{B}\right] \longrightarrow[B, A], \gamma_{A, B}(\eta)=\theta_{A, h_{B}}(\eta)=\eta_{A}\left(I d_{A}\right),
$$

é uma bijeção, que é natural em $A$ e $B$.

Prova. Para uma prova, veja Lema na pag. 61 de [Mac] ou Lema 2.1 e Corolário 2.2, pag. 97, em [Mit].

\subsubsection{Funtores Adjuntos e Equivalência de Categorias}

Definição 1.33. Com a notação de 1.27, dois funtores $\mathcal{A} \stackrel{F}{\rightarrow} \mathcal{B}$ e $\mathcal{B} \stackrel{G}{\longrightarrow} \mathcal{A}$ são um par adjunto, $(F, G)$, se existir uma equivalência natural de bifuntores, $\rho:{ }_{F} h \longrightarrow h_{G}$. Assim, para cada objeto $\langle A, B\rangle \in \operatorname{Obj}\left(\mathcal{A}^{o p} \times \mathcal{B}\right)$, existe uma bijeção natural

$$
\rho_{A, B}:[F(A), B]_{\mathcal{B}} \longrightarrow[A, G(B)]_{\mathcal{A}}
$$

Dizemos que $F$ é adjunto à esquerda de $G$ e que $G$ é adjunto à direita de $F$.

Observação 1.34. Se $\mathcal{A}$ e $\mathcal{B}$ são categorias provenientes de conjuntos pré-ordenados (ver 1.10) e $\mathcal{A} \stackrel{F}{\longrightarrow} \mathcal{B}$ e $\mathcal{B} \stackrel{G}{\longrightarrow} \mathcal{A}$ são funtores covariantes (funções crescentes) então $(F, G)$ é um par adjunto sse para cada $(A, B) \in \operatorname{Obj}\left(\mathcal{A}^{o p} \times \mathcal{B}\right)$ temos:

$$
F(A) \leq B \quad \Leftrightarrow \quad A \leq G(B) .
$$

Neste caso o par adjunto é denominado uma conexão de Galois covariante.

A noção de conexão de Galois contrariante entre funções decrescentes (funtores contravariantes) é definida dualmente. 
Mais tarde enunciaremos o Teorema do Funtor Adjunto, mas o resultado que segue, uma generalização da construção de objetos livres em categorias de álgebras, é bastante útil.

Teorema 1.35. Se $\mathcal{B} \stackrel{G}{\longrightarrow} \mathcal{A}$ é um funtor covariante, as seguintes condições são equivalentes :

(1) G tem adjunto à esquerda;

(2) Para todo $A \in \operatorname{Obj}(\mathcal{A})$, existe $F(A) \in \operatorname{Obj}(\mathcal{B})$ e um morfismo $\eta_{A}: A \longrightarrow G(F(A))$ em $\mathcal{A}$, verificando a seguinte propriedade universal:

Para todo $B \in O b j(\mathcal{B})$ e todo morfismo $A \stackrel{f}{\longrightarrow} G(B)$ em $\mathcal{A}$, existe um único morfismo $\tilde{f}: F(A) \longrightarrow B$ em $\mathcal{B}$, tal que o diagrama abaixo é comutativo :

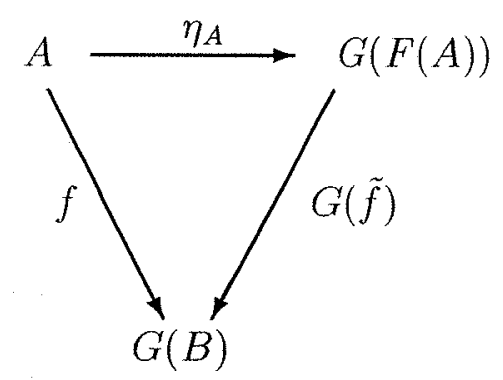
direita.

Evidentemente, o Teorema 1.35 tem um dual, que caracteriza a existência de adjuntos à

Definição 1.36. Seja $\mathcal{A}$ uma subcategoria de $\mathcal{B}$. $\mathcal{A}$ é uma subcategoria refletora de $\mathcal{B}$ se a imersão natural $\mathcal{A} \longrightarrow \mathcal{B}$ tem adjunto à esquerda, denominado funtor de reflexão de $\mathcal{B}$ para $\mathcal{A}$. $\mathcal{A}$ é uma subcategoria corefletora de $\mathcal{B}$ se a imersão natural $\mathcal{A} \longrightarrow \mathcal{B}$ tem adjunto à direita, denominado coreflexão de $\mathcal{B}$ em $\mathcal{A}$.

\section{Exemplo 1.37.}

(i) As seguintes são subcategorias plenas e refletoras de Top, a categoria dos espaços topológicos e funções contínuas:

* A categoria dos espaços $T_{i}, i=1,2,3$;

* A categoria dos espaços completamente regulares;

* A categoria dos espaços booleanos. ${ }^{14}$

(ii) A subcategoria dos espaços topológicos normais é refletora dentro da categoria dos espaços topológicos Hausdorffs.

\footnotetext{
${ }^{14}$ Um espaço topológico é booleano sse for compacto, Hausdorff e possuir uma base constituída por conjuntos clopens (i.e. simultaneamente abertos e fechados) .
} 
(iii) A subcategoria plena dos grupos Abelianos livres de torsão é refletora da categoria Ab dos grupos Abelianos.

(iv) A subcategoria plena $\mathrm{Ab}$ é refletora da categoria dos grupos em geral.

(v) A subcategoria plena dos grupos de torsão é corefletora em Ab.

Definição 1.38. As categorias $\mathcal{A}$ e $\mathcal{B}$ são equivalentes se existirem funtores $F: \mathcal{A} \longrightarrow \mathcal{B} e$ $G: \mathcal{B} \longrightarrow \mathcal{A}$, juntamente com equivalências naturais

$$
\eta: I d_{\mathcal{A}} \longrightarrow(G \circ F) \text { e } \mu:(F \circ G) \longrightarrow I d_{\mathcal{B}} .
$$

$O$ par $(F, G)$ denomina-se uma equivalência entre $\mathcal{A}$ e $\mathcal{B}$. Uma equivalência contravariante denomina-se uma dualidade.

\subsubsection{Diagramas e Limites}

Definição 1.39. Sejam $\mathcal{C}$ uma categoria e $\mathcal{I}$ uma categoria ${ }^{15}$. Um $\mathcal{I}$-diagrama em $\mathcal{C}$ é um funtor $D: \mathcal{I} \longrightarrow \mathcal{C}$.

Observação 1.40. A noção de diagrama é funtorial em ambos os lados, isto é, para cada $D: \mathcal{I} \longrightarrow \mathcal{C}$ um $\mathcal{I}$-diagrama em $\mathcal{C}$, temos:

* Se $T: \mathcal{I}^{\prime} \longrightarrow \mathcal{I}$ é um funtor então $D \circ T: \mathcal{I}^{\prime} \longrightarrow \mathcal{C}$ é um $\mathcal{I}^{\prime}$-diagrama em $\mathcal{C}$.

* Se $F: \mathcal{C} \longrightarrow \mathcal{C}^{\prime}$ é um funtor então $F \circ D: \mathcal{I} \longrightarrow \mathcal{C}^{\prime}$ é um $\mathcal{I}$-diagrama em $\mathcal{C}^{\prime}$.

Exemplo 1.41. Seja $\mathcal{C}$ uma categoria.

(0) Seja $\mathcal{I}=\underline{0}$ a categoria vazia (conforme 1.10) então existe um único $\mathcal{I}$-diagrama em $\mathcal{C}$.

(1) Seja $\mathcal{I}$ a categoria, essencialmente única, com um único objeto $x$ e tal que $[x, x]_{\mathcal{I}}$ tem precisamente dois elementos. Um $\mathcal{I}$-diagrama em $\mathcal{C}$ consiste de um par de flechas paralelas em $\mathcal{C}$,

$$
A \underset{I d_{A}}{\stackrel{e}{\longrightarrow}} A
$$

$\operatorname{com} A \in O b j(\mathcal{C}) ; I d_{A}, e \in \mathcal{C}(A, A)$ e onde : $\left(A \stackrel{I d_{A}}{\longrightarrow} A\right)$ é a identidade de $A$ e $(A \stackrel{e}{\longrightarrow} A)$ é algum endomorfismo de $A$ que é idempotente $(\epsilon \circ e=e)$.

\footnotetext{
${ }^{15}$ Geralmente $\mathcal{I}$ é tomada uma categoria pequena .
} 
(2) Seja $\mathcal{I}$ a categoria, essencialmente única, com dois objetos distintos, $L$ e $R$, e tal que $[L, R]_{\mathcal{I}}$ tem precisamente dois elementos. Um $\mathcal{I}$-diagrama em $\mathcal{C}$ consiste de um par de flechas paralelas em $\mathcal{C}$,

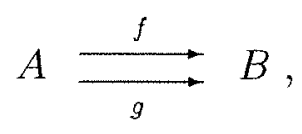

$\operatorname{com} A, B \in \operatorname{Obj}(\mathcal{C})$ e $f, g \in[A, B]$.

(2) Seja $\mathcal{I}$ a categoria cujos objetos são um conjunto $I$ e cuja classe de morfismos contém apenas os morfismos identidade de cada objeto ${ }^{16}$. Um $\mathcal{I}$-diagrama em $\mathcal{C}$ é simplesmente uma família de objetos de $\mathcal{C}$, indexados por $I$.

(3) Seja $\langle I, \leq\rangle$ um conjunto parcialmente pré-ordenado, considerado como uma categoria como no Exemplo 1.10.

(I) Um $I$-diagrama em $\mathcal{A}$ é uma família de objetos, $\left\{A_{i}: i \in I\right\}$, juntamente com morfismos $f_{i j} \in\left[A_{i}, A_{j}\right]$, para $i \leq j$, tal que para todo $i, j, k \in I$

$$
* f_{i i}=I d_{A_{i}}
$$

* Se $i \leq j \leq k$ em $I$, o seguinte diagrama é comutativo :

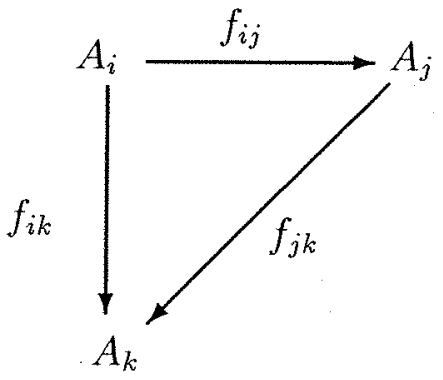

(II) Um $I^{o p}$-diagrama em $\mathcal{A}$ é uma família de objetos $\left\{A_{i}: i \in I\right\}$, juntamente com morfismos $f_{j i} \in\left[A_{j}, A_{i}\right]$, para $i \leq j$, tal que para todo $i, j, k \in I$

$$
* f_{i i}=I d_{A_{i}}
$$

* Se $i \leq j \leq k$ em $I$, o seguinte diagrama é comutativo:

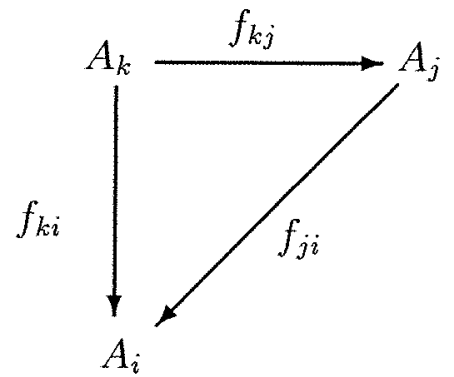

Se $I$ é dirigido (1.3), um $I$-diagrama denomina-se um sistema filtrante em $\mathcal{A}$, enquanto que um $I^{o p}$-diagrama é conhecido como um sistema cofiltrante em $\mathcal{A}$.

Como casos especiais de (4).(I) e (4).(II) temos :

(a) Suponha que $I=\{i, j, k\}$ seja um conjunto ordenado com precisamente três elementos,

\footnotetext{
${ }^{16} \mathcal{I}$ é dita a categoria discreta sobre o conjunto $I$.
} 
tal que $i \leq j, i \leq k$, enquanto que $j$ e $k$ não estão relacionados. Os seguintes diagramas são exemplos de $I$-diagrama e $I^{o p}$-diagrama em $\mathcal{A}$, respectivamente :
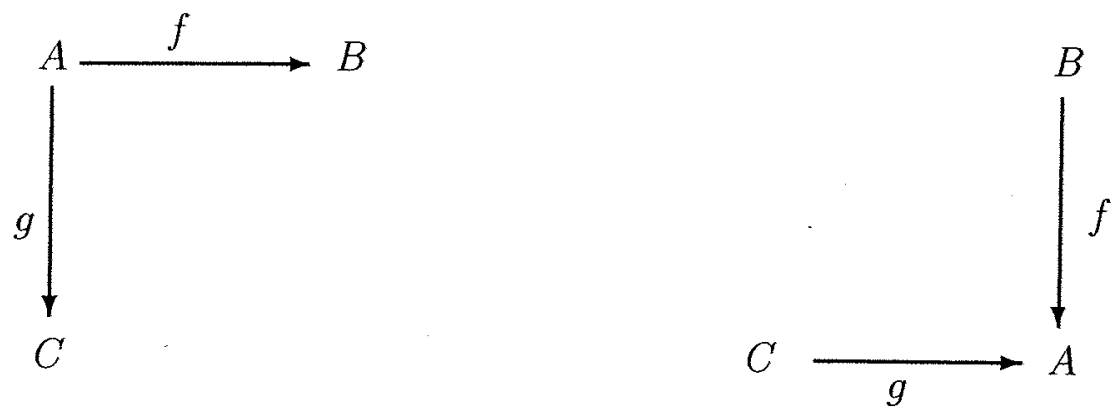

(b) Suponha que $I$ é a álgebra de Boole com quatro elementos, $\{\perp, a, \neg a, T\}$, considerada como uma categoria. Um $I$-diagrama em $\mathcal{A}$ é um quadrado covariante comutativo em $\mathcal{A}$, i.e., um diagrama da forma abaixo à direita $\operatorname{com} k \circ f=g \circ h$.
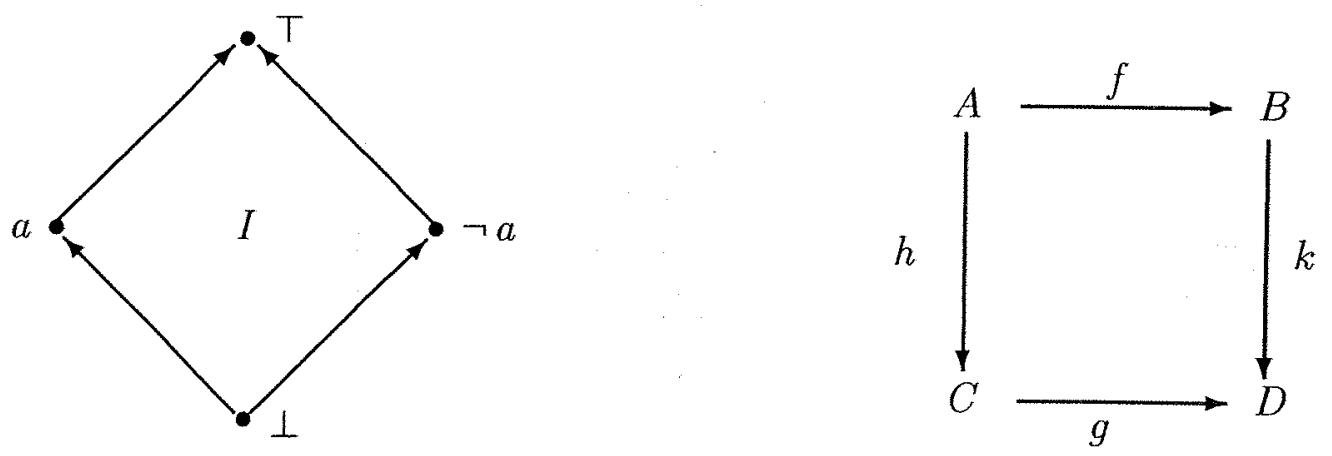

Definição 1.42. Seja $D: \mathcal{I} \longrightarrow \mathcal{C}$ um $\mathcal{I}$-diagrama em $\mathcal{C}$.

(i) Um cone (ou cone projetivo) sobre $D$ é uma transformação natural de algum funtor constante com base $\mathcal{I}$ e a valores em $\mathcal{C}$ para o funtor $F, \pi: \delta_{\mathcal{I}}(A) \rightarrow F$. Portanto um cone consiste de um objeto $A$ em $\mathcal{C}$ (dito o vértice do cone), juntamente com uma sequência de morfismos em $\mathcal{C}$ (dita a família geratriz do cone), $\left(A \stackrel{\pi_{i}}{\longrightarrow} D(i): i \in O b j(\mathcal{I})\right)$ tal que, para cada $(i \stackrel{f}{\rightarrow} j)$ morfismo em $\mathcal{I}$, o diagrama abaixo é comutativo :

$A$

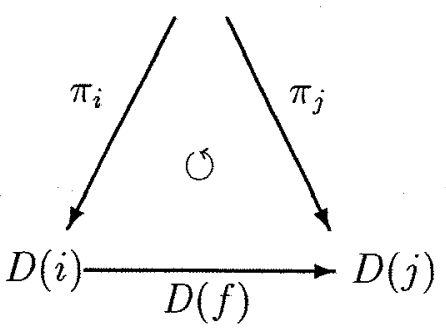

Para cada $(i \stackrel{f}{\rightarrow} j)$ em $\mathcal{I}$.

(ii) $S e \pi=\left(A \stackrel{\pi_{i}}{\rightarrow} D(i): i \in O b j(\mathcal{I})\right)$ e $\pi^{\prime}=\left(A^{\prime} \stackrel{\pi_{\prime}^{\prime}}{\rightarrow} D(i): i \in O b j(\mathcal{I})\right)$ são cones projetivos sobre $D$, um morfismo de cones, $h: \pi \longrightarrow \pi^{\prime}$, é uma transformação natural constante $\delta_{\mathcal{I}}(h)$ : 
$\delta_{\mathcal{I}}(A) \longrightarrow \delta_{\mathcal{I}}\left(A^{\prime}\right)$ tal que $\pi=\pi^{\prime} \circ \delta_{\mathcal{I}}(h)$. Ou seja, um morfismo de cones $h: \pi \longrightarrow \pi^{\prime}$ é constituído de um $\mathcal{C}$-morfismo $h: A \longrightarrow A^{\prime}$ tal que, para todo $j \in O b j(\mathcal{I})$, o diagrama abaixo é comutativo.

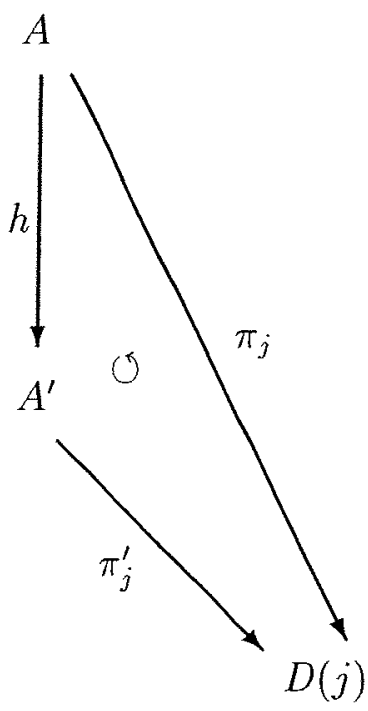

Para cada jem $\mathcal{I}$.

É imediato que a identidade e a composição de morfismos de cones são morfismos de cones e portanto, para cada diagrama $(\mathcal{I} \stackrel{D}{\rightarrow} \mathcal{C})$ temos uma categoria, $\operatorname{Cone}(\mathcal{I} \stackrel{D}{\rightarrow} \mathcal{C})$, dos cones sobre o diagrama $D$.

A noção dual de cone é a de:

Um cocone (ou cone indutivo) sobre um diagrama $D$ em $\mathcal{C}$ é um um objeto $A$ em $\mathcal{C}$ juntamente com uma família de morfismos em $\mathcal{C},\left(D(i) \stackrel{\nu_{i}}{\longrightarrow} A: i \in \operatorname{Obj}(\mathcal{I})\right)$ tal que, para cada $(i \stackrel{f}{\rightarrow} j$ ) morfismo em $\mathcal{I}$, o diagrama abaixo é comutativo :

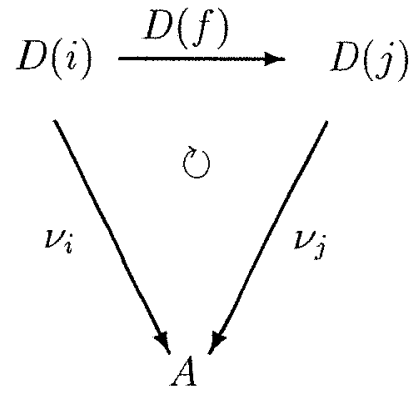

Para cada $(i \stackrel{f}{\rightarrow} j)$ em $\mathcal{I}$.

Por dualidade com o caso de cones, definimos morfismos de co-cones sobre o diagrama $(\mathcal{I} \stackrel{D}{\longrightarrow} \mathcal{C})$, obtendo uma categoria, $\operatorname{coCone}(\mathcal{I} \stackrel{D}{\rightarrow} \mathcal{C})$, com propriedades análogas.

Observação 1.43. 
(i) A noção de cone sobre um diagrama é funtorial em ambos os lados, isto é, se $\pi$ é um cone sobre o diagrama $F: \mathcal{I} \longrightarrow \mathcal{C}$ e $T: \mathcal{I}^{\prime} \longrightarrow \mathcal{I}, R: \mathcal{C} \longrightarrow \mathcal{C}^{\prime}$ são funtores, obtemos funtores:

* $R^{\star}: \operatorname{Cone}(\mathcal{I} \stackrel{D}{\rightarrow} \mathcal{C}) \longrightarrow \operatorname{Cone}\left(\mathcal{I}^{R \circ D} \rightarrow \mathcal{C}^{\prime}\right) \quad * T_{\star}: \operatorname{Cone}(\mathcal{I} \stackrel{D}{\rightarrow} \mathcal{C}) \longrightarrow \operatorname{Cone}\left(\mathcal{I}^{\prime} \stackrel{D \circ T}{\rightarrow} \mathcal{C}\right)$.

(ii) Sejam $D, E: \mathcal{I} \longrightarrow \mathcal{C}$ diagramas e $\tau: D \longrightarrow E$ transformação natural. Então obtemos o funtor:

$$
\tau_{\star}: \operatorname{Cone}(\mathcal{I} \stackrel{D}{\rightarrow} \mathcal{C}) \longrightarrow \operatorname{Cone}(\mathcal{I} \stackrel{E}{\rightarrow} \mathcal{C})
$$
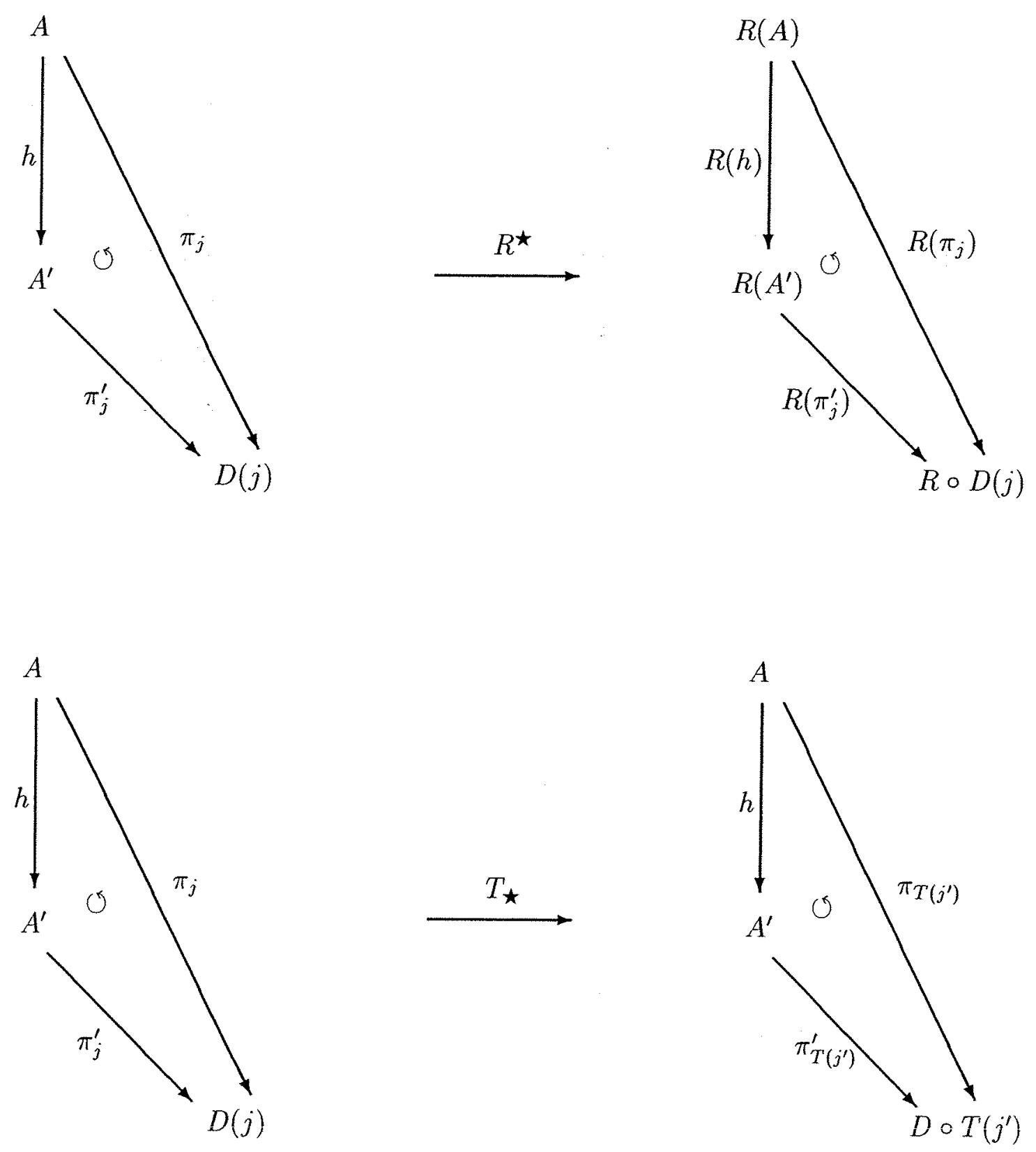

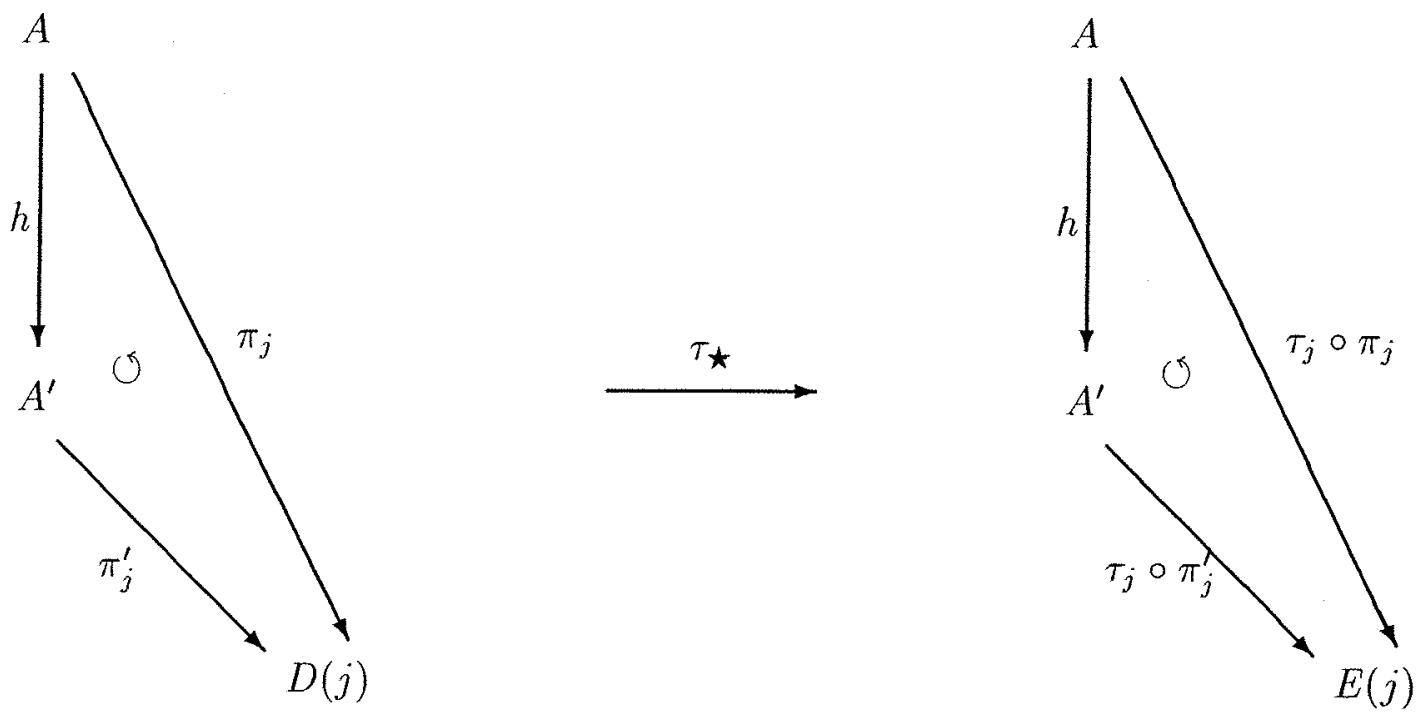

Definição 1.44. Seja $D: \mathcal{I} \longrightarrow \mathcal{C}$ um diagrama em uma categoria $\mathcal{C}$.

$U m$ limite (ou limite projetivo) para $D$ em $\mathcal{C}$ é um objeto final na categoria Cone $(\mathcal{I} \rightarrow \mathcal{C})$; indicamos por lim $D$ o limite de $D$ em $\mathcal{C}$. Dualmente, um colimite (ou limite indutivo) para $D$ em $\mathcal{C}$ é um objeto inicial na categoria coCone $(\mathcal{I} \stackrel{D}{\rightarrow} \mathcal{C})$; indicamos por lim $D$ o colimite de $D$ em $\mathcal{C}$.

Está claro que limites e colimites são únicos, a menos de isomorfismo.

Se $D$ tem limite em $\mathcal{A}$, é usual escrevermos

$$
\stackrel{\lim }{\leftarrow} D=\left(A ;\left\{\pi_{i}: i \in \operatorname{Obj}(\mathcal{I})\right\}\right) \quad \text { ou } \quad A \cong \overleftarrow{i \in I}_{\lim } D(i)
$$

onde $A \in O b j(\mathcal{A})$ é o vértice do cone $\lim _{\longleftarrow} D$ e $\left(A \stackrel{\pi_{i}}{\longrightarrow} D(i)\right)_{i \in O b j(\mathcal{I})}$ são as geratrizes do cone. Analogamente para colimites.

Um funtor $F: \mathcal{A} \longrightarrow B$ preserva limites se para todo $\mathcal{I}$-diagrama $D$ em $\mathcal{A}$, se lim $D$ existe em $\mathcal{A}$, então $\lim (F \circ D)$ existe em $\mathcal{B}$ e o único morfismo de cones $F^{\star}(\lim D) \longleftrightarrow \lim (F \circ D)$ 17 é um isomorfismo.

Notação: $\quad F\left(\lim _{i \in I}^{\longleftarrow} D(i)\right) \stackrel{\cong}{\longleftarrow} \lim (F \circ D)(i)$.

Analogamente, definimos a preservação de colimites por um funtor.

Exemplo 1.45. Seja $\mathcal{C}$ uma categoria.

\footnotetext{
${ }^{17} \operatorname{Ver} 1.43 .(\mathrm{i})$
} 
(i) Se $\mathcal{I}$ é uma categoria como no item (2) de 1.41, um limite para um $\mathcal{I}$-diagrama em uma categoria $\mathcal{C}$ é o ${ }^{18}$ equalizador do par

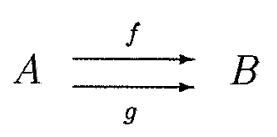

Um colimite para este diagrama é o coequalizador do par $(f, g) \mathrm{em} \mathcal{C}$.

(ii) Se $\mathcal{I}$ é a categoria associada a um conjunto ordenado munido da ordem discreta (i.e. $x \leq y$ sse $x=y$ ) como em 1.41.(3), o limite para um $\mathcal{I}$-diagrama em $\mathcal{C}$ denomina-se o produto da família $\left\{A_{i}: i \in I\right\}$ em $\mathcal{C}$, enquanto que o colimite para este diagrama denomina-se o coproduto desta família em $\mathcal{C}$.

Em particular, se $\mathcal{I}=\underline{0}$ é a categoria vazia, então o limite ou produto do diagrama vazio (ver item $(0)$ de 1.41) é o objeto final em $\mathcal{C}$; o colimite ou coproduto do diagrama vazio é o objeto inicial em $\mathcal{C}$.

(iii) Seja I um conjunto parcialmente ordenado e dirigido.

(1) $\mathrm{O}$ colimite de um $I$-diagrama em $\mathcal{C}$ é denominado limite indutivo filtrante do sistema filtrante de objetos e morfismos determinado por este $I$-diagrama.

(2) O limite de um $I^{o p}$-diagrama em $\mathcal{C}$ é denominado limite projetivo cofiltrante do sistema cofiltrante de objetos e morfismos determinado pelo $I^{o p}$-diagrama.

(iv) Se $I$ é o conjunto parcialmente ordenado do item 3.(a) em 1.41, o limite de um $I$-diagrama em $\mathcal{C}$ é o pushout dos seus morfismos. O colimite de um $I^{o p}$-diagram em $\mathcal{C}$ é o pullback ou produto fibrado dos seus morfismos.

(v) Seja $\mathcal{I}$ uma categoria como no item (1) de 1.41.

Seja $A \underset{I d_{A}}{\stackrel{e}{\rightrightarrows}} A$ um $\mathcal{I}$-diagrama em $\mathcal{C}$.

(1) Existem bijeções naturais entre os conjuntos constituídos:

* Por cisões do idempotente e, i.e., por elementos $\left(A^{\prime}, p, s\right)$ onde $A^{\prime} \in \mathcal{C}$ e $A \underset{s}{\stackrel{p}{\rightleftarrows}} A^{\prime}$ é diagrama em $\mathcal{C}$ tal que $s \circ p=e: A \rightarrow A, p \circ s=I d_{A^{\prime}}: A^{\prime} \rightarrow A^{\prime}$.

* Por elementos $\left(A^{\prime}, p\right)$ onde $A^{\prime} \in \mathcal{C}$ e $A \stackrel{p}{\rightarrow} A^{\prime}$ é coequalizador do diagrama $A \underset{I d_{A}}{\stackrel{e}{\rightrightarrows}} A$.

* Por elementos $\left(A^{\prime}, s\right)$ onde $A^{\prime} \in \mathcal{C}$ e $A^{\prime} \stackrel{s}{\rightarrow} A$ é equalizador do diagrama $A \underset{I d_{A}}{\stackrel{e}{\rightrightarrows}} A$.

(2) Se $\mathcal{C}$ é categoria que possui limites indutivos filtrantes temos que os conjuntos acima

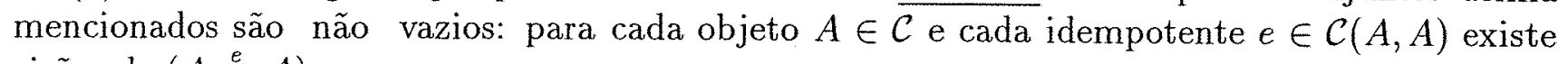
cisão de $(A \stackrel{e}{\rightarrow} A)$.

(3) Cisões de idempotentes são limites/colimites preservados por todos os funtores.

\footnotetext{
${ }^{18} \mathrm{O}$ uso do artigo definido é justificado pela unicidade a menos de isomorfismo.
} 


\section{Definição 1.46 .}

(i) Uma categoria $\mathcal{C}$ é completa se possuir limites para todos os $\mathcal{I}$-diagramas pequenos em $\mathcal{C}$; é cocompleta se possuir colimites para todos os $\mathcal{I}$-diagramas pequenos em $\mathcal{C}$.

Observamos que categorias completas, admitindo objetos finais são sempre não vazias. Dualmente, categorias cocompletas são não vazias porque admitem objeto inicial.

(ii) Há um análogo finito de completude : uma categoria $\mathcal{C}$ é finitamente completa se possuir limites para todos os $\mathcal{I}$-diagramas, onde $\mathcal{I}$ é uma categoria finita. De modo dual, definimos finitamente cocompleta.

O próximo resultado, devido a P. Freyd, dá um critério para completude e cocompletude de uma categoria :

Teorema 1.47. Uma categoria é completa sse tem produtos e equalizadores. Uma categoria é cocompleta sse possui coprodutos e coequalizadores. Resultados análogos valem para completude e cocompletude finitas.

Uma das propriedades mais importantes de funtores adjuntos é a presevação de limites ou colimites :

Teorema 1.48. Se F é um adjunto à esquerda, então $F$ preserva todos os colimites que existem no seu domínio. Dualmente, um adjunto à direita preserva todos os limites que existem em seu domínio.

Prova. Veja Teorema V.5.1, pag. 114 em [Mac].

Para categorias completas, temos caracterizações de funtores adjuntos e representáveis. Para apresentar estas duas importantes aplicações da preservação de limites, precisaremos da

Definição 1.49. Seja $F: \mathcal{A} \longrightarrow \mathcal{B}$ um funtor e $B \in \operatorname{Obj}(\mathcal{B})$. Um conjunto $S_{B}$ de objetos em $\mathcal{A}$ é um conjunto solução para $B$ com respeito a $F$ se para cada $A \in \mathcal{A}$ e cada morfismo $B \stackrel{f}{\longrightarrow} F(A)$, existe $A^{\prime} \in S_{B}$, juntamente com morfismos $A^{\prime} \stackrel{h}{\longrightarrow} A$ e $B \stackrel{f^{\prime}}{\longrightarrow} F\left(A^{\prime}\right)$, tais que o seguinte diagrama é comutativo:

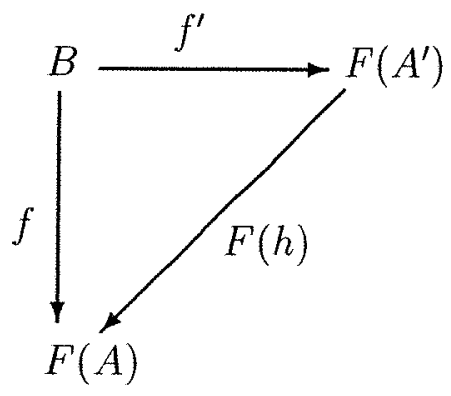


$F$ tem conjunto solução se todo $B \in \operatorname{Obj}(\mathcal{B})$ tem conjunto solução com respeito a $F$.

Teorema 1.50. (Teorema do Funtor Representável) Seja $\mathcal{A}$ uma categoria completa. Um funtor $F: \mathcal{A} \longrightarrow$ Set é representável sse $F$ preserva limites e $1=\{\emptyset\}$ tem um conjunto solução com respeito a $F$.

Teorema 1.51. (Teorema do Funtor Adjunto) Sejam $\mathcal{B}$ uma categoria completa e $G: \mathcal{B} \longrightarrow \mathcal{A}$ um funtor covariante. Então, $G$ tem adjunto à esquerda sse $G$ preserva limites e tem conjunto de solução.

\subsubsection{Categorias de Diagramas}

Nesta subseção apresentamos a noção de categoria dos diagramas e morfismos projetores associados a uma categoria dada e indexados por classe de categorias especificada, um conceito aparentado com o de pro-categoria, introduzido por Grothendieck (ver [GV]). A subseção 5.4.3 de [MP] é dedicada ao estudo da versão dual deste tema.

Fixemos $\Omega$ uma subcategoria da categoria Cat $^{19}$ e $\mathcal{C}$ uma categoria qualquer.

Definição 1.52. Denota-se $\operatorname{Diag}_{\Omega}(\mathcal{C})$ a categoria dos diagramas e morfismos projetores a valores em $\mathcal{C}$ indexados por categorias em $\Omega$ :

Objetos : pares $(\mathcal{I}, F)$ tais que $\mathcal{I} \in \Omega$ e $(\mathcal{I} \stackrel{F}{\rightarrow} \mathcal{C})$ é um funtor.

Flechas : um morfismo com dominio $(\mathcal{I}, F)$ e codomínio $\left(\mathcal{I}^{\prime}, F^{\prime}\right)$ é um par $(T, \tau)$ :

$$
\left((\mathcal{I}, F) \stackrel{(T, \tau)}{\rightarrow}\left(\mathcal{I}^{\prime}, F^{\prime}\right)\right) \in \operatorname{Diag}_{\Omega}(\mathcal{C}), \text { onde: }
$$

* $\left(\mathcal{I}^{\prime} \stackrel{T}{\rightarrow} \mathcal{I}\right)$ é um funtor ("de mudança de base");

* $\left(F \circ T \stackrel{\tau}{\rightarrow} F^{\prime}\right)$ é uma transformaçẫo natural ("de comparação") em $\operatorname{Nat}\left(\mathcal{I}^{\prime}, \mathcal{C}\right)$.

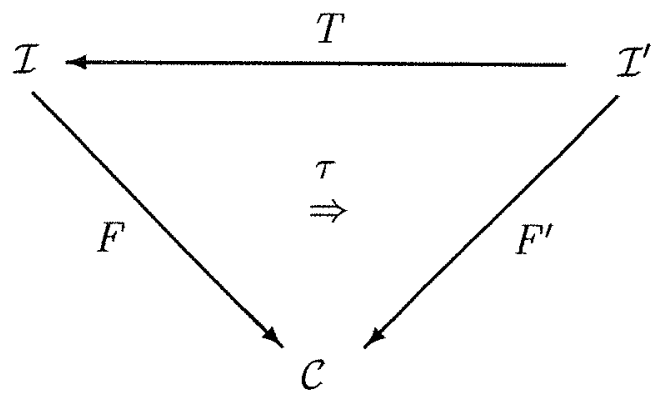

\footnotetext{
${ }^{19}$ Cat é a categoria cujos objetos são as categorias pequenas e cujos morfismos são funtores .
} 
Identidade $:\left(\left(\mathcal{I}^{\prime}, F^{\prime}\right) \stackrel{\mathbf{1}_{\left(I^{\prime}, F^{\prime}\right)}^{\rightarrow}}{\rightarrow}\left(\mathcal{I}^{\prime}, F^{\prime}\right)\right) \doteq\left(\left(\mathcal{I}^{\prime}, F^{\prime}\right) \stackrel{\left(1_{I^{\prime}}, 1_{F^{\prime}}\right)}{\rightarrow}\left(\mathcal{I}^{\prime}, F^{\prime}\right)\right)$.

A mudança de base da identidade é a identidade da base.

A transformação natural de comparação da identidade é a transformação identidade.

Composição $:\left((\mathcal{I}, F) \stackrel{(T, \tau)}{\rightarrow}\left(\mathcal{I}^{\prime}, F^{\prime}\right) \stackrel{\left(T^{\prime}, \tau^{\prime}\right)}{\rightarrow}\left(\mathcal{I}^{\prime \prime}, F^{\prime \prime}\right)\right) \doteq\left((\mathcal{I}, F) \stackrel{\left(T^{\prime \prime}, \tau^{\prime \prime}\right)}{\rightarrow}\left(\mathcal{I}^{\prime \prime}, F^{\prime \prime}\right)\right)$.

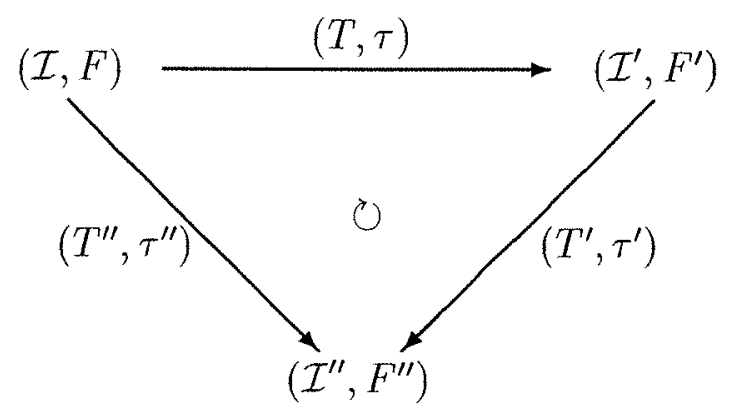

Diagrama em $\operatorname{Diag}_{\Omega}(\mathcal{C})$.

Onde:

* $\left(\mathcal{I}^{\prime \prime} \stackrel{T^{\prime}}{\rightarrow} \mathcal{I}^{\prime} \stackrel{T}{\rightarrow} \mathcal{I}\right) \doteq\left(\mathcal{I}^{\prime \prime} \stackrel{T^{\prime \prime}}{\rightarrow} \mathcal{I}\right):$

A mudança de base da composta é a composta das mudanças de base.

$$
T^{\prime \prime} \doteq T \circ T^{\prime}
$$

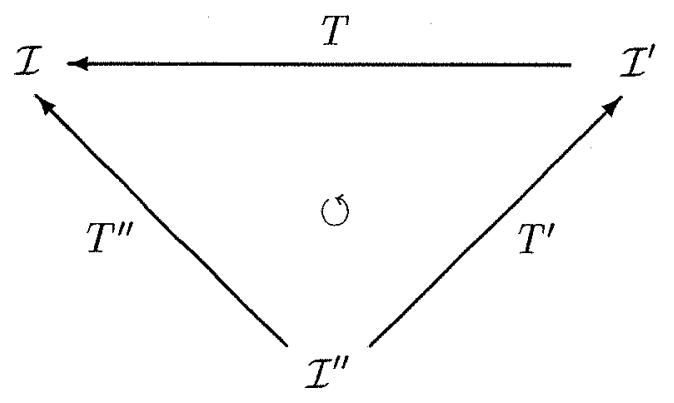

Diagrama em $\Omega$.

* $\left(F \circ T \circ T^{\prime} \stackrel{\tau T^{\prime}}{\rightarrow} F^{\prime} \circ T^{\prime} \stackrel{\tau^{\prime}}{\rightarrow} F^{\prime \prime}\right)=\left(F \circ T^{\prime \prime} \stackrel{\tau^{\prime \prime}}{\rightarrow} F^{\prime \prime}\right)$

$$
\tau^{\prime \prime}=\tau^{\prime} \circ\left(\tau T^{\prime}\right) \in \operatorname{Nat}\left(\mathcal{I}^{\prime \prime}, \mathcal{C}\right) \text {. }
$$

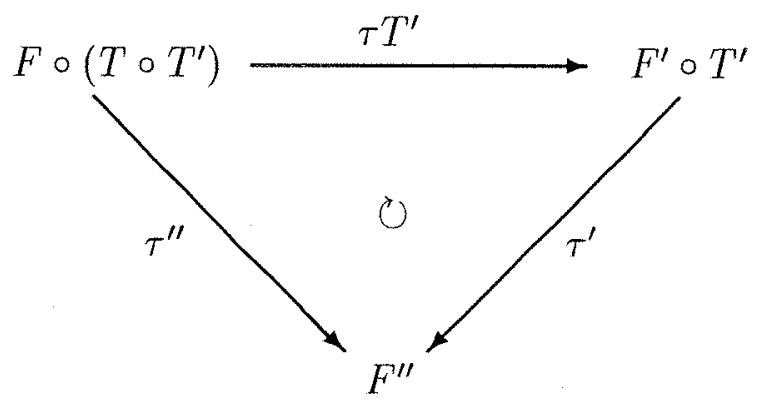

Diagrama em $\left[\mathcal{I}^{\prime \prime}, C\right]$. 
Observação 1.53. A cada funtor contravariante $\mathcal{T}: \mathcal{C}^{o p} \rightarrow \Omega$ podemos associar um funtor "diagonal" : $\delta_{\mathcal{T}}: \mathcal{C} \rightarrow \operatorname{Diag}_{\Omega}(\mathcal{C}):$

$$
\left\{\begin{array}{l}
A \mapsto \delta_{\mathcal{T}_{(A)}}: \mathcal{T}(A) \longrightarrow \mathcal{C}:\left(f \in[i, j]_{\mathcal{T}_{(A)}} \mapsto\left(A \stackrel{I d_{A}}{\longrightarrow} A\right)\right) \\
\left(A \stackrel{h}{\longrightarrow} A^{\prime}\right) \mapsto\left(\mathcal{T}(h), \delta_{\mathcal{T}_{\left(A^{\prime}\right)}}(h)\right)
\end{array}\right.
$$

Proposição 1.54. Seja $\left((\mathcal{I}, F) \stackrel{(T, \tau)}{\rightarrow}\left(\mathcal{I}^{\prime}, F^{\prime}\right)\right)$ um morfismo em $\operatorname{Diag}_{\Omega}(\mathcal{C})$.

(i) Temos um funtor $(T, \tau)_{\star}:$ Cone $(\mathcal{I} \stackrel{F}{\rightarrow} \mathcal{C}) \longrightarrow \operatorname{Cone}\left(\mathcal{I}^{\prime} \stackrel{F^{\prime}}{\rightarrow} \mathcal{C}\right)$.

(ii) Se $\mathcal{C}$ é uma categoria $\Omega$-completa ${ }^{20}$ então existe $\mathcal{C}$-morfismo canônico $\underset{i \in I}{\stackrel{\lim }{\leftarrow}} F(i) \rightarrow \underset{i^{\prime} \in I^{\prime}}{\longleftrightarrow} F^{\prime}\left(i^{\prime}\right)$.

\section{Prova.}

(i) $(T, \tau)_{\star}:$ Cone $(\mathcal{I} \stackrel{F}{\rightarrow} \mathcal{C}) \longrightarrow \operatorname{Cone}\left(\mathcal{I}^{\prime} \stackrel{F^{\prime}}{\rightarrow} \mathcal{C}\right)$ é simplesmente a composição dos funtores descritos em 1.43 :

$$
\begin{aligned}
T_{\star}: \text { Cone }(\mathcal{I} \stackrel{F}{\rightarrow} \mathcal{C}) \longrightarrow \operatorname{Cone}\left(\mathcal{I}^{\prime} \stackrel{F \circ T}{\rightarrow} \mathcal{C}\right) \\
\tau_{\star}: \text { Cone }\left(\mathcal{I}^{\prime} \stackrel{F \circ T}{\rightarrow} \mathcal{C}\right) \rightarrow \operatorname{Cone}\left(\mathcal{I}^{\prime} \stackrel{F^{\prime}}{\rightarrow} \mathcal{C}\right)
\end{aligned}
$$

Notemos que, como tanto $T_{\star}$ e $\tau_{\star}$ não alteram vértices e morfismos de cones (1.43), temos a mesma propriedade para o funtor $(T, \tau)_{\star}$.

(ii) Por hipótese, os diagramas $(\mathcal{I} \stackrel{F}{\rightarrow} \mathcal{C})$ e $\left(\mathcal{I}^{\prime} \stackrel{F^{\prime}}{\rightarrow} \mathcal{C}\right)$ admitem cones limites, respectivamente $\lim F$ e $\lim F^{\prime}$. Aplicando o funtor $(T, \tau)_{\star}$ do item (i) ao cone limite lim $F$ de base $F$ obtemos um cone de base $F^{\prime}$ logo, pela propriedade universal do cone $\lim F^{\prime} \overleftarrow{\text { de }}$ base $F^{\prime}$, existe um único morfismo de cones de base $F^{\prime}:(T, \tau)_{\star}\left(\lim _{\leftarrow} F\right) \rightarrow \lim F^{\prime}$. O $\mathcal{C}$-morfismo referido $\lim _{i \in I} F(i) \longrightarrow \lim _{i^{\prime} \in I^{\prime}}^{\lim } F^{\prime}\left(i^{\prime}\right)$ é o tal morfismo de cones.

\section{Proposição $\mathbf{1 . 5 5 .}$}

(i) A associação $(T, \tau) \mapsto(T, \tau) \star$ descrita na proposição acima é "funtorial" . Mais precisamente:

* Seja $\left((\mathcal{I}, F) \stackrel{I d_{(I, F)}}{\rightarrow}(\mathcal{I}, F)\right)$ o morfismo identidade de $(\mathcal{I}, F) \in \operatorname{Diag}_{\Omega}(\mathcal{C})$ então :

$$
\text { Temos funtor identidade } I d_{(I ; F)_{\star}}=I d_{\text {Cone }(F)}: \text { Cone }(\mathcal{I} \stackrel{F}{\rightarrow} \mathcal{C}) \rightarrow \operatorname{Cone}(\mathcal{I} \stackrel{F}{\rightarrow} \mathcal{C})
$$

\footnotetext{
${ }^{20}$ Isto é, todo diagrama indexado por alguma categoria em $\Omega$ e a valores em $\mathcal{C}$ admite cone limite.
} 
* Seja $\left((\mathcal{I}, F) \stackrel{\left(T^{\prime \prime}, \tau^{\prime \prime}\right)}{\rightarrow}\left(\mathcal{I}^{\prime \prime}, F^{\prime \prime}\right)\right)$ o morfismo composto $\left((\mathcal{I}, F) \stackrel{(T, \tau)}{\rightarrow}\left(\mathcal{I}^{\prime}, F^{\prime}\right) \stackrel{\left(T^{\prime}, \tau^{\prime}\right)}{\rightarrow}\left(\mathcal{I}^{\prime \prime}, F^{\prime \prime}\right)\right)$ então :

Temos funtor composto $\left(T^{\prime \prime}, \tau^{\prime \prime}\right)_{\star}=\left(T^{\prime}, \tau^{\prime}\right)_{\star} \circ(T, \tau)_{\star}:$ Cone $(\mathcal{I} \stackrel{F}{\rightarrow} \mathcal{C}) \rightarrow$ Cone $\left(\mathcal{I}^{\prime \prime} \stackrel{F^{\prime \prime}}{\rightarrow} \mathcal{C}\right)$.

(ii) Se $\mathcal{C}$ é uma categoria $\Omega$-completa e fixamos escolha global de cones limites sobre diagramas

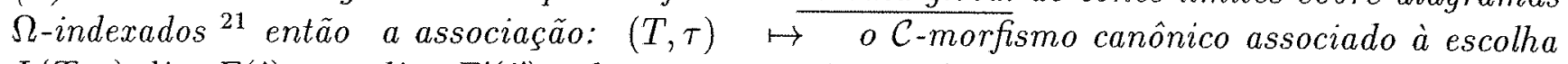
$L(T, \tau) \underset{i \in I}{\lim } F(i) \longrightarrow \underset{i^{\prime} \in I^{\prime}}{\lim } F^{\prime}\left(i^{\prime}\right)$, determina um funtor ("limite") $L: \operatorname{Diag}_{\Omega}(\mathcal{C}) \longrightarrow \mathcal{C}:$

* OC-morfismo induzido pela identidade

$$
\left((\mathcal{I}, F) \stackrel{I d_{(I, F)}}{\longrightarrow}(\mathcal{I}, F)\right)
$$

é a identidade

$$
L\left(I d_{(I, F)}\right)=I d_{\lim F}: \lim F \rightarrow \lim F
$$

* OC-morfismo induzido pela composição

$$
\left((\mathcal{I}, F) \stackrel{\left(T^{\prime \prime}, r^{\prime \prime}\right)}{\longrightarrow}\left(\mathcal{I}^{\prime \prime}, F^{\prime \prime}\right)\right)=\left((\mathcal{I}, F) \stackrel{(T, \tau)}{\longrightarrow}\left(\mathcal{I}^{\prime}, F^{\prime}\right) \stackrel{\left(T^{\prime}, \tau^{\prime}\right)}{\longrightarrow}\left(\mathcal{I}^{\prime \prime}, F^{\prime \prime}\right)\right)
$$

é a composição

$$
L\left(T^{\prime \prime}, \tau^{\prime \prime}\right)=L\left(T^{\prime}, \tau^{\prime}\right) \circ L(T, \tau): \lim F \rightarrow \lim F^{\prime \prime}
$$

\subsubsection{Objetos Injetivos e Projetivos}

Definição 1.56. Sejam $\mathcal{C}$ uma categoria, $\Gamma \subseteq \mathcal{M}(\mathcal{C})$ e $C \in \operatorname{Obj}(\mathcal{C})$.

(i) $C$ é $\Gamma$-projetivo em $\mathcal{C}$ se para todo $A, B$ em $O b j(\mathcal{C})$, todo morfismo em $\Gamma A \stackrel{f}{\longrightarrow} B$ e todo morfismo $C \stackrel{g}{\longrightarrow} B$, existe $C \stackrel{h}{\longrightarrow}$ A tal que $f \circ h=g$.
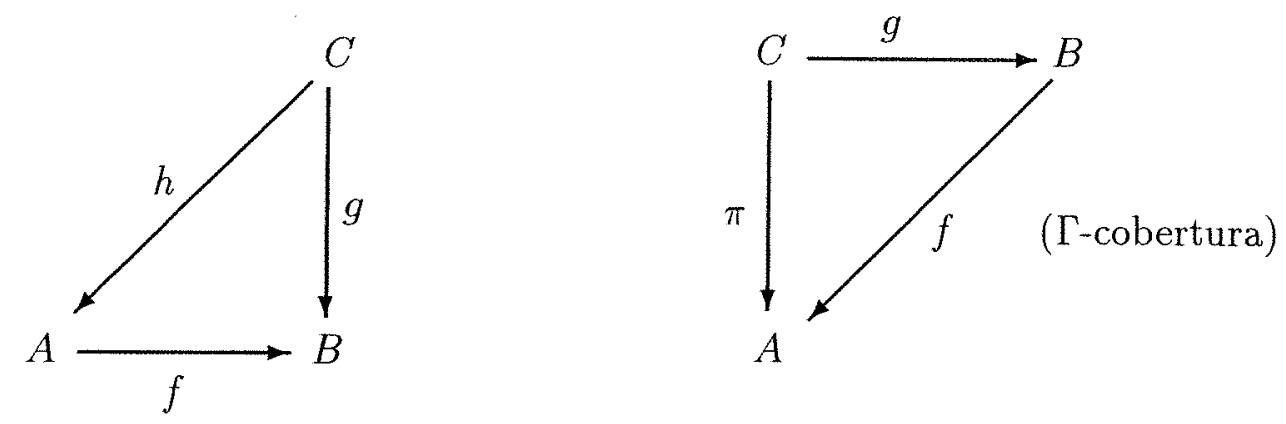

(ii) $C$ é uma cobertura $\Gamma$-projetiva de $A$ se $C$ é um objeto $\Gamma$-projetivo e existe um morfismo em $\Gamma, C \stackrel{\pi}{\longrightarrow} A$, tal que para qualquer $\Gamma$-projetivo $B$ em $\mathcal{C}$ e todo morfismo em $\Gamma \quad B \stackrel{f}{\longrightarrow} A$, existe um morfismo em $\Gamma \quad C \stackrel{g}{\longrightarrow} B$ tal que $f \circ g=\pi$.

\footnotetext{
${ }^{21}$ Por exemplo, aos diagramas constantes associamos o cone "identidade". Notamos que assim como a categoria Set possui limites e colimites canônicos, diversas categorias construídas a partir de Set (como Top, L-mod, ...) possuem escolhas canônicas de limites e colimites.
} 
$\mathcal{C}$ tem suficientes $\Gamma$-projetivos se para todo objeto $A$ em $\mathcal{C}$, existe um $\Gamma$-projetivo $C$ e um morfismo em $\Gamma \quad C \stackrel{f}{\rightarrow} A$.

Quando tomamos a classe $\Gamma \subseteq \mathcal{M}(\mathcal{C})$ como a classe de todos os epimorfismos de $\mathcal{C}$ então dizemos apenas projetivos e cobertura projetiva.

(iii) $C$ é $\Gamma$-injetivo em $\mathcal{C}$ se para todo $A, B$ em $O b j(\mathcal{C})$, todo morfismo em $\Gamma A \stackrel{f}{\longrightarrow} B$ e todo morfismo $A \stackrel{g}{\longrightarrow} C$, existe um morfismo $B \stackrel{h}{\longrightarrow} C$ tal que $h \circ f=g$.
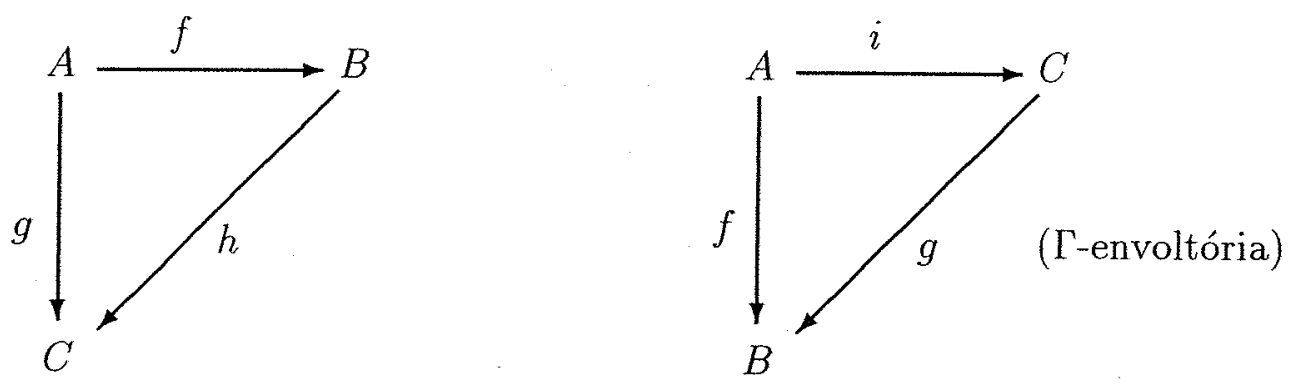

(iv) $C$ é uma envoltória $\Gamma$-injetiva de $A$ se $C$ é um objeto $\Gamma$-injetivo e existe um morfismo em $\Gamma, A \stackrel{i}{\longrightarrow} C$, tal que para qualquer $\Gamma$-injetivo $B$ em $\mathcal{C}$ e todo morfismo em $\Gamma A \stackrel{f}{\longrightarrow} B$, existe um morfismo em $\Gamma \quad C \stackrel{g}{\rightarrow} B$ tal que $g \circ i=f$.

$\mathcal{C}$ tem suficientes $\Gamma$-injetivos se para todo objeto $A$ em $\mathcal{C}$ existe um $\Gamma$-injetivo $C$ e um morfismo em $\Gamma \stackrel{f}{\longrightarrow} C$.

Quando tomamos a classe $\Gamma \subseteq \mathcal{M}(\mathcal{C})$ como a classe de todos os monomorfismos de $\mathcal{C}$ então dizemos apenas injetivos e envoltória injetiva.

Observação 1.57. É imediato que:

* Coprodutos de $\Gamma$-projetivos são $\Gamma$-projetivos; retratos de $\Gamma$-projetivos são $\Gamma$-projetivos.

* Produtos de $\Gamma$-injetivos são $\Gamma$-injetivos; retratos de $\Gamma$-injetivos são $\Gamma$-injetivos.

Como um exemplo, mencionaríamos que na categoria das álgebras de Heyting, bem como na das álgebras de Boole, os objetos injetivos são as álgebras de Boole completas. Além disso, o completado de uma álgebra de Boole é a sua envoltória injetiva. Estes fatos não são imediatos, dependendo de um resultado bem conhecido de Sikorski acerca da extensão de morfismos com valores em álgebras de Boole completas. 


\section{Capítulo 2}

\section{Limites e Colimites de Estruturas de Primeira Ordem}

Neste Capítulo apresentaremos alguns fatos fundamentais sobre linguagens e estruturas de primeira ordem. Além disso, incluímos uma discussão de limites e colimites na categoria das estruturas de primeira ordem, que embora seja folclore na Teoria dos Modelos, não parece haver uma referência conveniente para o assunto na literatura. A última seção contém a prova de um dos resultados fundamentais deste trabalho, o Teorema 2.50 , que garante que $L$-estruturas profinitas ${ }^{1}$ são retratos de certos ultraprodutos de estruturas finitas.

Assumiremos familiaridade com as noções fundamentais da Lógica e da Teoria dos Modelos. Referências clássicas para este assunto são $[\mathbf{K l}],[\mathbf{K l 1}],[\mathbf{S c h}],[\mathbf{C K}]$ e $[\mathbf{H o}] ;[\mathbf{S m}]$ traz uma visão interessante sobre a Lógica de Primeira Ordem.

\subsection{Linguagens e Lógicas de Primeira Ordem}

Lembramos a construção de uma linguagem de primeira ordem com igualdade, $L$.

\subsection{Alfabeto de $L$ :}

* Um conjunto $\left\{v_{n}: n \in \mathbb{N}\right\}$ de símbolos para variáveis;

* Os símbolos lógicos $\wedge$ (e), $\vee$ (ou), $\rightarrow$ (implica) e $\neg$ (negação);

* Os quantificadores $\exists$ (existencial) e $\forall$ (universal);

* Um símbolo relacional binário, $=$, representando a igualdade;

* Para cada natural $n \geq 1$ :

** Um conjunto $\operatorname{rel}(n, L)$ de símbolos relacionais $n$-ários.

** Um conjunto op $(n, L)$ de símbolos funcionais $n$-ários.

** Um conjunto $c t(L)$ de símbolos para constantes.

\footnotetext{
${ }^{1}$ Isto é, limites projetivos cofiltrantes de estruturas finitas.
} 
2.2. Termos : São definidos por indução na complexidade, onde $n \geq 1$ é um natural :

* Variáveis e constantes são termos.

* Se $t_{1}, \ldots, t_{n}$ são termos e $\omega \in o p(n)$, então $\omega\left(t_{1}, \ldots, t_{n}\right)$ é um termo.

2.3. Fórmulas : São definidas por indução na complexidade, onde $n \geq 1$ é um natural : * Se $R \in \operatorname{rel}(n)$ e $t_{1}, \ldots, t_{n}$ são termos em $L, R\left(t_{1}, \ldots, t_{n}\right)$ é uma fórmula, denominada atômica. $* \operatorname{Se} \varphi, \psi$ são fórmulas em $L$ e $\diamond$ é um dos símbolos $\wedge, \vee$ ou $\rightarrow$, então $(\varphi \diamond \psi)$ é uma fórmula. * Se $\varphi$ é uma fórmula, então $\neg \varphi$ é uma fórmula.

* Se $\varphi$ é uma fórmula e $v_{n}$ é uma variável, então $\forall v_{n} \varphi$ e $\exists v_{n} \varphi$ são fórmulas.

\subsection{Noções Básicas :}

a) A cada fórmula $\varphi$ em $L$ está associada uma sequência de fórmulas, de complexidade menor, que descrevem o processo de construção de $\varphi$ a partir das atômicas. As fórmulas nesta sequência denominam-se subfórmulas de $\varphi$. As ocorrências de uma variável $v_{n}$ em $\varphi$ dividem-se em duas categorias :

(1) ligadas, quando $v_{n}$ ocorre em uma subfórmula de $\varphi$ do tipo $Q v_{n} \psi$, onde $Q=\exists, \forall$;

(2) livre, no caso em que a ocorrência não é ligada.

b) (Substituição) Se $\varphi$ é uma fórmula, $v_{n}$ é uma variável e $\tau$ é um termo, indicamos por $\varphi\left(\left\ulcorner\tau \mid v_{n}\right\urcorner\right)$ a fórmula obtida substituindo-se todas as ocorrências livres de $v_{n}$ em $\varphi$ por $\tau$.

c) Se $t$ é um termo e $\varphi$ é uma fórmula, escrevemos $t\left(v_{1}, \ldots, v_{n}\right), \varphi\left(v_{1}, \ldots, v_{n}\right)$ para indicar que as variáveis livres $t$ e $\varphi$ estão entre as $v_{1}, \ldots, v_{n}$. Como é usual, escrevemos $\bar{v}$ para indicar a sequência $\left\langle v_{1}, \ldots, v_{n}\right\rangle$; poderemos também, como é standard, utilizar $x, \bar{x}, y, \bar{y}$ e $z$, $\bar{z}$ para indicar variáveis.

d) Um termo $t$ é livre para $\boldsymbol{v}_{n}$ em uma fórmula $\varphi$ sse em $\varphi\left(\left\ulcorner t \mid v_{n}\right\urcorner\right.$ ) (veja (b) acima) nenhuma variável em $t$ se torna ligada.

e) Uma fórmula em $\varphi$ em $L$ é

* aberta se possuir alguma variável ocorrendo livre;

* sentença se não for aberta; denota-se $\operatorname{Sent}(L)$ o conjunto das sentenças em $L$;

* livre de quantificadores se não há ocorrência de quantificadores em $\varphi$;

* positiva se os símbolos de implicação e negação não ocorrem em $\varphi$;

* universal se é equivalente a uma fórmula do tipo $\forall \bar{v} \varphi$, onde $\varphi$ é livre de quantificadores;

* existencial se é equivalente a uma fórmula do tipo $\exists \bar{v} \varphi$, onde $\varphi$ é livre de quantificadores; 
* existencial positiva (e.p.) se for construída a partir das atômicas utilizando somente os conectivos $\wedge, \vee$ e o quantificador existencial;

* positiva primitiva (p.p.) se é da forma $\exists \bar{x} \varphi$, onde $\varphi$ é uma conjunção de fórmulas atômicas;

$* \boldsymbol{\Pi}_{n}\left(\right.$ respec. $\left.\boldsymbol{\Sigma}_{n}\right)$ se for da forma $\forall \overline{x_{0}} \exists \overline{x_{1}} \ldots Q \overline{x_{n}} \psi\left(\right.$ respec. $\exists \overline{x_{0}} \forall \overline{x_{1}} \ldots Q \overline{x_{n}} \psi$ ), onde $\psi$ é livre de quantificadores;

* Horn básica se for da forma $\forall \bar{x}\left[\left(\psi_{1} \wedge \ldots \wedge \psi_{n}\right) \rightarrow \psi\right]$, onde $\psi_{1}, \ldots, \psi_{n}$ são atômicas e $\psi$ é atômica ou uma fórmula logicamente equivalente à fórmula $\left(v_{0} \neq v_{0}\right)$.

2.5. Os Cálculos de Predicados Intuicionista e Clássico. A seguir apresentamos uma formalização no estilo Hilbert, ${ }^{2}$ do Cálculo de Predicados Intuicionista com igualdade ${ }^{3}$, indicado por $\mathcal{H}$ :

Se $\varphi, \psi$ e $\chi$ são fórmulas em $L, v$ é uma variável e $\tau$ é um termo :

1. $\varphi \rightarrow(\psi \rightarrow \varphi)$

2. $(\varphi \rightarrow \psi) \rightarrow((\varphi \rightarrow(\psi \rightarrow \chi)) \rightarrow(\varphi \rightarrow \chi))$;

3. $\varphi \rightarrow(\psi \rightarrow \varphi \wedge \psi)$;

4. $\varphi \wedge \psi \rightarrow \varphi$;

5. $\varphi \wedge \psi \rightarrow \psi$

6. $\varphi \rightarrow(\varphi \vee \psi)$;

7. $\psi \rightarrow(\varphi \vee \psi)$;

8. $(\varphi \rightarrow \chi) \rightarrow((\psi \rightarrow \chi) \rightarrow(\varphi \vee \psi \rightarrow \chi))$;

9. $(\varphi \rightarrow \psi) \rightarrow((\varphi \rightarrow \neg \psi) \rightarrow \neg \varphi)$;

10. $\neg \varphi \rightarrow(\varphi \rightarrow \psi)$.

11. Se $\tau$ é livre para $v \operatorname{em} \varphi,{ }^{4}\left\{\begin{array}{l}\text { 11.a. } \forall v \varphi \rightarrow \varphi(\ulcorner\tau \mid v\urcorner) ; \\ \text { 11.b. } \varphi(\ulcorner\tau \mid v\urcorner) \rightarrow \exists v \varphi,\end{array}\right.$ onde $\varphi(\ulcorner\tau \mid v\urcorner)$ é a substituição de $\tau$ por $v \operatorname{em} \varphi$, como em 2.4.(b).

12. Regras de Dedução :

$$
\text { Modus Ponens : } \frac{\varphi, \varphi \rightarrow \psi}{\psi} \quad \begin{cases}\text { Regra } \forall: \frac{\varphi \rightarrow \psi(v)}{\varphi \rightarrow \forall v \psi(v)} \\ \text { Regra } \exists: \frac{\psi(v) \rightarrow \varphi}{\exists v \psi(v) \rightarrow \varphi}\end{cases}
$$

onde nas regras dos quantificadores $v$ não pode ocorrer livre em $\varphi$.

\footnotetext{
${ }^{2}$ para formalizações no estilo Gentzen, veja [ $\left.\mathbf{P r}\right],[\mathbf{K l 1}],[\mathbf{K l}]$.

${ }^{3}$ Devida a A. Heyting.

${ }^{4}$ Como em 2.4.(d).
} 
Os axiomas da igualdade são os usuais, incluindo a regra de Leibniz [L] : Se $\tau$ é um termo em $L$, livre para $v \operatorname{em} \varphi$, então

$$
\varphi(v) \wedge(v=\tau) \rightarrow \varphi(\ulcorner\tau \mid v\urcorner) .
$$

Os primeiros dez esquemas, juntamente com Modus Ponens formalizam o Cálculo Proposicional Intuicionista. Para obter o Cálculo Clássico, adicionamos o (ou substituímos o axioma 10 pelo) esquema

$$
10^{C} . \neg \neg \varphi \rightarrow \varphi .
$$

onde $\varphi$ é uma fórmula qualquer em $L$. Em [Sc] há uma proposta diferente e interessante para a formalização do Cálculo de Predicados Intuicionista.

Se $\Gamma \cup\{\varphi\}$ é um conjunto de fórmulas em $L$, uma prova de $\varphi$ a partir de $\Gamma$ consiste de uma sequência de fórmulas $\psi_{1}, \ldots, \psi_{n}$ em $L$, tal que $\psi_{n}$ é $\varphi$ e verificando, para $1 \leq k \leq n$,

$* \psi_{k} \in \Gamma \quad$ ou $\quad * \psi_{k}$ é uma axioma ou

* $\psi_{k}$ vem de fórmulas anteriores na sequência pela aplicação de uma das regras de dedução.

Escrevemos

$$
\begin{cases}\Gamma \vdash_{\mathcal{H}} \varphi & \text { Se } \varphi \text { consequência de } \Gamma \text { em } \mathcal{H} \\ \Gamma \vdash_{\mathcal{C}} \varphi & \operatorname{Se} \varphi \text { é consequência de } \Gamma \text { no Cálculo Clássico. }\end{cases}
$$

Está claro que $\Gamma \vdash_{\mathcal{H}} \varphi \Rightarrow \Gamma \vdash_{\mathcal{C}} \varphi$. Na definição abaixo o símbolo $\vdash_{\text {indica }} \vdash_{\mathcal{H}}$ e $\vdash_{\mathcal{C}}$.

Definição 2.6. Seja $\Gamma \cup\{\varphi\}$ um conjunto de fórmulas em L. O símbolo $\vdash$ indica $\vdash_{\mathcal{H}} e \vdash_{\mathcal{C}}$.

a) $\Gamma$ é inconsistente se para alguma fórmula $\psi \mathrm{em} L, \Gamma \vdash \psi \wedge \neg \psi$.

b) $\Gamma$ é consistente se não for inconsistente.

c) $\varphi$ é um teorema $($ de $\mathcal{H}$ ou $\mathcal{C})$ se $\varphi \vdash \varphi$, em geral indicado por $\vdash \varphi$.

d) $\Gamma$ é uma teoria $(e m \mathcal{H}$ ou $\mathcal{C})$ se for um conjunto de sentenças tal que para toda $\sigma \in \operatorname{Sent}(L)$

$$
\Gamma \vdash \sigma \Rightarrow \sigma \in \Gamma \text {, }
$$

ou seja, $\Gamma$ é fechado em relação à consequência lógica.

Um dos resultados importantes nestes sistemas é o:

Proposição 2.7. (Teorema da Dedução) Seja $\Gamma \cup\{\sigma, \varphi\}$ um conjunto de fórmulas em L. Se $\sigma$ é uma sentença, então

$$
\Gamma, \sigma \vdash_{\mathcal{H}} \varphi \quad \Leftrightarrow \quad \Gamma \vdash_{\mathcal{H}}(\sigma \rightarrow \varphi) .
$$

Um resultado análogo vale para a relação $\vdash_{\mathcal{C}}$. 
Observação 2.8. Seja $L$ uma linguagem de primeira ordem com igualdade. Como as noções de fórmula existencial positiva e positiva primitiva (2.4.(e)) aparecerão com certa frequência neste trabalho, utilizaremos uma notação especial para estes tipos de fórmulas, a saber :

* $\exists^{+}(L)$ indica o conjunto das fórmulas de $L$ que são logicamente equivalentes (no Cálculo Clássico) a uma fórmula existencial positiva;

* $\operatorname{pp}(L)$ indica o conjunto das fórmulas de $L$ que são logicamente equivalentes (no Cálculo Clássico) a uma fórmula positiva primitiva.

A relação entre os conjuntos $\exists^{+}(L)$ e pp $(L)$ está descrita no seguinte:

Lema 2.9. Se $\varphi \in \exists^{+}(L)$, então existem subconjuntos finitos $P_{1}, \ldots, P_{n}$ de $\operatorname{pp}(L)$ tais que

$$
\vdash_{\mathcal{C}} \varphi \leftrightarrow\left(\psi_{1} \vee \psi_{2} \vee \ldots \vee \psi_{n}\right)
$$

onde $\psi_{j}$ é a conjunção das fórmulas em $P_{j}, 1 \leq j \leq n$.

Prova. (Esboço) Como a equivalência entre fórmulas é transitiva, é suficiente mostrar que o resultado vale para uma fórmula $\varphi$ construída a partir das atômicas utilizando conjunção, disjunção e o quantificador existencial. Neste caso, a prova é por indução na complexidade de $\varphi$ ${ }^{5}$, lembrando que se $\psi(x)$ e $\chi(x)$ são fórmulas então

$$
\vdash_{\mathcal{C}} \exists x(\psi(x) \vee \chi(x)) \leftrightarrow \exists x \psi(x) \vee \exists x \chi(x)
$$

Uma das descobertas importantes de Lindenbaum e Tarski é que a sintaxe de teorias formais gera estruturas algébricas interessantes. $\operatorname{Se} \varphi, \psi$ são fórmulas em $L$, definimos a relação

$$
\varphi \equiv \psi \quad \Leftrightarrow \quad \vdash_{\mathcal{H}}(\varphi \rightarrow \psi) \wedge(\psi \rightarrow \varphi)
$$

Esta é uma relação de equivalência entre as fórmulas de $L$ e o conjunto das suas classes de equivalência é a álgebra de Lindenbaum de $L$. Para sistemas intuicionistas - proposicionais ou de primeira ordem -, esta álgebra é uma álgebra de Heyting; para os sistemas clássicos, é uma álgebra de Boole. Para mais detalhes, recomendamos [Ra] e [RS].

\subsection{Estruturas de Primeira Ordem e seus Morfismos}

Como sequências serão de uso constante, faremos a seguinte convenção notacional :

\subsection{Notação :}

a) Se $X_{i}, i \in I$, é uma família de conjuntos e $X=\prod_{i \in I} X_{i}$ o seu produto cartesiano. Iremos indicar os elementos de $X$ por

\footnotetext{
${ }^{5}$ Está claro que toda fórmula atômica é pp.
} 


$$
x: I \longrightarrow \bigcup_{i \in I} X_{i},\left\langle x_{i}\right\rangle_{i \in I} \text { ou }\langle x(i)\rangle_{i \in I}
$$

conforme mais conveniente para facilitar a leitura.

b) Sejam $A, B$ conjuntos e $n \geq 1$ um natural.

* Escrevemos $\bar{x}=\left\langle x_{1}, \ldots, x_{n}\right\rangle$ para indicar um elemento típico de $A^{n}$;

* Se $f: A \longrightarrow B$ é uma função e $\bar{x} \in A^{n}$, definimos

$$
f(\bar{x})=\left\langle f\left(x_{1}\right), \ldots, f\left(x_{n}\right)\right\rangle .
$$

Note que isto nada mais é do que uma notação conveniente para a função $f^{n}: A^{n} \longrightarrow B^{n}$, naturalmente induzida por $f$.

2.11. L-estruturas : Seja $L$ uma linguagem de primeira ordem com igualdade, como na seção anterior. Uma $L$-estrutura é um conjunto $M$, juntamente com

* Para $R \in \operatorname{rel}(n, L)$, um subconjunto $R^{M} \subseteq M^{n}$, denominado a interpretação de $R$ em $M$;

* Para $\omega \in o p(n, L)$, uma função $\omega^{M}: M^{n} \longrightarrow M$, a interpretação de $\omega$ em $M$;

* Para $c \in c t(L)$, um elemento $c^{M} \in M$, a interpretação de $c$ em $M$;

* O símbolo de igualdade é interpretado pela diagonal do produto $M^{2}=M \times M$.

Por indução na complexidade, um termo $t\left(v_{1}, \ldots, v_{n}\right)$, nas variáveis livres $v_{1}, \ldots, v_{n}$, induz uma função $t^{M}: M^{n} \longrightarrow M$, denominada a interpretação de $t$ em $M$.

Assumimos familiaridade com o conceito de interpretação de uma fórmula $\varphi\left(v_{1}, \ldots, v_{n}\right)$ em $M$ (veja por exemplo $[\mathbf{C K}]$ ) e do símbolo

$$
M \vDash \varphi[\bar{a}],
$$

que indica que $M$ satisfaz $\varphi$ na $n$-upla $\bar{a} \in M^{n}$.

Denotaremos $[\varphi]_{M} \doteq\left\{\bar{a} \in M^{n}: M \models \varphi[\bar{a}]\right\}$, o conjunto das n-uplas de $M$ que satisfazem $\varphi$.

Indicamos $L-\bmod$ a classe das $L$-estruturas.

Definição 2.12. Para cada L linguagem de primeira ordem com igualdade, a relação $\models$ de satisfação entre L-estruturas e L-sentenças induz associações (decrescentes) entre classes de L-estruturas e conjuntos de L-sentenças :

* Para cada $\Sigma \subseteq$ Sent $(L)$ conjunto de sentenças em $L$, indicamos por $\operatorname{Mod}(\Sigma)$ a classe das L-estruturas que são modelos de $\Sigma$, isto é,

$$
\operatorname{Mod}(\Sigma)=\{M: M \text { é L-estrutura e } M \vDash \sigma, \forall \sigma \in \Sigma\} .
$$

Uma classe de L-estruturas $\mathcal{K}$ é dita L-axiomatizável quando existe $\Sigma \subseteq \operatorname{Sent}(L)$ tal que $\mathcal{K}=$ $\operatorname{Mod}(\Sigma)$.

* Para cada $\mathcal{K} \subseteq L-\bmod$ classe de estruturas em $L$, indicamos por $\mathrm{Th}(\mathcal{K})$ o conjunto das $L$-sentenças que estão satisfeitas em $\mathcal{K}$, isto é, 


$$
\operatorname{Th}(\mathcal{K})=\{\sigma: \sigma e ́ \text { L-sentença e } M \vDash \sigma, \forall M \in \mathcal{K}\} .
$$

Um conjunto de L-sentenças $\Sigma$ é dito L-satisfazivel quando existe $\mathcal{K} \subseteq L$-mod tal que $\operatorname{Th}(\mathcal{K})=$ $\Sigma$.

Se $M$ e $N$ são L-estruturas, dizemos que $M$ e $N$ são elementarmente equivalentes, em simbolos $M \equiv N$, se $\operatorname{Th}(\{M\})=\operatorname{Th}(\{N\})$. É fácil verificar que esta condição é equivalente a

$$
\forall \sigma \in \operatorname{Sent}(L), \quad M \vDash \sigma \Rightarrow N \vDash \sigma .
$$

Um dos resultados mais fundamentais da Teoria dos Modelos é o seguinte:

Teorema 2.13. (Completude e Compacidade) Para $\Sigma \subseteq \operatorname{Sent}(L)$ as seguintes condições são equivalentes:

(1) $\Sigma$ é consistente (2.6.(a));

(2) Para todo $S \subseteq f \Sigma, \operatorname{Mod}(S) \neq \emptyset ;{ }^{6}$

(3) $\operatorname{Mod}(\Sigma) \neq \emptyset$.

Prova. Veja Teoremas 1.3.21 e 1.3.22 (pag. 66) em [CK].

As principais noções de morfismos de $L$-estruturas aparecem na

Definição 2.14. Seja $L$ uma linguagem de primeira ordem com igualdade e sejam $M, N L$ estruturas. Seja $f: M \longrightarrow N$ uma função.

a) fé um $\mathbf{L}$-morfismo se para todo natural $n \geq 1$

(i) Se $c \in \operatorname{ct}(L)$, então $f\left(c^{M}\right)=c^{N}$.

(ii) Se $\omega \in \operatorname{op}(n, L)$ e $\bar{x} \in M^{n}$, então $f\left(\omega^{M}(\bar{x})\right)=\omega^{N}(f(\bar{x}))$;

(iii) Se $R \in \operatorname{rel}(n, L)$ e $\bar{x} \in M^{n}$, então

$$
M \vDash R[\bar{x}] \Rightarrow N \models R[f(\bar{x})] .
$$

b) $f$ é uma $\boldsymbol{L}$-imersão (ou $\boldsymbol{L}$-monomorfismo) se for um $L$-morfismo tal que, para todo $n \geq$ 1, cada $R \in \operatorname{rel}(n, L)$ e cada $\bar{x} \in M^{n}$

$$
M \vDash R[\bar{x}] \Leftrightarrow N \vDash R[f(\bar{x})] .
$$

c) f é uma $L$-imersão elementar se para toda fórmula $\varphi\left(v_{1}, \ldots, v_{n}\right)$ em $L$ e $\bar{x} \in M^{n}$,

$$
M \vDash \varphi[\bar{x}] \quad \Leftrightarrow \quad N \vDash \varphi[f(\bar{x})] .
$$

Quando o contexto permitir, o nome de $L$ será omitido da notação. L-estruturas e L-morfismos constituem uma categoria, indicada por $\mathbf{L}-\mathbf{m o d}$.

\footnotetext{
${ }^{6} \subseteq_{f}$ indica subconjunto finito.
} 


\section{Observação 2.15 .}

(1) Como o símbolo para igualdade está em $\operatorname{rel}(2), L$-imersões são injetoras.

(2) Se $f$ é um $L$-morfismo e $\tau\left(v_{1}, \ldots, v_{n}\right)$ é um termo em $L$, então

$$
\text { Para todo } \bar{x} \in M^{n}, \quad f\left(\tau^{M}(\bar{x})\right)=\tau^{N}(f(\bar{x})) .
$$

(3) $\operatorname{Sejam} \varphi \equiv R\left(\tau_{1}\left(v_{1}, \ldots, v_{n}\right), \ldots, \tau_{m}\left(v_{1}, \ldots, v_{n}\right)\right)$ uma fórmula atômica em $L$ e $f$ uma função verificando $(i)$ e (ii) em 2.14.(a). Então, $f$ é um $L$-morfismo sse

$$
\text { Para todo } \bar{x} \in M^{n}, \quad M \models \varphi[\bar{x}] \quad \Rightarrow \quad N \vDash \varphi[f(\bar{x})] .
$$

Além disso, $f$ é uma $L$-imersão sse

$$
\text { Para todo } \bar{x} \in M^{n}, \quad M \models \varphi[\bar{x}] \quad \Leftrightarrow \quad N \models \varphi[f(\bar{x})] \text {. }
$$

Lema 2.16. Seja $L$ uma linguagem de primeira ordem com igualdade e $f: M \longrightarrow N$ um L-morfismo.

a) $\operatorname{Se} \varphi\left(v_{1}, \ldots, v_{n}\right) \in \exists^{+}(L)$ e $\bar{a} \in M^{n}$, então

$$
M \vDash \varphi[\bar{a}] \Rightarrow N \vDash \varphi[f \bar{a}] .
$$

b) Se fé uma L-imersão e $\varphi\left(v_{1}, \ldots, v_{n}\right)$ é uma fórmula existencial em L, então

$$
M \vDash \varphi[\bar{a}] \Rightarrow N \vDash \varphi[f \bar{a}] \text {. }
$$

Prova. (a) e (b) seguem, por indução na complexidade, a partir de 2.15.(3).

Os conceitos e resultados que seguem são devidos a A. Robinson.

2.17. Os Diagramas de Robinson de uma $L$-estrutura : Seja $M$ uma $L$-estutura. Construímos uma nova linguagem, indicada por $L_{M}$, adicionando a $L$ um conjunto $\{a \underline{a}: a \in M\}$ de novas constantes. $M$ torna-se uma $L_{M}$-estrutura de forma natural da forma seguinte :

$$
\forall a \in M, \underline{a}^{M}=a \text {. }
$$

Definição 2.18. Seja $M$ uma L-estrutura.

a) O diagrama positivo de $M$ é definido como

$$
\Delta^{+}(M)=\left\{\varphi \in \operatorname{Sent}\left(L_{M}\right): \varphi \text { é atômica e } M \models \varphi\right\} \text {. }
$$

b) O diagrama de $M$ é definido como

$$
\Delta(M)=\left\{\varphi \in \operatorname{Sent}\left(L_{M}\right): \varphi \text { é atômica ou negação de atômica } \varepsilon \quad M \models \varphi\right\} \text {. }
$$

c) O diagrama completo de $M$ é definido como 


$$
\mathcal{T}(M)=\left\{\sigma \in \operatorname{Sent}\left(L_{M}\right): M \models \sigma\right\}
$$

Teorema 2.19. Sejam $M, N$ L-estruturas.

a) São equivalentes :

(1) Existe um um L-morfismo de $M$ em $N$;

(2) Podemos fazer de $N$ uma $L_{M}$-estrutura de modo que $N \models \Delta^{+}(M)$.

b) São equivalentes:

(1) Existe uma L-imersão de $M$ em $N$;

(2) Podemos fazer de $N$ uma $L_{M}$-estrutura de modo que $N \models \Delta(M)$.

c) São equivalentes:

(1) Existe uma L-imersão elementar de $M$ em $N$;

(2) Podemos fazer de $N$ uma $L_{M}$-estrutura de modo que $N \models \mathcal{T}(M)$.

Prova. (Esboço) Em todos os casos, a implicação $(2) \Rightarrow(1)$ é obtida mostrando que a função

$$
a \in M \longmapsto \underline{a}^{N} \in N
$$

tem as propriedades desejadas. Isto também indica como fazer de $N$ uma $L_{M}$-estrutura para provar que $(1) \Rightarrow(2)$. Para mais detalhes, veja Proposições 2.1 .12 (para (a)), 2.1.8 (para (b)) e 3.1 .3 (para (c)) em [CK].

2.20. Produtos em L-mod : Sejam $I$ um conjunto não vazio e $\left\{M_{i}: i \in I\right\}$, uma família de $L$-estruturas. Consideremos $M=\prod_{i \in I} M_{i}$ o produto dos conjuntos subjacentes. Fazemos de $M$ uma $L$-estrutura, definindo as interpretaçôes dos símbolos de $L$ coordenada a coordenada. Em mais detalhe, para cada natural $n \geq 1$ :

* Se $c \in c t, c^{M}=\left\langle c^{M_{i}}\right\rangle_{i \in I} ;$

* Se $\omega \in o p(n)$ e $\left\langle s_{1}, \ldots, s_{n}\right\rangle \in M^{n}$, então

$$
\omega^{M}\left(s_{1}, \ldots, s_{n}\right)=\left\langle\omega^{M_{i}}\left(s_{1}(i), s_{2}(i), \ldots, s_{n}(i)\right)\right\rangle_{i \in I} ;
$$

* Se $R \in \operatorname{rel}(n)$ e $\vec{s} \in M^{n}$, então

$$
M \vDash R[\bar{s}] \quad \Leftrightarrow \quad \forall i \in I, \quad M_{i} \models R\left[s_{1}(i), s_{2}(i), \ldots, s_{n}(i)\right] .
$$

Indução na complexidade fornece :

a) Se $\tau\left(v_{1}, \ldots, v_{n}\right)$ é um termo em $L$ e $\bar{s} \in M^{n}$, então

$$
\tau^{M}(\bar{s})=\left\langle\tau^{M_{i}}\left(s_{1}(i), \ldots, s_{n}(i)\right)\right\rangle_{i \in I} .
$$

b) Se $\varphi\left(v_{1}, \ldots, v_{n}\right)$ é uma fórmula atômica en $L$ e $\bar{x} \in M^{n}$, então

$$
M \models \varphi[\bar{s}] \quad \Leftrightarrow \quad \forall i \in I, M_{i} \models \varphi\left[s_{1}(i), \ldots, s_{n}(i)\right] .
$$


Observe que as projeções canônicas, $\pi_{i}: M \longrightarrow M_{i}$, são $L$-morfismos. É fácil ver que esta construção é o produto dos $M_{i}$ na categoria L-mod.

No caso em que $I=\emptyset$ temos o

Objeto final de $\mathbf{L}$-mod : ${ }^{7}$ Seja $\mathbb{I}=\{\emptyset\}$, onde todas as relações $n$-árias (distintas da igualdade) $\overline{\text { são interpretadas por } \mathbb{1}^{n}}$, todos os símbolos funcionais $n$-ários são interpretados pela projeção na primeira coordenada e todos os símbolos de constantes são interpretados pelo único elemento de $\mathbb{1 1}$. Vemos, por indução na complexidade, que toda $L$-fórmula positiva ${ }^{8}$ é satisfeita em $\mathbb{1 1}$; assim toda $L$-sentença da forma $\left.\forall \vec{x}\left(\psi_{0}(\vec{x}) \rightarrow \psi_{1}(\vec{x})\right)\right)$ onde $\psi_{0}(\vec{x}), \psi_{1}(\vec{x})$ são $L$-fórmulas positivas é verdadeira em $\boldsymbol{\pi}$.

Notamos ainda que $\prod_{i \in I} M_{i}=\emptyset$ sse existe $i \in I$ tal que $M_{i}=\emptyset .9$

\subsection{Sobre a estrutura vazia :}

a) Naturalmente podemos considerar estruturas vazias $(\emptyset)$ em linguagens $L$ tais que $\operatorname{ct}(L)=\emptyset$. Por exemplo, a linguagem $L_{O}$, adequada para tratar de conjuntos ordenados, contém apenas símbolos relacionais: $\operatorname{rel}\left(2, L_{O}\right)=\{=, \leq\}$. A importância do ordinal $\mathbf{0}$, o conjunto ordenado vazio, para a Matemática dispensa comentários.

b) A relação de satisfazibilidade $(\models)$ entre estruturas e fórmulas mediante a atribuição de valores para cada variável livre da fórmula em questão nos diz que: ${ }^{10}$

* Nenhuma fórmula aberta é satisfazível em $\emptyset$ (em particular a fórmula $\left.v_{0}=v_{0}\right)$;

* Nenhuma fórmula em $\Sigma_{n}, \mathrm{n} \geqslant 0$, é verdadeira em $\emptyset$ (em particular a fórmula $\exists v_{0}\left(v_{0}=v_{0}\right)$ );

* Toda fórmula em $\boldsymbol{\Pi}_{n}, \mathrm{n} \geqslant 0$, é verdadeira em $\emptyset$ (em particular a fórmula $\forall v_{0}\left(v_{0} \neq v_{0}\right)$ ).

Em particular vemos que a noção de "ser vazio" é axiomatizável pela sentença $\forall v_{0}\left(v_{0} \neq v_{0}\right)$.

c) Acreditamos que a versão adequada do axioma de instanciação, 11.a em 2.5, seja

$$
\text { 11.a' } \forall v \varphi \wedge \exists v_{0}\left(v_{0}=v_{0}\right) \rightarrow \varphi(\ulcorner\tau \mid v\urcorner)
$$

onde $\tau$ é termo livre para $v \operatorname{em} \varphi .{ }^{11}$

2.22. Produtos Reduzidos e Ultraprodutos em L-mod : Sejam $I$ um conjunto não vazio e $\left\{M_{i}: i \in I\right\}$ uma família de $L$-estruturas, todas não vazias. Fixemos $F$ um filtro em $I$ e consideremos $M=\prod_{i \in I} M_{i}^{12}$ o produto dos conjuntos subjacentes. Definimos uma relação $\theta_{F}$ em $M$ por

$$
x \theta_{F} y \quad \Leftrightarrow \quad\{i \in I: x(i)=y(i)\} \in F .
$$

\footnotetext{
${ }^{7} \mathrm{O}$ produto do diagrama vazio!

${ }^{8}$ Veja 2.4.(e).

${ }^{9}$ Uma versão do axioma da escolha.

${ }^{10} \mathrm{Veja}$ 2.4.(e) .

${ }^{11}$ Como em 2.4.(d).

${ }^{12} M \neq \emptyset$.
} 
É fácil verificar que $\theta_{F}$ é uma relação de equivalência em $M$. Indicaremos por

$$
M / F=\{x / F: x \in M\}^{13}
$$

o conjunto das classes de equivalência de $\theta_{F}$. Se $\bar{x} \in M^{n}$, definimos

$$
\bar{x} / F=\left\langle x_{1} / F, \ldots, x_{n} / F\right\rangle \in(M / F)^{n} .
$$

Para $\bar{x} \in M^{n}$ e $i \in I$, definimos

$$
\bar{x}(i)=\left\langle x_{1}(i), \ldots, x_{n}(i)\right\rangle \in M_{i}^{n} .
$$

Com a notação em 2.20 , se $R \in \operatorname{rel}(n, L), \omega \in o p(n, L)$ e $\bar{x}, \bar{y} \in M^{n}$ são tais que $\bar{x} / F=\bar{y} / F$, temos:

(1) $\omega^{M}(\bar{x}) / F=\omega^{M}(\bar{y}) / F$;

(2) $\left\{i \in I: M_{i} \vDash R[\bar{x}(i)]\right\} \in F \quad \Leftrightarrow \quad\left\{i \in I: M_{i} \models R[\bar{y}(i)]\right\} \in F$.

De posse de (1) e (2) fazemos de $M / F$ uma $L$-estrutura através das seguintes cláusulas :

* Se $c \in c t(L), \quad c^{M / F}=\left\langle c^{M_{i}}\right\rangle / F$, isto é, a interpretação da constante $c$ em $M / F$ é a classe da $I$-sequência cujas coordenadas são as interpretações de $c$ em cada $M_{i}$;

* Se $\omega \in o p(n, L)$ e $\bar{x} \in M^{n}, \omega^{M / F}(\bar{x} / F)=\omega^{M}(\bar{x}) / F$

* Se $R \in \operatorname{rel}(n, L)$ e $\bar{x} \in M^{n}$,

$$
M / F \models R[\bar{x} / F] \quad \Leftrightarrow \quad\left\{i \in I: M_{i} \vDash R[\bar{x}(i)]\right\} \in F .
$$

Por indução na complexidade obtemos

(3) Se $\tau\left(v_{1}, \ldots, v_{n}\right)$ é um termo em $L$ e $\bar{x} \in M^{n}, \tau^{M / F}(\bar{x} / F)=\tau^{M}(\bar{x}) / F$.

(4) Se $\varphi\left(v_{1}, \ldots, v_{n}\right)$ é uma fórmula atômica em $L$ e $\bar{x} \in M^{n}$,

$$
M / F \models \varphi[\bar{x} / F] \quad \Leftrightarrow \quad\left\{i \in I: M_{i} \models \varphi[\bar{x}(i)]\right\} \in F .
$$

(5) A aplicação natural $x \in M \longmapsto x / F \in M / F$ é um $L$-morfismo sobrejetor.

A $L$-estrutura $M / F$ denomina-se produto reduzido da família $\left\{M_{i}: i \in I\right\}$ pelo filtro $F$.

Se $F$ é um ultrafiltro em $I, M / F$ é o ultraproduto dos $M_{i}$ pelo filtro $F$. A notação usual para produtos reduzidos é

$$
\prod_{i \in I} M_{i} / F
$$

No caso em que todas as $L$-estruturas são as mesmas, $M_{i}=N, i \in I$, a construção correspondente denomina-se potência reduzida (ou ultrapotência, caso $F$ seja ultrafiltro), sendo indicada por $N^{I} / F$.

O resultado básico acerca de ultraprodutos é o seguinte

Teorema 2.23. (Lós) $S e\left\{M_{i}: i \in I\right\}$ é uma família de L-estruturas todas não vazias, $M=$ $\prod_{i \in I} M_{i}$ e $F$ é um ultrafiltro em $I \neq \emptyset$, para toda fórmula $\varphi\left(v_{1}, \ldots, v_{n}\right)$ em $L$ e todo $\bar{x} \in M^{n}$

${ }^{13} M / F \neq \emptyset$. 


$$
M / F \vDash \varphi[\bar{x} / F] \quad \Leftrightarrow \quad\left\{i \in I: M_{i} \vDash \varphi[\bar{x}(i)]\right\} \quad \in \quad F .
$$

Prova. Veja Teorema 4.1 .9 (pag. 217) em [CK] ou Teorema 5.2 .1 (pag. 90) em [BS].

Acrescentamos que a equivalência acima é verdadeira para produtos reduzidos em geral ( $F$ é filtro) se nos restringimos a fórmulas $\varphi\left(v_{1}, \ldots, v_{n}\right)$ que são existenciais positivas.

Se $M$ é um $L$-estrutura, $I$ é um conjunto e $F$ é um filtro em $I$, há um $L$-morfismo canônico, denominado diagonal, de $M$ em $M^{I} / F$

$$
\delta: M \longrightarrow M^{I} / F \text { dado por } \delta(a)=\langle a\rangle / F,
$$

que leva $a \in M$ na classe da $I$-sequência constante de valor $a$. Do Teorema 2.23 obtemos

Corolário 2.24. Se $U$ é um ultrafiltro em I então o L-morfismo diagonal, $\delta: M \longrightarrow M^{I} / U$, é uma L-imersão elementar.

Será importante mencionar os seguintes resultados fundamentais sobre ultrapotências.

Teorema 2.25. (D. S. Scott) Se $f: M \longrightarrow N$ é um L-morfismo, são equivalentes:

(1) f é uma L-imersão elementar;

(2) Existem um conjunto $I$, um ultrafiltro $U$ em I e uma L-imersão elementar $g: N \longrightarrow M^{I} / U$ tais que o seguinte diagrama é comutativo

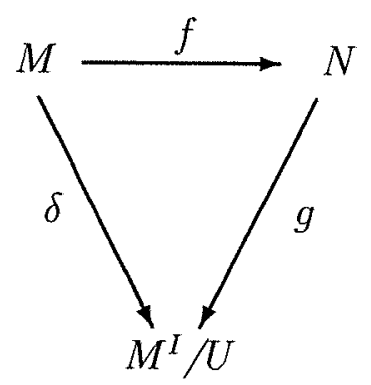

onde $\delta$ é o L-morfismo diagonal.

Prova. Veja Lema 8.1 .3 em [BS].

Teorema 2.26.(Kiesler-Shelah) Duas L-estruturas são elementarmente equivalentes sse têm ultrapotências isomorfas. 
Prova. Veja Teorema 6.1 .15 (pag. 398) em [CK] ou Teoremas 7.2.5 e 7.2 .6 (pag. 150) em $[\mathrm{BS}]^{14}$.

\section{$2.3 \quad$ Limites em L-mod}

Teorema 2.27. Seja L uma linguagem de primeira ordem com igualdade.

a) A categoria L-mod é completa.

b) Sejam $\mathcal{I}$ uma categoria pequena, $D$ um $\mathcal{I}$-diagrama em L-mod e lim $D$ o seu limite. Seja $\sigma$ uma sentença em $L$, logicamente equivalente à uma conjunção finita $\overleftarrow{d e}$ sentenças da forma $\forall \bar{x}(\forall \bar{y} \psi \rightarrow \varphi)$, onde $\psi$ e $\varphi$ são positivas e livre de quantificadores. ${ }^{15}$ Então,

$$
\forall i \in \operatorname{Obj}(\mathcal{I}), \quad D(i) \models \sigma \quad \Rightarrow \quad \lim D \models \sigma .
$$

\section{Prova.}

a) Utilizaremos o Teorema 1.47, verificando que L-mod tem produtos e equalizadores. Por 2.20, L-mod possui produtos de qualquer família de objetos.

Equalizadores em L-mod : Sejam $D=(A \underset{g}{\stackrel{f}{\longrightarrow}} B) L$-morfismos. Definimos

$$
E=\{a \in A: f(a)=g(a)\} .
$$

Se $c \in c t(L), c^{E}=_{\text {def }} c^{A} \in E$. Além disso, se $\omega \in o p(n, L)$ e $\bar{a} \in E^{n}$, temos

$$
f\left(\omega^{A}(\bar{a})\right)=\omega^{B}(f(\bar{a}))=\omega^{B}(g(\bar{a}))=g\left(\omega^{A}(\bar{a})\right),
$$

e $E$ é fechado em relação à interpretação dos símbolos funcionais. Para $R \in \operatorname{rel}(n, L)$, seja $R^{E}=R \cap E^{n}$. Então, a imersão canônica, $\eta: E \rightarrow A$, é uma $L$-imersão. Além disso, como $f \circ \eta=g \circ \eta,(E ;\{\eta, f \circ \eta\})$ é um cone sobre $D$ em L-mod. Mostraremos que este cone é o equalizador de $(f, g)$.

Seja $h: C \longrightarrow A$ um $L$-morfismo, tal que $g \circ h=f \circ h$. Note que esta equação implica que para todo $x \in C, h(x) \in E$. Definimos $\beta: C \longrightarrow E$ por $x \mapsto h(x)$. Está claro que $\beta$ é a única função tal que $h=\eta \circ \beta$. É fácil verificar que $\beta$ é uma $L$-morfismo, como necessário.

\footnotetext{
${ }^{14}$ Nessa apresentação a prova depende da Hipótese Generalizada do Contínuo (HGC).

${ }^{15}$ Veja 2.4.(e).
} 


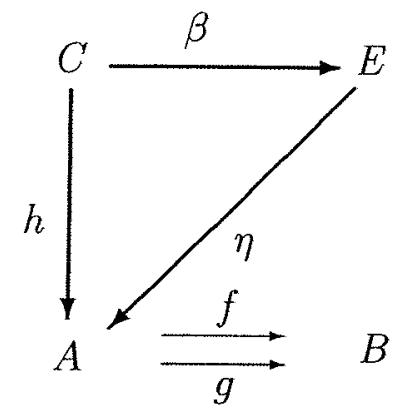

b) Faremos um esboço da prova, omitindo detalhes.

Sejam

$$
D: \mathcal{I} \longrightarrow \mathbf{L}-\bmod :(i \stackrel{\alpha}{\longrightarrow} j) \mapsto(D(i) \stackrel{D(\alpha)}{\longrightarrow} D(j))
$$

um $\mathcal{I}$-diagrama em $\mathbf{L}$-mod e

um cone limite de $D$.

$$
\left(M \stackrel{\lambda_{i}}{\longrightarrow} D(i): i \in \operatorname{Obj}(\mathcal{I})\right)=\underset{\leftarrow}{\lim } D
$$

Sejam $\Sigma$ e $\Pi$ os conjuntos de fórmulas $\varphi\left(v_{1}, \ldots, v_{n}\right)$ em $L$ definidos pelas seguintes clausulas :

$$
\begin{aligned}
& \varphi \in \Sigma \quad \Leftrightarrow \quad \forall \bar{s} \in M^{n}, \quad\left(M \models \varphi[\bar{s}] \Leftrightarrow D(i) \models \varphi\left[\lambda_{i}(\bar{s})\right]\right) ; \\
& \varphi \in \Pi \quad \Leftrightarrow \quad \forall \bar{s} \in M^{n},\left(D(i) \models \varphi\left[\lambda_{i}(\bar{s})\right] \Rightarrow M \models \varphi[\bar{s}]\right) .
\end{aligned}
$$

Então pode-se verificar que :

* Todas as fórmulas atômicas estão em $\Sigma$;

$* \psi, \varphi \in \Sigma \Rightarrow \psi \wedge \varphi \in \Sigma$;

$* \psi \in \Pi, \varphi \in \Sigma \Rightarrow \forall x \psi \in \Pi$ e $\psi \rightarrow \varphi \in \Pi$.

Está claro que o resultado desejado segue dos enunciados acima.

A seguinte caracterização conjuntística de limites de $\mathcal{I}$-diagramas é útil :

Corolário 2.28. Sejam $D: \mathcal{I} \longrightarrow \mathbf{L}-\bmod :(i \stackrel{\alpha}{\longrightarrow} j) \mapsto(D(i) \stackrel{D(\alpha)}{\longrightarrow} D(j))$ um $\mathcal{I}$ diagrama em $\mathbf{L}-\bmod e\left(M \stackrel{\lambda_{i}}{\rightarrow} D(i): i \in \operatorname{Obj}(\mathcal{I})\right)$ um cone sobre $D$. Consideremos $\lambda=$ $\left(\lambda_{i}\right)_{i \in I}$ a única função de $M$ para $\prod D(i)$, tal que $\pi_{i} \circ \lambda=\lambda_{i}$, para cada $i \in \operatorname{Obj}(\mathcal{I})$. Então, $M$ é (isomorfo a) $\lim _{\leftarrow} D$ sse

[lim 1] : A imagem de $\lambda$ em $\prod D(i)$ é o conjunto

$$
\left\{x \in \prod D(i): \text { para toda flecha de } \mathcal{I},(i \stackrel{\alpha}{\longrightarrow} j) \text {, temos } f_{\alpha}\left(\pi_{i}(x)\right)=\pi_{j}(x)\right\} .
$$

[lim 2] : Se $\varphi\left(v_{1}, \ldots, v_{n}\right)$ é uma fórmula atômica em $L$ e $\bar{s} \in M^{n}$,

$$
M \vDash \varphi[\bar{s}] \Leftrightarrow \forall i \in \operatorname{Obj}(\mathcal{I}), D(i) \vDash \varphi\left[\lambda_{i}(\bar{s})\right] .
$$


Agora discutiremos limites para morfismos de $\mathcal{I}$-diagramas em L-mod.O contexto geral para este assunto foi exposto na seção 1.2.5 do Capítulo anterior.

Explicitamos abaixo a definição da categoria $\operatorname{Diag}_{\Omega}(\mathbf{L}-\mathbf{m o d})$ (ver definição 1.52), onde $\Omega$ é a subcategoria de Cat com um único objeto $\mathcal{I}$ e $\overleftarrow{u m a}$ única flecha $I d_{\mathcal{I}}: \mathcal{I} \longrightarrow \mathcal{I}$. As proposição que se segue é a 1.54 , no contexto aqui considerado.

Definição 2.29. Sejam $\langle I, \leq\rangle$ um conjunto parcialmente ordenado $e$

$$
D=\left(D(i) ;\left\{f_{i j}: i \leq j\right\}\right) \text { e } E=\left(E(i) ;\left\{g_{i j}: i \leq j\right\}\right)
$$

I-diagramas em L-mod. Um morfismo, $h: D \longrightarrow E$, consiste de uma família de L-morfismos, $h_{i}: D(i) \longrightarrow E(i), i \in I$, tal que para $i \leq j$ em $I$, o seguinte diagrama é comutativo :

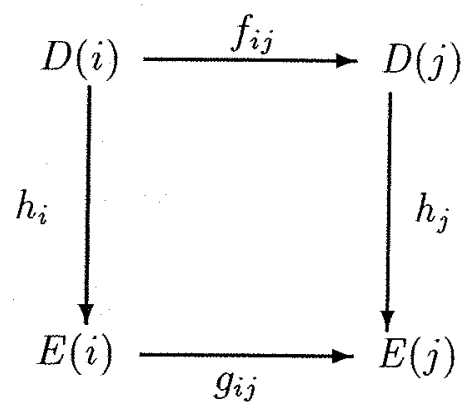

Está claro que $I d_{D}=\left(I d_{D(i)}\right)_{i \in I}$ é um morfismo; se $D \stackrel{h}{\longrightarrow} E \stackrel{k}{\longrightarrow} G$ são morfismos de $I$ diagramas, então

$$
k \circ h=\left(k_{i} \circ h_{i}\right)_{i \in I}
$$

é um morfismo de I-diagramas.

Proposição 2.30. Seja $h: D \longrightarrow E$ um morfismo de I-diagramas em L-mod. Então existe um único L-morfismo

$$
\stackrel{\lim }{\leftarrow}: \lim D \longrightarrow \lim E
$$

tal que para todo $i \in I$, o seguinte diagrama é comutativo:

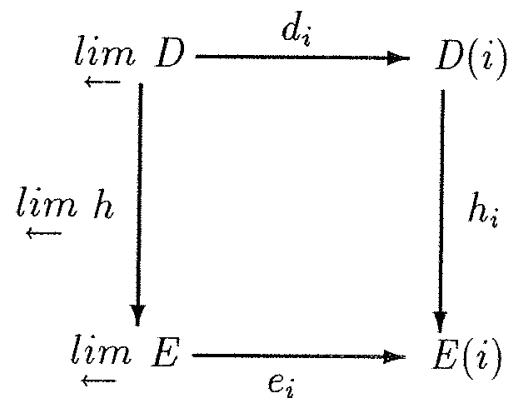

onde $d_{i}$ e $e_{i}$ são os L-morfismos canônicos que acompanham os limites de $D$ e E. 
Prova. Está claro que $\left\langle\lim D ;\left\{h_{i} \circ d_{i}: i \in I\right\}\right\rangle$ é um cone sobre $E$; a propriedade universal dos limites garante a existência e unicidade de $\lim h$.

\subsection{Quocientes em L-mod}

Definição 2.31. Seja A uma L-estrutura. Uma relação de equivalência $\theta$ em A é uma $\boldsymbol{L}$ congruência se for uma congruência com respeito a todas as $L$-operaçốes, isto é, se $\omega \in$ op $(n, L)$ e $x_{1}, \ldots, x_{n}, y_{1}, \ldots, y_{n} \in A^{n}$ então

$$
x_{j} \theta y_{j}, 1 \leq j \leq n \quad \Rightarrow \quad \omega^{M}\left(x_{1}, \ldots, x_{n}\right) \theta \omega^{M}\left(y_{1}, \ldots, y_{n}\right) .
$$

Lema 2.32. Se $f: M \longrightarrow N$ é um L-morfismo, definimos

$$
\theta_{f}=\left\{\langle x, y\rangle \in M^{2}: f(x)=f(y)\right\} .
$$

Então, $\theta_{f}$ é uma L-congruência em $M$.

Prova. Suponha que $\omega \in o p(n, L)$ e que $x_{1}, \ldots, x_{n}, y_{1}, \ldots, y_{n} \in M^{n}$ são tais que $x_{j} \theta_{f} y_{j}$, $1 \leq j \leq n$. Então, do fato que $f$ é um $L$-morfismo vem

$$
f\left(\omega^{M}(\bar{x})\right)=\omega^{N}(f(\bar{x}))=\omega^{N}(f(\bar{y}))=f\left(\omega^{M}(\bar{y})\right)
$$

mostrando que $\omega^{M}(\bar{x}) \theta_{f} \omega^{M}(\bar{y})$.

Proposição 2.33. Seja A uma L-estrutura e $\theta$ um L-congruência em A. Então, o conjunto $A / \theta$ de classes de equivalência de $A$ por $\theta$ torna-se uma $L$-estrutura da seguinte forma :

(1) Para $R \in \operatorname{rel}(n, L)$ e $\bar{x} \in A^{n}$,

$$
A / \theta=R[\bar{x} / \theta] \quad \Leftrightarrow \quad\left\{\begin{array}{c}
\exists \bar{y} \in A^{n} \text { tal que } y_{k} \theta x_{k}, 1 \leq k \leq n \\
\text { e } A \models R[\bar{y}]
\end{array}\right.
$$

onde $\bar{x} / \theta=\left\langle x_{1} / \theta, \ldots, x_{n} / \theta\right\rangle$.

(2) Para $\omega \in \operatorname{op}(n, L)$ e $\bar{x} \in A^{n}, \quad \omega^{A / \theta}(\bar{x})=\omega^{A}(\bar{x}) / \theta$.

(3) Para $c \in \operatorname{ct}(L), c^{A / \theta}=c^{A} / \theta$.

Com esta L-estrutura, a função quociente canônica, $\pi_{\theta}: A \longrightarrow A / \theta$ é um L-morfismo. Além disso, se $f: A \longrightarrow M$ é um L-morfismo e $\theta \subseteq \theta_{f}$ (2.32), existe um único L-morfismo, $\widehat{f}: A / \theta \longrightarrow M$, tal que o seguinte diagrama é comutativo : 


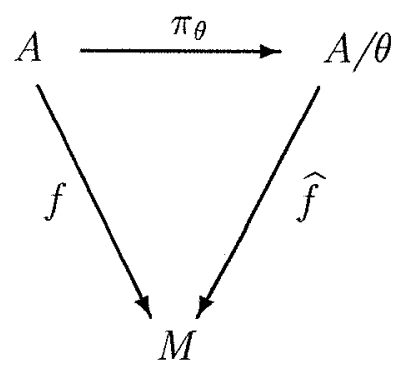

Prova. As aplicações em (2) estão bem definidas pois $\theta$ é uma congruência em relação à todas as operações em $L$. Além disso, (2) implica que $\pi_{\theta}$ preserva todas as $L$-operações em $A$, enquanto que (3) acarreta que a interpretação das constantes também é preservada. Por fim, a condição (1) garante que a interpretação das relações são preservadas e portanto $\pi_{\theta}$ é um $L$-morfismo.

Se $M$ é uma $L$-estrutura e $f: A \longrightarrow M$ é uma $L$-morfismo, definimos, para $x \in A$,

$$
\widehat{f}(x / \theta)=f(x) \text {. }
$$

Como $\theta \subseteq \theta_{f}(2.32), \widehat{f}$ está bem definido. Se $R \in \operatorname{rel}(n, L)$, suponha que $A / \theta \neq R[\bar{x} / \theta]$. Então existe $\bar{y} \in A^{n}$ tal que $A \models R[\bar{y}]$ e $x_{k} \theta y_{k}, 1 \leq k \leq n$. Como $f$ é um $L$-morphism, concluímos que

$$
M \models R[f(\bar{y})] .
$$

Mas $x_{k} \theta y_{k}$ e $\theta \subseteq \theta_{f}$ implicam que $f\left(x_{k}\right)=f\left(y_{k}\right), 1 \leq k \leq n$, e assim

$$
M \vDash R[\widehat{f}(\bar{x} / \theta)]
$$

como necessário. Está claro que para toda constante $c$ em $L, \widehat{f}\left(c^{A / \theta}\right)=c^{M}$. Para símbolos operacionais o argumento é análogo. A unicidade de $f$ é imediata.

A $L$-estrutura $A / \theta$ definida em 2.33 é o quociente de $A$ pela congruência $\theta$.

Evidentemente o conjunto das congruências sobre um $L$-estrutura $A$ é um conjunto fechado por interseções arbitrárias, portanto a todo subconjunto $X \subseteq A \times A$ podemos associar $\theta_{X}$, a congruência gerada por $X$, que é a menor congruência em $A$ que contém $X$.

\subsection{Colimites em L-mod}

Mencionamos que a categoria L-mod é cocompleta. Uma prova pode ser feita utilizando a versão dual do Teorema 1.47, i.e., verificando que L-mod possui coequalizadores e coprodutos. Coequalizadores de pares paralelos de $L$-morfismos $(M \underset{g}{\rightrightarrows} N)$ são construídos considerando-se o $L$-morfismo quociente $\pi_{\theta}: N \longrightarrow N / \theta$, onde $\theta$ é a congruência em $N$ gerada pelo conjunto $X(f, g)=\{\langle f(m), g(m)\rangle: m \in M\} \subseteq N \times N$. A construção de coprodutos é um pouco mais técnica e não será necessária neste trabalho.

No resto da seção estudamos um tipo particular de colimite em $\mathbf{L}_{-m} \bmod$ que será muito útill. 
Teorema 2.34. Sejam $\langle I, \leq\rangle$ um conjunto parcialmente ordenado e dirigido e L uma linguagem de primeira ordem com igualdade.

a) Todo I-diagrama em L-mod tem colimite.

b) Se $D$ é um I-diagrama em $\mathbf{L}$-mod e $\sigma$ é uma sentença em L, logicamente equivalente a uma conjunção finita de sentenças da forma $\forall \bar{x}(\psi \rightarrow \exists \bar{y} \varphi)$, onde $\psi$ e $\varphi$ são positivas e livre de quantificadores ${ }^{16}$, então

$$
\forall i \in I, D(i) \vDash \sigma \quad \Rightarrow \quad \lim D \vDash \sigma
$$

\section{Prova.}

a) Seja $A=\coprod_{i \in I} D(i)=\bigcup_{i \in I} D(i) \times\{i\}$ a união disjunta dos $D(i)$. Temos funções canônicas, $w_{i}: D(i) \longrightarrow A, x \mapsto\langle x, i\rangle$. Como $I$ é dirigido, a prescrição

$$
\langle x, i\rangle \equiv\langle y, j\rangle \Leftrightarrow \exists k \geq i, j \text { tal que } f_{i k}(x)=f_{j k}(y)
$$

define uma relação de equivalência $\equiv$ em $A$. Seja

$$
G=\{\langle x, i\rangle / \equiv:\langle x, i\rangle \in A\}
$$

o conjunto das classes de equivalência de $\equiv$. Note que para uma constante $c \operatorname{em} L$ temos $\left\langle c^{D(i)}, i\right\rangle$ $\equiv\left\langle c^{D(j)}, j\right\rangle$. Interpretamos $L$ em $G$ da seguinte forma : Para $n \geq 1$ e $\bar{x} \in G^{n}$,

$$
\bar{x}=\left\langle\left\langle x_{1}, i_{1}\right\rangle / \equiv, \ldots,\left\langle x_{n}, i_{n}\right\rangle / \equiv\right\rangle
$$

definimos

i) Se $R \in \operatorname{rel}(n, L)$; então $G \models R[\bar{x}]$ sse

$$
\exists k \geq i_{1}, \ldots, i_{n}, \text { tal que } D(k) \vDash R\left[f_{i_{1} k}\left(x_{1}\right), \ldots, f_{i_{n k}}\left(x_{n}\right)\right] .
$$

ii) Se $\omega \in o p(n, L)$, tomamos $k \geq i_{1}, \ldots, i_{n}$ e definimos $\omega^{G}(\bar{x})$ como a classe de equivalência do $\operatorname{par}\left\langle\omega^{D(k)}\left(f_{i_{1} k}\left(x_{1}\right), \ldots, f_{i_{n} k}\left(x_{n}\right)\right), k\right\rangle$.

iii) Se $c \in \operatorname{ct}(L), c^{G}=\left\langle c^{D(i)}, i\right\rangle / \equiv$.

Uma vez que $I$ é dirigido, as construções acima são independentes de representantes e da escolha de índices utilizada acima. Além disso, a composição da função quociente, $A \longrightarrow G$, com as funções $w_{i}$, define $L$-morfismos $\alpha_{i}$ de $D(i)$ para $G$, fazendo de $\left(G,\left\{\alpha_{i}: i \in I\right\}\right)$ um co-cone sobre $D$. Se $\left(N ;\left\{g_{i}: i \in I\right\}\right)$ é um co-cone sobre $D$, considere $h: G \longrightarrow N$ dada por $h(\langle x, i\rangle / \equiv)=$ $g_{i}(x)$ : é fácil verificar que $h$ é uma função bem definida, que é $L$-morfismo e é único $L$-morfismo tal que $h \circ \alpha_{i}=g_{i}, i \in I$. Portanto $\left.\left(G ;\left\{\alpha_{i}: i \in I\right\}\right)\right)$ é $\stackrel{\lim }{\rightarrow} D$. A prova de (b) pode ser obtida pelo mesmo método usado para limites em 2.27 .

Observação 2.35. Os Teoremas 2.27 e 2.34 implicam que subcategorias (plenas) de categorias L-mod como a dos grupos, dos anéis, das álgebras de Boole, ... e seus homomorfismos são fechadas por todos os limites de diagramas pequenos e por colimites de diagramas filtrantes.

Por um resultado de H. J. Kiesler, os itens (b) de 2.27 e 2.34 são os melhores possíveis, isto é,

\footnotetext{
${ }^{16}$ Veja 2.4.
} 
sentenças que não forem dos tipos indicados podem não ser preservadas por limites ou colimites dirigidos.

Proposição 2.36. Seja $D=\left\langle D(i) ;\left\{f_{i j}: i \leq j\right\}\right\rangle$ um I-diagrama em L-mod, onde $\langle I, \leq\rangle$ é parcialmente ordenado e dirigido. Seja $\left\langle M ;\left\{f_{i}: i \in I\right\}\right\rangle=\lim _{\rightarrow} D$. Então:

a) Se cada $f_{i j}$ é uma L-imersão, o mesmo é verdade para $f_{i}, i \in I$.

b) (Tarski) Se cada $f_{i j}$ é uma imersão elementar, o mesmo é verdade para $f_{i}, i \in I$.

Prova. O item (a) é imediato. Para (b), utilizamos indução na complexidade das fórmulas.

Os resultados correspondentes a 2.28 e 2.30 para colimites são os seguintes :

Corolário 2.37. Seja $D=\left(D(i),\left\{f_{i j}: i \leq j\right\}\right)$ um I-diagrama em L-mod, com I parcialmente ordenado e dirigido. Um co-cone em $\mathbf{L}$-mod sobre $D,\left\langle G ;\left\{\alpha_{i}\right\}_{i \in I}\right\rangle$, é (isomorfo a) $\lim _{\longrightarrow}$ $D$ sse verifica as seguintes condições :

$[\operatorname{colim} 1]: G=\bigcup\left\{\alpha_{i}(D(i)): i \in I\right\}$.

[colim 2] : Se $\varphi\left(v_{1}, \ldots, v_{n}\right)$ é uma fórmula atômica em $L$ e $\bar{s} \in G^{n}$,

$$
G \vDash \varphi[\bar{s}] \quad \Leftrightarrow \quad\left\{\begin{array}{c}
\exists k \in I \text { e } \bar{x} \in D(k)^{n} \text { tal que } \\
s_{p}=\alpha_{k}\left(x_{p}\right), 1 \leq p \leq n, \\
e D(k) \models \varphi[\bar{x}] .
\end{array}\right.
$$

A proposição abaixo é a dualização da proposição 2.30 .

Proposição 2.38. Sejam $\langle I, \leq\rangle$ um conjunto ordenado dirigido e $h: D \longrightarrow E$ um morfismo de I-diagramas em L-mod. Então existe um único L-morfismo

$$
\underset{\lim }{\rightarrow} h: \lim _{\rightarrow} D \longrightarrow \lim E
$$

tal que para todo $i \in I$ o seguinte diagrama é comutativo :

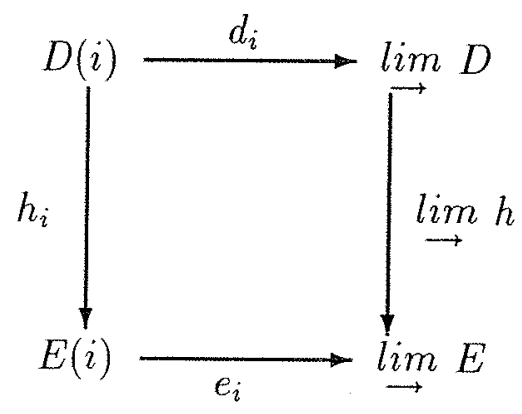


onde $d_{i}$ e $e_{i}$ são os L-morfismos que acompanham $\lim _{\rightarrow} D$ e $\lim _{\rightarrow} E$. Para $\chi \in \underset{\rightarrow}{\lim } D$, se $i \in I e$ $x \in D(i)$ são tais que $d_{i}(x)=\chi$, então

$$
\lim _{\longrightarrow} h(\chi)=e_{i}\left(h_{i}(x)\right) .
$$

Além disso, se cada $h_{i}$ é uma L-imersão, o mesmo é verdade para $\underset{\rightarrow}{\lim } h$.

Há uma ligação entre colimites filtrantes e produtos reduzidos que será útil na prova do Teorema 2.50. Antes do enunciado e da prova estabelecemos alguma notação.

2.39. Sejam $L$ uma linguagem de primeira ordem com igualdade, $I$ um conjunto não vazio, $\left\{M_{i}: i \in I\right\}$ uma família de $L$-estruturas não vazias e $M=\prod_{i \in I} M_{i}$ o seu produto (2.20).

(1) Para $J \subseteq I$, seja $M_{\mid J}=\prod_{j \in J} M_{j} ;{ }^{17}$

(2) Se $J \subseteq K \subseteq I$, há uma $L$-morfismo canônico, $\pi_{K J}: M_{\mid K} \longrightarrow M_{\mid J}$, que esquece as coordenadas fora de $J$, isto é, para $x \in M_{\mid K}, \pi_{K J}(x)=x_{\mid J}{ }^{18}$. Note que

$$
\pi_{J J}=I d_{M_{\mid J}} \quad \text { e } \quad J \subseteq K \subseteq W \Rightarrow \pi_{W J}=\pi_{K J} \circ \pi_{W K} .
$$

As projeções canônicas, $\pi_{i}: M \longrightarrow M_{i}$, correspondem à $\pi_{I\{i\}}$.

(3) Fixado $J \subseteq I$, definimos $*: M_{\mid J} \times M \longrightarrow M,\langle s, x\rangle \longmapsto s * x$, onde

$$
s * x(i)= \begin{cases}s(i) & \text { se } i \in J \\ x(i) & \text { se } i \notin J .\end{cases}
$$

Note que $J=I$, a operação * é a projeção na primeira coordenada. Equivalentemente, para cada $x \in M$, a função $(\cdot) * x: M \longrightarrow M$ é a identidade.

Seja $\mathcal{F}$ um filtro em $I$. Então, $\langle\mathcal{F}, \subseteq\rangle$ é um conjunto parcialmente ordenado e dirigido para baixo (1.1) pois se $J, K \in \mathcal{F}$, então $J \cap K \in \mathcal{F}$. Consequentemente, $\mathcal{F}^{o p}$, o conjunto parcialmente ordenado oposto de $\langle\mathcal{F}, \subseteq\rangle(1.2)$ é dirigido. Consideremos

$$
\mathcal{M}=\left(M_{\mid J} ; \quad\left\{\pi_{K J}: J \subseteq K \text { e } J \in \mathcal{F}\right\}\right) ;
$$

Por $\left(^{*}\right)$ em (2), $\mathcal{M}$ é $\mathcal{F}^{o p}$-diagrama em $\mathbf{L}$-mod, na realidade um sistema dirigido sobre $\mathcal{F}^{o p}$ em L-mod (1.41.(3)), o sistema dirigido associado à família $\left\{M_{i}: i \in I\right\}$ e ao filtro $\mathcal{F}$ em $I$.

Proposição 2.40. Sejam L uma linguagem de primeira ordem com igualdade, I um conjunto não vazio, $\left\{M_{i}: i \in I\right\}$ uma familia de L-estruturas não vazias e $\mathcal{F}$ um filtro em $I$. Seja

$$
\mathcal{M}=\left(M_{\mid J} ; \quad\left\{\pi_{K J}: J \subseteq K \quad \text { e } J \in \mathcal{F}\right\}\right)
$$

o sistema dirigido sobre $\mathcal{F}^{o p}$ associado à família $\left\{M_{i}: i \in I\right\}$ e ao filtro $\mathcal{F}$ com em 2.39. Então,

a) $\lim \mathcal{M}=\prod_{i \in I} M_{i} \cdot{ }^{19}$

\footnotetext{
${ }^{17}$ Em particular, $M_{\mid I}=M$.

${ }^{18}$ Lembre-se que $x$ é uma função de $K$ em $\bigcup_{k \in K} M_{k}$.

${ }^{19}$ Assim, o limite do $\mathcal{F}^{o p}$-diagrama $\mathcal{M}$ em L-mod é a $L$-estrutura produto $(2.20)$.
} 
b) $\underset{\lim }{\rightarrow} \mathcal{M}$ é naturalmente $L$-isomorfo ao produto reduzido $\prod_{i \in I} M_{i} / \mathcal{F}(2.22)$.

Prova. Adotamos a notação em 2.39. Em particular, $M=\prod_{i \in I} M_{i}$ é a $L$-estrutura produto. $\mathrm{O}$ item (a) é imediato, posto que $I \in \mathcal{F}$. Na realidade

$$
\lim _{\leftarrow} \mathcal{M}=\left(M ;\left\{\pi_{I J}: J \subseteq I\right\}\right) .
$$

Para (b), fixemos $t \in M$. Dado $J \subseteq I$, definimos $\nu_{J}: M_{\mid J} \longrightarrow M / \mathcal{F}$, dada por

$$
\nu_{J}(s)=(s * t) / \mathcal{F} \text {. }
$$

Fato 2.41. Sejam $J, K \subseteq I, \operatorname{com} J \in \mathcal{F}$.

a) A função $\nu_{J}$ indepedende do elemento $t$ fixado em $M$ e é um $L$-morfismo.

b) Se $J \subseteq K$, o seguinte diagrama é comutativo :

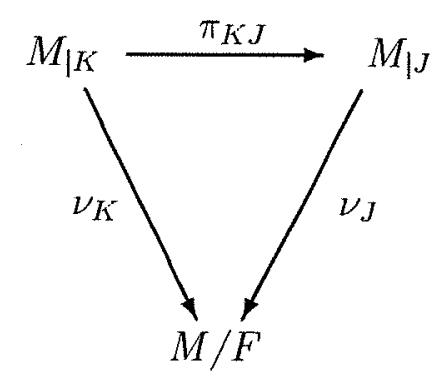

Prova.

a) Como $J \in \mathcal{F}$, se $x \in M$, então segue da definição da operação $*$ em 2.39.(3) que para todo $s$ $\in M_{\mid J}$

$$
J \subseteq\{i \in I: s * t(i)=s * x(i)\}
$$

e portanto este último conjunto está em $\mathcal{F}$. Assim,

$$
(s * t) / \mathcal{F}=(s * x) / \mathcal{F}
$$

como desejado. Faremos a verificação de que $\nu_{J}$ preserva as operações em $L$, omitindo os detalhes para símbolos relacionais e constantes. Se $\omega \in o p(n, L)$ e $\vec{s} \in M_{\mid J}^{n}$, então :

(i) $\nu_{J}\left(\omega^{M_{\mid J}}(\bar{s})\right)=\left(\omega^{M_{\mid J}}(\bar{s}) * t\right) / \mathcal{F}$

(ii) Por 2.22, $\omega^{M / \mathcal{F}}\left(\nu_{J}(\bar{s})\right)=\omega^{M / \mathcal{F}}\left(s_{1} * t / \mathcal{F}, \ldots, s_{n} * t / \mathcal{F}\right)=\omega^{M}\left(s_{1} * t, \ldots, s_{n} * t\right) / \mathcal{F}$.

Assim, para mostrar que $\nu_{J}$ preserva $\omega$ é suficiente verificar que

$$
\left(\omega^{M_{\mid J}}(\bar{s}) * t\right) / \mathcal{F}=\omega^{M}\left(s_{1} * t, \ldots, s_{n} * t\right) / \mathcal{F}
$$

Da definição da operação * em 2.39.(3), do fato que $\pi_{I J}$ é um $L$-morfismo, juntamente com $\pi_{I J}\left(s_{k} * t\right)=s_{k}, 1 \leq k \leq n$, obtemos

$$
\pi_{I J}\left(\omega^{M}\left(s_{1} * t, \ldots, s_{n} * t\right)\right)=\omega^{M_{I J}}(\bar{s})=\pi_{I J}\left(\omega^{M_{I J}}(\bar{s}) * t\right) .
$$

Logo, se 


$$
K=\left\{i \in I:\left(\omega^{M}\left(s_{1} * t, \ldots, s_{n} * t\right)\right)(i)=\left(\omega^{M_{\mid J}}(\bar{s}) * t\right)(i)\right\}
$$

então $J \subseteq K$, estabelecendo (I). O item (b) é imediato a partir das definições.

O Fato 2.41 garante que

$$
M / \mathcal{F}=\left(M / \mathcal{F} ;\left\{\nu_{J}: J \in \mathcal{F}\right\}\right)
$$

é um co-cone sobre o diagrama $\mathcal{M}$. Devemos mostrar que este co-cone é $\lim _{\rightarrow} \mathcal{M}$. Seja

$$
\Lambda=\left(\Lambda ;\left\{\lambda_{J}: J \in \mathcal{F}\right\}\right),
$$

um co-cone sobre $\mathcal{M}$. Como $M_{\mid I}=M$ temos que $\nu_{I}: M_{\mid I} \longrightarrow M / \mathcal{F}$ é um L-morfismo sobrejetor, portanto basta mostrarmos que existe algum morfismo de co-cones em $\mathbf{L}$-mod de $M / \mathcal{F}$ para $\Lambda$. Dado $x \in M$, definimos

$$
f(x / \mathcal{F})=\lambda_{I}(x)
$$

Suponha que $y \in M$ é tal que $x / \mathcal{F}=y / \mathcal{F}$, isto é,

$$
J=\{i \in I: x(i)=y(i)\} \in \mathcal{F} .
$$

Como o diagrama à esquerda em (II) é comutativo e $\pi_{I J}(x)=\pi_{I J}(y)$
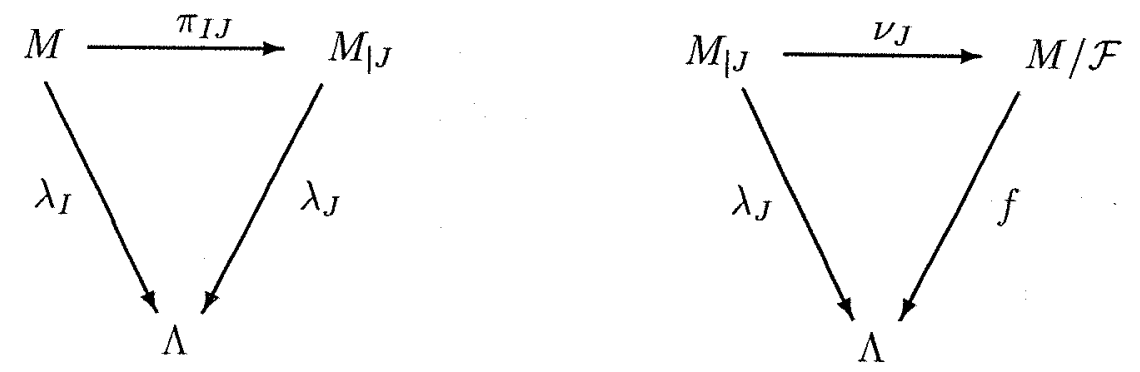

obtemos

$$
\lambda_{I}(y)=\lambda_{J}\left(\pi_{I J}(y)\right)=\lambda_{J}\left(\pi_{I J}(x)\right)=\lambda_{I}(x)
$$

mostrando que o valor de $f$ independe de representantes. Como toda classe em $M / \mathcal{F}$ é a classe de um elemento de $M$, está definida uma função

$$
f: M / \mathcal{F} \longrightarrow \Lambda, f(x / \mathcal{F})=\lambda_{I}(x) .
$$

Se $J \subseteq I$ e $s \in M_{\mid J}$ temos

$$
f\left(\nu_{J}(s)\right)=\lambda_{I}(s * t)=\lambda_{J}\left(\pi_{I J}(s * t)\right)=\lambda_{J}(s)
$$

pois $\pi_{I J}(s * t)=s$. Logo, para todo $J \subseteq I$ o diagrama á direita em (I) é comutativo. Para completar a prova, resta verificar que $f$ é um $L$-morfismo. Faremos isto para relações em $L$, omitindo os detalhes para símbolos funcionais e constantes.

Se $R \in \operatorname{rel}(n, L), \bar{x} \in M^{n}$ e $M / \mathcal{F} \vDash R[\bar{x} / \mathcal{F}]$, por 2.22.(4) obtemos

$$
J=\left\{i \in I: M_{i} \models R[\bar{x}(i)]\right\} \in \mathcal{F} .
$$

Por 2.20.(b), isto significa que $M_{\mid J} \vDash R\left[\pi_{I J}(\bar{x})\right]$. Uma vez que $\lambda_{J}: M_{\mid J} \longrightarrow \Lambda$ é um $L$-morfismo, obtemos 


$$
\Lambda \models R\left[\lambda_{J}\left(\pi_{I J}(\bar{x})\right)\right] .
$$

Agora, a definição de $f$ e a comutatividade do diagrama à esquerda em (II) fornecem

$$
f(\bar{x} / \mathcal{F})=\lambda_{I}(\bar{x})=\lambda_{J}\left(\pi_{I J}(\bar{x})\right)
$$

e $\Lambda \models R[f(\bar{x} / \mathcal{F})]$ é consequência imediata de (III). O item (a) do Fato 2.41 garante a naturalidade da construção, justificando a sua inclusão no enunciado e completando a prova.

Observamos que se $\mathcal{M}=\left(M_{\mid J} ; \quad\left\{\pi_{K J}: J \subseteq K\right.\right.$ e $\left.\left.J \in \mathcal{F}\right\}\right)$ é o sistema dirigido da proposição acima então a $L$-estrutura $\lim \mathcal{M}$ parece ser a noção "fundamental" de produto reduzido (ou ultraproduto, quando $\mathcal{F}$ for ultrafiltro) pois é esta estrutura que sempre satisfaz o teorema de Lós e sua versão para produtos reduzidos (ver 2.23 e comentário subsequente) ao passo que, se permitirmos alguma estrutura vazia $M_{j}$ na definição original de produto reduzido, tomarmos algum filtro tal que $\{j\} \notin \mathcal{F}$ e considerarmos a sentença "eu não sou a estrutura vazia" : $\exists v_{0}\left(v_{0}=v_{0}\right)$, temos que $\prod_{i \in I} M_{i} / \mathcal{F}$ é vazia mas $\left\{i \in I: M_{i}\right.$ é vazia $\} \notin \mathcal{F}$.

\subsection{Morfismos Puros}

Nesta seção apresentamos algumas propriedades fundamentais de L-morfismos puros, importantes em todo trabalho.

Definição 2.42. Seja L uma linguagem de primeira ordem com igualdade. Um L-morfismo, $f: M \longrightarrow N$, é puro se para toda $\varphi\left(v_{1}, \ldots, v_{n}\right) \in \exists^{+}(L)$ e $\bar{a} \in M^{n}$

$$
M \vDash \varphi[\bar{a}] \Leftrightarrow N \vDash \varphi[f \bar{a}] .
$$

Lema 2.43. Sejam L uma linguagem de primeira ordem com igualdade e $f: M \longrightarrow N u m$ L-morfismo.

a) As seguintes condições são equivalentes :

(1) f é um L-morfismo puro;

(2) Para toda $\varphi\left(v_{1}, \ldots, v_{n}\right) \in \exists^{+}(L)$ e todo $\bar{a} \in M^{n}, \quad N \vDash \varphi[f \bar{a}] \Rightarrow M \vDash \varphi[\bar{a}]$;

(3) Para toda $\psi\left(v_{1}, \ldots, v_{n}\right) \in \operatorname{pp}(L)$ e todo $\bar{a} \in M^{n}, \quad N \vDash \psi[f \bar{a}] \Rightarrow M \vDash \psi[\bar{a}]$.

b) Todo morfismo puro é uma L-imersão.

c) Toda L-imersão elementar é pura.

\section{Prova.}

a) A equivalência entre (1) e (2) segue de 2.16.(a), já que $L$-morfismos preservam fórmulas existenciais positivas. A equivalência entre (1) e (3) vem do fato que $p p(L) \subseteq \exists^{+}$e do Lema 2.9 que garante que toda fórmula existencial positiva é equivalente a uma disjunção de conjunções de fórmulas em $\operatorname{pp}(L)$. 
b) Como fórmulas atômicas estão em $\operatorname{pp}(L)$, segue da equivalência em (a) e de 2.15.(3) que todo $L$-morfismo puro é uma $L$-imersão; em particular, é injetor. O item (c) está claro.

Corolário 2.44. Seja $\Sigma$ um conjunto de sentenças da forma $\left.\forall \vec{x}\left(\psi_{0}(\vec{x}) \rightarrow \psi_{1}(\vec{x})\right)\right)$, onde $\psi_{0}(\vec{x})$, $\psi_{1}(\vec{x}) \in \exists^{+}(L)$. Se $N \in \operatorname{Mod}(\Sigma), M$ é uma L-estrutura e existe um L-morfismo puro de $M$ em $N$, então $M \in \operatorname{Mod}(\Sigma)$.

Prova. Segue imediatamente da equivalência no Lema 2.43.(a).

Exemplos do fenômeno indicado no Corolário 2.44 podem ser obtidos a partir do Teorema 2.50 , discutido na próxima seção.

Proposição 2.45. Seja L uma linguagem de primeira ordem com igualdade.

a) Sejam $M \stackrel{f}{\rightarrow} N \stackrel{g}{\rightarrow} P$ L-morfismos. Então :

(1) $f, g$ puros $\Rightarrow g$ of puro;

(2) $g \circ f$ puro $\Rightarrow f$ puro.

Em particular, toda coretração (1.15.(d)) em L-mod é pura.

b) Se $f_{i}: M_{i} \longrightarrow N_{i}, i \in I$, é uma família de L-morfismos puros, o mesmo é verdade para o seu produto, $\prod_{i \in I} f_{i}: \prod_{i \in I} M_{i} \rightarrow \prod_{i \in I} N_{i}$.

c) ${ }^{\natural}$ Sejam $\langle I, \leq\rangle$ um conjunto dirigido $e$

$$
\mathcal{M}=\left\langle M_{i} ;\left\{f_{i j}: i \leq j\right\}\right\rangle \text { e } \mathcal{N}=\left\langle N_{i} ;\left\{g_{i j}: i \leq j\right\}\right\rangle
$$

sistemas dirigidos em L-mod sobre I. Sejam $\underset{\lim }{\mathcal{M}}=\left\langle M ; f_{i}\right\rangle$ e $\lim \mathcal{N}=\left\langle N ; g_{i}\right\rangle$ os colimites correspondentes em L-mod. Sejam $\left\langle\vec{h}_{i}\right\rangle_{i \in I}: \mathcal{M} \longrightarrow \mathcal{N}$ um morfismo de sistemas dirigidos e $\underset{\lim }{\rightarrow} h_{i}=h: M \longrightarrow N$ o L-morfismo induzido nos colimites.

(1) Se cada $h_{i}$ é puro, o mesmo é verdade para $h: M \longrightarrow N$;

(2) Se $f_{i j}$ é puro, para todo $i \leq j$ em $I$, então $f_{i}: M_{i} \longrightarrow M$ é puro.

d) "Para um L-morfismo, $f: M \longrightarrow N$, as seguintes condiçốes são equivalentes :

(1) $f$ é puro;

(D)
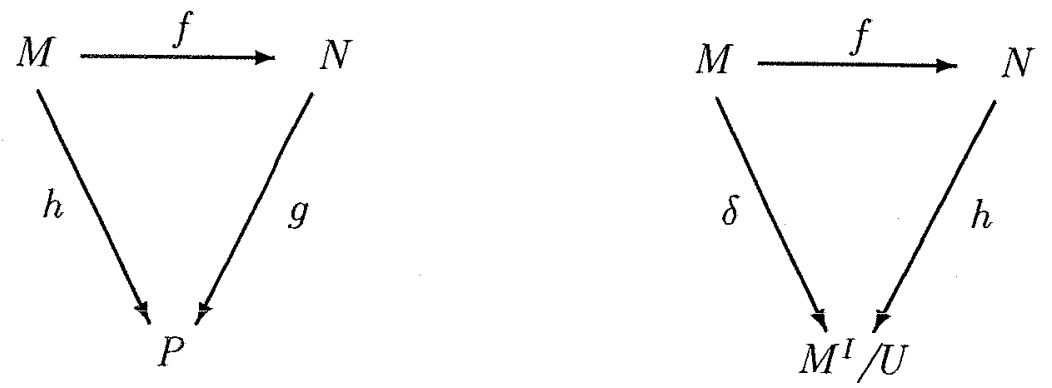
(2) Existe uma L-estrutura $P$, juntamente com L-morfismos $h: M \longrightarrow P e$ $g: N \longrightarrow P$ tal que o diagrama à esquerda em $(D)$ é comutativo e $h$ é um L-morfismo puro;

(3) Existe uma L-estrutura $P$ e L-morfismos $h: M \longrightarrow P$ e $g: N \longrightarrow P$ tais que o diagrama à esquerda em $(D)$ é comutativo e h é uma L-imersão elementar.

(4) Existem um conjunto $I$, um ultrafiltro $U$ em $I$ e um L-morfismo $h: N \longrightarrow M^{I} / U$ tal que o diagrama à direita em $(D)$ é comutativo, onde $\delta$ é o L-morfismo diagonal de $M$ na ultrapotência $M^{I} / U$.

\section{Prova.}

a) Consequência imediata de 2.43.(a). b) Segue facilmente do item (b) em 2.20 que se $\varphi\left(v_{1}, \ldots, v_{n}\right) \in \operatorname{pp}(L)$ e $\bar{x}_{1}, \ldots, \bar{x}_{n} \in \prod_{i \in I} M_{i}$,
então

$$
\prod_{i \in I} M_{i} \vDash \varphi\left[\bar{x}_{1}, \ldots, \bar{x}_{n}\right] \quad \text { sse } \quad \forall i \in I, \quad M_{i} \vDash \varphi\left[x_{1 i}, \ldots, x_{n i}\right] .
$$

Esta observação e 2.43. (a) garantem que o produto de $L$-morfismos puros é puro.

c) (1) Seja $\exists z_{1} \ldots z_{m} \varphi\left(v_{1}, \ldots, v_{n}\right) \in \mathrm{pp}(L)$, onde $\varphi$ é uma conjunção de fórmulas atômicas. Suponha que para $\bar{x} \in M^{n}$ tenhamos $N \models \exists z_{1} \ldots z_{m} \varphi[h(\bar{x})]$. Logo, existem $t_{1}, \ldots ; t_{m} \in N^{m}$ tais que $N=\varphi\left[h(\bar{x}) ; t_{1}, \ldots, t_{m}\right]$. Pela propriedade [colim 2] em 2.37, o fato que $\langle I, \leq\rangle$ é dirigido e que $h=\lim _{\rightarrow}\left\langle h_{i}\right\rangle{ }^{20}$ podemos encontrar $k \in I, \bar{y} \in M_{k}^{n} \mathrm{e}\left\langle w_{1}, \ldots, w_{m}\right\rangle \in N_{k}^{m}$ tais que

$$
\left\{\begin{array}{l}
\left(\text { i) } f_{k}(\bar{y})=\bar{x}\right. \\
\text { (ii) } g_{k}\left(h_{k}(\bar{y})\right)=h(\bar{x}) \\
\text { (iii) } g_{k}\left(w_{p}\right)=t_{p}, 1 \leq p \leq m \\
\text { (iv) } N_{k} \models \varphi\left[h_{k}(\bar{y}) ; w_{1}, \ldots, w_{m}\right]
\end{array}\right.
$$

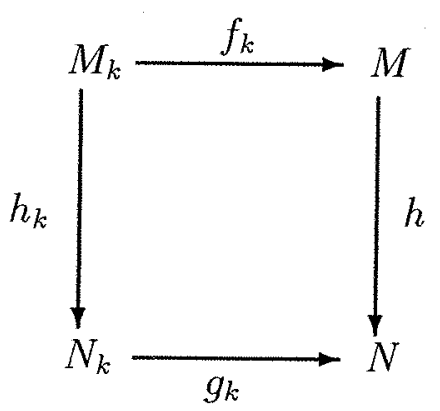

Como $h_{k}$ é puro, $(i v)$ em $\left(^{*}\right)$ implica que $M_{k} \vDash \exists z_{1} \ldots z_{m} \varphi[\bar{y}]$. Uma vez que $\varphi$ é uma conjunção de atômicas, uma nova aplicação de [colim 2] em 2.37 acarreta $M \models \exists z_{1} \ldots z_{m} \varphi\left[f_{k}(\bar{y})\right]$, que por (i) em $\left(^{*}\right)$ é equivalente a $M \models \exists z_{1} \ldots z_{m} \varphi[\bar{x}]$, estabelecendo a puridade de $h$. O item (2) pode ser obtido por método análogo.

d) Mostraremos que (1) $\Rightarrow(3)$. Uma vez isto feito, note que :

$*(2) \Rightarrow(1)$ é consequência do item (2) em (a);

* $(3) \Rightarrow(2)$ vem do fato que imersões elementares são puras;

$*(4) \Rightarrow(3)$ é óbvio, enquanto que $(3) \Rightarrow(4)$ é consequência do Lema de Scott $(2.25)$.

Adicionamos a $L$ um conjunto de novas constantes, duas a duas distintas,

$$
K=\{\underline{a}: a \in M\} \cup\{\underline{b}: b \in N \backslash f(M)\} .
$$

\footnotetext{
${ }^{20}$ Veja o diagrama no enunciado da Proposição 2.38.
} 
Seja $L_{K}$ a linguagem de primeira ordem com igualdade construída com o alfabeto de $L$ e $K$. Indicamos por $L_{M}$ o reduto de $L_{K}$, obtido eliminando-se do alfabeto as constantes referentes aos elementos de $N \backslash f(M)$. Para fazermos de $M$ e $N L_{K}$-estruturas basta indicar a interpretação das constantes em $K$. Seja $a_{0}$ um elemento de $M .{ }^{21}$

* Se $a \in M$, então $\underline{a}^{M}=a$; se $b \in N \backslash f(M)$, então $\underline{b}^{M}=a_{0}$;

* Se $a \in M$, então $\underline{a}^{N}=f(a)$; se $b \in N \backslash f(M)$, definimos $\underline{b}^{N}=b$.

Se $\psi$ é uma sentença em $L_{K}$, escrevemos

$$
\psi\left(\underline{a_{1}}, \ldots, \underline{a_{n}} ; \underline{b_{1}}, \ldots \underline{b_{m}}\right)
$$

para indicar que as novas constantes que aparecem em $\psi$ estão entre as $\underline{a_{j}} \operatorname{com} a_{j} \in M$, e $\underline{b_{i}}$, com $b_{i} \in N \backslash f(M)$. Consideremos

$$
\begin{aligned}
\Sigma & =\left\{\sigma \in \operatorname{Sent}\left(L_{M}\right): M \vDash \sigma\right\} \cup\left\{\varphi \in \operatorname{Sent}\left(L_{K}\right): \varphi \text { é atômica e } N \vDash \varphi\right\} \\
& =\mathcal{T}(M) \cup\left\{\varphi \in \operatorname{Sent}\left(L_{K}\right): \varphi \text { é atômica e } N \vDash \varphi\right\}
\end{aligned}
$$

onde $\mathcal{T}(M)$ é a teoria completa de $M$ (2.18.(c)). Mostraremos que $\Sigma$ é um conjunto consistente. Suponha, por absurdo, que $\Sigma$ é inconsistente. Então, já que $\mathcal{T}(M)$ é fechado por conjunções, existe uma sentença $\sigma \in \mathcal{T}(M)$ e sentenças atômicas de $L_{K}, \varphi_{1}, \ldots, \varphi_{n}$, verdadeiras em $N$, tais que

$$
\varphi_{1} \wedge \ldots \wedge \varphi_{n} \vdash_{\mathcal{C}} \neg \sigma .
$$

Com a convenção notacional em (I) acima, seja $\psi\left(\underline{a_{1}}, \ldots, \underline{a_{p}} ; \underline{b_{1}}, \ldots \underline{b_{q}}\right)$ a conjunção das $\varphi_{k}$, $1 \leq k \leq n$. Logo

$$
\psi\left(\underline{a_{1}}, \ldots, \underline{a_{p}} ; \underline{b_{1}}, \ldots \underline{b_{q}}\right) \vdash_{\mathcal{C}} \neg \sigma .
$$

Como as constantes $\underline{b_{1}}, \ldots \underline{b_{q}}$ não aparecem em $\neg \sigma$ (pois esta é uma $L_{M}$-sentença), podemos substitui-las por variáveis $\overline{z_{1}}, \ldots z_{q}$, que não aparecem nem em $\psi$, nem em $\neg \sigma$, nem em uma prova (pré-fixada) de (II). Por indução no comprimento dessa prova de (II) obtemos

$$
\psi\left(\underline{a_{1}}, \ldots, \underline{a_{p}} ; z_{1}, \ldots z_{q}\right) \vdash_{\mathcal{C}} \neg \sigma .
$$

Segue então da Regra $\exists$ em 2.5.(12) que

$$
\exists z_{1} \ldots z_{q} \psi\left(\underline{a_{1}}, \ldots, \underline{a_{p}}\right) \vdash_{\mathcal{C}} \neg \sigma .
$$

Observe que $\exists z_{1} \ldots z_{q} \psi\left(\underline{a_{1}}, \ldots, \underline{a_{p}}\right) \in \operatorname{Sent}\left(L_{M}\right)$, como é o caso de $\sigma$. Uma vez que $\psi$ é verdadeira em $N$, lembrando qual é a interpretação em $N$ das constantes do tipo $\underline{a}, a \in M$, temos que

$$
N \vDash \psi\left[f\left(a_{1}\right), \ldots, f\left(a_{p}\right) ; b_{1}, \ldots, b_{q}\right]
$$

ou seja $N \vDash \exists z_{1} \ldots z_{m} \psi\left[f\left(a_{1}\right), \ldots, f\left(a_{p}\right)\right]$. Como $f$ é um $L$-morfismo puro, concluímos que

$$
M \vDash \exists z_{1} \ldots z_{m} \psi\left[a_{1}, \ldots, a_{p}\right],
$$

isto é, $\exists z_{1} \ldots z_{m} \psi\left(\underline{a_{1}}, \ldots, \underline{a_{p}}\right)$ está em $\mathcal{T}(M)$. Mas isto é impossível, pois juntamente com (IV) implicaria que $\overline{\mathcal{T}}(M)$ é inconsistente. Assim, $\Sigma$ é consistente e portanto, pelo Teorema da Completude (2.13), existe uma $L_{K^{-}}$estrutura $P$ tal que

\footnotetext{
${ }^{21}$ Se $M=\emptyset$ e $f$ for puro então também $N=\emptyset$ ( senão $N \vDash \exists v_{0}\left(v_{0}=v_{0}\right) \log o M \vDash \exists v_{0}\left(v_{0}=v_{0}\right)$ ), portanto $f$ é isomorfismo (vazio) e a condição (3) é imediatamente satisfeita.
} 


$$
P \vDash \Sigma \text {. }
$$

Como $\mathcal{T}(M) \subseteq \Sigma$, segue de 2.19.(c) (e do comentário sobre a sua prova) que a aplicação

$$
h: M \longrightarrow P \text {, dada por } h(a)=\underline{a}^{P}
$$

é uma $L$-imersão elementar. Resta agora definir o $L$-morfismo $g: N \longrightarrow P$ que faça o diagrama em (2) comutar. Como $f$ é uma imersão (2.43.(b)), se $x \in f(M)$ existe um único $a \in M$ tal que $x=f(a)$. Assim, a prescrição

$$
g(x)= \begin{cases}\underline{a}^{P} & \text { se } x=f(a) \in f(M) \\ \underline{x}^{P} & \text { se } x \in N \backslash f(M),\end{cases}
$$

define uma função $g: N \longrightarrow P$. Para mostrar que $g$ é um $L$-morfismo, seja $\varphi\left(v_{1}, \ldots, v_{n}\right)$ uma fórmula atômica em $L$ e suponha que para $\bar{x} \in N^{n}$ tenhamos $N \models \varphi[\bar{x}]$. Podemos escrever a sequência $\left\langle x_{1}, \ldots, x_{n}\right\rangle$ na forma

$$
\left\langle x_{1}, \ldots, x_{n}\right\rangle=\left\langle f\left(a_{1}\right), \ldots, f\left(a_{p}\right) ; b_{1}, \ldots, b_{q}\right\rangle, \text { com } p+q=n .
$$

Portanto, tendo em conta a interpretação em $N$ das constantes $\underline{a}, a \in M$, obtemos

$$
N \vDash \varphi\left(\underline{a_{1}}, \ldots, \underline{a_{p}} ; \underline{b_{1}}, \ldots \underline{b_{q}}\right)
$$

isto é, $\varphi\left(\underline{a_{1}}, \ldots, \underline{a_{p}} ; \underline{b_{1}}, \ldots \underline{b_{q}}\right) \in \Sigma$. Como $P$ é modelo de $\Sigma$, segue que

$$
P \vDash \varphi\left(\underline{a_{1}}, \ldots, \underline{a_{p}} ; \underline{b_{1}}, \ldots \underline{b_{q}}\right)
$$

ou seja

$$
P \vDash \varphi\left[{\underline{a_{1}}}^{P}, \ldots, \underline{a_{p}}{ }^{P} ;{\underline{b_{1}}}^{P}, \ldots, \underline{b_{q}}{ }^{P}\right] .
$$

Mas então a definição de $g$ em $(V)$ fornece $P \models \varphi[g(\bar{x})]$, mostrando que $g$ é um $L$-morfismo. Finalmente, para verificar que $h=g \circ f$, note que se $a \in M$, então

$$
g(f(a))=\underline{a}^{P}=h(a)
$$

completando a demonstração.

Definição 2.46. Seja $F: \mathbf{L}_{1}-\bmod \longrightarrow \mathbf{L}_{2}-\bmod u m$ funtor, onde $L_{1}$ e $L_{2}$ são linguagens de primeira ordem com igualdade.

a) $F$ preserva ultrapotências se para toda $L_{1}$-estrutura $M$, todo conjunto $I$ e todo ultrafiltro $U$ em I,

$$
F \text { leva o diagrama } M \stackrel{\delta}{\longrightarrow} M^{I} / U \text { no diagrama } F(M) \stackrel{\delta}{\longrightarrow} F(M)^{I} / U
$$

onde $\delta$ é o morfismo diagonal. Ou seja, existe um $L_{2}$-isomorfismo natural $h$ 
$h: F\left(M^{I} / U\right) \longrightarrow F(M)^{I} / U$, que faz comutativo o seguinte diagrama:

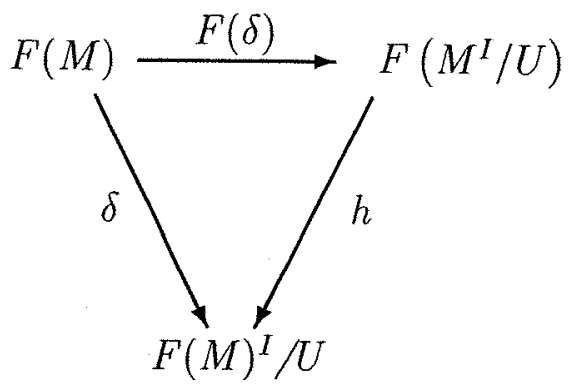

b) $F$ preserva imersões elementares se para toda $L_{1}$-imersão elementar, $M \stackrel{f}{\rightarrow} N, F(f)$ é uma $L_{2}$ imersão elementar.

c) $F$ preserva imersões puras se para todo $L_{1}$-morfismo puro, $M \stackrel{f}{\rightarrow} N, F(f)$ é um $L_{2}$ morfismo puro.

Lema 2.47. Sejam $L$ e $L^{\prime}$ linguagens de primeira ordem com igualdade e $T$ e $T^{\prime}$ conjuntos de sentenças em $L$ e $L^{\prime}$, respectivamente. Se $F: \operatorname{Mod}(T) \longrightarrow \operatorname{Mod}\left(T^{\prime}\right)$ é um funtor, consideremos as seguintes condiçôes :

(1) F preserva ultrapotências;

(2) F preserva imersões elementares;

(3) F preserva imersões puras.

Então: $(1) \Rightarrow(2) \Rightarrow(3)$.

Prova. Por 2.43.(c), temos que (2) $\Rightarrow(3)$. Para (1) $\Rightarrow(2)$, suponha que $f: M \longrightarrow N$ é uma $L$-imersão elementar. Consideremos a linguagem $L_{M}$ gerada adicionando-se novas constantes $\underline{a}, a \in M$, como discutido em 2.17. Então, $M$ é naturalmente uma $L_{M}$-estrutura, com

$$
\underline{a}^{M}=a \text {. }
$$

Seja $\langle M ;\{\underline{a}: a \in M\}\rangle$ essa extensão de $M$ a uma $L_{M^{-}}$estrutura. Fazemos de $N$ uma $L_{M^{-}}$ estrutura, definindo

$$
\underline{a}^{N}=f(a), \quad a \in M,
$$

indicada por $\langle N ;\{f(a): a \in M\}\rangle$. O fato que $f$ é uma $L$-imersão elementar acarreta

$$
\langle M ;\{\underline{a}: a \in M\}\rangle \equiv_{L_{M}}\langle N ;\{f(a): a \in M\}\rangle
$$

isto é, $M$ é $L_{M}$-elementarmente equivalente a $N$. Pelo Teorema de Kiesler-Shelah (2.26) existe um conjunto $I$ e um ultrafiltro $U$ em $I$ tal que

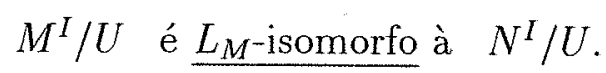

Seja $g: M^{I} / U \longrightarrow N^{I} / U$ um tal $L_{M}$-isomorfismo. Levando em conta a interpretação das constantes $\underline{a}, a \in M$, vemos que o quadrado abaixo à esquerda é comutativo : 

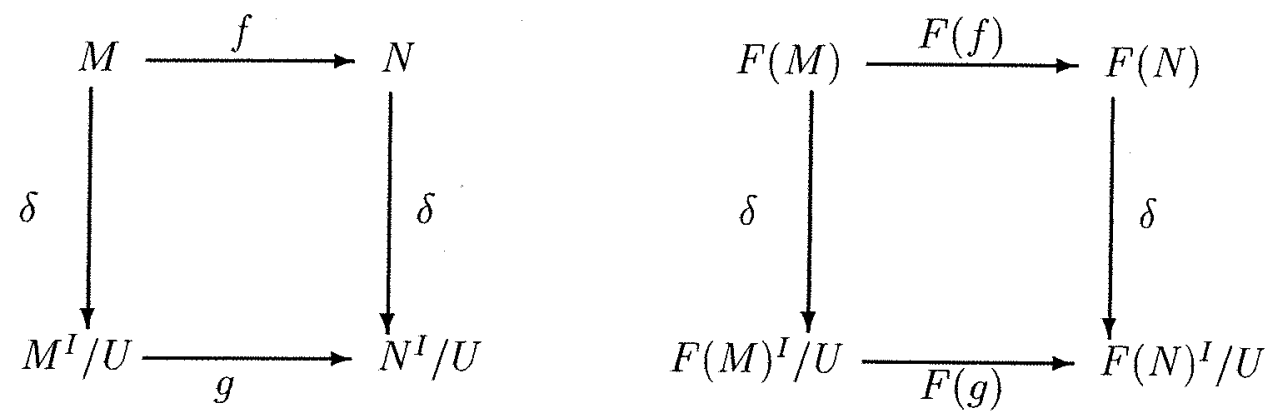

onde $\delta$ indica os morfismos diagonais. Como $F$ preserva ultrapotências e $g$ também é um $L$ isomorfismo, o quadrado acima à direita é comutativo, com $F(g)$ um $L^{\prime}$-isomorfismo. Já que os morfismos diagonais são imersões elementares, é imediato que $F(f)$ é uma $L^{\prime}$-imersão elementar, como desejado.

\subsection{Estruturas Profinitas e Ultraprodutos}

Nesta seção apresentamos uma vasta generalização do Lema 4.4 em [KMS] para a categoria L-mod, onde $L$ é uma linguagem de primeira ordem com igualdade, que permanecerá fixada em tudo que segue.

Definição 2.48. Uma L-estrutura $M$ é profinita se é L-isomorfa ao limite de um sistema cofiltrante de L-estruturas finitas sobre um conjunto parcialmente ordenado e dirigido.

Observação 2.49. Se $P$ é uma $L$-estrutura profinita, existe um conjunto parcialmente ordenado e dirigido, $\langle I, \leq\rangle$, e um sistema cofiltrante de $L$-estruturas finitas sobre $I$,

$$
\mathcal{M}=\left(M_{i} ;\left\{f_{j i}: i \leq j\right\}\right)
$$

tal que $\left\langle P ;\left\{\lambda_{i}: i \in I\right\}\right\rangle=\lim \mathcal{M}$. Pelo Corolário 2.28, podemos considerar $P$ como subestrutura do produto $M=\prod_{i \in I} \overleftarrow{M}_{i}$, isto é, existe um $L$-imersão natural

$$
\iota: P \longrightarrow M
$$

tal que para todo $i \in I$,

$$
\lambda_{i}=\pi_{i} \circ \iota .
$$

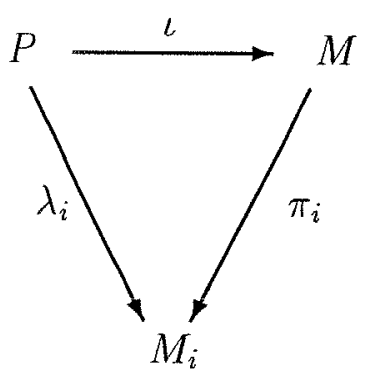


onde $\pi_{i}: M \longrightarrow M_{i}$ é a projeção canônica. Além disso, segue de [lim 1] em $2.28{ }^{22}$ que

$$
\forall \bar{x} \in P \text { e } \forall k \in I, j \in k^{\rightarrow} \Rightarrow f_{j k}\left(x_{j}\right)=x_{k} .
$$

Como observado em 2.22.(5), se $\mathcal{F}$ é um filtro em $I$, para cada $J \in \mathcal{F}$ temos um $L$-morfismo natural

$$
\nu_{J}: M_{\mid J} \longrightarrow M / \mathcal{F} \text {, dado por } x \longmapsto x / \mathcal{F}
$$

onde $M / \mathcal{F}$ indica o produto reduzido $\prod_{i \in I} M_{i} / \mathcal{F}$.

Com estes preliminares enunciamos o

Teorema 2.50. 'L-estruturas profinitas são retratos de ultraprodutos de L-estruturas finitas. De forma mais precisa e com a notação em 2.49 , sejam $\langle I, \leq\rangle$ um conjunto parcialmente ordenado e dirigido $e$

$$
\left.\mathcal{M}=\left(M_{i} ; f_{j i}: i \leq j\right\}\right)
$$

um sistema cofiltrante de estruturas finitas sobre $I$. Se $\lim \mathcal{M}=\left\langle P ;\left\{\lambda_{i}: i \in I\right\}\right\rangle$, entãoo L-morfismo dado pela composiçâo

$$
P \stackrel{\iota}{\longrightarrow} \prod_{i \in I} M_{i} \stackrel{\nu_{I}}{\longrightarrow} \prod_{i \in I} M_{i} / U
$$

é uma coretração (1.15.(d)), onde U é um ultrafiltro dirigido em I (1.4.(c)).

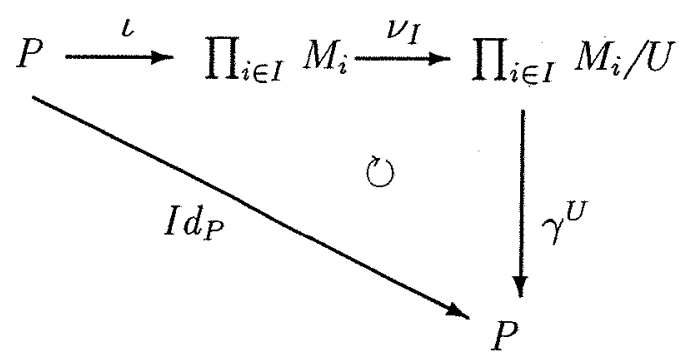

Profinitos e ultraprodutos de finitos

Prova. Pelo Lema 1.6.(a), existe $U \in U l t(I, \leq) \rightarrow$; a prova será feita fixando um tal ultrafiltro. Seja $M=\prod_{i \in I} M_{i}$ a $L$-estrutura produto $\operatorname{dos} M_{i}$.

Pela Proposição 2.40 (e com a mesma notação), sabemos que

$$
M / U=\prod_{i \in I} M_{i} / U \text { é } L \text {-isomorfo à } \underset{\rightarrow}{\lim }\left(M_{\mid J} ;\left\{\pi_{K J}: J \subseteq K \text { e } J \in U\right\}\right) .
$$

Vamos utilizar este fato para construir um $L$-morfismo $\gamma^{U}$ tal que

$$
\gamma^{U} \circ\left(\nu_{I} \circ \iota\right)=I d_{P},
$$

o que completará a prova. Como $U$ permanecerá fixado através da prova, passaremos a indicar $\gamma^{U}$ por $\gamma$. A prova é longa e será feita através de vários Fatos. Além disso, estão em pleno vigor as convenções notacionais de 2.20 e 2.49 .

\footnotetext{
${ }^{22} \mathrm{Com} i=k$.
} 
Para $J \in U, i \in I, \bar{x} \in M_{\mid J}=\prod_{j \in J} M_{j}$ e $y \in M_{i}$, definimos

$$
V_{J, i}(\bar{x}, y)=\left\{j \in J \cap i^{\rightarrow}: f_{j i}\left(x_{j}\right)=y\right\} .
$$

Fato 2.51. Para $J \in U, i \in I, \bar{x} \in M_{\mid J}$ e $y, z \in M_{i}$,

a) $z \neq y \Rightarrow V_{J, i}(\bar{x}, y) \cap V_{J, i}(\bar{x}, z)=\emptyset$.

b) $J \cap i^{\rightarrow}=\coprod_{y \in M_{i}} V_{J, i}(\bar{x}, y) \cdot{ }^{23}$

Prova. O item (a) é consequência imediata do fato que $f_{j i}$ é uma função. Para (b), pela definição de $V_{J, i}(\bar{x}, y)$ é suficiente mostrar que o lado esquerdo de (b) está contido em seu lado direito. Mas note que se $j \in J \cap i^{\rightarrow}$, então $f_{j i}\left(x_{j}\right) \in M_{i}$, como necessário.

Fato 2.52. Para para cada $J \in U$ e $i \in I$, existe um L-morfismo

$$
\gamma_{J, i}: M_{\mid J}=\prod_{k \in J} M_{k} \longrightarrow M_{i}
$$

tal que

a) Se $\bar{x} \in M_{\mid J}$ e $y \in M_{i}$, então $\gamma_{J, i}(\bar{x})=y \quad$ sse $\quad V_{J, i}(\bar{x}, y) \in U$.

b) Se $J \subseteq K$ são elementos de $U$ e $i \in I$, o diagrama abaixo à esquerda é comutativo :
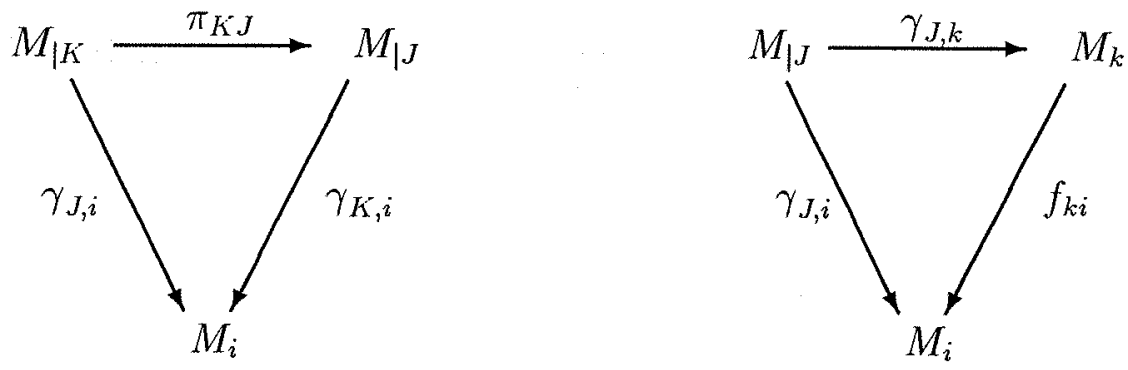

c) Para $J \in U$ e $i \leq k$ em $I$, o diagrama acima à direita é comutativo.

d) Para todo $k \in I, \quad \gamma_{I, k} \circ \iota=\pi_{k} \circ \iota$, onde $\pi_{k}: M \longrightarrow M_{k}$ é a projeção canônica.

Prova. Uma vez que $U \in U l t(I, \leq)^{\rightarrow}$, temos que $J \cap i \rightarrow \in U$ (1.4.(c), 1.6); como este último é um ultrafiltro e $M_{i}$ é finito, 2.51.(b) implica que existe um único $y \in M_{i}$ tal que $V_{J, i}(\bar{x}, y) \in U$. Definimos

$$
\gamma_{J, i}(\bar{x})=\text { o único } y \in M_{i} \text { tal que } V_{J, i}(\bar{x}, y) \in U \text {. }
$$

Está claro que o item (a) está verificado. Devemos agora mostrar que $\gamma_{J, i}$ é um $L$-morfismo. Para facilitar a leitura, se $J \in U$, indicaremos as interpretaçôes dos símbolos de $L$ em $M_{\mid J}$ por um expoente $J$. Assim, se $c$ é uma constante em $L$, utilizaremos $c^{J}$ no lugar de $c^{M_{1 J}}$; analogamente para símbolos funcionais e relacionais. 
* Seja $c \in c t(L)$; lembremos (2.20) que $c^{J}$ é a sequência $\left\langle c^{M_{j}}\right\rangle \in M_{\mid J}$. Assim, como os $f_{j i}$ são $L$-morfismos, obtemos

$V_{J, i}\left(c^{J}, c^{M_{i}}\right)=\left\{j \in J \cap i \rightarrow: f_{j i}\left(c_{j}^{J}\right)=c^{M_{i}}\right\}=\left\{j \in J \cap i \rightarrow: f_{j i}\left(c^{M_{j}}\right)=c^{M_{i}}\right\}=J \cap i \rightarrow$ que está em $U$. Pelo item (a), $\gamma_{J, i}\left(c^{J}\right)=c^{M_{i}}$, como desejado.

* Seja $\omega$ um símbolo relacional $n$-ário em $L$. Se $\bar{x}_{1}, \ldots, \bar{x}_{n} \in\left(M_{\mid J}\right)^{n}$ e $j \in J$, por 2.20 temos que

$$
\omega^{J}\left(\bar{x}_{1}, \ldots, \bar{x}_{n}\right)(j)=\omega^{M_{j}}\left(x_{1 j}, \ldots, x_{n j}\right) .
$$

Sejam

$$
\left\{\begin{array}{rlrl}
y^{p} & =\gamma_{J, i}\left(\bar{x}_{p}\right), & & 1 \leq p \leq n \\
z & =\omega^{M_{i}}\left(y^{1}, \ldots, y^{n}\right) ; & \\
h & =\omega^{J}\left(\bar{x}_{1}, \ldots, \bar{x}_{n}\right) & \left(\in M_{\mid J}\right) .
\end{array}\right.
$$

Mostraremos que

$$
\bigcap_{p=1}^{n} V_{J, i}\left(\bar{x}_{p}, y^{p}\right) \subseteq V_{J, i}(h, z) .
$$

Se $j \in \bigcap_{p=1}^{n} V_{J, i}\left(\bar{x}_{p}, y^{p}\right)$, então a definição de $V_{J, i}$ acarreta

$$
\forall 1 \leq p \leq n, \quad f_{j i}\left(x_{p j}\right)=y^{p} .
$$

Como os $f_{j i}$ são $L$-morfismos, (1) e (3) fornecem

$$
\begin{aligned}
f_{j i}(h(j)) & =f_{j i}\left(\omega^{M_{j}}\left(x_{1 j}, \ldots, x_{n j}\right)\right)=\omega^{M_{i}}\left(f_{j i}\left(x_{1 j}\right), \ldots, f_{j i}\left(x_{n j}\right)\right)=\omega^{M_{i}}\left(y^{1}, \ldots, y^{n}\right) \\
& =z
\end{aligned}
$$

estabelecendo (2). Como a interseção do lado esquerdo de (2) está em $U$, temos $V_{J, i}(h, z) \in U$. Pelo item (a), isto significa que

$$
\gamma_{J, i}\left(\omega^{J}\left(\bar{x}_{1}, \ldots, \bar{x}_{n}\right)\right)=\omega^{M_{i}}\left(\gamma_{J, i}\left(\bar{x}_{1}, \ldots, \bar{x}_{n}\right)\right)
$$

mostrando que $\gamma_{J, i}$ preserva a operação $\omega$;

* Sejam $R$ um símbolo relacional $n$-ário em $L$ e $\bar{x}_{1}, \ldots, \bar{x}_{n} \in\left(M_{\mid J}\right)^{n}$. Por 2.20

$$
M_{\mid J} \vDash R\left[\bar{x}_{1}, \ldots, \bar{x}_{n}\right] \text { sse } \forall j \in J, M_{j} \vDash R\left[x_{1 j}, \ldots, x_{n j}\right] .
$$

Como acima, sejam $y^{p}=\gamma_{J, i}\left(\bar{x}_{p}\right), 1 \leq p \leq n$. Devemos mostrar que

$$
M_{\mid J} \models R\left[\bar{x}_{1}, \ldots, \bar{x}_{n}\right] \quad \Rightarrow \quad M_{i} \models R\left[y^{1}, \ldots, y^{n}\right] .
$$

Como $\bigcap_{p=1}^{n} V_{J, i}\left(\bar{x}_{p}, y^{p}\right) \in U$, essa interseção é não vazia; se $j$ é um elemento dessa interseção, a relação (3) acima está verificada. Logo, segue de (4) e do fato que $f_{j i}$ é um $L$-morfismo que

$$
M_{\mid J} \vDash R\left[\bar{x}_{1}, \ldots, \bar{x}_{n}\right] \quad \Rightarrow \quad M_{j} \vDash R\left[x_{1 j}, \ldots, x_{n j}\right] \quad \Rightarrow \quad M_{i} \vDash R\left[f_{j i}\left(x_{1 j}\right), \ldots, f_{j i}\left(x_{n j}\right)\right],
$$

que por (3) é equivalente à (5), completando a prova de que $\gamma_{J, i}$ é um $L$-morfismo.

b) Sejam $\bar{t} \in M_{\mid K}$ e $\bar{x}=\pi_{K J}(\bar{t})^{24}$. Se $y=\gamma_{J, i}(\bar{x})$, verificaremos que

$$
V_{J, i}(\bar{x}, y) \subseteq V_{K, i}(\bar{t}, y) \text {. }
$$

De fato, se $j \in V_{J, i}(\bar{x}, y)$ (claramente contido em $K \cap i \rightarrow$ ) então

\footnotetext{
${ }^{24}$ Lembramos que $\pi_{K J}$ é a projeção que esquece as coordenadas fora de $K$.
} 


$$
f_{j i}\left(t_{j}\right)=f_{j i}\left(x_{j}\right)=y
$$

como necessário. Uma vez que $V_{J, i}(\bar{x}, y) \in U$, concluímos que $V_{K, i}(\bar{t}, y) \in U$ e o item (a) garante que $\gamma_{K, i}(\bar{t})=y=\gamma_{J, i}\left(\pi_{K J}(\bar{t})\right)$, como necessário.

c) Sejam $\bar{x} \in M_{\mid J}$ e $z=\gamma_{J, k}(\bar{x})$; então,

$$
V_{J, k}(\bar{x}, z) \subseteq V_{J, i}\left(\bar{x}, f_{k i}(z)\right)
$$

De fato, se $j \in V_{J, k}(\bar{x}, z)$ (contido em $J \cap i \rightarrow$ pois $i \leq k$ ), então $f_{j k}\left(x_{j}\right)=z$. Como $\mathcal{M}$ é um sistema cofiltrante, temos

$$
f_{j i}\left(x_{j}\right)=f_{k i}\left(f_{j k}\left(x_{j}\right)\right)=f_{k i}(z)
$$

mostrando que $j \in V_{J, i}\left(\bar{x}, f_{k i}(z)\right)$; já que a relação em (6) garante que esse conjunto está em $U$, o item (a) acarreta $\gamma_{J, i}=f_{k i} \circ \gamma_{J, k}$, como desejado.

d) Para $\bar{x} \in P$ e $k \in I$, note que $\pi_{k}(\iota(\bar{x}))=x_{k}$. Segue da relação (b) em 2.49 (página 74) que

$$
V_{I, k}\left(\iota(\bar{x}), x_{k}\right)=\left\{j \in k^{\rightarrow}: f_{j k}\left(x_{j}\right)=x_{k}\right\}=k^{\rightarrow} .
$$

Já que $U$ é um ultrafiltro dirigido, temos $V_{I, k}\left(\iota(\bar{x}), x_{k}\right) \in U$ e o item (a) garante a conclusão desejada, encerrando a prova do Fato 2.52 .

Pela Proposição 2.40, temos

$$
M / U=\lim _{\longrightarrow}\left\langle M_{\mid J} ;\left\{\pi_{K J}: J \subseteq K, J \in U\right\}\right\rangle
$$

O Fato 2.52.(b) e a propriedade universal dos limites indutivos garantem que, para cada $i \in I$, existe um único $L$-morfismo, $\gamma_{i}: M / U \longrightarrow M_{i}$, tal que para todo $J \in U$ o diagrama abaixo à esquerda é comutativo :
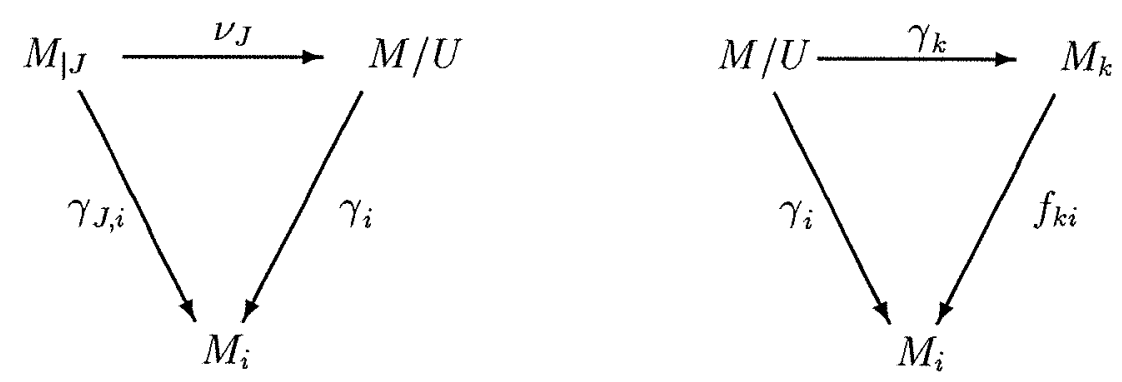

Fato 2.53. Para $i \leq k$ em $I$, o diagrama à direita em $(*)$ acima é comutativo.

Prova. Para $i \leq k$ em $I$ e $J \in U, 2.52$.(c) fornece $\gamma_{J, i}=f_{k i} \circ \gamma_{J, k}$. Assim, a comutatividade do diagrama à esquerda em $\left(^{*}\right)$ - para $k$ e $i-$, implica que para todo $J \in U$ temos

$$
f_{k i} \circ \gamma_{k} \circ \nu_{J}=f_{k i} \circ \gamma_{J, k}=\gamma_{J, i}=\gamma_{i} \circ \nu_{J}
$$

Agora, a unicidade dos $\gamma_{i}$ que fazem o diagrama à esquerda comutativo para todo $J \in U$ garante que $f_{k i} \circ \gamma_{k}=\gamma_{i}$, como desejado. 
O Fato 2.53 mostra que $\left\langle M / U ;\left\{\gamma_{i}: i \in I\right\}\right\rangle$ é um cone sobre o sistema cofiltrante $\mathcal{M}$. Assim a propriedade universal dos limites projetivos garante que existe um único $L$-morfismo

$$
\gamma: M / U \longrightarrow P=\lim \mathcal{M}
$$

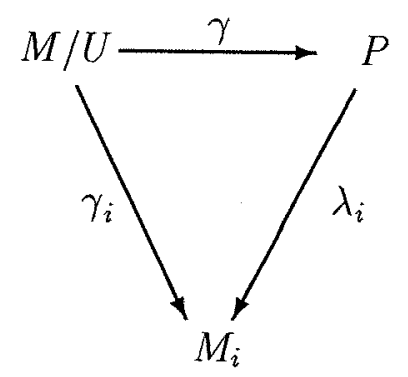

tal que para todo $i \in I$ o diagrama acima é comutativo. Verificaremos que

$$
\gamma \circ \nu_{I} \circ \iota=I d_{P} .
$$

Como $\left\langle P ;\left\{\lambda_{i}: i \in I\right\}\right\rangle=\lim \mathcal{M}$, a propriedade universal dos limites garante que para provar (7) é suficiente mostrar que para todo $k \in I$

$$
\lambda_{k} \circ\left(\gamma \circ \nu_{I} \circ \iota\right)=\lambda_{k}
$$

Já que

$$
\left\{\begin{array}{lll}
\lambda_{k} \circ \gamma=\gamma_{k} ; & \text { Pelo diagrama em }\left({ }^{* *}\right) ; \\
\gamma_{k} \circ \nu_{I}=\gamma_{I, k} ; & \text { Pelo diagrama à esquerda em }\left({ }^{*}\right), \text { pag. } 77 ; \\
\pi_{k} \circ \iota=\lambda_{k} ; & \text { Por }(\sharp) \text { em 2.49, pag. } 74
\end{array}\right.
$$

(8) é equivalente a $\gamma_{I, k} \circ \iota=\pi_{k} \circ \iota$, exatamente o conteúdo de 2.52.(d), completando a prova.

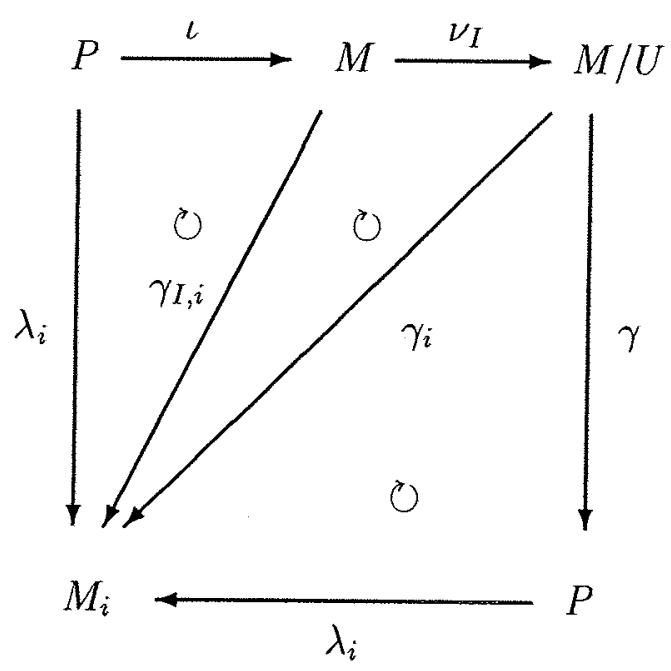

O Corolário 2.44 e o Teorema 2.50 fornecem o seguinte:

Corolário 2.54. ${ }^{\natural}$ Seja $\mathcal{A}=\operatorname{Mod}(T)$ onde $T$ é uma teoria axiomatizável por sentenças $d a$ forma $\left.\forall \vec{x}\left(\psi_{0}(\vec{x}) \rightarrow \psi_{1}(\vec{x})\right)\right)$ onde $\psi_{0}(\vec{x}), \psi_{1}(\vec{x})$ são existenciais positivas. Então a categoria $\mathcal{A}$ 
tem objetos profinitos ${ }^{25}$, isto é, $\mathcal{A}$ é fechada em $\mathbf{L}-\bmod$ por tais limites projetivos.

${ }^{25}$ Notemos que $\mathcal{A}$ de fato possui objetos finitos: $\mathbb{1 1}$, o objeto final de L-mod, está em $\mathcal{A}$, como observado em 2.20 . 


\section{Capítulo 3}

\section{Grupos Especiais}

Este Capítulo descreve algumas das propriedades fundamentais dos Grupos Especiais, que são o tema principal deste trabalho. A referência básica para esta teoria é [DM1].

\subsection{Introdução}

Grupos Especiais constituem uma formulação em linguagem de primeira ordem da Teoria Algébrica das formas quadráticas.

Definição 3.1. (Definição 1.2, [DM1])

(A) Um grupo pré-especial (pSG),

$$
\mathcal{H}=\left(H, \equiv_{H},-1\right),
$$

consiste de

* Um grupo $H$ de expoente $2\left(x^{2}=1\right.$ ), escrito multiplicativamente (logo, 1 é o seu elemento neutro), juntamente com um elemento distinguido -1 .

Escrevemos - a para o produto $-1 \cdot a$;

* Uma relação $\equiv_{H} \subseteq(H \times H) \times(H \times H)$, tal que $\forall a, b, c, d, x \in H:$

[SG0] : $\equiv_{H}$ é uma relação de equivalência;

$[\mathrm{SG} 1]:\langle a, b\rangle \equiv_{H}\langle b, a\rangle$;

$[\mathrm{SG} 2]:\langle a,-a\rangle \equiv_{H}\langle 1,-1\rangle$;

$[\mathrm{SG} 3]:\langle a, b\rangle \equiv_{H}\langle c, d\rangle \Rightarrow a b=c d$;

$[\mathrm{SG} 4]:\langle a, b\rangle \equiv_{H}\langle c, d\rangle \Rightarrow\langle a,-c\rangle \equiv_{H}\langle-b, d\rangle$;

$[\mathrm{SG} 5]:\langle a, b\rangle \equiv_{H}\langle c, d\rangle \Rightarrow\langle x a, x b\rangle \equiv_{H}\langle x c, x d\rangle$.

(B) $\left(H, \equiv_{H},-1\right)$ é um grupo pré-especial reduzido se $\equiv_{H}$ verifica 
[red]

$\forall a \in H,\langle a, a\rangle \equiv_{H}\langle 1,1\rangle \Leftrightarrow a=1{ }^{1}$

(C) Definimos uma extensão $\equiv_{n}$ de $\equiv_{H} a H^{n}, n \in \omega$, como segue :

a) $\equiv_{0}=H^{0} \times H^{0}$; onde a forma vazia, denotada por \langle\rangle , é o único elemento de $H^{0}$;

b) $\equiv_{1}=$ diagonal de $H^{2}$, isto é, $\langle a\rangle \equiv_{1}\langle b\rangle$ sse $a=b$;

c) $\equiv_{2}=\equiv_{H}$;

d) Se $n \geq 2,\left\langle a_{1}, \ldots, a_{n}\right\rangle \equiv_{n}\left\langle b_{1}, \ldots, b_{n}\right\rangle$ sse existem $x, y, z_{3}, \ldots, z_{n}$ em $H$ tais que

(1) $\left\langle a_{1}, x\right\rangle \equiv_{2}\left\langle b_{1}, y\right\rangle$;

(2) $\left\langle a_{2}, \ldots, a_{n}\right\rangle \equiv_{n-1}\left\langle x, z_{3}, \ldots, z_{n}\right\rangle$ e $\left\langle b_{2}, \ldots, b_{n}\right\rangle \equiv_{n-1}\left\langle y, z_{3}, \ldots, z_{n}\right\rangle$.

Quando o contexto permite, deixaremos de mencionar $H$ e $n$ na notação, utilizando

$$
\left\langle a_{1}, \ldots, a_{n}\right\rangle \equiv_{H}\left\langle b_{1}, \ldots, b_{n}\right\rangle \quad \text { ou } \quad\left\langle a_{1}, \ldots, a_{n}\right\rangle \equiv\left\langle b_{1}, \ldots, b_{n}\right\rangle .
$$

Um grup̉o pré-especial é um grupo especial (SG) se verificar

[SG 6] : A extensão de $\equiv_{H} \grave{a} H^{3}$ é transitiva.

Uma relação satisfazendo as condições [SG1] - [SG5] (e [SG6]) denomina-se uma relação pré-especial (respec. especial) em $H$, denominada isometria em $H$.

Definição 3.2. Se $\left\langle H, \equiv_{H},-1\right\rangle$ é um grupo pré-especial, uma forma sobre $H$ é uma n-upla, $\varphi=\left\langle a_{1}, \ldots, a_{n}\right\rangle \in H^{n}$. O natural $n$ denomina-se dimensão de $\varphi$, denotado por $\operatorname{dim}(\varphi)$.

Definição 3.3. Se $H$ é um grupo pré-especial $e \varphi=\left\langle a_{1}, \ldots, a_{n}\right\rangle$ é uma forma sobre $H$ com $n \geq 2$, definimos

$$
D_{H}(\varphi)=\left\{b \in H: \exists b_{2}, \ldots, b_{n} \in H \text { tais que } \varphi \equiv_{H}\left\langle b, b_{2}, \ldots, b_{n}\right\rangle\right\} .
$$

Para $n=0$ temos $D_{H}(\langle\rangle)=\emptyset ;$ no caso $n=1$ temos $D_{H}\left(\left\langle a_{1}\right\rangle\right)=\left\{a_{1}\right\}$.

$D_{H}(\varphi)$ é o denominado o conjunto dos elementos representados por $\varphi$ em $H$. No caso em que $\varphi=\langle a, b\rangle$, escrevemos $D_{H}(a, b)$ no lugar de $D_{H}(\langle a, b\rangle)$.

\section{Observação 3.4.}

a) Por indução em $n \geq 2$, [SG1] - [SG6] implicam que a extensão de $\equiv_{H}$ à $H^{n}$ é uma relação de equivalência para todo $n \geq 0$.

b) É fácil de verificar, utilizando [SG5], que um pSG $H$ é reduzido sse

$$
\forall x, a \in H, \quad x \in D_{H}(a, a) \Rightarrow x=a .
$$

\footnotetext{
${ }^{1}$ Não descartamos aqui estruturas $\mathcal{G}$ tais que $-1=1$. Por [SG2] temos $\mathcal{G} \cong \mathbb{1}$, o grupo especial reduzido com um único elemento.
} 
Como no caso clássico, há operações nos conjuntos de formas :

Definição 3.5. Seja $\langle H, \equiv,-1\rangle$ um grupo pré-especial.

a) Denotamos Form $(H) \doteq \bigcup_{n \in \omega} H^{n}$ é o conjunto das H-formas.

Para $\varphi=\left\langle a_{1}, \ldots, a_{n}\right\rangle \in H^{n}$ e $\psi=\left\langle b_{1}, \ldots, b_{m}\right\rangle \in H^{m}$, definimos soma $e$ produto por :

$$
\left\{\begin{aligned}
\varphi \oplus \psi & \doteq\left\langle a_{1}, \ldots, a_{n}, b_{1}, \ldots, b_{m}\right\rangle \in H^{n+m} \\
\varphi \otimes \psi & \doteq\left\langle a_{1} b_{1}, \ldots, a_{n} b_{1}, a_{1} b_{2}, \ldots, a_{n} b_{2}, \ldots, a_{1} b_{m}, \ldots, a_{n} b_{m}\right\rangle \in H^{n . m}
\end{aligned}\right.
$$

No caso em que $\varphi=\langle a\rangle$ escrevemos a $\psi$ no lugar de $\langle a\rangle \otimes \psi$. Em particular colocamos $-\psi=$ $-1 \psi=\left\langle-b_{1}, \ldots,-b_{m}\right\rangle \in H^{m}$. É fácil ver que para $a, b \in H$

$$
(a b)(\varphi \otimes \psi)=a \varphi \otimes b \psi \text {. }
$$

Se $k$ é um inteiro $\geq 1$ escrevemos $k\langle 1\rangle$ no lugar de $\underbrace{\langle 1\rangle \oplus \ldots \oplus\langle 1\rangle}_{k}$. Abreviamos $k \psi=$ $k\langle 1\rangle \otimes \psi=\underbrace{\psi \oplus \ldots \oplus \psi}_{k} \cdot$ Se $k=0$ convencionamos $k \psi=\langle\rangle$, a H-forma vazia.

b) Se $\varphi=\left\langle a_{1}, \ldots, a_{n}\right\rangle$ é um forma sobre $H$, o produto $d(\varphi)=\prod_{i=1}^{n} a_{i}$ denomina-se o discriminante de $\varphi$.

c) Uma forma de Pfister é uma forma $\varphi$ isométrica a $\bigotimes_{i=1}^{n}\left\langle 1, a_{i}\right\rangle$. O natural n denomina-se grau de $\varphi$. Convencionamos que $\langle 1\rangle$ é a única forma de Pfister de grau 0 . Escrevemos

$$
2^{n}=\bigotimes_{i=1}^{n}\langle 1,1\rangle \text {. }
$$

O contexto evitará qualquer confusão entre o numeral " 2 " e a forma de Pfister $2=\langle 1,1\rangle$.

d) Definimos $\operatorname{Sat}(G)=\bigcup_{n \geq 0} D_{G}\left(2^{n}\right)$.

Definição 3.6. Sejam $\varphi=\left\langle a_{1}, \ldots, a_{n}\right\rangle, \psi=\left\langle b_{1}, \ldots, b_{m}\right\rangle$ formas sobre um grupo especial $G$.

a) $\varphi$ é isotrópica se existe uma forma $\theta$ sobre $G$ tal que $\varphi \equiv\langle 1,-1\rangle \oplus \theta$.

b) $\varphi$ é anisotrópica se nâa for isotrópica.

c) $\varphi$ é hiperbólica se né par e $\varphi \equiv n / 2\langle 1,-1\rangle=\underbrace{\langle 1,-1\rangle \oplus \ldots \oplus\langle 1,-1\rangle}_{n / 2}$.

d) $\varphi$ é subforma de $\psi$ em $G$, indicado por $\varphi \preceq_{G} \psi$, se existe uma forma $\theta$ sobre $G$ tal que

$$
\psi \equiv_{G} \varphi \oplus \theta \text {. }
$$

Está claro que $\varphi \preceq_{G} \psi$ implica $\operatorname{dim} \varphi \leq \operatorname{dim} \psi$.

e) $O$ índice de Witt de $\varphi$, $\operatorname{ind}_{W}(\varphi)$, é definido da seguinte forma

$$
\operatorname{ind}_{W}(\varphi)=\max \left\{k \geq 0: k\langle 1,-1\rangle \preceq_{G} \varphi\right\} .
$$


$S e \operatorname{ind}_{W}(\varphi)=k$, então

$$
\varphi \equiv_{G} k\langle 1,-1\rangle \oplus \varphi_{\text {an }},
$$

onde $\varphi_{a n}$ é uma forma anisotrópica (possivelmente vazia), denominada a parte anisotrópica de $\varphi,{ }^{2}$

Observação 3.7. Note que com a noção de subforma definida em 3.6.(d), se $\varphi$ é uma forma sobre um grupo especial $G$, temos

$$
\left\{\begin{array}{cl}
D_{G}(\varphi) & =\left\{a \in G:\langle a\rangle \preceq_{G} \varphi\right\} ; \\
\varphi \text { é isotrópica } \Leftrightarrow\langle 1,-1\rangle \preceq_{G} \varphi
\end{array}\right.
$$

Lema 3.8. Sejam $\left\langle G, \equiv_{G},-1\right\rangle$ um grupo pré-special, $a, b, c, d \in G$ e $\varphi, \psi$ formas sobre $G$.

a) $\langle a, b\rangle \equiv_{G}\langle c, d\rangle \Leftrightarrow a b=c d \quad e \quad a c \in D_{G}(1, c d)$.

b) $c \in D_{G}(1, a) \Leftrightarrow c\langle 1, a\rangle=\langle c, c a\rangle \equiv_{G}\langle 1, a\rangle$.

c) $\varphi \equiv_{G} \psi \Rightarrow d(\varphi)=d(\psi)$.

Prova. Veja Lema 1.5 em [DM1].

Algumas das propriedades básicas da isometria em grupos especiais aparecem na

Proposição 3.9. Sejam $\left\langle G, \equiv_{G},-1\right\rangle$ um $p S G$ e $\varphi, \psi, \theta$ formas sobre $G$. Então:

a) A soma e o produto de formas preserva isometria, isto é,

$$
\varphi_{1} \equiv_{G} \psi_{1} e \varphi_{2} \equiv_{G} \psi_{2} \quad \Rightarrow \quad \begin{cases}\varphi_{1} \oplus \varphi_{2} \equiv_{G} \psi_{1} \oplus \psi_{2} ; \\ \varphi_{1} \otimes \varphi_{2} \equiv_{G} \psi_{1} \otimes \psi_{2} .\end{cases}
$$

Em particular, isometria é preservada por homotetias, i.e., para todo a $\in G$

$$
\varphi \equiv_{G} \psi \Rightarrow a \varphi \equiv_{G} a \psi \text {. }
$$

Se $G$ é um grupo especial, então

b) (Cancelamento de Witt) $\varphi \oplus \theta \equiv \psi \oplus \theta \Rightarrow \varphi \equiv \psi$.

c) Se a $\in$ e $\varphi_{1}, \ldots, \varphi_{n}$ são formas não vazias sobre $G$,

$a \in D_{G}\left(\bigoplus_{i=1}^{n} \varphi_{i}\right) \quad$ sse $\exists x_{i} \in D_{G}\left(\varphi_{i}\right), 1 \leq i \leq n$, tais que a $\in D_{G}\left(\left\langle x_{1}, \ldots, x_{n}\right\rangle\right)$.

d) Se $\varphi$ e $\psi$ são $G$-formas não vazias então $\varphi \oplus \psi$ é isotrópica sse existe $x \in G$ tal que $x \in$ $D_{G}(\varphi) e-x \in D_{G}(\psi)$. Em particular, para $a \in G, a \in D_{G}(\varphi)$ sse $\langle-a\rangle \oplus \varphi$ é isotrópica.

\footnotetext{
${ }^{2}$ É consequência da propriedade de cancelamento de Witt em 3.9.(b) abaixo, que o índice de Witt de $\varphi$ está bem definido e que $\varphi_{\text {an }}$ é única a menos de isometria.
} 
e) As seguintes condições são equivalentes :

(1) G é um grupo especial reduzido.

(2) Se $\psi$ é uma forma sobre $G, D_{G}(\psi \oplus \psi)=D_{G}(\psi)$.

(3) Se $\psi$ é uma forma sobre $G, \psi \oplus \psi$ isotrópica $\Rightarrow \psi$ isotrópica.

(4) Se $\psi, \theta$ são formas sobre $G, \psi \oplus \psi \equiv \theta \oplus \theta \Rightarrow \psi \equiv \theta$.

(5) $S e \psi$ é uma forma de dimensão par sobre $G, \psi \oplus \psi$ hiperbólica $\Rightarrow \psi$ hiperbólica.

f) Se $\psi$ é uma forma de Pfister sobre $G$, então $D_{G}(\psi)=\left\{x \in G: x \psi \equiv_{G} \psi\right\}$.

g) Se $\varphi, \psi$ são formas de Pfister sobre $G$ então $D_{G}(\varphi) \cup D_{G}(\psi) \subseteq D_{G}(\varphi \otimes \psi)$.

Prova. Veja Proposição 1.6 (para (a) - (e)) e Proposição 2.2.(f) (para (f)) em [DM1]. Para $(\mathrm{g})$, note que se $P$ é uma forma de Pfister, podemos escrevê-la na forma

$$
P=\langle 1\rangle \oplus P^{\prime}
$$

onde $P^{\prime}$ denomina-se a parte pura de $\boldsymbol{P}$. Assim, temos

$$
\varphi \otimes \psi=\varphi \otimes\left(\langle 1\rangle \oplus \psi^{\prime}\right)=\varphi \oplus\left(\varphi \otimes \psi^{\prime}\right)
$$

de onde concluímos imediatamente que $D_{G}(\varphi) \subseteq D_{G}(\varphi \otimes \psi)$. Analogamente, mostra-se que $D_{G}(\psi) \subseteq D_{G}(\varphi \otimes \psi)$.

Caracterizações de grupos especiais são dadas pelo (Teorema 1.23 em [DM1]) :

Teorema 3.10. Para um grupo pré-especial $G$ as seguintes condições são equivalentes :

(1) G é um grupo especial;

(2) Para todo $n \geq 2$ e todas $n$-formas $\varphi$, $\psi$ sobre $G$ e todas as permutações $\sigma$ de $\{1, \ldots, n\}$

$$
\varphi \equiv_{G} \psi \Rightarrow \varphi \equiv_{G} \psi^{\sigma}
$$

onde $\psi^{\sigma}$ é o resultado de aplicar a permutação $\sigma$ às entradas de $\psi ;^{3}$

(3) Para todas as 3 -formas $\varphi$ sobre $G$ e $b_{1}, b_{2}, b_{3} \in G$,

$$
\varphi \equiv_{G}\left\langle b_{1}, b_{2}, b_{3}\right\rangle \quad \Rightarrow \quad \varphi \equiv_{G}\left\langle b_{2}, b_{1}, b_{3}\right\rangle .
$$

Corolário 3.11. Se Gé um grupo especial, a soma e o produto de formas são comutativas, isto é,

$$
\varphi \oplus \psi \equiv_{G} \psi \oplus \varphi \quad e \quad \varphi \otimes \psi \equiv_{G} \psi \otimes \varphi .
$$

Prova. Se $\varphi, \psi$ são formas sobre $G$, temos :

\footnotetext{
${ }^{3}$ Isto é, se $\psi=\left\langle b_{1}, \ldots, b_{n}\right\rangle$, então $\psi^{\sigma}=\left\langle b_{\sigma(1)}, \ldots, b_{\sigma(n)}\right\rangle$.
} 
(a) Como $\varphi \equiv_{G} \varphi$, segue do Teorema 3.10 que se $\varphi^{\sigma}$ é a forma obtida por uma permutação das entradas em $\varphi$, então $\varphi \equiv_{G} \varphi^{\sigma}$;

(b) Uma vez que $\psi \oplus \varphi$ é uma permutação de $\varphi \oplus \psi$, segue de (a) que $\varphi \oplus \psi \equiv_{G} \psi \oplus \varphi$;

(c) Como a multiplicação em $G$ é comutativa, está claro que $\psi \otimes \varphi$ é uma permutação de $\varphi \otimes$ $\psi ;$ assim, segue de (a) que $\varphi \otimes \psi \equiv_{G} \psi \otimes \varphi$.

Proposição 3.12. Seja $G$ um grupo special e $\varphi, \psi$ formas sobre $G$. Se $k \geq 1$ é um inteiro tal que $k \leq \operatorname{dim}(\varphi), \operatorname{dim}(\psi)$ então

$$
k \leq \operatorname{ind}_{W}(\varphi \oplus-\psi) \quad \Rightarrow \quad \begin{aligned}
& \text { Existe uma forma } \theta \text { sobre } G \text { tal que } \operatorname{dim}(\theta)=k, \\
& \theta \preceq_{G} \varphi \text { e } \theta \preceq_{G} \psi .
\end{aligned}
$$

Prova. Vamos utilizar, sem menção expressa, o fato que a soma de formas é comutativa (3.11). Se $k=1$ então $\varphi$ e $\psi$ são não vazias e $\varphi \oplus-\psi$ é isotrópica logo 3.9.(d) garante que existe $x \in$ $G$ tal que

$$
x \in D_{G}(\varphi) \text { e }-x \in D_{G}(-\psi)
$$

que por 3.9.(a) implica $x \in D_{G}(\varphi) \cap D_{G}(\psi)$. Por 3.7, temos $\langle x\rangle \preceq_{G} \varphi, \psi$. Prosseguimos por indução, admitindo o resultado verdadeiro para $k<\operatorname{ind}_{W}(\varphi \oplus-\psi)$; então existe uma forma $\theta$ de dimensão $k$ sobre $G$ tal que

$$
\varphi \equiv_{G} \theta \oplus \varphi_{1} \text { e } \psi \equiv_{G} \theta \oplus \psi_{1} .
$$

Assim, lembrando 3.9.(a) e que $\langle a,-a\rangle \equiv_{G}\langle 1,-1\rangle$, obtemos

$$
\begin{gathered}
\varphi \oplus-\psi \equiv_{G}(k+1)\langle 1,-1\rangle \oplus \chi \equiv_{G}\left(\theta \oplus \varphi_{1}\right) \oplus\left(-\theta \oplus-\psi_{1}\right) \equiv_{G} \quad(\theta \oplus-\theta) \oplus\left(\varphi_{1} \oplus-\psi_{1}\right) \\
\equiv_{G} \quad k\langle 1,-1\rangle \oplus\left(\varphi_{1} \oplus-\psi_{1}\right) .
\end{gathered}
$$

Pelo cancelamento de Witt (3.9.(b)), a relação acima implica

$$
\langle 1,-1\rangle \oplus \chi \equiv_{G} \varphi_{1} \oplus-\psi_{1}
$$

ou seja $\varphi_{1} \oplus-\psi_{1}$ é isotrópica. O mesmo argumento utilizado para o caso $k=1$ mostra que existe $y \in D_{G}\left(\varphi_{1}\right) \cap D_{G}\left(\psi_{1}\right)$. Assim, $\langle y\rangle \oplus \theta$ é uma forma de dimensão $k+1$ que é subforma de $\varphi$ e $\psi$, encerrando a prova.

3.13. O Grupo Especial $\mathbb{Z}_{2}:$ Seja $\mathbb{Z}_{2}=\{1,-1\} \subseteq \mathbb{Z}$, com a sua estrutura multiplicativa usual. Se $n \geq 1$ é um natural e $s \in \mathbb{Z}_{2}^{n}$, seja

$$
p_{s}=\# \text { de } 1 \text { 's em } s \text { e } n_{s}=\# \text { de }-1 \text { 's em } s
$$

Se $s, t \in \mathbb{Z}_{2}^{n}$ definimos $s \equiv_{\mathbb{Z}_{2}} t \quad$ sse $\quad p_{s}=p_{t}$. Com esta 2-relação, $\mathbb{Z}_{2}$ é uma grupo especial reduzido e as $n$-relações definidas coincidem com as $n$-isometrias (3.1.(C)).

Um modo equivalente de definir a relação $\equiv_{\mathbb{Z}_{2}}$ é a seguinte :

$$
s \equiv_{\mathbb{Z}_{2}} t \quad \text { sse } \sum_{i=1}^{n} s(i)=\sum_{i=1}^{n} t(i)
$$


onde $s(i)$ é a $i$-ésima entrada de $s$ e a soma acima é realizada no anel $\mathbb{Z}$.

3.14. A Linguagem dos Grupos Especiais : Indicamos por $L_{S G}$ a linguagem de primeira ordem com igualdade originária da definição de grupo pré-especial (3.1), isto é, cujo alfabeto não lógico é dado por

* Uma relação quaternária, 三, que representa a isometria de formas binárias;

* Uma operação binária, ·, que representa o produto no grupo;

* Duas constantes, $1,-1$.

As vezes é conveniente termos na linguagem um predicado unário para expressar a representação por formas do tipo $\langle 1, a\rangle$, indicado por $D(1, \cdot)$. Note que pelo Lema 3.8.(a) esta linguagem é interdefinível, módulo os axioms de grupo pré-especial, com a estabelecida acima. Assim, quando conveniente, suporemos que um tal predicado está em $L_{S G}$.

Note que se $\varphi, \psi$ são formas de dimensão $n \geq 1$ sobre um grupo pré-special $G$ a afịmação $\varphi \equiv_{G} \psi$ pode ser descrita por uma sentença existencial positiva, $\sigma$, na linguagem $L_{S G}$ (possivelmente muito longa), tendo como parâmetros os coeficientes de $\varphi$ e $\psi$. Para obter $\sigma$ basta seguir os passos indutivos da definição da extensão da relação quaternária básica descrita no item $(C)$ da Definição 3.1 (página 82). Por exemplo, se $\varphi=\left\langle a_{1}, a_{2}, a_{3}\right\rangle$ e $\psi=\left\langle b_{1}, b_{2}, b_{3}\right\rangle$ então:

$$
\sigma=\exists x y z\left(\left\langle a_{1}, x\right\rangle \equiv\left\langle b_{1}, y\right\rangle \wedge\left\langle a_{1}, a_{2}\right\rangle \equiv\langle x, z\rangle \wedge\left\langle b_{1}, b_{2}\right\rangle \equiv\langle y, z\rangle\right) .
$$

Analogamente, podemos expressar as seguintes noções por sentenças existenciais positivas em $L_{S G}$, tendo como parâmetros as entradas das formas envolvidas :

(i) " $\varphi$ é uma forma de Pfister" (3.5.(c));

(ii) A relação " $\varphi$ é subforma de $\psi$ ", isto é, $\varphi \preceq \psi$;

(iii) " $\varphi$ é uma forma é isotrópica";

(iv) "ind $W(\varphi) \geq k$ ", onde $k \geq 0$ é um inteiro.

\subsection{Morfismos}

Definição 3.15. Um morfismo de grupos pré-especiais (pSG-morfismo),

$$
\left\langle G, \equiv_{G},-1\right\rangle \stackrel{f}{\longrightarrow}\left\langle H, \equiv_{H},-1\right\rangle
$$

é um $L_{S G}$-morfismo ${ }^{4}$, ou seja, é um homomorfismo de grupos tal que $f(-1)=-1$ e para todo $a, b, c, d \in G$

$$
\langle a, b\rangle \equiv_{G}\langle c, d\rangle \Rightarrow\langle f(a), f(b)\rangle \equiv_{H}\langle f(c), f(d)\rangle .
$$

\footnotetext{
${ }^{4}$ Ver 2.14.(a) .
} 
Um morfismo de grupos especiais (SG-morfismo) é um morfismo dos grupos pré-especiais subjacentes.

Se $\varphi=\left\langle a_{1}, \ldots, a_{n}\right\rangle$ é uma $n$-forma sobre $G$, indicamos por $f \star \varphi$ a forma $\left\langle f\left(a_{1}\right), \ldots, f\left(a_{n}\right)\right\rangle$ sobre $H$, denominada forma imagem de $\varphi$ por $f$.

Está claro que grupos pré-especiais e seus morfismos, grupos especiais e seus morfismos, bem como grupos especiais reduzidos e seus morfismos constituem categorias, indicadas por PSG, SG e RSG, respectivamente.

Observação 3.16. Se $f: G \longrightarrow H$ é um pSG-morfismo e $\varphi, \psi$ são formas sobre $G$ então

$$
f \star(\varphi \oplus \psi)=(f \star \varphi) \oplus(f \star \psi) \quad \text { e } f \star(\varphi \otimes \psi)=(f \star \varphi) \otimes(f \star \psi) .
$$

Em particular, para todo $a \in G, f \star a \varphi=f(a)(f \star \varphi)$; assim, $f \star-\varphi=-(f \star \varphi)$.

Lema 3.17. Sejam $G ; H$ grupos pré-especiais e $f, g: G \longrightarrow H$ tais que $f$ é uma função e $g$ é um $p S G$-morfismo.

a) $f$ é um $p S G$-morfismo sse for um homomorfismo de grupos tal que $f(-1)=-1$ e verificar

$$
\forall a, b \in G, a \in D_{G}(1, b) \quad \Rightarrow \quad f(a) \in D_{H}(1, f(b)) .
$$

b) Uma função $\sigma: G \longrightarrow \mathbb{Z}_{2}$ é um $p S G$-morfismo sse é um homomorfismo de grupos que leva -1 em-1 e verifica

[ker]

$$
\forall a \in G, a \in \operatorname{ker} \sigma \quad \Rightarrow \quad D_{G}(1, a) \subseteq \operatorname{ker} \sigma .
$$

c) $\operatorname{Se} \varphi, \psi$ são formas sobre $G$ de mesma dimensão, então

$$
\varphi \equiv_{G} \psi \Rightarrow g \star \varphi \equiv_{H} g \star \psi \text {. }
$$

Prova. Veja Lema 1.12 em [DM1].

\subsection{SG-carácteres. Espaço de Ordens. Assinatura.}

a) Se $H$ é um grupo especial, os SG-morfismos $H \stackrel{\sigma}{\longrightarrow} \mathbb{Z}_{2}$ são denominados $\mathbf{S G - c a r a ́ c t e r e s ~ d e ~}$ H. Seja

$$
X_{H}=\{\sigma: \sigma \text { é um SG-carácter de } H\},
$$

o espaço de ordens de $H$. Podemos considerar $X_{H}$ como subconjunto de $2^{H}$. Se dermos a $2^{H}$ a topologia produto, então $X_{H}$ é um fechado no espaço Booleano $2^{H}$. Assim, com a topologia induzida pela topologia produto em $2^{H}, X_{H}$ é também um espaço Booleano. Esta topologia em $X_{H}$ tem como base a interseção finita de subconjuntos da forma

$$
[a=1]=\left\{\sigma \in X_{H}: \sigma(a)=1\right\}, \quad(a \in H) .
$$


Assim, todo aberto em $X_{H}$ é reunião de interseções do tipo $\left[a_{1}=1\right] \cap \ldots \cap\left[a_{n}=1\right]$, com $\left\{a_{1}, \ldots, a_{n}\right\} \subseteq H$.

Além disso, para cada SG-morfismo $f: G \longrightarrow G^{\prime}$, temos função contínua :

$$
X_{f}: X_{G^{\prime}} \longrightarrow X_{G} \text {, dada por } \sigma^{\prime} \mapsto \sigma^{\prime} \circ f \text {. }
$$

De fato, temos funtor contravariante $X_{(?)}: \mathbf{S G} \longrightarrow \mathbf{B S}$, onde $\mathbf{B S}$ designa a categoria dos espaços booleanos e funções contínuas.

b) Se $\varphi=\left\langle a_{1}, \ldots, a_{n}\right\rangle$ é uma forma sobre $H$ e $\sigma \in X_{H}$, a assinatura de $\varphi$ em $\sigma$ é o inteiro ${ }^{5}$

$$
\sigma(\varphi)=\sum_{i=1}^{n} \sigma\left(a_{i}\right)
$$

É fácil ver que a assinatura comuta com a soma e o produto de formas, isto é, se $\varphi$, $\psi$ são formas sobre $H$ e $\sigma \in X_{H}$

$$
\sigma(\varphi \oplus \psi)=\sigma(\varphi)+\sigma(\psi) \quad \text { e } \quad \sigma(\varphi \otimes \psi)=\sigma(\varphi) \sigma(\psi)
$$

Se $H$ é um grupo especial reduzido, vale a seguinte generalização do famoso Princípio LocalGlobal de Pfister :

Teorema 3.19. Sejam $\varphi, \psi$ n-formas sobre um grupo especial reduzido $H$. Então

$$
\varphi \equiv_{H} \psi \text { sse } \forall \sigma \in X_{H}\left(\sigma \star \varphi \equiv_{\mathbb{Z}_{2}} \sigma \star \psi\right),
$$

ou, de forma equivalente, $\forall \sigma \in X_{H}, \quad \sum_{i=1}^{n} \sigma\left(a_{i}\right)=\sum_{i=1}^{n} \sigma\left(b_{i}\right)$, onde esta soma é calculada em $\mathbb{Z}, \operatorname{com} \varphi=\left\langle a_{1}, \ldots, a_{n}\right\rangle$ e $\psi=\left\langle b_{1}, \ldots, b_{n}\right\rangle$.

Prova. Veja Proposição 3.7 em [DM1].

3.20. Alguns tipos de SG-morfismos: No Capítulo 5 de [DM1] há um estudo detalhado de diversos tipos de morfismos entre grupos especiais ${ }^{6}$. A lista abaixo destaca os que serão importantes no que segue. Seja $f: G \longrightarrow H$ um SG-morfismo. Dizemos que $f$ é

a) Uma imersão completa se para quaisquer formas $\varphi, \psi$ de mesma dimensão sobre $G$

$$
\varphi \equiv_{G} \psi \Leftrightarrow f \star \varphi \equiv_{H} f \star \psi
$$

b) Reflete isotropia se para toda forma $\varphi$ sobre $G$

$$
f \star \varphi \text { isotrópica em } H \Rightarrow \varphi \text { isotrópica em } G \text {. }
$$

c) $)^{\natural}$ Reflete subformas se para quaisquer formas $\varphi, \psi$ sobre $G$

$$
f \star \varphi \preceq_{H} f \star \psi \Rightarrow \varphi \preceq_{G} \psi
$$

d) $)^{\natural}$ Reflete índice de Witt se para toda forma $\varphi$ sobre $G, \operatorname{ind}_{W}(\varphi)=\operatorname{ind}_{W}(f \star \varphi)$.

\footnotetext{
${ }^{5}$ As vezes a assinatura de $\varphi$ em $\sigma$ é indicada por $\operatorname{sgn} \sigma(\varphi)$.

${ }^{6}$ Que fazem sentido quando consideramos $L_{S G}$-estruturas em geral.
} 
e) Puro se para toda fórmula existencial positiva da linguagem $L_{S G}$ dos grupos especiais (3.14), $\varphi\left(v_{1}, \ldots, v_{n}\right)$ e todo $\bar{g} \in G^{n}, \quad H \vDash \varphi[f(\bar{g})] \quad \Rightarrow \quad G \models \varphi[\bar{g}]$.

\section{Proposição 3.21.}

a) Toda imersão completa é injetora.

b) Se $f: G \longrightarrow H$ é um SG-morfismo, então
$f$ puro $\stackrel{(1)}{\Rightarrow} f$ reflete subformas $\stackrel{(2)}{\Rightarrow} f$ reflete isotropia $\stackrel{(3)}{\Rightarrow} f$ é imersão completa.

c) Sejam $f: G \longrightarrow H$ um SG-morfismo e $\varphi$ uma forma sobre $G$.

(1) $\operatorname{ind}_{W}(\varphi) \leq \operatorname{ind}_{W}(f \star \varphi)$

(2) $f$ reflete subformas $\Leftrightarrow f$ reflete indice de Witt.

\section{Prova.}

a) Seja $f: G \longrightarrow H$ uma imersão completa e suponha que para $a, b \in G$ tenhamos $f(a)=f(b)$; então $f(a b)=1$ e portanto

$$
\langle f(1), f(a b)\rangle \equiv_{H}\langle f(1), f(1)\rangle \quad \Rightarrow \quad\langle 1, a b\rangle \equiv_{G}\langle 1,1\rangle
$$

e o axioma do discriminante ([SG 3], 3.1) acarreta $a b=1$, isto é, $a=b$.

b) A implicação (1) vem do fato, observado em 3.14.(ii), que a relação de ser subforma pode ser expressa por uma fórmula existencial positiva de $L_{S G}$. A implicação (2) está clara; para a prova de (3) veja a Proposição 5.32 em [DM1].

c) $\mathrm{O}$ item (1) é consequência imediata de 3.17.(c) e do fato que para todo natural $k \geq 0$, $f \star k\langle 1,-1\rangle=k\langle 1,-1\rangle$. Em relação a (2) temos :

$\Rightarrow$ : Por (1) é suficiente mostrar que se $\varphi$ é uma forma sobre $G$, então ind ${ }_{W}(f \star \varphi) \leq \operatorname{ind}_{W}(\varphi)$. Suponha, por absurdo, que $\operatorname{ind}_{W}(f \star \varphi)>\operatorname{ind}_{W}(\varphi)=k$. Então,

$$
(k+1)\langle 1,-1\rangle=f \star(k+1)\langle 1,-1\rangle \preceq_{H} f \star \varphi
$$

e portanto, já que $f$ reflete subformas, teríamos $(k+1)\langle 1,-1\rangle \preceq_{G} \varphi$, contra a hipótese que $\operatorname{ind}_{W}(\varphi)=k$.

$\Leftarrow$ : Utilizaremos, sem menção explícita, os resultados em 3.11 e 3.16. Sejam $\theta, \varphi$ formas sobre $G$ tais que $f \star \theta \preceq_{H} f \star \varphi, \operatorname{com} \operatorname{dim} \theta=k \geq 1$. Então existe uma forma $\chi$ sobre $H$ tal que

$$
f \star \varphi \equiv_{H}(f \star \theta) \oplus \chi \text {. }
$$

Considere a forma $\varphi_{1}=\varphi \oplus(-\theta)$; então, $\left({ }^{*}\right)$ acarreta

$$
f \star \varphi_{1}=(f \star \varphi) \oplus-(f \star \theta) \equiv_{H} \quad(f \star \theta) \oplus-(f \star \theta) \oplus \chi \equiv_{H} \quad k\langle 1,-1\rangle \oplus \chi,
$$

isto é, $\operatorname{ind}_{W}\left(f \star \varphi_{1}\right) \geq k$. Como $f$ reflete índice de Witt, concluímos que $\operatorname{ind}_{W}\left(\varphi_{1}\right) \geq k$. Pela Proposição 3.12 , existe uma forma $\psi$ de dimensão $k$ sobre $G$ tal que

\footnotetext{
${ }^{7}$ Ou seja $f$ é $L_{S G}$-puro como na Definição 2.42 .
} 


$$
\psi \preceq_{G} \varphi \text { e } \psi \preceq_{G} \theta .
$$

Como $\operatorname{dim} \psi=\operatorname{dim} \theta=k$, temos $\psi \equiv_{G} \theta$ e 3.9.(a) implica que $\theta \preceq_{G} \varphi$, como desejado.

Observação 3.22. Seja $G$ um grupo especial e $H$ um subgrupo de $G$ tal que $-1 \in H$.

a) Com a estrutura induzida por $G,\langle H, \equiv,-1\rangle$ é um grupo pré-especial, denominado subgrupo pré-especial de $G$. Se $H$ for um grupo especial com a estrutura induzida, dizemos que $H$ é um subgrupo especial de $G$.

b) Seja $\iota: H \longrightarrow G$ o pSG-morfismo canônico. Dizemos que $H$ é um subgrupo completo de $G$ se $\iota$ é uma imersão completa, isto é, se $\varphi, \psi$ são formas de mesma dimensão sobre $H$

$$
\varphi \equiv_{H} \psi \Leftrightarrow \varphi \equiv_{G} \psi
$$

Analogamente definimos subgrupo puro e refletor de subformas. É fácil verificar que todo subgrupo pré-especial completo de um grupo especial é um grupo especial. Em particular, todo subgrupo pré-especial puro de um grupo especial é especial (3.21.(b)).

c) Convém registrar que existem subgrupos especiais de um grupo especial que não são completos (Exemplo $5.10 \mathrm{em}[\mathbf{D M 1}]$ ).

O mesmo método utilizado na prova da Proposição 3.12 fornece

Proposição 3.23. Seja $f: G \longrightarrow H$ um SG-morfismo. Considere as seguintes condiçốes :

(1) $f$ reflete isotropia;

(2) $f$ é injetor e para toda forma $\psi$ sobre $G, f\left(D_{G}(\psi)\right)=D_{H}(f \star \psi) \cap \operatorname{Im} f$;

(3) $f$ é injetor e para toda $G$-forma $\psi$ e $a \in G,\langle a\rangle \preceq_{G} \psi \Leftrightarrow\langle f(a)\rangle \preceq_{H} f \star \psi$.

Então: $(1) \Rightarrow(2) \Leftrightarrow(3)$.

O resultado que segue, importante em aplicações futuras, generaliza o Lema 1.3.6 em [Lim].

Teorema 3.24. Sejam $G$ um grupo especial e $H$ um subgrupo pré-especial de G. Suponha que para $H$-formas $\psi_{1}, \psi_{2}$ temos :

$$
D_{G}\left(\psi_{1}\right) \cap D_{G}\left(\psi_{2}\right) \neq \emptyset \Rightarrow H \cap D_{G}\left(\psi_{1}\right) \cap D_{G}\left(\psi_{2}\right) \neq \emptyset
$$

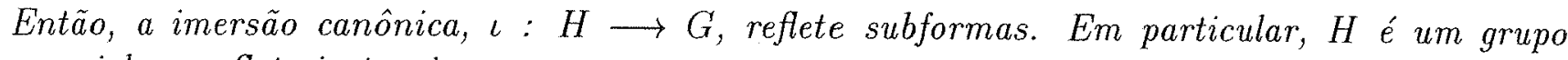
especial e $\iota$ reflete isotropia.

Prova. No Lema 1.3.6 de [Lim] é estabelecido que $H$ é um subgrupo completo de $G$. Iremos precisar do seguinte 
Fato 3.25. Sejam $\alpha=\left\langle\alpha_{1}, \ldots, \alpha_{n}\right\rangle$ uma $H$-forma e $\beta=\left\langle\beta_{1}, \ldots, \beta_{n}\right\rangle$ uma $G$-forma, $n \geq 2$.

a) Suponha que para $a, b \in H$, exista uma $G$-forma $\gamma$ tal que

$$
\alpha \equiv_{G}\langle a\rangle \oplus \gamma \text { e } \beta \equiv_{G}\langle b\rangle \oplus \gamma .
$$

Então, existe uma $H$-forma $\chi$ tal que $\alpha \equiv_{G}\langle a\rangle \oplus \chi \quad$ e $\beta \equiv_{G}\langle b\rangle \oplus \chi$.

b) Se $\alpha \equiv_{G} \beta$ e $\beta_{1} \in H$, então existem $u, v \in H$ e uma $H$-forma $\chi$ tais que

$$
\left\langle\alpha_{1}, u\right\rangle \equiv_{G}\left\langle\beta_{1}, v\right\rangle,\left\langle\alpha_{2}, \ldots, \alpha_{n}\right\rangle \equiv_{G}\langle u\rangle \oplus \chi \quad \text { e }\left\langle\beta_{2}, \ldots, \beta_{n}\right\rangle \equiv_{G}\langle v\rangle \oplus \chi
$$

Prova.

a) Seja $n=\operatorname{dim} \alpha=\operatorname{dim} \beta \geq 2$. Se $n=2$, então $\gamma=\langle x\rangle, x \in G$. Como $\alpha$ é uma $H$-forma, o axioma do discriminante ([SG3], 3.1) imediatamente implica que $x \in H$. Assim, podemos tomar $\chi=\langle x\rangle$. Prosseguimos por indução; suponha o resultado verdadeiro para $n \geq 2$ e seja $\gamma$ uma $G$-forma de dimensão $n$ tal que

$$
\alpha \equiv_{G}\langle a\rangle \oplus \gamma \text { e } \beta \equiv_{G}\langle b\rangle \oplus \gamma .
$$

Se escrevemos $\alpha=\left\langle\alpha_{1}, \ldots, \alpha_{n}, \alpha_{n+1}\right\rangle$, a primeira isometria em (1) fornece ${ }^{8} x, y \in G$ e uma $G$-forma $\lambda$ de dimensão $n-1$ tais que

$$
\left\langle\alpha_{1}, x\right\rangle \equiv_{G}\langle a, y\rangle,\left\langle\alpha_{2}, \ldots, \alpha_{n+1}\right\rangle \equiv_{G}\langle x\rangle \oplus \lambda \text { e } \gamma \equiv_{G}\langle y\rangle \oplus \lambda .
$$

As duas primeiras isometrias em (2) e o axioma [SG4] em 3.1 acarretam

$$
x \in D_{G}\left(\left\langle a,-\alpha_{1}\right\rangle\right) \cap D_{G}\left(\left\langle\alpha_{2}, \ldots, \alpha_{n+1}\right\rangle\right) .
$$

Logo, a condição (RS) implica que existe $u \in H \cap D_{G}\left(\left\langle a,-\alpha_{1}\right\rangle\right) \cap D_{G}\left(\left\langle\alpha_{2}, \ldots, \alpha_{n+1}\right\rangle\right)$. Assim, existem $v \in G$ e uma $G$-forma $\mu$ tais que

$$
\left\langle\alpha_{1}, u\right\rangle \equiv_{G}\langle a, v\rangle \text { e }\left\langle\alpha_{2}, \ldots, \alpha_{n+1}\right\rangle \equiv_{G}\langle u\rangle \oplus \mu .
$$

Note que a primeira isometria em (2) e [SG3] acarretam $v=a u \alpha_{1} \in H$. Por outro lado, a segunda isometria em (2) fornece, lembrando 3.9.(a),

$\langle a\rangle \oplus \gamma \equiv_{G} \alpha=\left\langle\alpha_{1}\right\rangle \oplus\left\langle\alpha_{2}, \ldots, \alpha_{n+1}\right\rangle \equiv_{G}\left\langle\alpha_{1}\right\rangle \oplus\langle u\rangle \oplus \mu=\left\langle\alpha_{1}, u\right\rangle \oplus \mu$

$$
\equiv_{G}\langle a, v\rangle \oplus \mu \equiv_{G}\langle a\rangle \oplus\langle v\rangle \oplus \mu \text {. }
$$

A lei de cancelamento de Witt (3.9.(b)) acarreta $\gamma \equiv_{G}\langle v\rangle \oplus \mu$. Como temos

$$
\left\langle\alpha_{2}, \ldots, \alpha_{n+1}\right\rangle \equiv_{G}\langle u\rangle \oplus \mu \text { e } \gamma \equiv_{G}\langle v\rangle \oplus \mu
$$

com $u, v \in H$ e $\left\langle\alpha_{2}, \ldots, \alpha_{n+1}\right\rangle$ uma $H$-forma de dimensão $n$, a hipótese indução implica que existe uma $H$-forma $\chi$ tal que

$$
\left\langle\alpha_{2}, \ldots, \alpha_{n+1}\right\rangle \equiv_{G}\langle u\rangle \oplus \chi \text { e } \gamma \equiv_{G}\langle v\rangle \oplus \chi
$$

Assim, lembrando a primeira isometria em (2), obtemos

\footnotetext{
${ }^{8}$ Pelo Item (C) em 3.1.
} 


$$
\left\{\begin{array}{c}
\alpha=\left\langle\alpha_{1}\right\rangle \oplus\left\langle\alpha_{2}, \ldots, \alpha_{n+1}\right\rangle \equiv_{G}\left\langle\alpha_{1}, u\right\rangle \oplus \chi \equiv_{G}\langle a, v\rangle \oplus \chi=\langle a\rangle \oplus(\langle v\rangle \oplus \chi) \\
\mathrm{e} \\
\beta \equiv_{G}\langle b\rangle \oplus \gamma \equiv_{G}\langle b\rangle \oplus(\langle v\rangle \oplus \chi),
\end{array}\right.
$$

completando o passo de indução e a prova de (a).

b) Como $\alpha \equiv_{G} \beta$, existem $x, y \in G$ e uma $G$-forma $\lambda$ tais que

$$
\left\langle\alpha_{1}, x\right\rangle \equiv_{G}\left\langle\beta_{1}, y\right\rangle,\left\langle\alpha_{2}, \ldots, \alpha_{n}\right\rangle \equiv_{G}\langle x\rangle \oplus \lambda \text { e }\left\langle\beta_{2}, \ldots, \beta_{n}\right\rangle \equiv_{G}\langle y\rangle \oplus \lambda .
$$

Note que $\left\langle\beta_{1},-\alpha_{1}\right\rangle$ é uma $H$-forma. Como acima, as duas primeiras isometrias em $(3)$ e a condição (RS) implicam que existe

$$
u \in H \cap D_{G}\left(\beta_{1},-\alpha_{1}\right) \cap D_{G}\left(\left\langle\alpha_{2}, \ldots, \alpha_{n}\right\rangle\right),
$$

e portanto existe $v \in H$ e uma $G$-forma $\theta$ tais que

$$
\left\langle\alpha_{1}, u\right\rangle \equiv_{G}\left\langle\beta_{1}, v\right\rangle \text { e }\left\langle\alpha_{2}, \ldots, \alpha_{n}\right\rangle \equiv_{G}\langle u\rangle \oplus \theta .
$$

$$
\begin{aligned}
\operatorname{Logo}, \beta & \equiv_{G} \quad \alpha=\left\langle\alpha_{1}\right\rangle \oplus\left\langle\alpha_{2}, \ldots, \alpha_{n}\right\rangle \equiv_{G}\left\langle\alpha_{1}\right\rangle \oplus\langle u\rangle \oplus \theta=\left\langle\alpha_{1}, u\right\rangle \oplus \theta \\
& \equiv_{G}\left\langle\beta_{1}, v\right\rangle \oplus \theta,
\end{aligned}
$$

e a lei de cancelamento de Witt (3.9.(b)) implica que $\left\langle\beta_{2}, \ldots, \beta_{n}\right\rangle \equiv_{G}\langle v\rangle \oplus \theta$. Uma vez que $u, v \in H$, esta última isometria, a segunda isometria em (4) e o item (a) implicam que existe uma $H$-forma $\chi$ tal que

$$
\left\langle\alpha_{2}, \ldots, \alpha_{n}\right\rangle \equiv_{G}\langle u\rangle \oplus \chi \text { e }\left\langle\beta_{2}, \ldots, \beta_{n}\right\rangle \equiv_{G}\langle v\rangle \oplus \chi
$$

que juntamente com a primeira isometria em (4) completam a prova de (b) e do Fato 3.25.

Segue imediatamente do item (b) do Fato 3.25 que $H$ é um subgrupo completo de $G$. Sejam agora $\varphi, \psi H$-formas, tais que $\varphi \preceq_{G} \psi, \operatorname{com} \varphi=\left\langle a_{1}, \ldots, a_{n}\right\rangle, n \geq 1$. Então existe uma $G$-forma $\theta$ tal que

$$
\psi \equiv_{G} \varphi \oplus \theta=\left\langle a_{1}, \ldots, a_{n}\right\rangle \oplus \theta .
$$

Como $\psi$ é uma $H$-forma e $a_{1} \in H$, o item (b) da Fato 3.25 implica que existem $v_{1} \in H$ e uma $H$-forma $\chi_{1}$ tais que

$$
\left\langle a_{2}, \ldots, a_{n}\right\rangle \oplus \theta \equiv_{G}\left\langle v_{1}\right\rangle \oplus \chi_{1} .
$$

Uma vez que $\left\langle v_{1}\right\rangle \oplus \chi_{1}$ é uma $H$-forma e $a_{2} \in H$, outra aplicação de 3.25.(b) fornece $v_{2} \in H$ e uma $H$-forma $\chi_{2}$ tais que

$$
\left\langle a_{3}, \ldots, a_{n}\right\rangle \oplus \theta \equiv_{G}\left\langle v_{2}\right\rangle \oplus \chi_{2} .
$$

Após $n=\operatorname{dim} \varphi$ passos, obtemos $v_{n} \in H$ e uma $H$-forma $\chi_{n}$ tal que

$$
\theta \quad \equiv_{G}\left\langle v_{n}\right\rangle \oplus \chi_{n}=_{\text {def }} \quad \theta^{\prime} \text {. }
$$

Como $\theta^{\prime}$ é uma $H$-forma, $G$ - isométrica à $\theta$, concluímos que $\psi \equiv_{G} \varphi \oplus \theta^{\prime}$. Agora, o fato que $H$ é um subgrupo completo de $G$ acarreta $\psi \equiv_{H} \varphi \oplus \theta^{\prime}$, mostrando que $\varphi \preceq_{H} \psi$ e completando a prova do teorema 


\subsection{Subgrupos Saturados e Quocientes}

No Capítulo 2 de [DM1] há um estudo detalhado de subgrupos de Pfister e subgrupos saturados. Esta seção descreve apenas a estrutura básica que servirá como referência para o resto do trabalho.

Definição 3.26. Sejam $G$ um grupo especial e $\Delta$ um subgrupo de $G$.

a) Para $A \subseteq G$,

$\operatorname{Pfister}(A)=\left\{\psi: \psi\right.$ é uma G-forma e $\left.\psi \equiv_{G} \bigotimes_{i=1}^{n}\left\langle 1, a_{j}\right\rangle, \operatorname{com}\left\{a_{1}, \ldots, a_{n}\right\} \subseteq A\right\}$, é o conjunto das $G$-formas de Pfister com coeficientes em A (ou sobre A).

b) $\Delta$ é saturado se para todo $a \in \Delta, D_{G}(1, a) \subseteq \Delta$. Ssat $(G)$ indica o conjunto dos subgrupos saturados de $G$.

É fácil verificar que a interseção de qualquer família de subgrupos saturados de $G$ é saturado. Isto fornece o item (a) do seguinte

Lema 3.27. Sejam $G$ um grupo especial e $\Delta$ um subgrupo de $G$.

a) $\langle\operatorname{Ssat}(G), \subseteq\rangle$ é um reticulado completo, onde infimos são dados por interseções.

b) Para $A \subseteq G$ seja $[A]$ o subgrupo de $G$ gerado por $A$ e $A^{\text {sat }}$ a interseção de todos os subgrupos saturados de $G$ que contém $A$. Então

$$
A^{\text {sat }}=\bigcup\left\{D_{G}(\psi): \psi \text { é uma forma de Pfister com coeficientes em }[A]\right\} \text {, }
$$

é o subgrupo saturado de $G$ gerado por $A$.

c) $\Delta$ é saturado e $\psi$ é uma forma de Pfister com coeficientes em $\Delta$, então $D_{G}(\psi) \subseteq \Delta$.

d) Se $\psi$ é uma G-forma de Pfister e $G$ é reduzido então $D_{G}(\psi)$ é saturado.

e) Se $\psi_{1}, \psi_{2}$ são formas de Pfister sobre $G$, as seguintes condições são equivalentes :

(1) $D_{G}\left(\psi_{1}\right) \subseteq D_{G}\left(\psi_{2}\right)$;

(2) $S e \varphi$, $\theta$ são $G$-formas de mesma dimensão,

$$
\psi_{1} \otimes \varphi \equiv_{G} \psi_{1} \otimes \theta \Rightarrow \psi_{2} \otimes \varphi \equiv_{G} \psi_{2} \otimes \theta .
$$

f) Se $f: G \longrightarrow H$ é um SG-morfismo, a imagem inversa de um subgrupo saturado de $H$ é um subgrupo saturado de $G$.

Prova. Os itens (a) e (f) estão claros; os itens (b) e (c) estão provados na Prop. 2.6 em [DM1]; para (d) veja o Cor. 2.8.(a) em [DM1], enquanto que (e) vem do Lema 2.20 em [DM1]. 
Observação 3.28. Sejam $G, H$ grupos especiais e $G \stackrel{f}{\longrightarrow} H$ um SG-morfismo.

a) Por 3.27.(b), o menor subgrupo saturado de $G$ é

$$
\operatorname{Sat}(G)=_{\text {def }}\{1\}^{\text {sat }}=\bigcup\left\{D_{G}\left(2^{n}\right): n \geq 0\right\},
$$

onde $2^{n}=\underbrace{\langle 1,1\rangle \otimes \ldots \otimes\langle 1,1\rangle}_{n \text { vezes }}$, como em 3.5.(c). Assim, é fácil ver que

$$
G \text { é reduzido } \Leftrightarrow \operatorname{Sat}(G)=\{1\} .
$$

b) Do item (a) e de 3.27.(f) segue que se $H$ é reduzido, ker $f$ é um subgrupo saturado de $G$. Em relação a esta questão veja o Lema 3.30 abaixo.

Embora em [DM1] seja discutido o caso mais geral de quocientes por subgrupos de Pfister, iremos apresentar aqui quocientes por subgrupos saturados, os que serão utilizados no trabalho.

Se $\Delta$ é um subgrupo saturado de um grupo special $G$, seja

$$
G / \Delta=\{a / \Delta: a \in G\} \quad \text { e } \quad p_{\Delta}: G \longrightarrow G / \Delta
$$

o quociente usual de grupos abelianos e o morfismo quociente canônico $\left(p_{\Delta}(a)=a / \Delta\right)$. Definimos uma relação $\equiv_{G / \Delta}$ em $G / \Delta$ da seguinte forma

$$
\langle a / \Delta, b / \Delta\rangle \equiv_{G / \Delta}\langle c / \Delta, d / \Delta\rangle \quad \text { sse } \quad\left\{\begin{array}{c}
\text { Existe } \psi \in \text { Pfister }(\Delta) \text { tal que } \\
\psi \otimes\langle a, b\rangle \equiv_{G} \psi \otimes\langle c, d\rangle .
\end{array}\right.
$$

Das Proposições 2.21 e 2.28 em [DM1] obtemos o

Teorema 3.29. Seja $G$ um grupo especial e $\Delta$ um subgrupo saturado de $G$.

a) A relação $\equiv_{G / \Delta}$ está bem definida, $\left\langle G / \Delta, \equiv_{G / \Delta},-1 / \Delta\right\rangle$ é um grupo especial reduzido e a função quociente canônica, $p_{\Delta}$, é um $S G$-morfismo. Além disso, para $a, b, c, d \in G$,

$$
\langle a / \Delta, b / \Delta\rangle \equiv_{G / \Delta}\langle c / \Delta, d / \Delta\rangle \Leftrightarrow \Leftrightarrow^{9}\left\{\begin{array}{c}
\text { Existem } x, y, t, z \in G \text { tais que } \\
x a, y b, t c, d z \in \Delta e\langle x, y\rangle \equiv_{G}\langle t, z\rangle .
\end{array}\right.
$$

b) Se $\varphi, \theta$ são $G$-formas de mesma dimensão, as seguintes condições são equivalentes :

(1) $p_{\Delta} \star \varphi \equiv_{G / \Delta} p_{\Delta} \star \theta$;

(2) Existe $\psi \in \operatorname{Pfister}(\Delta)$ tal que $\psi \otimes \varphi \equiv_{G} \psi \otimes \theta$.

c) Se $f: G \longrightarrow H$ é um $S G$-morfismo tal que $\Delta \subseteq$ ker $f$, então existe um único $S G$-morfismo $\widehat{f}: G / \Delta \longrightarrow H$ que faz comutar o seguinte diagrama :

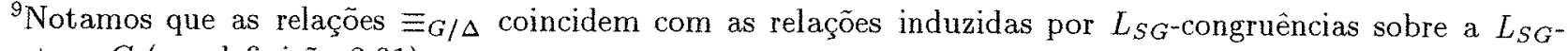
estrutura $G$ (ver definição 2.31).
} 


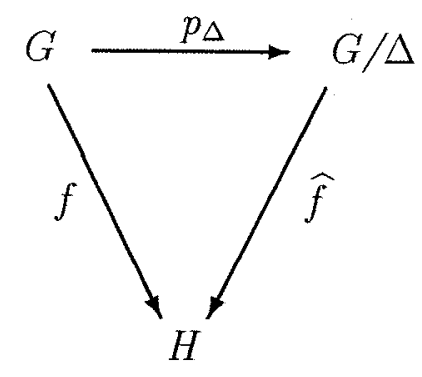

Lema 3.30. Sejam $G$ um grupo especial e $\Delta$ um subgrupo de $G$.

a) Os subgrupos saturados de $G$ são exatamente os núcleos dos $S G$-morfismos de $G$ em grupos especiais reduzidos.

b) $O$ subgrupos maximais saturados de $G$ são exatamente os núcleos dos $S G$-carácteres de $G$, isto é, os núcleos dos elementos de $X_{G}(3.18 .(a))$.

c) (Teorema da Separação) $\Delta^{\text {sat }}=\bigcap\left\{\operatorname{ker} \sigma: \sigma \in X_{G} e \Delta \subseteq \operatorname{ker} \sigma\right\}$.

Prova. Veja o Corolário 2.29 e o Teorema 2.11 em [DM1].

Há ainda um conceito de morfismo que será útil mais tarde :

Definição 3.31. Um $S G$-morfismo $f: G \longrightarrow H$ é regular se para todo $a, b \in G$, $f(a) \in D_{H}(1, f(b)) \Rightarrow \exists u, v \in G$ tal que $f(a)=f(u), f(b)=f(v)$ eu $u \in D_{G}(1, v)$.

Para SG-morfismos sobrejetores temos a seguinte caracterização de quocientes :

Proposição 3.32. Seja $f: G \longrightarrow H$ um SG-morfismo sobrejetor. As seguintes condiçôes sãa equivalentes :

(1) $f$ é regular.

(2) O SG-morfismo $\widehat{f}: G / \operatorname{ker} f \longrightarrow H$, induzido por $f$, é um $S G$-isomorfismo.

Prova. Ver Proposição 2.23 em [DM1].

Exemplo 3.33. Os seguintes são exemplos de SG-morfismos regulares :

(1) Se $G$ é um grupo especial e $\Delta$ é um subgrupo saturado de $G$, então o SG-morfismo quociente, $p_{\Delta}: G \longrightarrow G / \Delta$, é regular. De fato, por 3.29.(a), dados $a, b \in G$ tais que $a / \Delta \in D_{G / \Delta}(1, b / \Delta)$, existem $x, y \in \Delta$ tais que 


$$
x a \in D_{G}(1, y b) .
$$

Mas então, $p_{\Delta}(a x)=p_{\Delta}(a)$ e $p_{\Delta}(b y)=p_{\Delta}(b)$, conforme necessário.

Como aplicação temos o seguinte fato, que será utilizado muitas vezes no que segue :

Fato 3.34. G um grupo especial e $\Delta$ um subgrupo saturado de $G$. Se $\Sigma$ é um sugrupo saturado de $G$ tal que $\Delta \subseteq \Sigma$, então $p_{\Delta}(\Sigma)=\Sigma / \Delta$ é um subgrupo saturado de $G / \Delta$.

Prova. Suponha que $b / \Delta \in \Sigma / \Delta$ e que $a / \Delta \in D_{G / \Delta}(1, b / \Delta)$. Logo, existem $x, y \in \Delta$ tais que

$$
x a \in D_{G}(1, y b) \text {. }
$$

Uma vez que $b \in \Sigma$ e $y \in \Delta \subseteq \Sigma$, temos que $b y \in \Sigma$. A saturação de $\Sigma$ acarreta $a x \in \Sigma$, e portanto, $a / \Delta=a x / \Delta \in \Sigma / \Delta$, estabelecendo a saturação de $\Sigma / \Delta$.

(2) Seja $G_{i}, i \in I$, uma família não vazia em RSG e $G$ o produto dos $G_{i}$. Cada projeção $\pi_{i}: G \longrightarrow G_{i}$ é um SG-morfismo regular.

(3) Suponha que $G^{\prime} \stackrel{j}{\longrightarrow} G \stackrel{p}{\longrightarrow} G^{\prime}$ são SG-morfismos tais que $p \circ j=I d_{G^{\prime}}$. Então, o SG-morfismo $p: G \longrightarrow G^{\prime}$ é regular.

O restante desta seção, que é original, é destinada a mostrar que a equivalência no item (b) do Teorema 3.29 pode ser estendida às formulas existenciais positivas (2.4) da linguagem $L_{S G}$. Embora a construção seja possível para quocientes por subgrupos de Pfister, iremos nos restringir a quocientes por subgrupos saturados.

Fixado um grupo especial $H$, seja $L_{S G}(H)$ a linguagem de primeira ordem obtida a partir da linguagem dos grupos especiais, $L_{S G}$ (3.14), adicionando-se o seguinte conjunto de novas constantes,

$$
\underline{H}=\{\underline{x}: x \in H \backslash\{1,-1\}\} .
$$

Está claro que $H$ é naturalmente uma $L_{S G}(H)$-estrutura. Lembramos que se $L$ é uma linguagem de primeira ordem, $p p(L)$ indica a coleção das fórmulas positivas primitivas em $L$ e $\exists^{+}(L)$ indica a família das fórmulas existenciais positivas em $L$.

Se $\alpha, \beta$ são formas de mesma dimensão sobre $H$, a definição de isometria em $H$ (item (C) em 3.1) mostra que a relação $\alpha \equiv_{H} \beta$ pode ser descrita por uma fórmula ${ }^{10}$ em $p p\left(L_{S G}(H)\right)$. Vamos utilizar este fato na definição que segue.

Definição 3.35. Gejam $H$ um grupo especial e $\psi$ uma forma de Pfister sobre $H$. Por indução na complexidade, definimos uma função

$$
(\cdot)^{\psi}: \exists^{+}\left(L_{S G}\right) \longrightarrow \exists^{+}\left(L_{S G}(H)\right), \varphi \longmapsto \varphi^{\psi},
$$

\footnotetext{
${ }^{10}$ Eventualmente, muito longa.
} 
dada por, onde $r_{0}, r_{1}, s_{0}, s_{1}$, são termos em $L_{S G}$ e $\bar{v}=\left\langle v_{1}, \ldots, v_{n}\right\rangle$ são variáveis :

(i) $\left(r_{0}(\bar{v})=r_{1}(\bar{v})\right)^{\psi}$ é a conjunção de fórmulas em $p p\left(L_{S G}(H)\right)$ que descreve a relação

$$
r_{0}(\bar{v}) \psi \equiv_{H} r_{1}(\bar{v}) \psi
$$

(ii) $\left(\left\langle r_{0}(\bar{v}), s_{0}(\bar{v})\right\rangle \equiv\left\langle r_{1}(\bar{v}), s_{1}(\bar{v})\right\rangle\right)^{\psi}$ é a conjunção de fórmulas em $p p\left(L_{S G}(H)\right)$ que descreve a relação

$$
\left\langle r_{0}(\bar{v}), s_{0}(\bar{v})\right\rangle \otimes \psi \quad \equiv_{H} \quad\left\langle r_{1}(\bar{v}), s_{1}(\bar{v})\right\rangle \otimes \psi
$$

(iii) $\left(\varphi_{1} \wedge \varphi_{2}\right)^{\psi}=\varphi_{1}^{\psi} \wedge \varphi_{2}^{\psi}$;

(iv) $\left(\varphi_{1} \vee \varphi_{2}\right)^{\psi}=\varphi_{1}^{\psi} \vee \varphi_{2}^{\psi}$;

$(v)(\exists x \varphi)^{\psi}=\exists x \varphi^{\psi}$.

Está claro que a associação $\varphi \longmapsto \varphi^{\psi}$ preserva as variáveis livres e/ou ligadas de $\varphi$.

Proposição 3.36. Sejam $\varphi\left(v_{1}, \ldots, v_{n}\right) \in \exists^{+}\left(L_{S G}\right)$ e $H$ um grupo especial. Se $P$, $Q$ são formas de Pfister sobre $H$ tais que $D_{H}(P) \subseteq D_{H}(Q)$ e $\bar{z} \in H^{n}$, então

$$
H \vDash \varphi^{P}[\bar{z}] \quad \Rightarrow \quad H \vDash \varphi^{Q}[\bar{z}] \text {. }
$$

Prova. Por indução na complexidade; se $\varphi$ é atômica a conclusão segue de 3.27.(e). O passo de indução para a conjunção e a disjunção são imediatos. Para o quantificador $\exists$, temos

$$
\begin{aligned}
H \vDash(\exists x \phi(x ; \bar{v}))^{P}[\bar{z}] & \Leftrightarrow H \vDash \exists x \varphi^{P}(x ; \bar{v})[\bar{z}] \Leftrightarrow \exists a \in H \text { tal que } H \models \varphi^{P}[a, \bar{z}] \Rightarrow \\
& \Rightarrow \exists a \in H \text { tal que } H \models \varphi^{Q}[a, \bar{z}] \Leftrightarrow H \vDash(\exists x \varphi(x ; \bar{v}))^{Q}[\bar{z}],
\end{aligned}
$$

completando a indução.

O resultado que segue fornece um critério para que uma fórmula existencial positiva de $L_{S G}$ com parâmetros em um grupo especial seja válida em um quociente por um subgrupo saturado.

Proposição 3.37. Sejam $H$ um grupo especial e $\Delta$ um subgrupo saturado de $H$. Para cada $\varphi\left(v_{1}, \ldots, v_{n}\right)$ em $\exists^{+}\left(L_{S G}\right)$ e $\bar{z} \in H^{n}$, as seguintes condiçôes são equivalentes :

(1) $H / \Delta \models \varphi[\bar{z} / \Delta]{ }^{11}$

(2) Existe uma forma de Pfister $P$ sobre $\Delta$ tal que $H \models \varphi^{P}[\bar{z}]$.

Prova. Começamos com o seguinte

Fato 3.38. (1) e (2) são equivalentes para fórmulas atômicas.

\footnotetext{
${ }^{11} \bar{z} / \Delta=\left\langle z_{1} / \Delta, \ldots, z_{n} / \Delta\right\rangle$.
} 
Prova. Sejam $r_{0}(\bar{v}), s_{0}(\bar{v}), r_{1}(\bar{v})$ e $s_{1}(\bar{v})$ termos em $L_{S G}$.

Para as fórmulas $r_{0}=r_{1}$ e $\left\langle r_{0}, s_{0}\right\rangle \equiv_{G}\left\langle r_{1}, s_{1}\right\rangle$ a equivalência entre (1) e (2) é consequência da equivalência no item (b) do Teorema $3.29^{12}$. Apresentamos porém uma verificação mais explícita no caso $r_{0}=r_{1}$ :

Fixado $\bar{z} \in H^{n}$, sejam $x=r_{0}(\bar{z})$ e $y=r_{1}(\bar{z})$ os elementos correspondentes de $H$. Então,

$$
x / \Delta=y / \Delta \Leftrightarrow x y \in \Delta
$$

Seja $P=\langle 1, x y\rangle$; então $P$ é uma forma de Pfister sobre $\Delta$ e temos

$$
x\langle 1, x y\rangle \equiv_{H}\langle x, y\rangle \equiv_{H} y\langle 1, x y\rangle
$$

mostrando que $H \models(x=y)^{P}$ e que $(1) \Rightarrow(2)$ para a fórmula $r_{0}=r_{1}$. Reciprocamente, suponha que existe uma forma de Pfister $Q$ sobre $\Delta$ tal que $H \models\left(r_{0}=r_{1}\right)^{Q}[\bar{z}]$. Isto significa que em $H$ temos (utilizando a mesma notação que acima)

$$
x Q \equiv_{H} y Q .
$$

Assim, $x y Q \equiv_{H} Q$ e portanto 3.9.(f) garante que $x y \in D_{H}(Q)$. Como $\Delta$ é saturado, 3.27.(c) implica que $x y \in \Delta$. Mas então, $x / \Delta=y / \Delta$, isto é, $H / \Delta \models\left(r_{0}=r_{1}\right)[\bar{z} / \Delta]$, concluindo a verificação.

Iremos agora mostrar sucessivamente $(1) \Rightarrow(2)$ e $(2) \Rightarrow(1)$ por indução na complexidade das fórmulas em $\exists^{+}\left(L_{S G}\right)$.

$(1) \Rightarrow(2)$ : Para a conjunção temos

$$
H / \Delta \models \varphi \wedge \psi[\bar{z} / \Delta] \Leftrightarrow H / \Delta \vDash \varphi[\bar{z} / \Delta] \text { e } H / \Delta \models \psi[\bar{z} / \Delta]
$$

Pela hipótese de indução, existem formas de Pfister $P, Q$ sobre $\Delta$ tais que

$$
H \vDash \varphi^{P}[\bar{z}] \text { e } H \models \psi^{Q}[\bar{z}] .
$$

Seja $R=P \otimes Q$; por 3.9.(g) $D_{H}(P), D_{H}(Q) \subseteq D_{H}(R)$ e $R$ é uma forma de Pfister sobre $\Delta$. Portanto, 3.36 acarreta

$$
H \vDash \varphi^{R}[\bar{z}] \text { e } H \models \psi^{R}[\bar{z}]
$$

Como $(\varphi \wedge \psi)^{R}=\varphi^{R} \wedge \psi^{R}$, obtemos $H \models(\varphi \wedge \psi)^{R}[\bar{z}]$, como necessário. O passo de indução para a conjunção é análogo (na realidade mais simples). Agora suponha que temos

$$
H / \Delta \vDash \exists x \varphi(x ; \bar{v})[\bar{z} / \Delta] \text {. }
$$

Então, existe $a \in H$ tal que $H / \Delta \models \varphi[a / \Delta ; \bar{z} / \Delta]$. A hipótese de indução fornece uma forma de Pfister $P$ sobre $\Delta$ tal que

$$
H \vDash \varphi^{P}[a ; \bar{z}] .
$$

Uma vez que $(\exists x \varphi)^{P}=\exists x \varphi^{P}$, a relação acima garante que $H \vDash(\exists x \varphi)^{P}[\bar{z}]$, como desejado.

\footnotetext{
${ }^{12}$ Se $H$ é reduzido, $s_{0}=s_{1}$ é equivalente a $\left\langle 1, s_{0}\right\rangle \equiv_{H}\left\langle 1, s_{1}\right\rangle$, e bastaria considerar 2-isometrias apenas.
} 


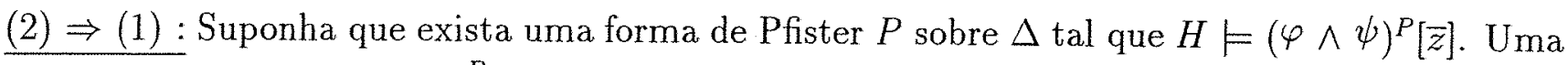
vez que $(\varphi \wedge \psi)^{P}=\varphi^{P} \wedge \psi^{P}$, obtemos, utilizando a hipótese de indução, $H \vDash \varphi^{P}[\bar{z}]$ e $H \vDash \psi^{P}[\bar{z}] \Rightarrow H / \Delta \models \varphi[\bar{z} / \Delta]$ e $H / \Delta \models \psi[\bar{z} / \Delta] \Leftrightarrow H / \Delta \models \varphi \wedge \psi[\bar{z} / \Delta]$, como desejado. O passo para disjunção é imediato. Finalmente, suponha que existe uma forma de Pfister $P$ sobre $\Delta$ tal que $H \models(\exists x \varphi(x ; \bar{v}))^{P}[\bar{z}]$. Então, existe $a \in H$ tal que

$$
H \vDash \varphi^{P}[a ; \bar{z}] .
$$

A hipótese de indução garante que $H / \Delta \vDash \varphi[a / \Delta ; \bar{z} / \Delta]$, i.e., $H / \Delta \models \exists x \varphi(x ; \bar{v})[\bar{z} / \Delta]$, completando a prova.

\subsection{Alguns Tipos de Grupos Especiais}

Nesta seção registraremos algumas construções que exemplificam o conceito de grupo especial.

\subsubsection{Produtos, Extensões e Fans}

3.39. Produtos : A categoria dos grupos especiais (respec. grupos especiais redüzidos) é fechada por produtos na categoria $\mathbf{L}_{\mathbf{S G}}-\bmod ^{13}$. Mais explicitamente:

Seja $G_{i}, i \in I$, uma família de grupos especiais e $G=\prod_{i \in I} G_{i}$ o grupo produto com a estrutura definida componente a componente. Para $i \in I$, seja

$$
\pi_{i}: G \longrightarrow G_{i}
$$

a projeção canônica na $i^{e ́ s i m a}$ coordenada. Indicamos por $1 \mathrm{e}-1 \in G$ as sequências constantes de valor $1 \mathrm{e}-1$, respectivamente. Para $u, v, x, y \in Q$, definimos

$$
\langle u, v\rangle \equiv_{G}\langle x, y\rangle \quad \Leftrightarrow \quad \forall i \in I,\left\langle u_{i}, v_{i}\right\rangle \equiv_{G_{i}}\left\langle x_{i}, y_{i}\right\rangle
$$

Então, $\left\langle G, \equiv_{G},-1\right\rangle$ é um grupo especial, que é reduzido se cada componente for reduzida. Além disso, as projeções $\pi_{i}$ são SG-morfismos. Esta construção é o produto da família $G_{i}$ na categoria dos grupos especiais. Em particular, 11 o grupo especial (reduzido) com um único elemento, é o objeto final (produto da família vazia de grupos especiais) na categoria dos grupos especiais (respec. grupos especiais reduzidos).

Se $H$ é um grupo especial e $I \neq \emptyset$ é um conjunto, com a estrutura descrita acima, $H^{I}$ é um grupo especial e a aplicação diagonal

$$
\delta: H \longrightarrow H^{I} \text {, onde } \delta(h) \text { é a sequência constante de valor } h,
$$

é um morfismo puro de grupos especiais, pois $\pi_{i} \circ \delta=I d_{H}$, onde $i$ é qualquer elemento de $I$. Assim $H$ é um retrato de qualquer suas potências por um conjunto não vazio.

${ }^{13} \mathrm{~A}$ construção dos produtos em categorias L-mod é realizada em 2.20 . 
3.40. Extensões : (Exemplo 1.10, [DM1]) Seja $G$ um grupo especial e $\Delta$ um grupo de expoente 2. Indicamos por $G[\Delta]$ o grupo cujo domínio é o produto $G \times \Delta$, com os elementos distinguidos $1=\langle 1,1\rangle \mathrm{e}-1=\langle-1,1\rangle$. Escrevemos $g \cdot \delta$, no lugar de $\langle g, \delta\rangle$, para um elemento de $G[\Delta]$. Para cada $g \cdot \delta \in G[\Delta]$ definimos um subgrupo $E_{g \cdot \delta}$ de $G[\Delta]$ da seguinte forma :

$$
E_{g \cdot \delta}= \begin{cases}D_{G}(1, g) \times 1 & \text { se } g \neq-1 \text { e } \delta=1 \\ G[\Delta] & \text { se } g=-1 \text { e } \delta=1 \\ \{1, g \cdot \delta\} & \text { se } \delta \neq 1\end{cases}
$$

Agora definimos uma relação $\equiv_{e x t}$ em $G[\Delta] \times G[\Delta]$ por

$$
\left\langle g_{1} \cdot \delta_{1}, g_{2} \cdot \delta_{2}\right\rangle \equiv_{e x t}\left\langle g_{3} \cdot \delta_{3}, g_{4} \cdot \delta_{4}\right\rangle \Leftrightarrow\left\{\begin{array}{rll}
g_{1} g_{2}=g_{3} g_{4} & \mathrm{e} & \delta_{1} \delta_{2}=\delta_{3} \delta_{4} \\
& \mathrm{e} \\
g_{3} g_{1} \cdot \delta_{3} \delta_{1} & \in E_{g_{1} g_{2} \cdot \delta_{1} \delta_{2}} .
\end{array}\right.
$$

Então, $\left\langle G[\Delta], \equiv_{e x t},\langle-1,1\rangle\right\rangle$ é um grupo especial, que é reduzido se $G$ o for. Esta construção denomina-se extensão de $G$ por $\Delta$. Note que temos SG-morfismos naturais

$$
G \stackrel{\iota}{\longrightarrow} G[\Delta] \stackrel{p}{\longrightarrow} G
$$

onde $\iota(g)=\langle g, 1\rangle$ e $p(g, \delta)=g$. Assim $p \circ \iota=I d_{G}$ e $G$ é um retrato de qualquer uma de suas extensões. Em particular, $\iota$ é mais um exemplo de um SG-morfismo puro.

3.41. Fans : (Exemplo 1.7, [DM1]) Sejam $G$ um grupo de expoente 2, com card $G \geq 2$, e -1 um elemento de $G$, distinto de 1. Para $-1 \neq a \in G$, seja $G_{a}=\{1, a\}$, com $G_{-1}=G$. Para $a, b, c, d \in G$, definimos

$$
\langle a, b\rangle \equiv_{f a n}\langle c, d\rangle \quad \Leftrightarrow \quad a b=c d \quad \text { e } \quad a c \in G_{c d} .
$$

Então, $\left\langle G, \equiv_{f a n},-1\right\rangle$ é um grupo especial reduzido, denominado fan de base $G$ e elemento distinguido -1. É fácil verificar que para todo $a \in G$ temos

$$
D_{G}(1, a)=\left\{\begin{array}{cc}
\{1, a\} & \text { se } a \neq-1 \\
G & \text { se } a=-1
\end{array}\right.
$$

É imediato que a estrutura de grupo especial reduzido dada a $\mathbb{Z}_{2}$ em 3.13 é exatamente a de fan de base $\mathbb{Z}_{2}=\{1,-1\}$ e que todo fan é isomorfo a alguma extensão de $\mathbb{Z}_{2}$ por um grupo de expoente 2 .

Um grupo especial $G$ é um fan sse satisfaz a seguinte condição ${ }^{14}$, para todo $u, v, x, y \in G$ :

* $1 \neq-1$;

$*\langle u, v\rangle \equiv\langle x, y\rangle \quad \Leftrightarrow \quad(u v=x y=-1)$ ou $(x y=u v \neq-1$ e $\{u, v\}=\{x, y\})$.

Assim, a noção de fan é defínivel por uma fórmula de primeira ordem em $L_{S G}$.

\footnotetext{
${ }^{14}$ Que é equivalente àquelas em $11.1 \mathrm{em}$ [DM1].
} 


\subsubsection{Corpos e Grupos Especiais}

Na seção 3 do Capítulo 1 de [DM1] o leitor encontrará uma discussão extensa sobre as relações entre grupos especiais e corpos. Faremos aqui apenas algumas indicações básicas, que motivarão a Definição 3.43 .

Seja $F$ um corpo, sempre de característica $\neq 2^{15}$. A seguinte notação será de uso constante:

$* \dot{F}=\{x \in F: x \neq 0\}$, o grupo multiplicativo de $F$;

$* F^{2}=\left\{x^{2}: x \in F\right\} ; \quad * \dot{F}^{2}=\left\{x^{2}: x \in \dot{F}\right\}$;

$* \Sigma F^{2}=\left\{\sum_{i \in I} x_{i}^{2}: I\right.$ é finito e $\left.x_{i} \in F\right\} ; \quad * \Sigma \dot{F}^{2}=\left\{\sum_{i \in I} x_{i}^{2}: I\right.$ é finito e $\left.x_{i} \in \dot{F}\right\}$.

Está claro que $\dot{F}^{2}=\dot{F} \cap F^{2}$ e é um subgrupo do grupo multiplicativo $\dot{F}$. Temos também $\dot{F} \cap \Sigma F^{2} \subseteq \Sigma \dot{F}^{2}$ e $\dot{F} \cap \Sigma F^{2}$ é um subgrupo de $\dot{F}$ : se $p=\sum_{i \in I} x_{i}^{2} \neq 0$ está em $\dot{F} \cap \Sigma F^{2}$ então

$$
\frac{1}{p}=\sum_{i \in I}\left(x_{i} / p\right)^{2}
$$

está em $\dot{F} \cap \Sigma F^{2}$.

Lembramos que um corpo é

* Pitagórico se $F^{2}=\Sigma F^{2}$.

* Formalmente real se $-1 \notin \Sigma \dot{F}^{2}$.

Note que um corpo é pitagórico sse toda soma de dois quadrados do corpo é um quadrado do corpo. Um corpo é formalmente real sse $\Sigma \dot{F}^{2}=\dot{F} \cap \Sigma F^{2}{ }^{16}$

Temos que todo corpo algebricamente fechado, como o corpo dos números complexos, é Pitagórico mas não formalmente real. Todo corpo real fechado, como o corpo dos números reais, é Pitagórico e formalmente real.

Para $T \subseteq \dot{F}$, seja $T^{*}=T \cup\{0\}$. Para $a, b \in \dot{F}$, definimos $\left[D_{T}\right]$

$$
D_{T}(a, b)=\left\{t \in \dot{F}: \exists p, q \in T^{*} \text { tais que } t=a p+b q\right\}
$$

Se $T$ é um subgrupo de $\dot{F}$, indicamos por $a_{T} \in \dot{F} / T$ o elemento do grupo quociente correspondente a $a \in \dot{F}$.

Para $T=\dot{F}^{2}$ ou $T=\dot{F} \cap \Sigma F^{2}$ definimos: para $a, b, c, d \in \dot{F}$ :

$\left[\equiv_{T}\right] \quad\left\langle a_{T}, b_{T}\right\rangle \equiv_{T}\left\langle c_{T}, d_{T}\right\rangle \Leftrightarrow a_{T} b_{T}=c_{T} d_{T} \quad$ e $\quad D_{T}(a, b)=D_{T}(c, d)$.

Com estes preliminares, enunciamos a seguinte consequência do Teorema 1.32 em [DM1] :

Proposição 3.42. Seja $F$ um corpo de característica $\neq 2$. Com a notação acima :

\footnotetext{
${ }^{15}$ Equivalentemente, $2=1+1$ é invertivel em $F$.

${ }^{16}$ Assim, se $F$ é formalmente real, $\Sigma \dot{F}^{2}$ é um subgrupo de $\dot{F}$.
} 
a) Se $T=\dot{F}^{2}, \mathcal{G}(F)=_{\text {def }}\left\langle\dot{F} / T, \equiv_{T},-1 / T\right\rangle$ é um grupo especial.

b) $S e T=\dot{F} \cap \Sigma F^{2}, \mathcal{G}_{r e d}(F)=_{\text {def }}\left\langle\dot{F} / T, \equiv_{T},-1 / T\right\rangle$ é um grupo especial reduzido e a aplicação

$$
a_{\dot{F}^{2}} \in \mathcal{G}(F) \longmapsto a_{T} \in \mathcal{G}_{r e d}(F)
$$

é um morfismo de grupos especiais.

c) $\mathcal{G}(F)$ é reduzido sse $F$ é Pitagórico.

Note que se $F$ é um corpo Pitagórico temos $\mathcal{G}_{\text {red }}(F)=\mathcal{G}(F)$. Se $F$ é um corpo formalmente real então card $\mathcal{G}_{\text {red }}(F) \geq 2$.

Registramos que se $T$ é uma pré-ordem em $F$ então $\quad \mathcal{G}_{T}(F)=_{\text {def }}\left\langle\dot{F} / T, \equiv_{T},-1 / T\right\rangle \quad$ é um grupo especial reduzido, generalizando o item (b) em 3.42 .

Se $F, K$ são corpos e $f: F \longrightarrow K$ é um morfismo de anéis que preserva unidades então $f$ induz um SG-morfismo natural

$$
\mathcal{G}(f): \mathcal{G}(F) \longrightarrow \mathcal{G}(K)
$$

que leva a classe quadrática de um elemento de $a \in \dot{F}$ na classe quadrática de $f(a)$ em $\dot{K}$. Deste modo, $\mathcal{G}$ torna-se um funtor, $\mathcal{G}: \operatorname{Corp}_{\mathbf{1} / 2} \longrightarrow \mathrm{SG}$. De modo análogo podemos definir o funtor $\mathcal{G}_{\text {red }}:$ Corp $_{1 / 2} \longrightarrow$ RSG $\cdot{ }^{17}$

Definição 3.43. Um grupo especial $G$ é representável se existe um corpo $F$ de característica $\neq 2$ tal que $G$ é isomorfo a $\mathcal{G}(F)$.

Se $G$ é reduzido então, pelo item (c) da Proposição 3.42 acima, $G$ é representável sse existe um corpo Pitagórico $F$ tal que $G$ é isomorfo a $\mathcal{G}(F)$.

Em relação à representabilidade temos a seguinte Proposição, cuja prova omitiremos :

Proposição 3.44. Todo ultraproduto de grupos especiais representáveis é representável.

Um problema aberto importante, enunciado por M. Marshall, pergunta se todo grupo especial reduzido é representável ${ }^{18}$.

Um resultado bem conhecido devido a A. Pfister pode ser enunciado, na linguagem dos grupos especiais, da seguinte forma :

\footnotetext{
${ }^{17} \operatorname{Corp}_{1 / 2}$ designa a categoria dos corpos onde $2=1+1$ é invertível e morfismos de anéis que preservam unidade.

${ }^{18} \mathrm{~A}$ maioria dos que trabalham na área acredita que a resposta é negativa.
} 
Teorema 3.45. Sejam $F$ um corpo $e \varphi, \psi$ formas diagonais sobre $\dot{F}$, com $\psi$ anisotrópica. As seguintes condições são equivalentes :

(1) $\varphi \preceq \mathcal{G ( F )} \psi$;

(2) Se $F \stackrel{\iota}{\longrightarrow} K$ é uma extensão de $F$, então $D_{\mathcal{G}(K)}(\mathcal{G}(\iota) \star \varphi) \subseteq D_{\mathcal{G}(K)}(\mathcal{G}(\iota) \star \psi)$.

(3) Se $n=\operatorname{dim} \varphi$ e $F \stackrel{\iota}{\longrightarrow} F\left(x_{1}, \ldots, x_{n}\right)=K$ é a imersão canônica, então $D_{\mathcal{G}(K)}(\mathcal{G}(\iota) \star \varphi) \subseteq D_{\mathcal{G}(K)}(\mathcal{G}(\iota) \star \psi)$.

(4) Se $n=\operatorname{dim} \varphi$ e $F \stackrel{\iota}{\longrightarrow} F\left(x_{1}, \ldots, x_{n}\right)=K$ é a imersão canônica, então $\varphi\left(x_{1}, \ldots, x_{n}\right) \in D_{\mathcal{G}(K)}(\mathcal{G}(\iota) \star \psi)$.

\subsection{3 Álgebras de Boole e Grupos Especiais}

Assumimas que o leitor tem familiaridade com a teoria das álgebras de Boole. Uma referência para o assunto é [BD]. Nos Capítulos 4 e 7 de [DM1] há um estudo extenso da interação entre álgebras de Boole e grupos especiais. Como no caso dos corpos, apresentaremos aqui apenas algumas das noções básicas acerca do tema.

Se $B$ é uma álgebra de Boole e $a, b \in B$, indicamos por

$$
a \wedge b, a \vee b \text { e }-a
$$

o ínfimo, o supremo de $a, b$ e o complemento $a$, respectivamente. Sejam

$$
\perp=\text { mínimo de } B \text { e } ' T=\text { máximo de } B \text {. }
$$

Se $S \subseteq B, \wedge S$ e $\bigvee S$ indicam o ínfimo e o supremo de $S$ em $B$ (se existirem), respectivamente.

Recordamos a seguinte

Definição 3.46. Sejam $B$ uma álgebra de Boole $e \emptyset \neq V \subseteq B$.

a) $V$ é um filtro se for fechado por inf's finitos e para todo $a, b \in B$

$$
a \in V \quad e \quad a \leq b \Rightarrow b \in V .
$$

b) $V$ é um ideal se for fechado em relação a sup's finitos e para todo $a, b \in B$

$$
b \in V \quad e \quad a \leq b \quad \Rightarrow \quad a \in V .
$$

Filtros próprios ${ }^{19}$ maximais pela ordem parcial da inclusão são denominados ultrafiltros. Analogamente, temos o conceito de ideal maximal.

Se $A, B$ são álgebras de Boole, um Ba-morfismo, $f: A \longrightarrow B$, é uma função que preserva $\perp, T, \wedge$ e $\vee$. Indicamos por $\mathbf{B a}$ a categoria das álgebras de Boole e seus morfismos.

\footnotetext{
${ }^{19}$ Isto é, distintos de $A$.
} 
Para $a, b \in B$, a operação

$$
a \Delta b={ }_{\text {def }}(a \wedge-b) \vee(b \wedge-a)
$$

denomina-se a diferença simétrica de $a$ e $b$. É bem conhecido que

(I) $\langle B, \Delta, \perp\rangle$ é um grupo de expoente 2, com elemento neutro $\perp$;

(II) Para $a, b \in B$

$$
\text { (i) }-a=\top \Delta a ; \quad \text { (ii) } a \vee b=a \Delta b \Delta(a \wedge b) .
$$

(III) $\langle B, \Delta, \wedge, \perp, T\rangle$ é um anel comutativo, com $\Delta$ no papel de soma e $\wedge$ no papel de produto, cujos neutros são respectivamente $\perp$ e $T$.

Para $a, b, c, d \in B$, definimos

$$
\left[\equiv_{B}\right] \quad\langle a, b\rangle \equiv_{B}\langle c, d\rangle \quad \Leftrightarrow \quad a \wedge b=c \wedge d \text { e } a \vee b=c \vee d \text {. }
$$

Observe que no caso de álgebras de Boole temos $1=\perp$.

Proposição 3.47. Com a notação acima $e-1=\top$ temos:

a) A tripla $\left\langle B, \equiv_{B},-1\right\rangle$ é um grupo especial reduzido tal que para $a, b \in B$

$$
a \in D_{B}(1, b) \Leftrightarrow a \leq b .
$$

b) Se $A, B$ são álgebras de Boole e $f: A \longrightarrow B$ é uma função, então

$$
f \text { é um SG-morfismo } \Leftrightarrow \quad f \text { é um Ba-morfismo. }
$$

c) O subgrupos saturados de $B$ são exatamente os ideais de $B$.

Prova. Para (a), veja Corolário 4.4.(b), para (b) a Proposição 4.5 e para (c) a Proposição 4.7.(a) em [DM1].

Nos Capítulos 4, 5 e 7 de [DM1] mostra-se que as álgebras de Boole têm ligações importantes com os grupos especiais reduzidos. Uma destas construções é a envoltória Booleana, que passaremos a descrever.

Seja $G$ um grupo especial reduzido e $B_{G}$ a álgebra de Boole dos abertos e fechados de seu espaço de ordens (3.18). Definimos uma aplicação

$\varepsilon_{G}: G \longrightarrow B_{G}$, dada por $\varepsilon_{G}(a)=[a=-1]=\left\{\sigma \in X_{G}: \sigma(a)=-1\right\}=[-a=1]$. O diagrama $\varepsilon_{G}: G \longrightarrow B_{G}$ denomina-se a envoltória Booleana de $G$. As principais propriedades desta construção constam do

Teorema 3.48. Seja $G$ um grupo especial reduzido. Com a notação acima

a) $\varepsilon_{G}$ é uma imersão completa.

b) Para cada $u \in B_{G}$, existe uma família $\left\{F_{1}, \ldots, F_{n}\right\}$ de subconjuntos finitos de $G$ tal que

$$
u=\bigcup_{i=1}^{n} \bigcap_{a \in F_{i}} \varepsilon_{G}(a) .
$$


c) Para todo $\sigma \in X_{G}$ existe um único $\sigma_{*} \in X_{B_{G}}$ tal que $\sigma_{*} \circ \varepsilon_{G}=\sigma$, isto é, o triângulo abaixo é comutativo:
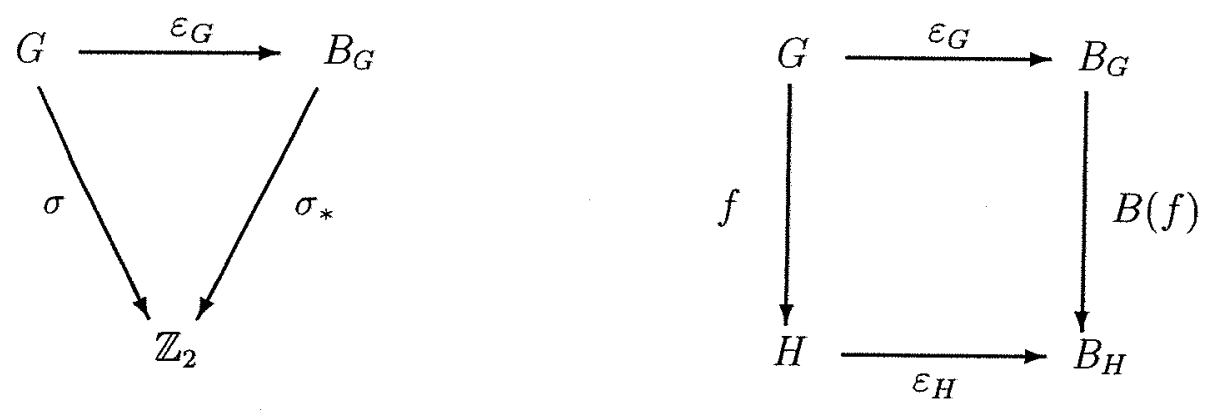

d) Se $P=\bigotimes_{i=1}^{n}\left\langle 1, a_{i}\right\rangle$ é uma forma de Pfister sobre $G$ e $a \in G$, então

$$
a \in D_{G}(P) \Leftrightarrow \varepsilon_{G}(a) \subseteq \bigcup_{i=1}^{n} \varepsilon_{G}\left(a_{i}\right) .
$$

e) Se $f: G \longrightarrow H$ é um SG-morfismo de grupos reduzidos, existe um único Ba-morfismo, $B(f): B_{G} \longrightarrow B_{H}$, que faz o quadrado acima comutativo. As associações

$$
G \longmapsto B_{G} \quad \text { e } \quad(G \stackrel{f}{\longrightarrow} H) \longmapsto\left(B_{G} \stackrel{B(f)}{\longrightarrow} B_{H}\right)
$$

determinam um funtor, $B: \mathbf{R S G} \longrightarrow \mathbf{B a}$, que é adjunto à esquerda do funtor esquecimento de Ba para $\mathbf{R S G}$. ${ }^{20}$

Prova. a) O item (a) vem do Corolário 5.4, o item (b) da Proposição 4.10.(b) e o item (c) é equivalente ao item (a) pelo Teorema 5.2; (d) vem da Proposição 4.11 e (e) do Teorema 4.17, todos em [DM1].

\subsubsection{Grupos Especiais de Comprimento de Cadeia Finito}

Em [Ast1] e [Ast3] há um estudo aprofundado dos grupos especiais do título. Alguns destes resultados serão importantes no Capítulo 5, quando apresentarmos a envoltória profinita de um grupo especial reduzido. Exceto pelo Corolário 3.52, todos os resultados e definições desta subseção aparecem em [Ast1].

Definição 3.49. Seja G um grupo especial.

a) Uma cadeia em G é uma sequência estritamente crescente

$$
D_{G}\left(1, a_{0}\right) \subsetneq D_{G}\left(1, a_{1}\right) \subsetneq \ldots \subsetneq D_{G}\left(1, a_{n}\right) \text {. }
$$

$O$ inteiro $n \geq 0$ é denomina-se comprimento da cadeia acima.

b) Definimos o comprimento de cadeia de $G^{21}$ como o elemento de $\mathbb{N} \cup\{\infty\}$ definido por

$$
c l(G)=\sup \{m \geq 0 \text { : Existe uma cadeia de comprimento } m \text { em } G\} \text {. }
$$

\footnotetext{
${ }^{20} \mathrm{O}$ funtor descrito nos itens (a) e (b) da Proposição 3.47 .

21 "Chain length" em inglês.
} 
Dizemos que $G$ tem comprimento de cadeia finito se $c l(G)<\infty$. Indicamos por $\mathbf{R S G}_{c l f}$ a categoria dos grupos especiais reduzidos de comprimento de cadeia finito.

Definição 3.50. Seja $G$ um grupo especial e $H$ um subgrupo de $G$, com $-1 \in H$.

a) $H$ é uma seção de $G$ se existir $r: G \longrightarrow H$ tal que $r \circ \iota_{H}=I d_{H}$, onde $\iota_{H}: H \hookrightarrow G$ é a imersão natural de $H$ em $G$. Se $H$ é uma seção de $G$, seja

$$
X_{G, H}=\left\{G \stackrel{r}{\longrightarrow} H: r \text { é um } S G \text {-morfismo e } r \circ \iota_{H}=I d_{H}\right\}
$$

b) Definimos $S_{f i n}(G)=\{H: H$ é uma seção finita de $G\}$.

c) Seja $X_{G}^{+}=\bigcup\left\{X_{G, H}: H\right.$ é uma seção finita de $\left.G\right\}=\bigcup\left\{X_{G, H}: H \in S_{\text {fin }}(G)\right\}$.

Note que se $H$ é uma seção de $G$, então

* $H$ é um retrato de $G$ e portanto um subgrupo puro de $G$ (3.22.(b)); em particular, $H$ é um subgrupo especial de $G$;

* Se $r \in X_{G, H}$, então $r$ é um SG-morfismo regular e sobrejetor (3.33.(3)). Logo, 3.32 implica que $H$ e $G /$ ker $r$ são isomorfos.

* Se $G$ for reduzido então $H$ é reduzido e $\Delta_{r} \doteq k e r(r)$ é um subgrupo saturado de $G^{22}$ de indice finito.

Com estes preliminares enunciamos o seguinte resultado, que segue da Proposição 3:1.11 em [Ast1] :

Proposição 3.51. Seja $G$ um grupo especial reduzido de comprimento de cadeia finito. Seja

$$
G^{*}=\prod_{r \in X_{G}^{+}} G / \Delta_{r} .
$$

Então, a aplicação

$$
\nu: G \longrightarrow G^{*}, \text { dada por } \nu(g)=\left\langle g / \Delta_{r}\right\rangle_{r \in X_{G}^{+}}
$$

é um SG-morfismo puro.

Da Proposição 3.51 obtemos o

Corolário 3.52. Seja $G$ um grupo especial reduzido com comprimento de cadeia finito. Seja

$$
\mathcal{F}(G)=\{\Delta: \Delta \text { é um subgrupo saturado de indice finito em } G\} .
$$

Então, a aplicação

$$
\delta_{G}: G \longrightarrow \prod_{\Delta \in \mathcal{F}(G)} G / \Delta, \text { dada por } \delta_{G}(g)=\langle g / \Delta\rangle_{\Delta \in \mathcal{F}(G)}
$$

é um SG-morfismo puro.

\footnotetext{
${ }^{22} \operatorname{Ver} 3.27 .(\mathrm{f})$
} 
Prova. Como cada aplicação $p_{\Delta}: G \rightarrow G / \Delta: g \mapsto g / \Delta$ é um $S G$-morfismo (3.29.(a)), é imediato que $\delta_{G}$ é um SG-morfismo ${ }^{23}$.

Já observamos que para cada $r \in X_{G}^{+}$temos $\Delta_{r}=\operatorname{ker} r \in \mathcal{F}(G)$ e é fácil ver que a aplicação

$$
r \in X_{G}^{+} \stackrel{\Omega_{G}}{\mapsto} \Delta_{r} \in \mathcal{F}(G)
$$

é injetora.

Com a notação em 3.51 , seja

$$
q: \prod_{\Delta \in \mathcal{F}(G)} G / \Delta \longrightarrow G^{*}
$$

a projeção que esquece as coordenadas fora de imagem de $\Omega_{G}$. Assim temos o seguinte diagrama comutativo

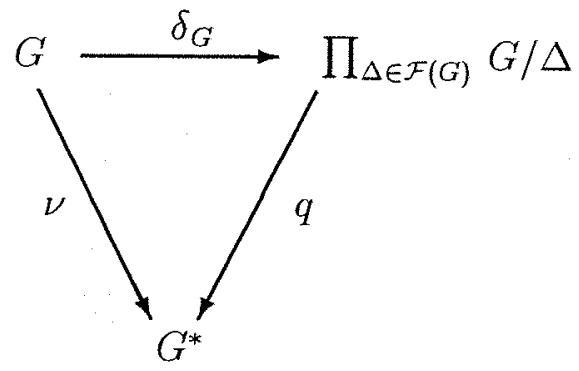

onde $\nu$ é um SG-morfismo puro. Segue do item (a).(2) da Proposição 2.45 que $\delta_{G}$ é puro, como desejado.

O próximo resultado sobre grupos de comprimento de cadeia finito pode ser extraído da Proposição 1.3 em [Ast2]. Uma vez que esta referência ainda não está amplamente disponível, apresentaremos uma prova do mesmo.

Proposição 3.53. Sejam $G$ um grupo especial reduzido, $k \geq 1$ um inteiro e $\varphi$ uma n-forma sobre $G$. Suponha que

$* G \vDash \operatorname{ind}_{W}(\varphi)<k$;

* Para todo subgrupo saturado próprio em $G, G / \Delta \models \operatorname{ind}_{W}(\varphi) \geq k$.

Então $G$ é um grupo especial de comprimento de cadeia finito.

Prova. Se $\operatorname{card}(G) \leqslant 2$ não há nada a ser demonstrado. Senão podemos considerar um inteiro $l>1$ e tomar uma cadeia de comprimento $l$ em $G$ :

$$
D_{G}\left(1, a_{0}\right) \subsetneq D_{G}\left(1, a_{1}\right) \subsetneq \ldots \subsetneq D_{G}\left(1, a_{l}\right)
$$

onde $a_{0}=1$ e $a_{l}=-1$.

Assim, por [SG2] em 3.1 temos $D_{G}\left(1, a_{l}\right)=G$. Como $G$ satisfaz [red] temos $D_{G}\left(1, a_{0}\right)=\{1\}$. Como $G$ é reduzido ainda temos que

\footnotetext{
${ }^{23}$ Obtido pela propriedade universal dos produtos.
} 


$$
\text { Para todo } 0 \leq i<l, \quad a_{i} a_{i+1} \neq 1,-1 .
$$

De fato, está claro que $a_{i}=a_{i+1}$ viola a condição de a sequência em (I) ser estritamente crescente. Por outro lado, se existisse $i<l$ tal que $a_{i} a_{i+1}=-1$, obteríamos, lembrando 3.8.(b) e o axioma [SG4] em 3.1,

$$
\begin{aligned}
a_{i} \in D_{G}\left(1, a_{i+1}\right) & \Rightarrow\left\langle a_{i}, a_{i} a_{i+1}\right\rangle \equiv_{G}\left\langle 1, a_{i+1}\right\rangle \quad \Rightarrow \quad\left\langle a_{i},-1\right\rangle \equiv_{G}\left\langle 1, a_{i+1}\right\rangle \\
& \Rightarrow\left\langle a_{i},-a_{i+1}\right\rangle \equiv_{G}\langle 1,1\rangle
\end{aligned}
$$

e o axioma da redução acarretaria que $a_{i}=1$ e $a_{i+1}=-1$; assim $a_{0}=a_{i}$ e $a_{i+1}=a_{l}$ e concluiríamos que $l=1$, contrariando nossa escolha em (I).

Segue imediatamente de (II) e de 3.27.(d) que

$$
\forall 1 \leq i \leq l, \quad\{1\} \neq D_{G}\left(1, a_{i} a_{i-1}\right) \text { é um subgrupo saturado próprio de } G \text {. }
$$

Fato 3.54. Sejam $G$ um grupo especial reduzido e $u \in G$. Sejam $\varphi, \psi$ formas de mesma dimensão sobre $G$ e $P$ uma forma de Pfister com coeficientes em $D_{G}(1, u)$. Então,

$$
P \otimes \varphi \equiv_{G} P \otimes \psi \quad \Rightarrow \quad\langle 1, u\rangle \otimes \varphi \equiv_{G}\langle 1, u\rangle \otimes \psi \text {. }
$$

Prova. Como $P$ tem coeficientes em $D_{G}(1, u)$, os itens (d) e (c) de 3.27 (nesta ordem) garantem que $D_{G}(P) \subseteq D_{G}(1, u)$. A conclusão desejada é então consequência de 3.27.(e).

Para $1 \leq i \leq l$, (III) e as hipóteses da Proposição implicam que, com $\Delta_{i}=D_{G}\left(1, a_{i} a_{i-1}\right)$,

$$
p_{\Delta_{i}} \star \varphi \text { tem índice de Witt } \geq k \text { em } G / \Delta_{i} \text {. }
$$

A equivalência em 3.29.(b) e o Fato 3.54 fornecem, para cada $1 \leq i \leq l$, uma forma $\psi_{i}$, de dimensão $n-2 k$ sobre $G$ tal que

$$
\begin{aligned}
\left\langle 1, a_{i} a_{i-1}\right\rangle \otimes \varphi & \equiv_{G} \quad\left\langle 1, a_{i} a_{i-1}\right\rangle \otimes\left(\psi_{i} \oplus k\langle 1,-1\rangle\right) \\
& \equiv_{G} \quad\left(\left\langle 1, a_{i} a_{i-1}\right\rangle \otimes \psi_{i}\right) \oplus \quad\left(\left\langle 1, a_{i} a_{i-1}\right\rangle \otimes k\langle 1,-1\rangle\right) .
\end{aligned}
$$

Note que se $u \in G$, então axioma [SG2] em 3.1 acarreta

$$
\begin{aligned}
\langle 1, u\rangle \otimes k\langle 1,-1\rangle & =k(\langle 1, u\rangle \otimes\langle 1,-1\rangle)=k\langle 1, u,-1,-u\rangle \equiv_{G} k(\langle 1,-1\rangle \oplus\langle u,-u\rangle) \\
& \equiv_{G} k(\langle 1,-1\rangle \oplus\langle 1,-1\rangle) \equiv_{G} 2 k\langle 1,-1\rangle,
\end{aligned}
$$

que substituída (com $u=a_{i} a_{i-1}$ ) em (IV) fornece, para cada $1 \leq i \leq l$,

$$
\left\langle 1, a_{i} a_{i-1}\right\rangle \otimes \varphi \equiv_{G}\left(\left\langle 1, a_{i} a_{i-1}\right\rangle \otimes \psi_{i}\right) \oplus 2 k\langle 1,-1\rangle .
$$

Somando ambos os membros das isometrias em (V), entre 1 e $l$, obtemos

$$
\oplus_{i=1}^{l}\left\langle 1, a_{i} a_{i-1}\right\rangle \otimes \varphi \equiv_{G} \bigoplus_{i=1}^{l}\left(\left\langle 1, a_{i} a_{i-1}\right\rangle \otimes \psi_{i}\right) \oplus(2 k\langle 1,-1\rangle)
$$

que transformações elementares levam a

$$
\varphi \otimes \bigoplus_{i=1}^{l}\left\langle 1, a_{i} a_{i-1}\right\rangle \equiv_{G} 2 k l\langle 1,-1\rangle \oplus \theta
$$

onde $\theta=\bigoplus_{i=1}^{l}\left\langle 1, a_{i} a_{i-1}\right\rangle \otimes \psi_{i}$. Agora observe que

$$
\bigoplus_{i=1}^{l}\left\langle 1, a_{i} a_{i-1}\right\rangle=l\langle 1\rangle \oplus\left\langle a_{1}, a_{1} a_{2}, a_{2} a_{3}, \ldots,-a_{l-1}\right\rangle
$$


Como $a_{1} \in D_{G}\left(1, a_{2}\right), 3.8$.(b) implica que $\left\langle a_{1}, a_{1} a_{2}\right\rangle \equiv_{G}\left\langle 1, a_{2}\right\rangle$. Esta isometria, substituída em (VII) fornece

$$
l\langle 1\rangle \oplus\left\langle a_{1}, a_{1} a_{2}, a_{2} a_{3}, \ldots,-a_{l-1}\right\rangle \equiv_{G} \quad l\langle 1\rangle \oplus\left\langle 1, a_{2}, a_{2} a_{3}, \ldots,-a_{l-1}\right\rangle .
$$

Novamente, temos $a_{2} \in D_{G}\left(1, a_{3}\right)$ e assim, $\left\langle a_{2}, a_{2} a_{3}\right\rangle \equiv_{G}\left\langle 1, a_{3}\right\rangle$, que nos leva a

$$
l\langle 1\rangle \oplus\left\langle a_{1}, a_{1} a_{2}, a_{2} a_{3}, \ldots,-a_{l-1}\right\rangle \equiv_{G} l\langle 1\rangle \oplus\left\langle 1,1, a_{3}, \ldots,-a_{l-1}\right\rangle .
$$

Prosseguindo com este método por $l-1$ passos e lembrando o axioma [SG2] em 3.1, obtemos

$$
\begin{array}{rl}
l\langle 1\rangle \oplus\left\langle 1 . a_{1}, a_{1} a_{2}, a_{2} a_{3}, \ldots,-1 . a_{l-1}\right\rangle & \equiv_{G} l\langle 1\rangle \oplus\left\langle 1,1, \ldots, a_{l-1},-a_{l-1}\right\rangle \\
\equiv_{G} & l\langle 1\rangle \oplus(l-2)\langle 1\rangle \oplus\langle 1,-1\rangle \\
\equiv_{G} & (2 l-2)\langle 1\rangle \oplus\langle 1,-1\rangle .
\end{array}
$$

Voltando com (VIII) e (VII) em (VI) fornece

$$
\varphi \otimes((2 l-2)\langle 1\rangle \oplus\langle 1,-1\rangle) \equiv_{G} \quad \theta \oplus 2 k l\langle 1,-1\rangle .
$$

Como $\varphi \otimes\langle 1,-1\rangle=\varphi-\varphi \equiv_{G} n\langle 1,-1\rangle$, (IX) pode reescrito como

$$
(2 l-2) \varphi \oplus n\langle 1,-1\rangle \equiv_{G} \quad \theta \oplus 2 k l\langle 1,-1\rangle .
$$

Seja $m=\operatorname{ind}_{W}(\varphi)$ em $G$; então existe uma forma anisotrópica $\psi$ sobre $G$ tal que

$$
\varphi \equiv_{G} \quad \psi \oplus m\langle 1,-1\rangle .
$$

subsituindo-se esta isometria em (X) obtemos

$$
(2 l-2) \psi \oplus((2 l-2) m+n)\langle 1 ;-1\rangle \equiv_{G} \quad \theta \oplus 2 k l\langle 1,-1\rangle .
$$

Uma vez que $\psi$ é anisotrópica e $G$ é reduzido, segue de 3.9.(e) que $(2 l-2) \psi$ também é uma forma anisotrópica. Assim, (XI) acarreta

$$
2 k l \leq(2 l-2) m+n
$$

Como $m<k$ (por hipótese) e $2 m \leq n^{24}$ temos $\frac{n-2 m}{2(k-m)} \geq 0$ e (XII) implica $l \leq \frac{n-2 m}{2(k-m)}$, mostrando que qualquer cadeia em $G$ é limitada e encerrando a prova.

\subsection{O Anel de Witt de um Grupo Especial}

Definição 3.55. Seja $G$ um grupo especial e $\varphi, \psi$ formas sobre $G$. Dizemos que $\varphi$ é Witt equivalente $a \psi$, em simbolos $\varphi \approx_{G} \psi$, se existirem naturais $n, m \geq 0$ tais que

$$
\varphi \oplus n\langle 1,-1\rangle \equiv_{G} \psi \oplus m\langle 1-1\rangle .
$$

Pelo cancelamento de Witt (3.9.(b)), esta relação pode sempre ser escrita como

$$
\varphi \oplus k\langle 1,-1\rangle \equiv \psi \quad \text { ou } \quad \varphi \equiv \psi \oplus k\langle 1,-1\rangle,
$$

onde $k=|\operatorname{dim} \varphi-\operatorname{dim} \psi| / 2$.

\footnotetext{
${ }^{24}$ Segue imediatamente da definição em 3.6.(e) que $2 \operatorname{ind}_{W}(\varphi) \leq \operatorname{dim} \varphi$; assim, $n-2 m \geq 0$.
} 
É fácil de verificar que $\approx_{G}$ é uma relação de equivalência no conjunto das formas sobre $G$. Para formas $\varphi, \psi$, sobre $G$, escrevemos

$$
\varphi-\psi \text { para indicar } \varphi \oplus-\psi \text {. }
$$

Seja $\mathcal{W}(G)$ o conjunto das classes de equivalência das formas sobre $G$ em relação a equivalência de Witt. Indicamos por $\bar{\varphi}$ a classe da forma $\varphi$ em $\mathcal{W}(G)$. Temos :

Lema 3.56. Seja $G$ um grupo especial e $\varphi$ uma forma sobre $G$.

a) $\varphi \otimes\langle 1,-1\rangle \equiv \operatorname{dim} \varphi \cdot\langle 1,-1\rangle=\sum_{i=1}^{\operatorname{dim} \varphi}\langle 1,-1\rangle$.

b) A equivalência de Witt é uma congruência em relação à soma e ao produto de formas.

c) Com as operaçôes $\bar{\varphi}+\bar{\psi}=\overline{\varphi \oplus \psi} \quad$ e $\bar{\varphi} \bar{\psi}=\overline{\varphi \otimes \psi}, \quad \mathcal{W}(G)$ é um anel comutativo com identidade $\overline{\langle 1\rangle}$, cujo neutro aditivo é a classe das formas hiperbólicas, denominado anel de Witt de $G$.

d) O conjunto $I(G)$ das (classes das) formas de dimensão par é um ideal maximal em $\mathcal{W}(G)$, o ideal fundamental de $\boldsymbol{G}$. Além disso, $\mathcal{W}(G) / I(G)$ é o corpo com dois elementos.

Se $G$ é um grupo especial, o anel graduado de Witt de $G$ é

$$
\mathcal{W}_{g}(G)=\left\langle\mathcal{W}(G) / I(G), I(G) / I^{2}(G), \ldots, I^{n}(G) / I^{n+1}(G), \ldots\right\rangle
$$

onde $I^{n}(G)$ é a $n^{\text {ésima }}$ potência do ideal fundamental $I(G)$ (3.56.(d)). Note que, como grupo abeliano, $I^{n}(G)$ é gerado pelas formas de Pfister de grau $n$ sobre $G$, isto é,

$$
\varphi \in I^{n}(G) \Leftrightarrow\left\{\begin{array}{l}
\text { Existem } m \geq 0, \epsilon_{1}, \ldots, \epsilon_{m} \in\{1,-1\} \text { e } P_{1}, \ldots, P_{m}, \text { formas } \\
\text { de } \\
\text { Pfister de grau } n \text { sobre } G \text { tal que } \varphi \approx_{G} \bigoplus_{i=1}^{m} \epsilon_{i} P_{i} .
\end{array}\right.
$$

A multiplicação graduada em $\mathcal{W}_{g}(G)$ é a induzida pelo produto tensorial de formas : para $\varphi \in I^{n}, \psi \in I^{m}$, se $\varphi / n$ indica $\varphi / I^{n+1}$, então

$$
\varphi / n * \psi / m=(\varphi \otimes \psi) / n+m
$$

3.57. A Conjectura de Marshall. Sejam $G$ um grupo especial reduzido e $\varphi$ uma forma sobre $G$. Com a notação em 3.18 , consideremos a condição

$[\mathrm{MC}(\varphi)] \quad \forall \sigma \in X_{G}, \sigma(\varphi) \equiv 0 \bmod 2^{n} \Rightarrow \varphi \in I^{n}(G)$.

Note que a hipótese em $[\mathrm{MC}(\varphi)]$ significa que a assinatura de $\varphi$ com respeito a todos os carácteres de $G$ é um inteiro congruente a 0 módulo $2^{n}$.

Dizemos que $G$ verifica a conjectura de Marshall, e escrevemos $G \models[\mathrm{MC}]$, se para toda forma $\varphi$ sobre $G$ vale $[\mathrm{MC}(\varphi)]$.

Em [DM2] foi provado que se $F$ é um corpo Pitagórico então $\mathcal{G}(F)$ verifica a conjectura de Marshall. Um problema aberto importante, proposto por M. Marshall na linguagem dos espaços de ordem abstratos em 1974, é se todo grupo especial reduzido satisfaz [MC]. 
Fixada a forma $\varphi \in I^{n}(G), \operatorname{com} \operatorname{dim} \varphi=k$, segue de $\left(^{*}\right)$ acima que existe uma fórmula existencial positiva em $L_{S G}, \Phi\left(v_{1}, \ldots, v_{k}\right)$, tal que

$\left(^{* *}\right) \quad G \models \Phi[\varphi] \quad \Leftrightarrow \quad \begin{aligned} & \text { Existem } a_{1}, \ldots, a_{m} \in G \text { e } P_{1}, \ldots, P_{m}, \text { formas de } \\ & \text { Pfister de grau } n \text { sobre } G \text {, tais que } \varphi \approx_{G} \bigoplus_{i=1}^{m} a_{i} P_{i} .\end{aligned}$

Esta observação nos fornece

Lema 3.58. Seja $f: G \longrightarrow H$ um morfismo puro de grupos especiais reduzidos. Então,

$$
H \vDash[\mathrm{MC}] \quad \Rightarrow \quad G \models[\mathrm{MC}] \text {. }
$$

Prova. Seja $\varphi$ uma forma sobre $G$ de dimensão $k$ tal que para todo $\sigma \in X_{G}, \sigma(\varphi) \equiv 0 \bmod 2^{n}$. Seja $\tau \in X_{H}$; então $\tau \circ f \in X_{G}$ e portanto

$$
\tau(f \star \varphi)=(\tau \circ f)(\varphi) \equiv 0 \bmod 2^{n} .
$$

Como $H \models[M C], f \star \varphi \in I^{n}(H)$; como observado acima em $\left(^{* *}\right)$, existe uma fórmula existencial positiva em $L_{S G}, \Phi\left(v_{1}, \ldots, v_{k}\right)$, tal que $H \models \Phi[f \star \varphi]$. Como $f$ é um SG-morfismo puro, concluímos que $G \models \Phi[\varphi]$ e portanto, novamente por $\left(^{* *}\right), \varphi \in I^{n}(G)$, como desejado. 


\section{Capítulo 4}

\section{A Adjunção Induzida por um SG-Morfismo}

Neste Capítulo apresentamos, num primeiro momento, as noções de adjunção induzida e morfismos derivados de um morfismo entre grupos especiais reduzidos; em seguida, analisamos alguns subconjuntos do reticulado dos subgrupos saturados de um grupo especial reduzido e o comportamento destes pelas adjunções induzidas por morfismos.

Neste Capítulo, todos os grupos especiais são reduzidos.

\subsection{A Adjunção Induzida}

É bem conhecido que cada função entre conjuntos origina um par adjunto de funções crescentes entre seus conjuntos de partes correspondentes ${ }^{1}$. Analogamente, iremos associar a cada $\mathrm{SG}$-morfismo entre grupos especiais reduzidos um par adjunto de funções crescentes entre seus reticulados completos de subgrupos saturados.

Nesta seção examinaremos este fato e suas consequências, sendo que a principal para nosso trabalho - a definição da envoltória profinita de um RSG (seção 5.5) - , é a obtenção dos morfismos derivados de um $R S G$-morfismo. fornece

Lembramos que se $A \subseteq G$ é um subgrupo de um grupo especial $G$, o item (b) do Lema 3.27

$$
A^{\text {sat }}=\bigcup\left\{D_{G}(\psi): \psi \text { é uma forma de Pfister sobre } A\right\}
$$

é o menor subgrupo saturado de $G$ que contém $A$. Além disso, por 3.27.(f), imagem inversa por um SG-morfismo preserva saturação. Com este preliminares introduzimos a

Definição 4.1. (A adjunção induzida) Seja $f: G \longrightarrow G^{\prime}$ um morfismo em RSG. Definimos

$$
f_{*}: \operatorname{Ssat}(G) \longrightarrow \operatorname{Ssat}\left(G^{\prime}\right) \quad \text { e } \quad f^{*}: \operatorname{Ssat}\left(G^{\prime}\right) \longrightarrow \operatorname{Ssat}(G)
$$

dadas, para $\Delta \in S$ sat $(G)$ e $\Delta^{\prime} \in S$ sat $\left(G^{\prime}\right)$, por

\footnotetext{
${ }^{1}$ As funções "imagem direta" e "imagem inversa" induzidas pela função dada.
} 


$$
f_{*}(\Delta)=(f[\Delta])^{\text {sat }} \text { e } f^{*}\left(\Delta^{\prime}\right)=f^{-1}\left[\Delta^{\prime}\right]
$$

$f_{*} e^{\prime} a$ imagem saturada de $f e f^{*} a$ imagem inversa saturada de $f$.

Proposição 4.2. Sejam $G \stackrel{f}{\longrightarrow} G^{\prime} \stackrel{g}{\longrightarrow} G^{\prime \prime}$ morfismos em RSG.

a) $f_{*} e f^{*}$ são crescentes, formando um par adjunto ${ }^{2}$, isto é,

$$
\text { Para } \Delta \in \operatorname{Ssat}(G) \text { e } \Delta^{\prime} \in \operatorname{Ssat}\left(G^{\prime}\right), f_{*}(\Delta) \subseteq \Delta^{\prime} \Leftrightarrow \Delta \subseteq f^{*}\left(\Delta^{\prime}\right) .
$$

Em particular, $\Delta \subseteq f^{*}\left(f_{*}(\Delta)\right)$ e $f_{*}\left(f^{*}\left(\Delta^{\prime}\right)\right) \subseteq \Delta^{\prime}$.

b) $(1)(g \circ f)_{*}=g_{*} \circ f_{*}$;

(2) $(g \circ f)^{*}=f^{*} \circ g^{*}$;

(3) $I d_{G}^{*}=I d_{S s a t(G)}=\left(I d_{G}\right)_{*}$.

c) As seguintes condições são equivalentes : ${ }^{3}$

(1) $f$ é uma imersão completa;

(2) Para todo $\Delta \in S \operatorname{sat}(G), \Delta=f^{*}\left(f_{*}(\Delta)\right)$.

d) Se $f$ é sobrejetor e $\Delta^{\prime} \in S$ sat $\left(G^{\prime}\right)$, então $f_{*}\left(f^{*}\left(\Delta^{\prime}\right)\right)=\Delta^{\prime}$.

e) Se $f$ é sobrejetor e regular (3.31) então para todo $\Delta \in S$ Sat $(G)$

$$
\text { ker } f \subseteq \Delta \Rightarrow f_{*}(\Delta)=f[\Delta] \text { e } f^{*}\left(f_{*}(\Delta)\right)=\Delta \text {. }
$$

Prova. O item (a) segue imediatamente das definições e das propriedades usuais da imagem e da imagem inversa. Notamos ainda que, por 3.27.(b), segue que

$$
f_{*}(\Delta)=\bigcup\left\{D_{G^{\prime}}(f \star \psi): \psi \text { é uma forma de Pfister sobre } \Delta\right\}^{4} .
$$

b) Os itens (2) e (3) em (b) estão claros. Para (1), seja $\Delta \in S$ sat $(G)$; no sentido de facilitar a leitura, indicaremos $A^{\text {sat }}$ por $\bar{A}(A \subseteq G)$. Nesta notação, devemos verificar que

$$
\overline{g[f[\Delta]]}=\overline{g[\overline{f[\Delta]}]} \text {. }
$$

Como a imagem por uma função é crescente e $f[\Delta] \subseteq \overline{f[\Delta]}$, é suficiente provar que

$$
\overline{g[\overline{f[\Delta]}]} \subseteq \overline{g[f[\Delta]]}
$$

Seja $x \in \overline{g[\overline{f[\Delta]}]}$; por 3.27.(b) existe uma forma de Pfister $\psi$ com coeficientes em $g[\overline{f[\Delta]}]$ tal que $x \in D_{G^{\prime \prime}}(\psi)$. Podemos escrever

$$
\psi=\bigotimes_{i=1}^{n}\left\langle 1, g\left(a_{i}\right)\right\rangle
$$

onde $a_{i} \in \overline{f[\Delta]}, 1 \leq i \leq n$. Uma nova aplicação de 3.27 .(b) fornece, para cada $1 \leq i \leq n$, uma forma de Pfister $P_{i}$, com coeficientes em $\Delta$, tal que

$$
\text { Para todo } 1 \leq i \leq n, \quad a_{i} \in D_{G^{\prime}}\left(f \star P_{i}\right) .
$$

\footnotetext{
${ }^{2}$ Alguns autores usam a terminologia conexão de Galois covariante (ver a Observação 1.34).

${ }^{3}$ Outras caracterizações de monomorfismos completos aparecem no Teorema 5.2 em [DM1].

${ }^{4}$ Note que se $f$ é um SG-morfismo e $Q=\bigotimes_{i=1}^{n}\left\langle 1, d_{i}\right\rangle$, então $f \star Q=\bigotimes_{i=1}^{n}\left\langle 1, f\left(d_{i}\right)\right\rangle$.
} 
Seja $P=\bigotimes_{i=1}^{n} P_{i}$; então $P$ é uma forma de Pfister sobre $\Delta$ e por 3.9.(g), temos

$$
\bigcup_{i=1}^{n} D_{G^{\prime}}\left(f \star P_{i}\right) \subseteq D_{G^{\prime}}(f \star P)
$$

Consequentemente, $\left\{a_{1}, \ldots, a_{n}\right\} \subseteq D_{G^{\prime}}(f \star P)$ e portanto

$$
\left\{g\left(a_{1}\right), \ldots, g\left(a_{n}\right)\right\} \subseteq D_{G^{\prime \prime}}((g \circ f) \star P) .
$$

Agora, os itens (c) e (d) em 3.27.(d) fornecem

mostrando que $x \in \overline{g[f[\Delta]]}$, como necessário. ${ }^{5}$

$$
x \in D_{G^{\prime \prime}}(\psi) \subseteq D_{G^{\prime \prime}}((g \circ f) \star P)
$$

c) Começamos mencionando o seguinte Fato, parte do enunciado do Teorema 5.2 em [DM1] :

Fato 4.3. Se $h: G \longrightarrow H$ é um morfismo de grupos especiais reduzidos, as seguintes condições são equivalentes:

(i) h é uma imersão completa;

(ii) $h$ é injetora e para toda forma de Pfister $\varphi$ sobre $G, D_{H}(h \star \varphi) \cap \operatorname{Im}(h)=h\left[D_{G}(\varphi)\right]$.

$(1) \Rightarrow(2)$ : Uma vez que $f[\Delta] \subseteq f_{*}(\Delta)$ e $f^{*}$ é a imagem inversa por $f$, é suficiente mostrar que

$$
f^{*}\left(f_{*}(\Delta)\right)=f^{*}(\overline{f[\Delta]}) \subseteq \Delta .
$$

Seja $x \in G$ tal que $f(x) \in \overline{f[\Delta]}$; por 3.27.(b), existe uma forma de Pfister $P$ com coeficientes em $\Delta$ tal que

$$
f(x) \in D_{G^{\prime}}(f \star P) .
$$

Pela equivalência no Fato 4.3, concluímos que $f(x) \in f\left[D_{G}(P)\right]$ e o fato que $f$ é injetora acarreta $x \in D_{G}(P)$. Como $P$ tem coeficientes em $\Delta, 3.27$.(c) implica $x \in \Delta$, como desejado.

$(2) \Rightarrow(1)$ : Para vermos que $f$ é injetor, lembrando a Observação 3.28.(a), temos que

$$
\left\{1_{G}\right\}=f^{*}\left(f_{*}\left(\left\{1_{G}\right\}\right)\right)=f^{*}\left(\overline{\left\{1_{G^{\prime}}\right\}}\right)=f^{-1}\left[\left\{1_{G^{\prime}}\right\}\right]=\operatorname{ker} f
$$

como necessário. Seja $P$ uma forma de Pfister sobre $G$. Como $f$ é um SG-morfismo, vemos pelo Fato 4.3 que é suficiente verificar que

$$
D_{G^{\prime}}(f \star P) \cap \operatorname{Im} f \subseteq f\left[D_{G}(P)\right] .
$$

Por 3.27.(d), $\Delta=D_{G}(P)$ é um subgrupo saturado de $G$. Observe que os coeficientes da forma de Pfister $f \star P\left(\right.$ sobre $G^{\prime}$ ) estão em $f[\Delta]$; assim

$$
D_{G^{\prime}}(f \star P) \subseteq \overline{f[\Delta]} \text {. }
$$

Por outro lado, (2) acarreta

$$
\Delta=f^{*}\left(f_{*}(\Delta)\right)=f^{*}(\overline{f[\Delta]})
$$

que juntamente com $(+)$ implica

\footnotetext{
${ }^{5}$ Está claro que $(g \circ f) \star P$ é uma forma de Pfister sobre $g[f[\Delta]]$.
} 


$$
D_{G^{\prime}}(f \star P) \cap \operatorname{Im} f \subseteq \overline{f[\Delta]} \cap \operatorname{Im} f=f[\Delta],
$$

encerrando a prova de (c). O item (d) está claro.

e) Seja ker $f \subseteq \Delta$ um subgrupo saturado de $G$. Para mostrar que $f_{*}(\Delta)=\Delta$ é suficiente verificar que $f[\Delta]$ é saturado. Mas isto segue imediatamente da Proposição 3.32 e do Fato 3.34. A última afirmação é agora imediata, encerrando a prova.

\subsection{Morfismos Derivados}

4.4. Construção. Seja $f: G \longrightarrow G^{\prime}$ um morfismo de grupos especiais reduzidos. Sejam $\Sigma \in \operatorname{Ssat}(G)$ e $\Delta^{\prime} \in \operatorname{Ssat}\left(G^{\prime}\right)$, tais que $\underline{\Sigma \subseteq f^{*}\left(\Delta^{\prime}\right)}{ }^{6}$. Consideremos a composição

$$
G \stackrel{f}{\longrightarrow} G^{\prime} \stackrel{p_{\Delta^{\prime}}}{\longrightarrow} G^{\prime} / \Delta^{\prime} .
$$

Note que o núcleo desta composição é exatamente $\Delta=f^{*}\left(\Delta^{\prime}\right)$. Pelo Teorema 3.29.(c), existem e são únicos, SG-morfismos

$$
\left\{\begin{array}{cc}
f_{\Sigma, \Delta^{\prime}} & : G / \Sigma \longrightarrow G^{\prime} / \Delta^{\prime} \\
f_{\Delta^{\prime}} & : G / \Delta \longrightarrow G^{\prime} / \Delta^{\prime}
\end{array}\right.
$$

que fazem os seguintes diagramas comutar :
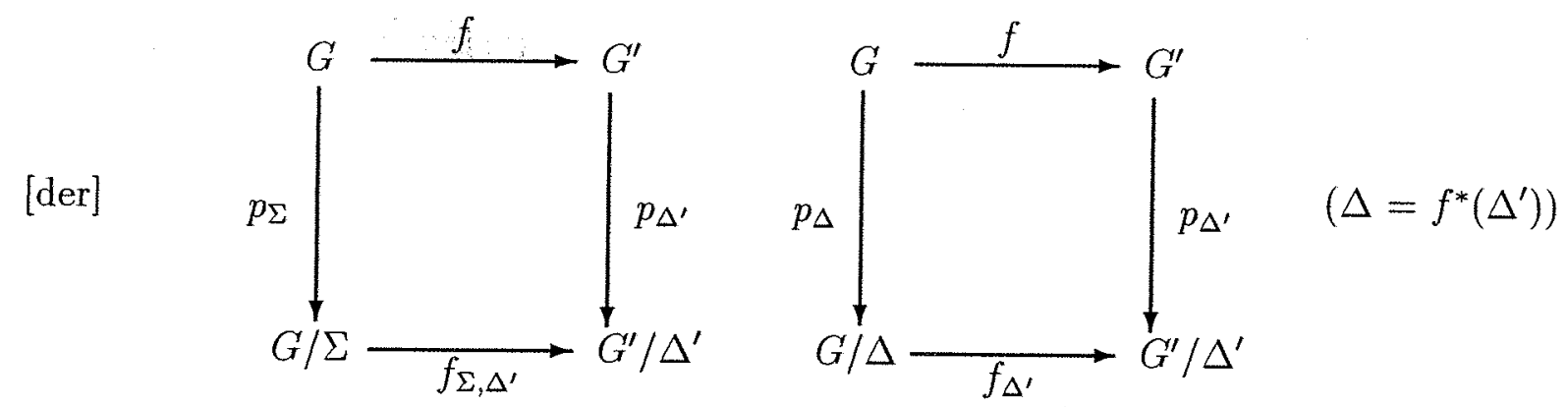

onde $p_{*}$ são as SG-projeções canônicas. Note que $f_{\Delta^{\prime}}$ é injetor.

Definição 4.5. (Morfismos derivados) Seja $f: G \longrightarrow G^{\prime}$ um morfismo em RSG. Para $\Delta^{\prime} \in \operatorname{Ssat}\left(G^{\prime}\right)$ e $\Sigma \in S$ sat $(G)$, com $\Sigma \subseteq f^{*}\left(\Delta^{\prime}\right)$, os $S G$-morfismos $f_{\Delta^{\prime}}$ e $f_{\Sigma, \Delta^{\prime}}$ construídos acima denominam-se morfismos derivados de $f$.

Algumas das propriedades básicas dos morfismos derivados constam da

Proposição 4.6. Sejam $G \stackrel{f}{\longrightarrow} G^{\prime} \stackrel{g}{\longrightarrow} G^{\prime \prime}$ morfismos de grupos especiais reduzidos.

\footnotetext{
${ }^{6}$ Equivalentemente $f_{*}(\Sigma) \subseteq \Delta^{\prime}$, por 4.2.(a).
} 
a) Para $\Delta \in \operatorname{Ssat}(G),\left(I d_{G}\right)_{\Delta}=I d_{G / \Delta}$.

b) Para $\Delta^{\prime \prime} \in S$ sat $\left(G^{\prime \prime}\right)$, sejam $\Delta^{\prime}=g^{*}\left(\Delta^{\prime \prime}\right)$ e $\Delta=f^{*}\left(g^{*}\left(\Delta^{\prime \prime}\right)\right)$. Então,

$$
(g \circ f)_{\Delta^{\prime \prime}}=g_{\Delta^{\prime \prime}} \circ f_{\Delta^{\prime}} \text {. }
$$

c) Sejam $\Delta^{\prime} \subseteq \Sigma^{\prime}$ em Ssat $\left(G^{\prime}\right), \Delta=f^{*}\left(\Delta^{\prime}\right)$ e $\Sigma=f^{*}\left(\Sigma^{\prime}\right)$. Sejam $p_{\Delta \Sigma}, p_{\Delta^{\prime} \Sigma^{\prime}}$ únicos $S G$-morfismos que fazem os dois primeiros diagramas abaixo comutarem ${ }^{7}$ :
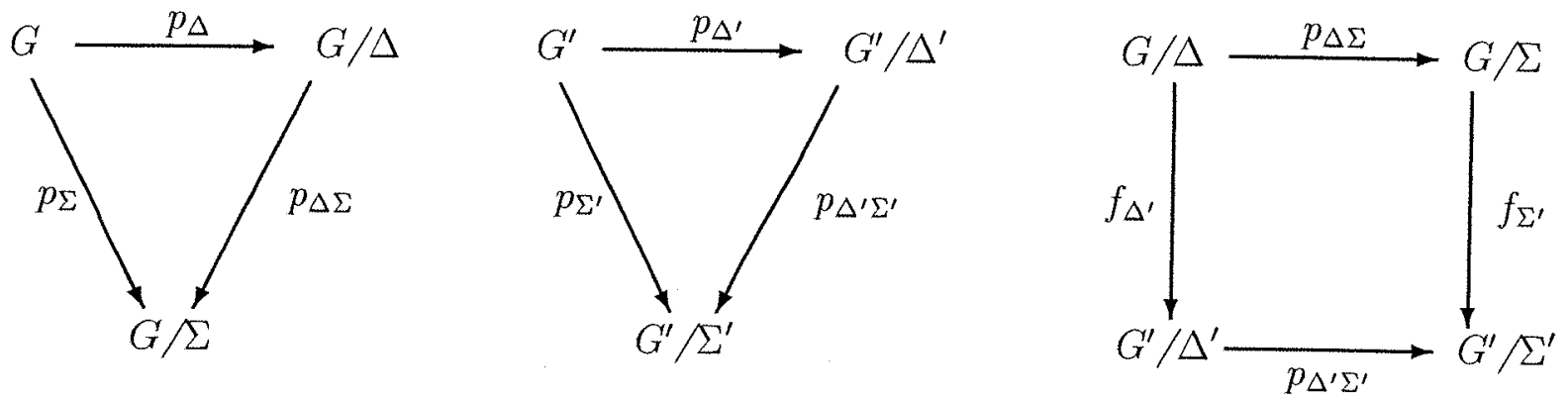

Entẫo, $f_{\Sigma^{\prime}} \circ p_{\Delta \Sigma}=p_{\Delta^{\prime} \Sigma^{\prime}} \circ f_{\Delta^{\prime}}$, isto é o quadrado acima é comutativo.

d) Se $f$ é uma imersão completa, $\Delta \in S$ sat $(G)$ e $\Delta^{\prime}=f_{*}(\Delta)$, então $\Delta=f^{*}\left(\Delta^{\prime}\right)$ e também temos que $f_{\Delta^{\prime}}: G / \Delta \longrightarrow G^{\prime} / \Delta^{\prime}$ é uma imersão completa.

e) Se $f$ é sobrejetor, $\Delta^{\prime} \in S \operatorname{sat}\left(G^{\prime}\right)$ e $\Delta=f^{*}\left(\Delta^{\prime}\right)$ então $f_{\Delta^{\prime}}: G / \Delta \longrightarrow G^{\prime} / \Delta^{\prime}$ é um SG-morfismo bijetor.

f) Suponha que $f$ é sobrejetora e regular ${ }^{8}$. Se $\Delta^{\prime} \in S$ sat $\left(G^{\prime}\right)$ e $\Delta=f^{*}\left(\Delta^{\prime}\right)$ então $f_{\Delta^{\prime}}$ é um $S G$-isomorfismo entre $G / \Delta$ e $G^{\prime} / \Delta^{\prime}$.

Prova. O item (a) está claro. Para (b), seja $\Delta^{\prime \prime} \in S$ sat $\left(G^{\prime \prime}\right)$; com a notação do enunciado, temos por 4.2.(b) que $\Delta=(g \circ f)^{*}\left(\Delta^{\prime \prime}\right)$ e segue diretamente das definições que o diagrama abaixo é comutativo:

\footnotetext{
${ }^{7}$ Outra aplicação do Teorema 3.29 .

${ }^{8}$ Definida em 4.2.(e).
} 


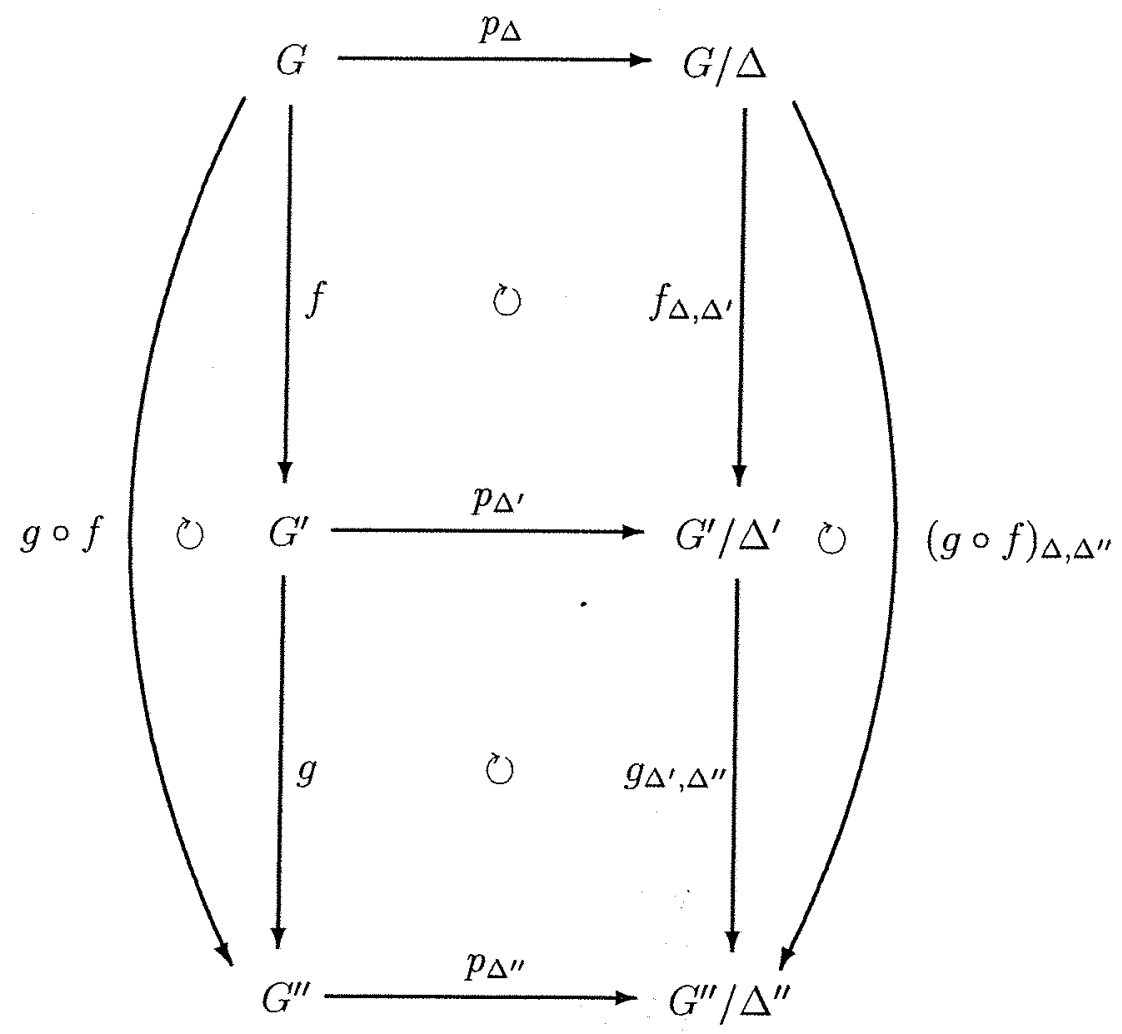

c) Seja $\Delta^{\prime} \subseteq \Sigma^{\prime}$ em $S s a t\left(G^{\prime}\right)$; com a notação do enunciado, note que como $p_{\Delta}$ é sobrejetor, temos

$$
f_{\Sigma^{\prime}} \circ p_{\Delta \Sigma}=p_{\Delta^{\prime} \Sigma^{\prime}} \circ f_{\Delta^{\prime}} \Leftrightarrow f_{\Sigma^{\prime}} \circ p_{\Delta \Sigma} \circ p_{\Delta}=p_{\Delta^{\prime} \Sigma^{\prime}} \circ f_{\Delta^{\prime}} \circ p_{\Delta} .
$$

Tendo em conta o diagrama da direita em [der] (4.4, página 116) juntamente com os diagramas no enunciado, obtemos

$$
f_{\Sigma^{\prime}} \circ p_{\Delta \Sigma} \circ p_{\Delta}=f_{\Sigma^{\prime}} \circ p_{\Sigma}=p_{\Sigma^{\prime}} \circ f=p_{\Delta^{\prime} \Sigma^{\prime}} \circ p_{\Delta^{\prime}} \circ f=p_{\Delta^{\prime} \Sigma^{\prime}} \circ f_{\Delta^{\prime}} \circ p_{\Delta},
$$

como necessário, isto é o diagrama abaixo comuta :

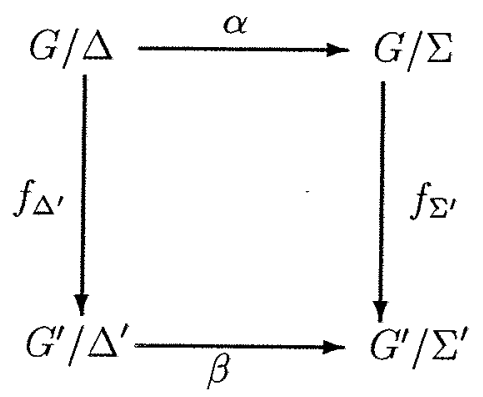

onde $\alpha=p_{\Delta \Sigma} \quad$ e $\beta=p_{\Delta^{\prime} \Sigma^{\prime}}$

d) Seja $\Delta \in S$ sat $(G)$; pelo item (d) em 4.2 sabemos que $\Delta=f^{*}\left(f_{*}(\Delta)\right)$. Assim, temos um $\mathrm{SG}$-morfismo injetor (veja comentário em negrito no final de 4.4)

$$
f_{\Delta^{\prime}}: G / \Delta \longrightarrow G^{\prime} / \Delta^{\prime}
$$


onde $\Delta^{\prime}=f_{*}(\Delta)$. Sejam $\varphi, \psi$ formas sobre $G / \Delta$ de mesma dimensão. Para mostrar que $f_{\Delta^{\prime}}$ é completo é suficiente verificar que

$$
f_{\Delta^{\prime}} \star \varphi \equiv_{G^{\prime} / \Delta^{\prime}} f_{\Delta^{\prime}} \star \psi \Rightarrow \varphi \equiv_{G / \Delta} \psi \text {. }
$$

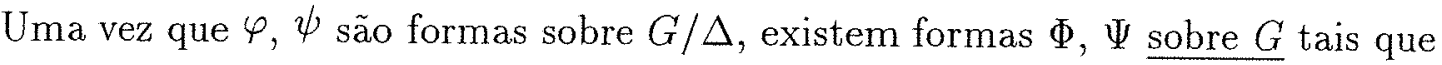

$$
\varphi=p_{\Delta} \star \Phi \text { e } \psi=p_{\Delta} \star \Psi,
$$

onde $p_{\Delta}: G \longrightarrow G / \Delta$ é a SG-projeção canônica. Lembrando que o diagrama da direita em [der] (4.4, página 116), isto é,

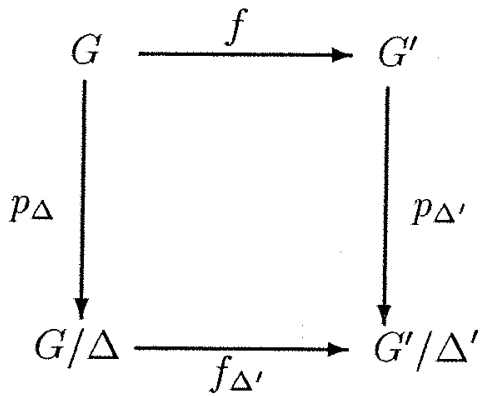

é comutativo, a hipótese em (I) juntamente com (II) implicam

$$
\left(p_{\Delta^{\prime}} \circ f\right) \star \Phi \equiv_{G^{\prime} / \Delta^{\prime}}\left(p_{\Delta^{\prime}} \circ f\right) \star \Psi .
$$

Pelo Teorema 3.29.(b), existe uma forma de Pfister $Q$ com coeficientes em $\Delta^{\prime}=f_{*}(\Delta)=f[\Delta]^{\text {sat }}$ tal que

$$
Q \otimes(f \star \Phi) \equiv_{G^{\prime}} \quad Q \otimes(f \star \Psi) .
$$

Com o mesmo método utilizado na prova de 4.2.(b).(1) ${ }^{9}$, podemos encontrar uma forma de Pfister, $R$, com coeficientes em $\Delta$, tal que $D_{G^{\prime}}(Q) \subseteq D_{G^{\prime}}(f \star R)$. Esta relação, 3.27.(e), (III) e 3.16 fornecem

$$
f \star(R \otimes \Phi)=(f \star R) \otimes(f \star \Phi) \equiv_{G^{\prime}}(f \star R) \otimes(f \star \Psi)=f \star(R \otimes \Psi) .
$$

Uma vez que $f$ é uma imersão completa, a relação acima implica que

$$
R \otimes \Phi \equiv_{G} \quad R \otimes \Psi .
$$

Outra aplicação de 3.29.(b) implica, já que $R$ tem coeficientes em $\Delta$, acarreta

$$
\varphi=p_{\Delta} \star \Phi \equiv_{G / \Delta} \quad p_{\Delta} \star \Psi=\psi
$$

estabelecendo (I), conforme desejado.

Os itens (e) e (f) seguem facilmente das definições e omitiremos sua verificação.

Observação 4.7. Na notação vigente no item (d) da proposição acima vimos que se $f$ é $S G$ imersão completa então $f_{\Delta^{\prime}}$ também é $S G$-imersão completa. De fato, tomando $f$ imersão completa (respectivamente: $S G$-morfismo que reflete isotropia, $S G$-morfismo que reflete subformas, $L_{S G}$-morfismo puro) podemos concluir que $f_{\Delta}$ 'é imersão completa (respectivamente:

\footnotetext{
${ }^{9} \mathrm{Se} Q=\otimes\left\langle 1, a_{i}\right\rangle$, para cada $a_{i}$ existe $R_{i} \in \operatorname{Pfister}(\Delta)$ tal que $a_{i} \in D_{G^{\prime}}\left(f \star R_{i}\right)$; tomamos $R=\bigotimes R_{i}$.
} 
$S G$-morfismo que reflete isotropia, $S G$-morfismo que reflete subformas, $L_{S G}$-morfismo puro): basta adaptarmos a prova realizada, utilizando as proposições 3.36 e 3.37 .

\subsection{Sobre alguns subconjuntos de subgrupos saturados}

Nesta seção consideramos certos subconjuntos do reticulado dos subgrupos saturados de um grupo especial e analisamos o comportamento desses subconjuntos pelas flechas das adjunções induzidas por morfismos entre grupos especiais.

Definição 4.8. Seja $G$ um grupo especial reduzido. Definimos :

a) $S$ sat ${ }^{\star}(G)=\{\Delta \in S$ sat $(G): \operatorname{card}(G / \Delta)>1\}$, o conjunto dos subgrupos saturados próprios de $G$.

b) $\mathcal{F}(G)=\{\Delta \in S \operatorname{sat}(G): \operatorname{card}(G / \Delta)<\omega\}$, o conjunto dos subgrupos saturados de indice finito em $G$.

c) $X(G)=\{\Sigma \in S$ sat $(G): \operatorname{card}(G / \Sigma):=2\}$ é o conjunto dos subgrupos saturados maximais de $G$.

Temos também a seguinte versão relativa dos conjuntos acima :

Definição 4.9. Se $G$ é um grupo especial reduzido e $\Theta \in S$ sat $(G)$, definimos

a) $\operatorname{Ssat}_{\Theta}(G)=\{\Delta \in S \operatorname{sat}(G): \Theta \subseteq \Delta\}$.

b) $\operatorname{Ssat}_{\Theta}^{\star}(G)=\operatorname{Ssat}^{\star}(G) \cap \operatorname{Ssat}_{\Theta}(G)$.

c) $\mathcal{F}_{\Theta}(G)=\mathcal{F}(G) \cap \operatorname{Ssat}_{\Theta}(G)$.

d) $X_{\Theta}(G)=X(G) \cap \operatorname{Ssat}_{\Theta}(G)$.

O Lema abaixo justifica a nomenclatura adotada acima:

Lema 4.10. Seja $G$ um grupo especial reduzido e $\Delta \in S$ sat $(G)$.

a) As seguintes condições são equivalentes :
(1) $\Delta \in S \operatorname{sat}^{\star}(G)$;
(2) $-1 \notin \Delta$;
(3) $\Delta \subsetneq G$

b) As seguintes condições são equivalentes:
(1) $\Delta \in X(G)$;
(2) $G / \Delta \cong \mathbb{Z}_{2}$;
(3) $\Delta \in \operatorname{Ssat}^{\star}(G)$ e é maximal na ordem da inclusão.

c) As aplicações ${ }^{10}$

\footnotetext{
${ }^{10} X_{G}$ é o espaço de ordens de $G ; 3.18$.(a).
} 


$$
\sigma \in X_{G} \longmapsto \operatorname{ker} \sigma \in X(G) \quad \text { e } \Delta \in X(G) \longmapsto p_{\Delta}: G \longrightarrow G / \Delta
$$

são correspondências biunívocas e naturais, inversa uma da outra.

\section{Prova.}

a) Está claro que $(2) \Rightarrow(3) \Leftrightarrow(1)$. Se $-1 \in \Delta$, o axioma [SG2] em 3.1 e a saturação de $\Delta$ mostram que

$$
G=D_{G}(1,-1) \subseteq \Delta
$$

e $\Delta$ não é próprio.

b) (1) $\Rightarrow$ (2) vem do fato que $G / \Delta=\{1 / \Delta,-1 / \Delta\}$ tem apenas uma estrutura de grupo especial reduzido, aquela apresentada em 3.13 . É claro que $(2) \Rightarrow(1)$. A equivalência entre (1) e (3) vem da Proposição 2.10 em [DM1] que garante que $\Delta$ é próprio e maximal na ordem da inclusão sse $G=\Delta \sqcup-\Delta$.

c) É consequência de (b) e de 3.30.(b).

Lema 4.11. Sejam $f: G \longrightarrow G^{\prime}$ um morfismo em RSG e $\Delta^{\prime} \in \operatorname{Ssat}\left(G^{\prime}\right)$.

a) $\Delta^{\prime} \in \operatorname{Ssat}^{\star}\left(G^{\prime}\right) \quad \Rightarrow \quad f^{*}\left(\Delta^{\prime}\right) \in \operatorname{Ssat}^{\star}(G)$.

b) $\operatorname{card}\left(G / f^{*}\left(\Delta^{\prime}\right)\right) \leq \operatorname{card}\left(G^{\prime} / \Delta^{\prime}\right)$.

c) $\Delta^{\prime} \in \mathcal{F}\left(G^{\prime}\right) \quad \Rightarrow \quad f^{*}\left(\Delta^{\prime}\right) \in \mathcal{F}(G)$.

d) $\Delta^{\prime} \in X\left(G^{\prime}\right) \quad \Rightarrow \quad f^{*}\left(\Delta^{\prime}\right) \in X(G)$.

\section{Prova.}

a) Segue de 4.10.(a) : seja $\Delta^{\prime} \in S s a t^{\star}\left(G^{\prime}\right)$ então $-1 \notin \Delta^{\prime} ;$ como $f$ é $S G$-morfismo temos -1 $\notin f^{-1}\left[\Delta^{\prime}\right]=f^{*}\left(\Delta^{\prime}\right)$ e novamente por 4.10.(a) concluímos que $f^{*}\left(\Delta^{\prime}\right) \in S s a t^{\star}(G)$.

Os itens restantes são consequência de (a) e da observação, feita em 4.4, que

$$
f_{\Delta^{\prime}}: G / f^{*}\left(\Delta^{\prime}\right) \longrightarrow G^{\prime} / \Delta^{\prime}
$$

é injetor.

Lema 4.12. Sejam $f: G \longrightarrow G^{\prime}$ um morfismo sobrejetor em RSG $e \Delta \in \operatorname{Ssat}(G)$.

a) $\operatorname{card}\left(G^{\prime} / f_{*}(\Delta)\right) \leq \operatorname{card}(G / \Delta)$.

b) Se $f$ é regular ${ }^{11}$ e $\Gamma=\operatorname{ker} f$, então

$$
\Delta \in \operatorname{Ssat}_{\Gamma}(G) \Rightarrow \operatorname{card}\left(G^{\prime} / f_{*}(\Delta)\right)=\operatorname{card}(G / \Delta) .
$$

Em particular,

\footnotetext{
${ }^{11}$ Definida em 4.2.(e).
} 
$* \Delta \in \operatorname{Ssat}_{\Gamma}^{\star}(G) \quad \Leftrightarrow \quad f_{*}(\Delta) \in \operatorname{Ssat}^{\star}\left(G^{\prime}\right)$;

* $\Delta \in \mathcal{F}_{\Gamma}(G) \quad \Leftrightarrow \quad f_{*}(\Delta) \in \mathcal{F}\left(G^{\prime}\right)$;

$* \Delta \in X_{\Gamma}(G) \Leftrightarrow f_{*}(\Delta) \in X\left(G^{\prime}\right)$.

\section{Prova.}

a) Seja $\Delta \in S s a t(G)$; por 4.2.(a) temos

$$
\Delta \subseteq f^{*}\left(f_{*}(\Delta)\right)=\Sigma
$$

e portanto temos um SG-morfismo sobrejetor $p_{\Delta \Sigma}: G / \Delta \longrightarrow G / \Sigma$ que faz o primeiro diagrama em 4.6.(c) comutar. Como $f$ é sobrejetor, segue de 4.6.(e) que

$$
f_{f_{*}(\Delta)}: G / \Sigma \longrightarrow G^{\prime} / f_{*}(\Delta)
$$

é bijetor. Portanto, existe um SG-morfismo sobrejetor (a composição dos morfismos acima) de $G / \Delta$ em $G^{\prime} / f_{*}(\Delta)$ e o Axioma da Escolha garante que card $\left(G^{\prime} / f_{*}(\Delta)\right) \leq \operatorname{card}(G / \Delta)$ :

A prova de (b) é análoga, utilizando 4.6.(f), e nos permite concluir que, para cada $\Delta \in S_{s a t}(G)$ temos que $f_{f_{*}(\Delta)}: G / \Delta \longrightarrow G^{\prime} / f_{*}(\Delta)$ é $R S G$-isomorfismo.

A Proposição 4.2 e os Lemas 4.11 e 4.12 têm o seguinte Corolário, cuja prova omitimos.

Corolário 4.13. Sejam $G$ um grupo especial reduzido e $\Delta \in S \operatorname{sat}(G)$. Seja $p_{\Delta}: G \longrightarrow G / \Delta o$ $S G$-morfismo canônico.

a) Temos bijeções inversas, crescentes e naturais, $S$ sat $(G / \Delta) \rightleftarrows S$ sat $(G)$, dadas por:

$$
\left\{\begin{array}{l}
\left(p_{\Delta}\right)^{*}: S \operatorname{sat}(G / \Delta) \cong \operatorname{Ssat}_{\Delta}(G): \Gamma \in S \operatorname{sat}(G / \Delta) \mapsto p_{\Delta}^{*}(\Gamma) \in \operatorname{Ssat}_{\Delta}(G) ; \\
\left(p_{\Delta}\right)_{*}: \operatorname{Ssat}_{\Delta}(G) \cong \operatorname{Ssat}(G / \Delta): \Sigma \in \operatorname{Ssat}_{\Delta}(G) \mapsto\left(p_{\Delta}\right)_{*}(\Sigma)=\Sigma / \Delta \in \operatorname{Ssat}(G / \Delta) .
\end{array}\right.
$$

b) Estas bijeções se restrigem às correspondências biunívocas entre os seguintes conjuntos :

$$
* S_{s a t^{\star}}(G / \Delta) \rightleftarrows S s a t_{\Delta}^{\star}(G) ; \quad * \mathcal{F}(G / \Delta) \rightleftarrows \mathcal{F}_{\Delta}(G) ; \quad * X(G / \Delta) \rightleftarrows X_{\Delta}(G) .
$$

Observação 4.14. Seja $G$ um grupo especial reduzido.

a) Mencionamos em 3.18 que o espaço de ordens $X_{G}$, exatamente o conjunto dos SG-morfismos de $G$ em $\mathbb{Z}_{2}$, é um espaço Booleano.

Munindo o conjunto $X(G)$ da única topologia tal que as bijeções $X(G) \rightleftarrows X_{G}$, descritas em 4.10.(c), tornam-se homeomorfismos inversos, fazemos de $X(G)$ um espaço topológico Booleano. Deste modo, se $p, q \subseteq f$ definimos

$$
U_{p, q}=\{\Delta \in X(G): p \cup-q \subseteq \Delta\} .
$$

Então, o conjunto $\left\{U_{p, q}: p, q \subseteq_{f} G\right\}$ é uma base para a topologia contruída acima em $X(G)$. 
Se fixarmos $\Delta \in S \operatorname{sat}(G)$, então $X_{\Delta}(G) \subseteq X(G)$ é um subespaço fechado, constituindo portanto um espaço Booleano. Deste modo as bijeções $X(G / \Delta) \rightleftarrows X_{\Delta}(G)$ em 4.13.(a) tornamse homeomorfismos inversos.

b) Notamos que, com a topologias acima, obtemos para cada $S G$-morfismo $f: G \longrightarrow G^{\prime}$, funções contínuas :

$$
\begin{gathered}
X_{f}: X_{G^{\prime}} \longrightarrow X_{G}, \text { dada por } \sigma^{\prime} \mapsto \sigma^{\prime} \circ f \\
\text { e } \\
f^{*}: X\left(G^{\prime}\right) \longrightarrow X(G) \text { dada por } \Sigma^{\prime} \mapsto f^{-1}\left[\Sigma^{\prime}\right] .
\end{gathered}
$$

Claramente tratam-se de funtores isomorfos com valores na categoria $\mathbf{B S}$, dos espaços Booleanos e funções contínuas.

Encerramos esta seção tratando de propriedades conjuntísticas do reticulado completo dos subgrupos saturados de contém um saturado fixado. Lembramos que se $A$ é um conjunto

$$
2^{A} \quad \text { e } \quad 2_{f i n}^{A}
$$

indicam a família dos subconjuntos de $A$ e dos subconjuntos finitos de $A$, respectivamente.

Proposição 4.15. Sejam $G$ um grupo especial e $\Theta \in S$ sat $(G)$. Consideremos as funções

$$
\left\{\begin{array}{l}
(\text { i }) \quad 2^{X_{\Theta}(G)} \longrightarrow \text { Ssat }_{\Theta}(G) \quad \text { dada por } \quad S \mapsto \cap S \\
(\text { ii }) \text { Ssat }_{\Theta}(G) \longrightarrow 2^{X_{\Theta}(G)} \text { dada por } \Delta \mapsto X_{\Delta}(G) .
\end{array}\right.
$$

a) As funções cima são decrescentes e constituem uma conexão de Galois contravariante, isto é, para $\Delta \in S \operatorname{Sat}_{\Theta}(G)$ e $S \in 2^{X_{\ominus}(G)}$ temos

Em particular, $S \subseteq X_{\cap S}(G)$.

$$
\Delta \subseteq \cap S \Leftrightarrow S \subseteq X_{\Delta}(G)
$$

b) Para $\Delta \in S_{s a t}(G), \Delta=\bigcap X_{\Delta}(G)$.

c) $\mathcal{F}_{\Theta}(G)$ é filtro em $S$ sat $(G)$ e é o fecho por interseções finitas de $X_{\Theta}(G)$ em $S$ sat $(G)$. Em particular, temos que $\mathcal{F}_{\Theta}(G)$ é o filtro gerado por $X_{\Theta}(G)$ em $S$ sat $(G)$ e que $\left\langle\mathcal{F}_{\Theta}(G), \subseteq\right\rangle$ é um conjunto parcialmente ordenado dirigido para baixo.

d) A conexão de Galois contravariante descrita no item (a) $\left\langle 2^{X_{\Theta}(G)}, \subseteq\right\rangle \rightleftarrows\left\langle\right.$ ssat $\left._{\Theta}(G), \subseteq\right\rangle$ se restringe a uma outra: $\left\langle 2_{\text {fin }}^{X_{\Theta}(G)}, \subseteq\right\rangle \rightleftarrows\left\langle\mathcal{F}_{\Theta}(G), \subseteq\right\rangle$.

Prova. O item (a) segue imediatamente das definições, enquanto que (b) é consequência do Teorema de Separação em 3.30.(c).

c) O fato que $\mathcal{F}_{\Theta}(G)$ é um filtro vem das seguintes observações :

* Está claro que $\Delta \subseteq \Sigma$ e $\Delta \subseteq \mathcal{F}_{\Theta}(G) \Rightarrow \Sigma \in \mathcal{F}_{\Theta}(G)$

* Se $\Delta, \Sigma \in \mathcal{F}_{\Theta}(G)$, seja $\Gamma=\Delta \cap \Sigma$. Então $\Theta \subseteq \Gamma$ e é fácil verificar que a aplicação 


$$
a / \Gamma \in G / \Gamma \longmapsto\langle a / \Delta, a / \Sigma\rangle \in G / \Delta \times G / \Sigma
$$

é um SG-morfismo injetor. Portanto $\Gamma \in \mathcal{F}_{\Theta}(G)$, como necessário.

Dado $\Delta \in \mathcal{F}_{\Theta}(G)$, como $G / \Delta$ é reduzido, o Teorema de Separação em 3.30.(c) aplicado a $G / \Delta$ fornece

$$
\left\{1_{G / \Delta}\right\}=\bigcap X(G / \Delta)
$$

Como $G / \Delta$ é finito, temos que seu espaço de ordens também é finito $\left(X_{G / \Delta} \subseteq\left(\mathbb{Z}_{2}\right)^{G / \Delta}\right)$. Seja $X_{G / \Delta}=\left\{\tau_{1}, \ldots, \tau_{n}\right\}$ e considere, para $1 \leq i \leq n$,

$$
\sigma_{i}=\tau_{i} \circ p_{\Delta}
$$

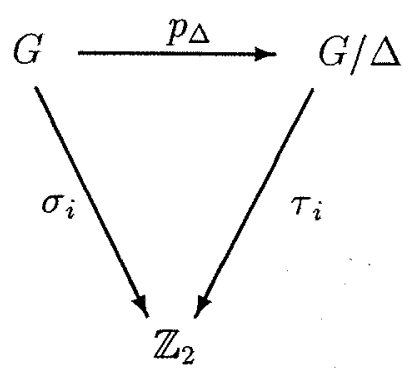

Então, $\left\{\sigma_{1}, \ldots, \sigma_{n}\right\} \subseteq X_{G}$ e claramente temos (3.30.(c))

$$
\Delta=\bigcap_{i=1}^{n} \operatorname{ker} \sigma_{i}
$$

Como ker $\sigma_{i} \in X_{\Theta}(G)$ (3.30.(b)), a igualdade acima mostra que $\Delta$ é interseção finita de elementos de $X_{\Theta}(G)$. Logo, $\mathcal{F}_{\Theta}(G)$ é o filtro gerado por $X_{\Theta}(G)$ em $S$ sat $(G)$.

A prova do item (d) é similar à prova realizada para (c) e será omitida. 


\section{Capítulo 5}

\section{Grupos Especiais Profinitos}

Neste Capítulo trataremos de grupos especiais profinitos: a existência destes limites projetivos na categoria dos grupos especiais, caracterizações destes objetos e sua ubiquidade entre os grupos especiais reduzidos: cada grupo especial reduzido é subgrupo especial, refletor de subformas, de um grupo especial reduzido profinito naturalmente associado àquele, e que merecerá:o título de envoltória profinita do grupo especial de partida.

A primeira seção do Capítulo contém alguns fatos básicos acerca de grupos topológicos, grupos Booleanos e grupos profinitos.

\subsection{Grupos Topológicos e Profinitos}

Como referências básicas para os temas desta seção recomendamos

* Para grupos topológicos, as seções 1 e 2 do Capítulo 3 em [Bou];

* Para grupos profinitos, as seções 2 e 3 do Capítulo 1 em [Rib].

Se $\langle G, \cdot 1\rangle$ é um grupo (escrito multiplicativamente) e $U, V \subseteq G$, utilizaremos notação standard para as translações, isto é,

$$
U \cdot V=\{u v: u \in U \text { e } v \in V\}
$$

Se $U=\{a\}$, indicamos $U \cdot V$ por $a V$; analogamente se $V=\{a\}$. Os grupos que serão de interesse neste trabalho são comutativos e neste caso $U \cdot V=V \cdot U$, para todo $U, V \in G$.

Definição 5.1. Um grupo topológico é um $\operatorname{par}\langle(G, \cdot, 1), T\rangle$, onde

* $(G, \cdot, 1)$ é um grupo;

* $T \subseteq 2^{G}$ é uma topologia em $G$;

* A função $G \times G \longrightarrow G$ dada por $\left\langle g, g^{\prime}\right\rangle \longmapsto g^{-1} \cdot g^{\prime}$ é função contínua, onde $G \times G$ possui a topologia produto.

Indicamos por $\nu_{1}$ o conjunto das vizinhanças abertas de $1 \mathrm{em} G$.

Um grupo topológico $\langle(G, \cdot, 1), T\rangle e^{\prime}$ 
* discreto se $T$ é a topologia discreta;

* Hausdorff se T é uma topologia Hausdorff;

* Booleano se $T$ é uma topologia Booleana, isto é, Hausdorff, compacta e com uma base constituída de conjuntos simultaneamente abertos e fechados ("clopen").

Indicamos por $\mathrm{Gr}^{t o p}$ a categoria cujos objetos são grupos topológicos e cujos morfismos são morfismos contínuos de grupos.

Teorema 5.2. Sejam $G$ e H grupos topológicos.

a) Para cada $g \in G$, multiplicação a direita e a esquerda por g são homeomorfismos. Em particular,

$$
T:=\left\{a U: a \in G, U \in \nu_{1}\right\}=\left\{U a: a \in q G e U \in \nu_{1}\right\} .
$$

b) Todo subgrupo ${ }^{1}$ de indice finito que é aberto ou fechado em $G$ é clopen.

c) Se $f: G \longrightarrow H$ é um morfismo de grupos, as seguintes condições são equivalentes :
(1) $f$ é contínua;
(2) $f$ é contínua no ponto 1 .

d) $\mathbf{G r}^{\text {top }}$ possui limites e colimites arbitrários. Em particular:

(1) Se $N$ é um subgrupo normal de $G$, o quociente $G / N$, munido da topologia quociente ${ }^{2}$ é um grupo topológico e a projeção natural $p_{N}: G \longrightarrow G / N$ é contínua.

(2) Se $G$ é grupo topológico e $G^{\prime} \subseteq G$ é um subgrupo algébrico de $G$ então $G^{\prime}$, munido da topologia de subespaço, torna-se um grupo topológico que é subgrupo topológico de $G$.

(3) Se $G_{i}, i \in I$, são grupos topológicos, o seu produto em $\mathrm{Gr}^{\text {top }}$ é o produto dos $G_{i}$, com a topologia produto.

(4) Se $\mathcal{C}$ é uma categoria pequena e $\mathcal{D}: \mathcal{C} \longrightarrow \mathbf{G r}^{\text {top }}$ é um diagrama, então o limite de $\mathcal{D}$ em $\mathrm{Gr}^{\text {top }}$ é o limite de $\mathcal{D}$ na categoria dos grupos, com a topologia de subespaço induzida pela topologia produto em $\prod_{A \in \mathrm{Ob}(\mathcal{C})} \mathcal{D}(A)$.

e) $G$ é discreto sse $\{1\}$ é aberto em $G$.

f) Se $G$ é finito então $G$ é Hausdorff sse é discreto sse é Booleano.

g) Se $G$ é um grupo topológico Booleano então $G$ é finito sse $G$ é discreto sse $\{\{1\}\}$ é sistema fundamental de vizinhanças abertas de $1 \in G$.

\section{Lema 5.3.}

a) Se G é um grupo topológico, as seguintes condiçốes são equivalentes:

\footnotetext{
${ }^{1}$ Não necessariamente normal.

${ }^{2}$ A topologia quociente é definida por : $V$ é aberto em $G / N$ sse $p_{N}^{-1}[V]$ é aberto em $G$.
} 
(1) Gé Hausdorff;

(2) $\{1\}$ é fechado em $G$;

(3) $\{1\}=\bigcap \nu_{1}$;

(4) Se $S \subseteq \nu_{1}$ é um sistema fundamental de vizinhaças de 1 , então $\bigcap S=\{1\}$.

b) Seja $\mathbf{G r}^{\text {Haus }}$ a subcategoria plena de $\mathbf{G r}^{\text {top }}$ constituída pelos grupos topológicos Hausdorffs. $\mathrm{Gr}^{\text {Haus }}$ é fechada em $\mathbf{G r}^{\text {top }}$ por limites arbitrários e quocientes por subgrupos normais fechados.

Mais explicitamente:

(1) Se $G$ é um grupo topológico Hausdorff e $N$ é um subgrupo normal fechado de $G$, o quociente $G / N$, munido da topologia quociente é um grupo topológico Hausdorff. Em particular, se $N$ é um subgrupo normal fechado (e/ou aberto) com indice finito então $G / N$ é discreto.

(2) Se $G$ é grupo topológico Hausdorff e $G^{\prime} \subseteq G$ é um subgrupo algébrico de $G$ então $G^{\prime}$ , munido da topologia de subespaço torna-se um grupo topológico Hausdorff que é subgrupo topológico de $G$.

(3) Se $G_{i}, i \in I$, são grupos topológicos Hausdorffs, o seu produto em $\mathbf{G r}^{\text {Haus }}$ é o produto dos $G_{i}$, com a topologia produto.

(4) Se $\mathcal{C}$ é uma categoria pequena e $\mathcal{D}: \mathcal{C} \longrightarrow \mathbf{G r}^{\text {Haus }}$ é um diagrama, então o limite de $\mathcal{D}$ em $\mathrm{Gr}^{\text {top }}$ é o limite de $\mathcal{D}$ na categoria dos grupos, com a topologia induzida pela topologia produto em $\prod_{A \in \mathrm{Ob}(\mathcal{C})} \mathcal{D}(A)$.

Algumas das propriedades fundamentais dos grupos Booleanos aparecem abaixo.

Teorema 5.4. Seja G um grupo topológico Booleano.

a) Existe um sistema fundamental de vizinhanças abertas de $1 \mathrm{em} G, S$, tal que todo $U \in S$ é um subgrupo normal de indice finito em $G$.

b) Sejam $\mathbf{G r}_{\text {fin }}$ a subcategoria plena de $\mathbf{G r}^{\text {top }}$ constituída pelos grupos finitos e discretos; $\mathbf{G} \mathbf{r}^{\text {bool }}$ a subcategoria plena de $\mathbf{G r}^{\text {top }}$ constituida pelos grupos Booleanos. Então, $\mathbf{G r}^{\text {bool }}$ contém $\mathbf{G r}_{\text {fin }} e$ é fechada em $\mathbf{G r}^{\text {top }}$ pelas seguintes construções :

(1) Isomorfismo;

(2) Quocientes por subgrupos normais e fechados, isto é, o quociente $G / N$ em 5.2.(c).(2)

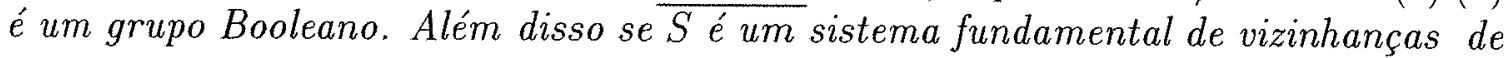
$1 \mathrm{em} G$, constituída de subgrupos normais de indice finito em $G$, então

$$
S_{N}=\{U \cdot N: U \in S\}
$$

é um sistema fundamental de vizinhanças de $1 \in G / N$, constituído de subgrupos normais de indice finito em $G / N$.

Além disso, se $f: G \longrightarrow H$ é um morfismo contínuo e denso de grupos booleanos então $f$ é sobrejetor, $N=k e r(f)$ é um subgrupo fechado de $G$ e o morfismo obtido de $f$ por passagem ao quociente $\bar{f}: G / N \longrightarrow H$ é um isomorfismo de grupos topológicos. 
(3) Subgrupos fechados.

(4) Produtos, isto é, o produto em 5.2.(c).(1) é um grupo Booleano. Além disso, se $S_{i}, i$ $\in I$, é um sistema fundamental de vizinhanças de $1 \in G_{i}$ como no item (a), então

$$
S=\left\{U \subseteq \prod_{i \in I} G_{i}: \begin{array}{c}
\exists J \subseteq f I \text { tal que } U=\left(\prod_{j \in J} U_{j}\right) \times\left(\prod_{i \notin J} G_{i}\right), \\
\operatorname{com} U_{j} \in S_{j}, j \in J
\end{array}\right\}
$$

é um sistema fundamental de vizinhanças abertas de 1 no produto, verificando as condiçôes em (a).

(5) Limites projetivos cofiltrantes, isto é, se $\langle I, \leq\rangle$ é um conjunto dirigido e $\mathcal{D}: I^{o p} \longrightarrow$ $\mathrm{Gr}^{\text {bool }}$ é um sistema cofiltrante de grupos Booleanos, o seu limite como em 5.2.(c).(3) é um grupo Booleano. Além disso, se $S_{i}$ é um sistema fundamental de vizinhanças de 1 em $G_{i}, i \in I$, verificando as condições em $(a)$, e $\lim \mathcal{D}=\widehat{G} \subseteq \prod_{i \in I} G_{i}$, então

$$
S=\left\{U \subseteq \widehat{G}: \begin{array}{c}
\exists J \subseteq f \text { tal que } U=\widehat{G} \cap\left(\left(\prod_{j \in J} U_{j}\right) \times\left(\prod_{i \notin J} G_{i}\right)\right) \\
\operatorname{com} U_{j} \in S_{j}, j \in J
\end{array}\right\}
$$

é um sistema fundamental de vizinhanças 1 em $\widehat{G}$, verificando as condições em (a).

O resultado fundamental abaixo relaciona grupos Booleanos e grupos profinitos :

Teorema 5.5. (Teorema 2.2, [Rib]) Se Gé um grupo topológico, as seguintes condiçôes são equivalentes:

(1) G é um grupo profinito, i.e., $G$ é um grupo topológico, isomorfo a um limite projetivo cofiltrante de grupos topológicos discretos e finitos.

(2) G é um grupo topológico Booleano.

Observamos que parte da prova deste resultado provém do Lema abaixo.

Lema 5.6. (Lema 2.5,[Rib]) Sejam:

* $(I, \leqslant)$ um conjunto ordenado dirigido;

* D : I $I^{o p} \longrightarrow$ Top $:(i \leqslant j) \in I \mapsto\left(X_{j} \stackrel{h_{j i}}{\longrightarrow} X_{i}\right)$ um diagrama cofiltrado de espaços topológicos;

* $\left(X \stackrel{h_{i}}{\longrightarrow} X_{i}\right)_{i \in I}$ um cone sobre o diagrama $D$ com vértice o espaço $X$.

Se, para cada $i \in I$, temos $h_{i}: X \rightarrow X_{i}$ contínua e sobrejetora então a flecha induzida pela propriedade universal, $h: X \longrightarrow{\underset{i \in I}{L}}_{i} X_{i}$ é uma função contínua que possui imagem densa. 
Veremos mais tarde (5.17) que o Teorema 5.5 possui um análogo para grupos especiais reduzidos.

Iremos precisar também de resultados envolvendo espaços uniformes. A referência mais completa sobre o tema permanece sendo [Bou]. Outras referências clássicas são [Tu] e [GJ], particularmente o Capítulo 15. Há uma apresentação singela e muito clara dos temas que iremos precisar nos Capítulos 6 e 7 de [Bu], que utilizaremos como referência para o Teorema 5.7.

Espaços uniformes constituem uma generalização dos espaços métricos, sendo o contexto adequado para as noções de continuidade uniforme e completude. Analogamente ao caso métrico, um espaço uniforme é completo se todo filtro de Cauchy é convergente (em geral, sequências não bastam).

A todo espaço uniforme $\langle X, \mathcal{U}\rangle$ está associado um espaço topológico, $\left\langle X, \tau_{\mathcal{U}}\right\rangle$, onde $\tau_{\mathcal{U}}$ é a topologia gerada pela uniformidade $\mathcal{U}$. Um espaço topológico $\langle X, \tau\rangle$ é uniformizável se existir uma uniformidade $\mathcal{U}$ em $X$ tal que $\tau=\tau_{\mathcal{U}}$. Por exemplo, são uniformizáveis :

* Espaços métricos, grupos topológicos e espaços vetoriais topológicos;

* Por um resultado clássico de Tychonoff, um espaço Hausdorff é uniformizável sse for completamente regular ${ }^{3}$

Os resultados necessários para o nosso trabalho estão sintetizados no seguinte

\section{Teorema 5.7 .}

a) Todo grupo topológico é uniformizável. Se $G, H$ são grupos topológicos e $f: G \longrightarrow H$ é um morfismo (algébrico) de grupos, então $f$ é contínuo sse for uniformemente contínuo.

b) Sejam $X, Y$ espaços uniformes e $D \subseteq X$ um subespaço denso de $X$. Seja $f: D \longrightarrow Y$ uma função uniformemente contínua. Se $Y$ é Hausdorff e completo, então $f$ tem uma única extensão a uma função uniformemente contínua $g: X \longrightarrow Y$.

c) Se $\langle X, \tau\rangle$ é um espaço Hausdorff compacto, existe uma única uniformidade $\mathcal{U}$ sobre $X$ tal que $\tau=\tau_{\mathcal{U}}$ e com esta uniformidade $X$ é um espaço uniforme completo.

d) Sejam $G, H$ grupos topológicos, com $H$ compacto e Hausdorff. Seja $S$ um subgrupo denso de $G$ e $f: S \longrightarrow H$ um morfismo (algébrico) de grupos que é contínuo na topologia induzida por $G$ em $S$. Então, $f$ tem uma única extensão a um morfismo contínuo de grupos, $g: G \longrightarrow H$.

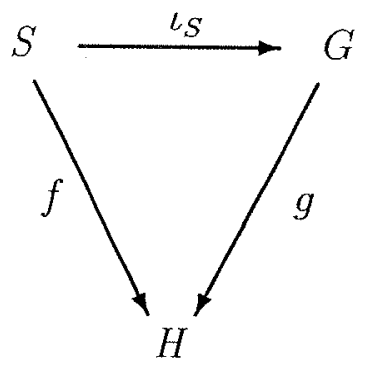

\footnotetext{
${ }^{3}$ Ponto e fechado disjuntos podem ser separados por função real contínua com valores em $[0,1]$; veja Teoremas 52 (pag. 123) e 53 (pag. 126) in [Bu].
} 
Prova. A primeira parte do item (a) vem do Teorema 47 (pag. 114) em [Bu]; o fato que um morfismo contínuo de grupos é uniformemente contínuo segue da definição de uniforme continuidade e da descrição explícita da uniformidade associada a um grupo topológico no resultado citado. O item (b) é o Teorema 61 (pag. 135) em [Bu]. O item (c) vem dos Teoremas 54 (pag. 126) e 65 (pag. 142) em [Bu].

Faremos a prova de (d), ilustrando um método que pode ser útil em outras ocasiões. Como $H$ é um espaço uniforme Hausdorff e completo (por (c)), $S$ é denso em $G$ e $f$ é uniformemente contínua (por (a)), seja $g: G \longrightarrow H$ a extensão uniformemente contínua de $f$ a $G$ (que existe por (b)). Vamos mostrar que $g$ é um morfismo de grupos. Como $1 \in S$, temos $g(1)=f(1)=1$. Consideremos as funções

$$
h_{1}, h_{2}: G \times G \longrightarrow H, \text { dadas por }\left\{\begin{aligned}
h_{1}(x, y) & =g(x)+g(y) ; \\
h_{2}(x, y) & =g(x+y) .
\end{aligned}\right.
$$

Note que $h_{1}, h_{2}$ são, respectivamente as composições

$$
G \times G \stackrel{g \times g}{\longrightarrow} H \times H \stackrel{+}{\rightarrow} H \text { e } G \times G \stackrel{\rightarrow}{\longrightarrow} \stackrel{g}{\longrightarrow} H
$$

sendo portanto contínuas. Seja $h: G \times G \longrightarrow H \times H$ definida por

$$
h(x, y)=\left\langle h_{1}(x, y), h_{2}(x, y)\right\rangle .
$$

Está claro que $h$ é contínua. Como $H$ é Hausdorff, a diagonal $\Delta=\{\langle u, u\rangle \in H \times H: u \in H\}$ é fechada em $H \times H$ e portanto

$$
\begin{aligned}
h^{-1}[\Delta] & =\left\{\langle x, y\rangle \in G \times G: h_{1}(x, y)=h_{2}(x, y)\right\} \\
& =\{\langle x, y\rangle \in G \times G: g(x+y)=g(x)+g(y)\}
\end{aligned}
$$

é fechado em $G \times G$. Como $g_{\mid S}=f$ é um morfismo de grupos, temos $S \times S \subseteq h^{-1}[\Delta]$. Já que $S$ é denso em $G$, o mesmo é verdade acerca de $S \times S$ em $G \times G ; \operatorname{assim} h^{-1}[\Delta]=G \times G$, mostrando que $g$ é um morfismo de grupos.

\subsection{Grupos Especiais Profinitos}

Devido à Proposição 10.11 em [DM1], as categorias SG e RSG não são indutivamente completas. Uma vez que ambas são categorias acessíveis, segue do Teorema 6.1.4 em [MP] (ou do Corolário 2.47 em [AR]) que não são projetivamente completas. Logo a existência de tipos particulares de limites projetivos constitui fato excepcional. Nesta seção, nos ocuparemos inicialmente da prova da existência de limites projetivos de grupos especiais finitos, generalizando construções em [Lim], realizadas na linguagem dos grupos especiais, e em [Mar4], estabelecidas na linguagem dos espaços de ordem abstratos; exploraremos adiante algumas propriedades básicas dos grupos especiais profinitos.

O Teorema 2.50 aplicado à linguagem $L_{S G}$ fornece

Teorema 5.8. Grupos especiais profinitos são retratos de ultraprodutos de grupos especiais fini- 
tos. Em particular, com a notação em 3.42 temos

a) O limite projetivo cofiltrante de grupos especiais finitos é um grupo especial, que é reduzido se cada componente do sistema cofiltrante o for.

b) Se $\widehat{G}$ é um grupo especial profinito e reduzido, então existe um corpo Pitagórico $F$ tal que $\widehat{G}$ é retrato, e portanto subgrupo puro, de $\mathcal{G}(F)$.

c) Todo grupo especial profinito e reduzido verifica a conjectura de Marshall (3.57).

Prova. Seja $\widehat{G}$ o limite projetivo de um sistema cofiltrante $G_{i}, i \in I$, de grupos especiais finitos. Pelo Teorema 2.50, $\widehat{G}$ é retrato de um ultraproduto dos $G_{i}$. Como o ultraproduto de grupos especiais é um grupo especial, que é reduzido se cada $G_{i}$ o for (2.23), e um retrato de um grupo especial (reduzido) é especial (respectivamente, reduzido), a conclusão em (a) segue imediatamente.

b) Pelos Teorema 2.1 e Lema 2.5 em [Cra], pela Brown-Marshall Inequality 10.10 em [Lam2 ${ }^{4}$, grupos especiais reduzidos finitos são isomorfos a grupos especiais provenientes de corpos Pitagóricos. Como ultraprodutos de grupos especiais reduzidos representáveis são representáveis (3.44.(b)), concluímos que existe um corpo Pitagórico $F$ tal que $\widehat{G}$ é retrato de $\mathcal{G}(F)$.Uma vez que $\widehat{G}$ é puro em $\mathcal{G}(F)$ e que $\mathcal{G}(F)$ verifica a conjectura de Marshall (por [DM2]), o item (c) segue do Lema 3.58 .

Uma primeira aplicação do Teorema 5.8 é o seguinte

Corolário 5.9. Seja $\langle I, \leq\rangle$ um conjunto parcialmente ordenado e dirigido e $\mathcal{G}: I^{o p} \longrightarrow \mathbf{R S G}$ um sistema cofiltrante tal que para todo $i \in I, G_{i}$ é um fan finito (3.41). Então, lim $\mathcal{G}$ é um fan.

Prova. É fácil verificar que todo subgrupo especial de um fan é um fan. Se $\widehat{G}=\lim \mathcal{G}$, o Teorema 5.8 garante que $\widehat{G}$ é um retrato de um ultraproduto dos $G_{i}$. Pela observação feita ao final de 3.41 , a família dos fans é definível por uma fórmula de primeira ordem em $L_{S G}$. Logo, o Teorema de Lós (2.23) implica que todo ultraproduto de fans é um fan. Como $\widehat{G}$ é retrato de um ultraproduto de fans (e portanto um subgrupo especial deste ultraproduto), concluímos que $\widehat{G}$ também é um fan.

A seguir, apresentaremos outra prova que o limite projetivo cofiltrante de grupos especiais finitos é um grupo especial, que fornecerá outras informações sobre a sua estrutura. O resultado que temos em mente é o Teorema 5.11 abaixo, que generaliza a Proposição $1.9 .8 \mathrm{em}$ [Lim], pois mostraremos que a inclusão natural do limite projetivo no produto reflete subformas, uma propriedade mais forte que completude. A prova segue o esquema geral apresentado em [Lim], que por sua vez origina-se em [KMS]. Para simplificar e homogeneizar a apresentação, introduzimos a. seguinte

\footnotetext{
${ }^{4}$ Ver também os Teoremas 4.2.1 e 4.2.2 em [Mar2].
} 
5.10. Notação. Sejam $\langle I, \leq\rangle$ um conjunto parcialmente ordenado e $\mathcal{G}: I^{o p} \longrightarrow$ PSG um $I^{o p}$-diagrama na categoria dos grupos pré-especiais. Para $i \leq j$ em $I$

* $G_{i}$ é o grupo pré-especial associado à $i$ por $\mathcal{G}$;

* $g_{j i}: G_{j} \longrightarrow G_{i}$ é o SG-morfismo associado ao par $\langle i, j\rangle$ por $\mathcal{G}$;

* $G=\prod_{i \in I} G_{i}$ é o grupo produto, com sua estrutura definida componente a componente; indicaremos por $\pi_{i}: G \longrightarrow G_{i}$ a projeção canônica.

Esta notação e seus análogos serão de uso constante no que segue.

Teorema 5.11. Sejam $\langle I, \leq\rangle$ um conjunto dirigido e $\mathcal{G}: I^{o p} \longrightarrow \mathbf{S G}$ um sistema cofiltrante de grupos especiais ${ }^{5}$, tal que para todo $i \in I, G_{i}$ é finito e discreto. Seja $\widehat{G}$ o limite projetivo de $\mathcal{G}$ na categoria dos conjuntos, isto é,

$$
\widehat{G}=\left\{\left\langle x_{i}\right\rangle_{i \in I} \in \prod_{i \in I} G_{i}: \forall i \leq j \text { em } I, g_{j i}\left(x_{j}\right)=x_{i}\right\}
$$

Sejam $\iota: \widehat{G} \longrightarrow G$ a inclusão de $\widehat{G}$ em $G=\prod_{i \in I} G_{i}$ e $g_{i}: \widehat{G} \longrightarrow G_{i}, i \in I$, os morfismos do cone limite projetivo $\left(g_{j}=\pi_{j} \circ \iota\right)$. Então:

a) Com a topologia induzida pela topologia produto em $G, \widehat{G}$ é um grupo topológico Booleano e para todo $i \in I, g_{i}$ é um morfismo contínuo de grupos topológicos.

b) Com a operação, as constantes e a isometria induzida por $G, \widehat{G}$ é um grupo especial e a aplicação Ł reflete subformas. Além disso, se cada $G_{i}$ é reduzido o mesmo é verdade para $\widehat{G}$.

c) Se $\psi$ é uma forma sobre $\widehat{G}$, então $D_{\hat{G}}(\psi)$ é fechado em $\widehat{G}$ e $D_{\hat{G}}(\psi) \stackrel{\cong}{\longleftrightarrow} \underset{i \in I}{\lim } D_{G_{i}}\left(g_{i} \star \psi\right)$.

d) $\widehat{G}$ verifica as seguintes propriedades:

(1) $S e G_{i}$ é reduzido, $i \in I$, a coleção

$$
S=\left\{U \subseteq \widehat{G}: \exists J \subseteq f \text { tal que } U=\widehat{G} \cap\left(\left(\prod_{j \in J}\left\{1_{G_{j}}\right\}\right) \times\left(\prod_{i \notin J} G_{i}\right)\right)\right\}
$$

é um sistema fundamental de vizinhanças de $1 \in \widehat{G}$, constituído de subgrupos saturados de indice finito em $\widehat{G}$;

(2) A relação de isometria, $\equiv_{\hat{G}} \subseteq \widehat{G}^{2} \times \widehat{G}^{2}$, é um subconjunto fechado de $\widehat{G}^{4}$ na topologia produto.

Prova. ${ }^{6}$ Seja $G=\prod_{i \in I} G_{i}$ o grupo especial produto. Como para $i \leq j$ em $I$, $g_{j i}$ é um morfismo de grupos especiais, está claro que as sequências constantes de valor 1 e -1 em $G$ (1 e -1 em $G$, respectivamente) estão em $\widehat{G}$; é imediato que com a operação de produto induzida por $G, \widehat{G}$ é um grupo de expoente 2 . Segue diretamente dos axiomas [SG0],.., [SG5] que a $L_{S G^{-}}$ subestrutura $\widehat{G} \hookrightarrow G$ é um subgrupo pré-especial de $G$. Como $G$ é reduzido se cada $G_{i}$ o for,

\footnotetext{
${ }^{5}$ Como em 1.39 e 1.41.(3).(II).

${ }^{6}$ A notação em 5.10 está em vigor.
} 
o mesmo será verdade para $\widehat{G}$. Além disso, com a topologia produto, $G$ é um grupo topológico Booleano (5.4.(b).(3)). Logo $\widehat{G}$, com a topologia induzida por $G$, é um grupo topológico.

Como os morfismos que acompanham o limite projetivo, $g_{i}: \widehat{G} \longrightarrow G_{i}$, são as restrições das projeções $\pi_{i}$ a $\widehat{G}\left(g_{i}=\pi_{i \mid \hat{G}}\right)$ está claro que estes também são morfismos contínuos de grupos topológicos.

Além disso, para $i \leq j \leq k$ em $I$, temos os diagramas comutativos na categoria $\mathbf{G r}^{t o p}$ :

(D)
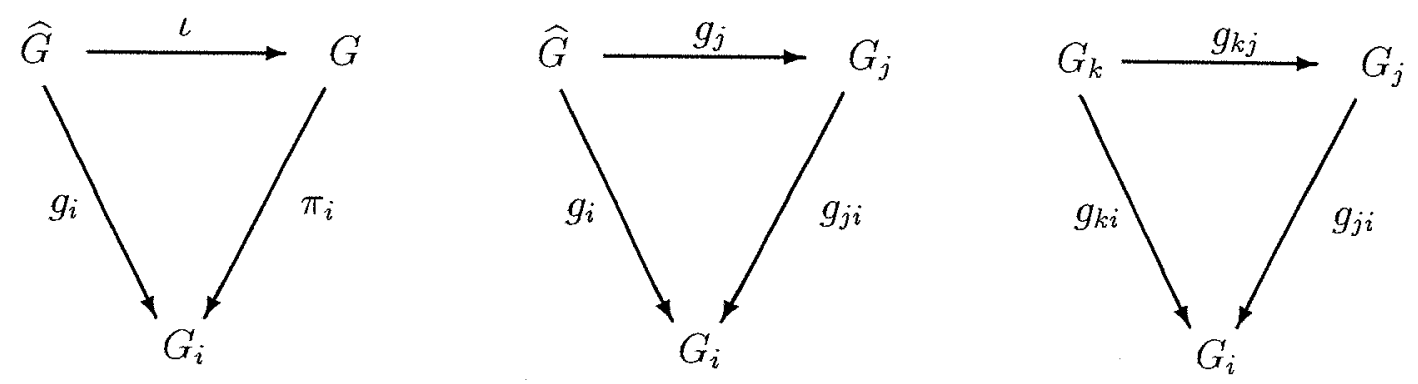

Temos o seguinte

Fato 5.12.

(1) Para $i \leq j$ em $I$, seja $G_{j i}=\left\{x \in G: g_{j i}\left(x_{j}\right)=x_{i}\right\}$. Então, $G_{j i}$ é um sugrupo fechado de $G$ e o seguinte diagrama é comutativo :

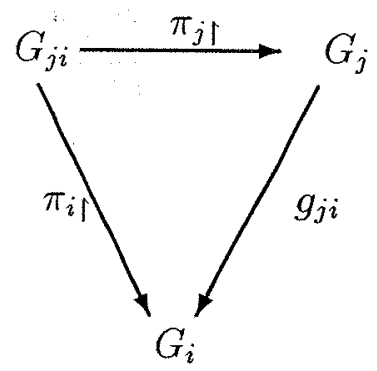

Além disso, $\widehat{G}=\bigcap\left\{G_{j i}: i \leq j\right.$ em $\left.I\right\}$; assim, $\widehat{G}$ é um subgrupo fechado de $G$ e um grupo topológico Booleano.

(2) Se $\varphi$ é uma $G$-forma, então $D_{G}(\varphi)$ é fechado em $G$.

(3) Para $n \geq 1$ e $\widehat{G}$-formas $\psi_{1}, \ldots, \psi_{n}$

$$
D_{G}\left(\psi_{1}\right) \cap \ldots \cap D_{G}\left(\psi_{n}\right) \neq \emptyset \Rightarrow \widehat{G} \cap D_{G}\left(\psi_{1}\right) \cap \ldots \cap D_{G}\left(\psi_{n}\right) \neq \emptyset .
$$

Prova.

(1) Está claro que $G_{j i}$ é um subgrupo de $G$ e que o diagrama do enunciado é comutativo. Se $x$ $\in G \backslash G_{j i}$, então $g_{j i}\left(x_{j}\right) \neq x_{i}$. Considere

$$
V=\left\{t \in G: t_{i}=x_{i} \text { e } t_{j}=x_{j}\right\}
$$

$V$ é aberto em $G$, pois $\left\{x_{i}\right\}$ e $\left\{x_{j}\right\}$ são abertos em $G_{i}$ e $G_{j}$. Como $V \cap G_{j i}=\emptyset$ temos que $G_{j i}$ é fechado em $G$. Claramente $\widehat{G}=\bigcap\left\{G_{j i}: i \leq j\right.$ em $\left.I\right\}$ e o restante da conclusão segue 
imediatamente.

(2) Seja $\varphi$ uma forma sobre $G$. Como a representação em $G$ é determinada componente a componente, temos

$$
D_{G}(\varphi)=\prod_{i \in I} D_{G_{i}}\left(\pi_{i} \star \varphi\right) .
$$

Como a topologia em $G_{i}$ é discreta e o produto de fechados nas componentes é fechado na topologia produto, segue imediatamente da igualdade acima que $D_{G}(\varphi)$ é fechado em $G$.

(3) Sejam $\psi_{1}, \ldots, \psi_{n}$ formas sobre $\widehat{G}$ tais que $\bigcap D_{G}\left(\psi_{k}\right) \neq \emptyset$. Por (b) temos que $\cap D_{G}\left(\psi_{k}\right)$ é fechado em $G$. Como $G$ é compacto, $G_{j i}$ é fechado em $G$ e $\widehat{G}=\bigcap\left\{G_{j i}: i \leq j\right.$ em $\left.I\right\}$ (por (a)), para mostrar que

$$
\widehat{G} \cap \cap D_{G}\left(\psi_{k}\right) \neq \emptyset
$$

é suficiente verificar que para para todo subconjunto finito $F \subseteq\{(i, j) \in I \times I: i \leq j\}$ temos

$$
\bigcap_{(i, j) \in F} G_{j i} \cap \cap D_{G}\left(\psi_{k}\right) \neq \emptyset
$$

Como $I$ é dirigido e $F$ é finito, existe $u \in I$ tal que para todo $(i, j) \in F, j \leq u$. Seja $C$ o conjunto dos elementos de $I$ que são componentes de algum elemento de $F$. Fixado $a \in \cap D_{G}\left(\psi_{k}\right)$, definimos $b \in G$ da seguinte forma, onde $v \in I$ :

$$
b_{v}= \begin{cases}a_{v} & \text { se } v \in I \backslash C \\ g_{u v}\left(a_{u}\right) & \text { se } v \in C .\end{cases}
$$

Note que para todo $v \in C$, a definição de $b$ garante que $b \in G_{u v}$. Se $(i, j) \in F$, a comutatividade do diagrama à direita em (D) fornece

$$
g_{j i}\left(b_{j}\right)=g_{j i}\left(g_{u j}\left(a_{u}\right)\right)=g_{u i}\left(a_{u}\right)=b_{i},
$$

mostrando que $b \in \bigcap_{(i, j) \in F} G_{j i}$. Por outro lado, seja $v \in I$; como a representação em $G$ é determinada coordenada a coordenada e $a \in \bigcap D_{G}\left(\psi_{k}\right)$, obtemos :

* Para $v \notin C$ temos $b_{v}=a_{v} \in \bigcap D_{G_{v}}\left(\pi_{v} \star \psi_{k}\right)$;

* Para $v \in C$, porque $g_{u v}$ é um SG-morfismo e $a_{u} \in \bigcap D_{G_{u}}\left(\pi_{u} \star \psi_{k}\right)$, temos,

$$
b_{v}=g_{u v}\left(a_{u}\right) \in \bigcap D_{G_{v}}\left(\left(g_{u v} \circ \pi_{u}\right) \star \psi_{k}\right),
$$

e como $\psi_{k} \in \widehat{G}$ temos $g_{u v} \circ \pi_{u} \star \psi_{k}=\pi_{v} \star \psi_{k}$ o que garante que, também neste caso, $b_{v} \in$ $\bigcap D_{G_{v}}\left(\pi_{v} \star \psi_{k}\right)$.

Assim, $b \in \bigcap_{(i, j) \in F} G_{j i} \cap \bigcap D_{G}\left(\psi_{k}\right)$, completando a prova do Fato.

Segue imediatamente do Fato 5.12.(3) e do Teorema 3.24 que $\iota$ reflete subformas e que $\widehat{G}$ é um grupo especial, estabelecendo (b).

c) Seja $\psi$ uma forma sobre $\widehat{G}$. Tendo em conta os dois primeiros diagramas em (D) (pag. 133), temos que para todo $i \leq j$ em $I$

$$
g_{j}=\pi_{j} \circ \iota \text { e } g_{i}=g_{j i} \circ g_{j} .
$$

Uma vez que $g_{j i}$ é um SG-morfismo, está claro que $g_{j i}$ leva $D_{G_{j}}\left(g_{j} \star \psi\right)$ em $D_{G_{i}}\left(g_{i} \star \psi\right)$. Deste 
modo, $\mathcal{G}$ induz um sistema cofiltrante

$$
\mathcal{G}_{\psi}=\left\langle D_{G_{i}}\left(\psi_{i}\right) ;\left\{g_{j i \uparrow}: i \leq j \mathrm{em} I\right\}\right\rangle,
$$

onde $\psi_{i}=g_{i} \star \psi=\left(\pi_{i} \circ \iota\right) \star \psi, i \in I$. Sabemos que

$$
\lim \mathcal{G}_{\psi}=\left\{z \in \prod_{i \in I} D_{G_{i}}\left(\psi_{i}\right): \forall i \leq j \mathrm{em} I, g_{j i}\left(z_{j}\right)=z_{i}\right\} \subseteq G .
$$

Se $x \in D_{\hat{G}}(\psi)$, como cada $g_{i}$ é um SG-morfismo, temos

$$
g_{i}(x)=\pi_{i}(\iota(x))=x_{i} \in D_{G_{i}}\left(\psi_{i}\right) .
$$

Além disso, já que $x \in \widehat{G}$, temos $g_{j i}\left(x_{j}\right)=x_{i}$, para todo $i \leq j \mathrm{em} I$. Logo, $x \in \prod_{i \in I} D_{G_{i}}\left(\psi_{i}\right) \mathrm{e}$ verifica a condição para pertencer a $\lim \mathcal{G}_{\psi}$ (ver $(\mathrm{I})$ acima), mostrando que

$$
D_{\hat{G}}(\psi) \subseteq \lim \mathcal{G}_{\psi} \text {. }
$$

Para a inclusão inversa, seja $z \in \lim \mathcal{G}_{\psi} ;$ então, para todo $i \leq j$ em $I$

$$
\text { (i) } g_{j i}\left(z_{j}\right)=z_{i} ; \quad \text { (ii) } z_{i} \in D_{G_{i}}\left(\psi_{i}\right) \text {. }
$$

A igualdade em (i) garante que $z \in \widehat{G}$. Pelo item (d) da Proposição 3.9, (ii) é equivalente a

$$
\forall i \in I,\left\langle-z_{i}\right\rangle \oplus \psi_{i} \text { é isotrópica em } G_{i} \text {. }
$$

Assim, $\langle-z\rangle \oplus \psi$ é uma $\widehat{G}$-forma que é isotrópica em $G=\prod_{i \in I} G_{i}$. Pelo item (b), Ł reflete subformas e portanto isotropia; logo, $\langle-z\rangle \oplus \psi$ é isotrópica em $\widehat{G}$. Uma nova aplicação de 3.9.(d), garante que $z \in D_{\hat{G}}(\psi)$, establecendo a igualdade desejada.

Para vermos que $D_{\hat{G}}(\psi)$ é fechado em $\widehat{G}$, seja $x \in \widehat{G} \backslash D_{\hat{G}}(\psi)$; pela igualdade estabelecida acima, isto significa que $x \notin \lim \mathcal{G}_{\psi}$, que por (I) é equivalente à existência de algum $i \in I$ tal que $x_{i} \notin D_{G_{i}}\left(\psi_{i}\right)$. Como $\left\{x_{i}\right\}$ é aberto em $G_{i}$, temos que

$$
V=\left\{z \in \widehat{G}: z_{i}=x_{i}\right\}
$$

é aberto em $\widehat{G}$; pelo argumento acima, vemos que $V \cap \widehat{G}$ é uma vizinhança aberta de $x$, disjunta de $D_{\hat{G}}(\psi)$, completando a prova de $(\mathrm{c})$.

d)

(1) Como para cada $i,\left\{1_{G_{i}}\right\}$ é subgrupo saturado e aberto em $G_{i}$ ( $G_{i}$ é reduzido e discreto), segue imediatamente do Fato 5.4.(b).(5) que $S$ é um sistema de vizinhaças abertas (e fechadas) de $1 \in \widehat{G}$, constituída de subgrupos saturados de índice finito.

(2) Como $\widehat{G}$ é subgrupo especial de $G$, para $x, y, u, v \in \widehat{G}$ temos

$$
\langle x, y\rangle \equiv_{\hat{G}}\langle u, v\rangle \text { sse }\langle x, y\rangle \equiv_{G}\langle u, v\rangle \text { sse } \forall i \in I,\left\langle x_{i}, y_{i}\right\rangle \equiv_{G_{i}}\left\langle u_{i}, v_{i}\right\rangle \text {. }
$$

A equivalência acima mostra que

$$
\equiv_{\hat{G}}=\widehat{G}^{4} \cap \prod_{i \in I} \equiv_{G_{i}}
$$

Como cada $G_{i}$ discreto, $\equiv_{G_{i}}$ é fechado em $G_{i}^{4}$. Uma vez que o produto de fechados é fechado na topologia produto, segue de (II) que $\equiv_{\hat{G}}$ é fechado em $\widehat{G}^{4}$ na topologia produto, encerrando a demonstração. 
Observação 5.13. O Teorema 5.11 vale para diagramas mais gerais que sistemas cofiltrantes, com as mesmas conclusões. Para explicar a generalização, lembramos a seguinte

Definição 5.13.A. Uma categoria $\mathcal{C}$ é filtrada se todo diagrama finito em $\mathcal{C}$ é base de um co-cone em $\mathcal{C}$. Dualmente, define-se a noção de cofiltrada.

Assim, se $\mathcal{C}$ é uma categoria filtrada e $F=\left\{A_{k} \stackrel{f_{k}}{\longrightarrow} B_{k}: 1 \leq k \leq n\right\}$ é um conjunto finito de morfismos em $\mathcal{C}$, existe um objeto $U$ e morfismos

$$
\alpha_{k}: A_{k} \longrightarrow U \text { e } \beta_{k}: B_{k} \longrightarrow U
$$

tais que para todo $j, k$ entre 1 e $n$

(i) $A_{k}=A_{j} \Rightarrow \alpha_{k}=\alpha_{j}$;

(ii) $B_{k}=B_{j} \Rightarrow \beta_{k}=\beta_{j}$;

(iii) O diagrama ao lado é comutativo :

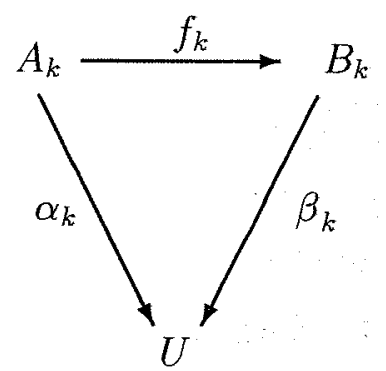

Está claro que $\mathcal{C}$ é filtrada sse $\mathcal{C}^{o p}$ é cofiltrada e que, se $\mathcal{C}$ é a categoria associada a um conjunto pré-ordenado $\langle I, \leq\rangle$ então $\mathcal{C}$ é filtrada sse $\langle I, \leq\rangle$ é dirigido.

O Teorema 5.11 pode então ser "generalizado", com exatamente as mesmas conclusões, substituindo-se "conjunto parcialmente ordenado e dirigido" por "categoria pequena e filtrada". O ponto central, é que a toda categoria filtrada $\mathcal{C}$ podemos associar um conjunto ordenado dirigido $\langle I(\mathcal{C}), \leqslant\rangle$ de modo que para toda categoria $\mathcal{A}$ e cada diagrama $D: \mathcal{C} \longrightarrow \mathcal{A}$ podemos associar um diagrama $D^{\prime}: I(\mathcal{C}) \longrightarrow \mathcal{A}$ de modo que as respectivas categorias dos co-cones são isomorfas. Omitiremos a prova deste resultado técnico, apenas recomendamos ao leitor interessado as referências $[\mathbf{G V}]$ e $[\mathbf{A R}]$.

Observação 5.14. Com a notação do enunciado e da prova do Teorema 5.11, suponhamos que $G_{i}$ é reduzido, $i \in I$. Consideremos

$$
T=\left\{\operatorname{ker} g_{i}: i \in I\right\} \text {. }
$$

Então, $T$ também é um sistema fundamental de vizinhanças abertas e fechadas de $1 \in \widehat{G}$, constituído de grupos saturados de índice finito. Primeiramente, como $g_{i}: \widehat{G} \longrightarrow G_{i}$ é um SG-morfismo e $G_{i}$ é reduzido e finito, está claro que ker $g_{i}$ é um subgrupo saturado de índice finito em $\widehat{G}$. Por outro lado, a continuidade de cada $g_{i}$ (5.11.(a)), garante que ker $g_{i}$ é aberto e fechado em $\widehat{G}$.

Para $i \in I$, como $g_{i}$ é a restrição da projeção $\pi_{i}$ a $\widehat{G}$, temos que

$$
\text { ker } g_{i}=\left\{x \in \widehat{G}: x_{i}=1\right\} \text {. }
$$


Assim, se $S$ é o sistema fundamental do item (d).(1) em 5.11, temos , $T \subseteq S$.

Se $U \in S$, existe um subconjunto finito $J$ de $I$ tal que $U$ é o conjunto dos elementos de $\widehat{G}$ cujas coordenadas em $J$ são iguais a 1 . Segue de $(*)$ que

$$
U=\bigcap_{j \in J} \operatorname{ker} g_{j}
$$

Como $I$ é dirigido, existe $k \in I$ tal que $j \leq k$, para todo $j \in J$. A comutatividade do diagrama do meio em (D) (pag. 133) garante que

$$
\text { ker } g_{k} \subseteq U \text {. }
$$

Uma vez que $S$ é um sistema fundamental de vizinhanças de $1 \in \widehat{G},\left(^{* * *}\right)$ acima mostra que o mesmo é verdade para $T$. Além disso, a discussão acima mostra que $S$ é o fecho por interseções finitas de $T$.

O item (d).(1) do Teorema 5.11 e a Observação 5.14 sugerem a seguinte

Definição 5.15. Seja $H$ um grupo topológico que é especial ${ }^{7}$. Dizemos que um conjunto $S$ é $u m$ pf-sistema em $H$ sse $S e ́$ um sistema fundamental de vizinhanças abertas de 1, constituído de subgrupos saturados de indice finito.

\section{Observação 5.16 .}

(i) É claro que se $H$ é um grupo topológico que é especial e que possui algum pf-sistema então $H$ admite o "maior" dos pf-sistema (na ordem da inclusão) $: \mathcal{V}(H) \doteq\{\Delta \in \mathcal{F}(H): \Delta$ é aberto em $H\}$.

(ii) Destacamos que se $H$ é um grupo topológico Hausdorff, que é especial, e que admite algum pf-sistema $S$ então $H$ é reduzido pois neste caso temos que $\left\{1_{H}\right\}=\bigcap S$ é um subgrupo saturado.

(iii) Se $H$ é um grupo especial reduzido discreto então $\mathcal{V}(H)=\mathcal{F}(H)$. Segue que $H$ admite pf-sistema sse existe $\Delta \in \mathcal{F}(H)$ tal que $\Delta \subseteq\{1\}$ sse $H$ é finito.

O análogo do Teorema 5.5 para grupos especiais reduzidos é o seguinte

Teorema 5.17. (Proposição 1.9.11, [Lim]) Seja H um grupo topológico que é especial e reduzido. Então as seguintes condições são equivalentes :

(1) $H$ é profinito, isto é, limite projetivo de grupos especiais reduzidos finitos e discretos;

(2) $H$ verifica as seguintes condições : 8

(i) H é um grupo topológico Booleano;

\footnotetext{
${ }^{7}$ As duas estruturas podem não estar relacionadas.

${ }^{8}$ Em [Lim], grupo especial profinito é definido pelas condições $(i),(i i)$ e (iii) abaixo.
} 
(ii) $\equiv_{H}$ é fechado em $H^{4}$ com a topologia produto;

(iii) Existe um pf-sistema em $H$ (5.15).

Observação 5.18. Seja $H$ um grupo topológico booleano que é especial.

a) Se $S$ é um pf-sistema em $H$, então $\langle S, \subseteq\rangle$ é um conjunto parcialmente ordenado, dirigido para baixo, pois $S$ é um sistema fundamental de vizinhanças de $1^{9}$. Assim para todo $U, V \in S$, existe $W \in S$, tal que $W \subseteq V \cap U$.

b) A prova de 5.17 mostra que se $H$ é um grupo especial reduzido que satisfaz as condições em 5.17.(2) e $S$ é um pf-sistema em $H$, então

$$
H \stackrel{\cong}{\longrightarrow} \lim _{\Sigma \in S} H / \Sigma
$$

onde os morfismos de conexão são os canônicos, isto é, para $\Delta \subseteq \Sigma$ em $S, p_{\Delta, \Sigma}$ é o único SG-morfismo que faz comutar o diagrama

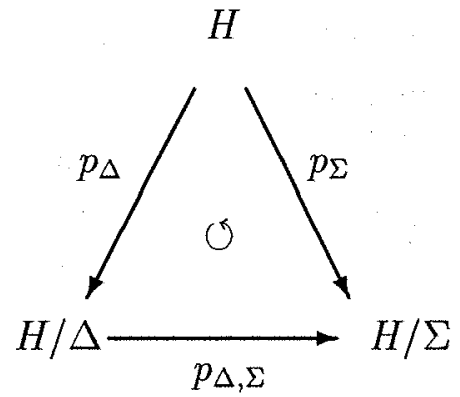

onde $p_{\Delta}: H \rightarrow H / \Delta$ e $p_{\Sigma}: H \rightarrow H / \Sigma$ são os SG-morfismos quocientes naturais.

Observação 5.19. Registramos que se $M$ é uma $L$-estrutura munida de uma topologia tal que:

* $M$ é um espaço compacto e Hausdorff;

* Todas as interpretações das $L$-operações em $M$ determinam funções contínuas;

* Todas as interpretações das $L$-relações em $M$ determinam subconjuntos fechados;

Então : ${ }^{10}$

* A cada $L_{M}$-termo $t\left(\bar{h}, v_{1}, \ldots, v_{n}\right)$ está associada uma função contínua $t_{\bar{h}}^{M}: M^{n} \longrightarrow M$;

* A cada fórmula em $\exists^{+}(L(M)) \varphi\left(\bar{h}, v_{1}, \ldots, v_{n}\right)$ está associado um subconjunto fechado de $M^{n}$ $[\varphi]_{M}=\left\{\bar{a} \in M^{n}: M=\varphi[\bar{h}, \bar{a}]\right\}$.

\footnotetext{
${ }^{9}$ Todo aberto contendo 1 contém um elemento de $S$.

${ }^{10}$ Ver 2.11 e 2.17 .
} 
Em particular, segue da caracterização 5.17 que a cada grupo especial profinito $H$ temos que para cada $H$-forma $\psi$, tem-se que $D_{H}(\psi) \subseteq H$ é fechado. ${ }^{11}$

Neste ponto, é natural considerarmos a seguinte

\section{Definição 5.20.}

(i) $\mathbf{R S G}^{\text {top }}$ é a categoria cujos objetos são os grupos topológicos que são especiais e reduzidos e com morfismos os RSG-morfismos contínuos.

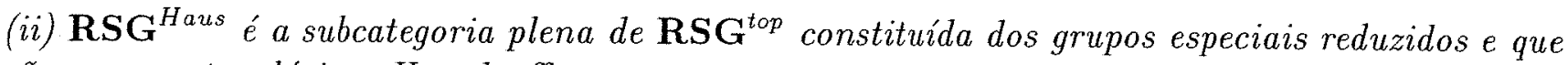
são grupos topológicos Hausdorffs.

(iii) $\mathbf{R S G}^{\text {disc }}$ é a subcategoria plena de $\mathbf{R S G}^{\text {top }}$ constituida dos grupos especiais reduzidos e que são grupos topológicos discretos.

(iv) $\mathbf{R S G}_{\text {fin }}$ é a subcategoria plena de $\mathbf{R S G}^{\text {top }}$ constituída dos grupos especiais reduzidos finitos e que são grupos topológicos discretos.

(v) $\mathbf{R S G}_{p f}$ é a subcategoria plena de $\mathbf{R S G}^{\text {top }}$ constituida dos grupos especiais reduzidos profinitos e que são grupos topológicos quando munidos da topologia booleana herdada.

\section{Observação $\mathbf{5 . 2 1}$.}

(i) Notamos que $\mathbf{R S G}_{f i n}$ é subcategoria plena de $\mathbf{R S G}_{p f}$ porque:

* Todo grupo especial finito $G$ é o limite projetivo cofiltrante do diagrama constante de valor $G$ sobre qualquer conjunto ordenado dirigido para baixo ;

* Um espaço topológico finito é discreto sse for booleano.

(ii) Como toda função entre espaços topológicos discretos é automaticamente contínua, podemos identificar as categorias $\mathbf{R S G}$ e $\mathbf{R S G}{ }^{\text {disc }}$ e considerar que $\mathbf{R S G}_{f i n}$ é subcategoria plena de $\mathbf{R S G}$.

A caracterização em 5.17 tem o seguinte

\section{Corolário 5.22. (Proposição 1.9.12, [Lim])}

a) A subcategoria $\mathbf{R S G}_{p f}$ é fechada em $\mathbf{R S G}^{\text {top }}$ por subgrupos especiais fechados, isto é, se $H$ é profinito e $H^{\prime}$ é um subgrupo especial de $H$, fechado na topologia de $H$, então, com a topologia induzida por $H, H^{\prime}$ é profinito.

b) A subcategoria $\mathbf{R S G}_{p f}$ é fechada em $\mathbf{R S G}^{\text {top }}$ por isomorfismos, produtos e limites projetivos cofiltrantes.

\footnotetext{
${ }^{11} \mathrm{~A}$ observação é portanto uma extensão do resultado provado em 5.11.(c) .
} 
As observações 5.14 e 5.18 têm consequências úteis. Antes de enunciá-las introduziremos a

5.23. Notação. Seja $\mathcal{G}=\left\langle G_{i}, g_{j i}\right\rangle$ um sistema cofiltrante sobre um conjunto dirigido $\langle I, \leq\rangle$ em $\mathbf{R S G}_{\text {fin }}$. Seja $\left\langle\widehat{G}, g_{i}\right\rangle=\lim \mathcal{G}$ o limite projetivo de $\mathcal{G}$. Para $i \leq j$ em $I$, definimos $* \widehat{G}_{i}=\widehat{G} / \operatorname{ker} g_{i}$ e $\mathfrak{p}_{i}: \widehat{G} \longrightarrow \widehat{G}_{i}$ o SG-morfismo quociente canônico ${ }^{12}$. Como ker $\mathfrak{p}_{i}=\operatorname{ker} g_{i}$, 5.14 implica que $T=\left\{\operatorname{ker} \mathfrak{p}_{i}: i \in I\right\}$ é um pf-sistema em $\widehat{G}$;

* $\widehat{g}_{i}: \widehat{G}_{i} \longrightarrow G_{i}$ o único SG-morfismo (injetor) que faz comutar o diagrama abaixo à esquerda $(3.29):$
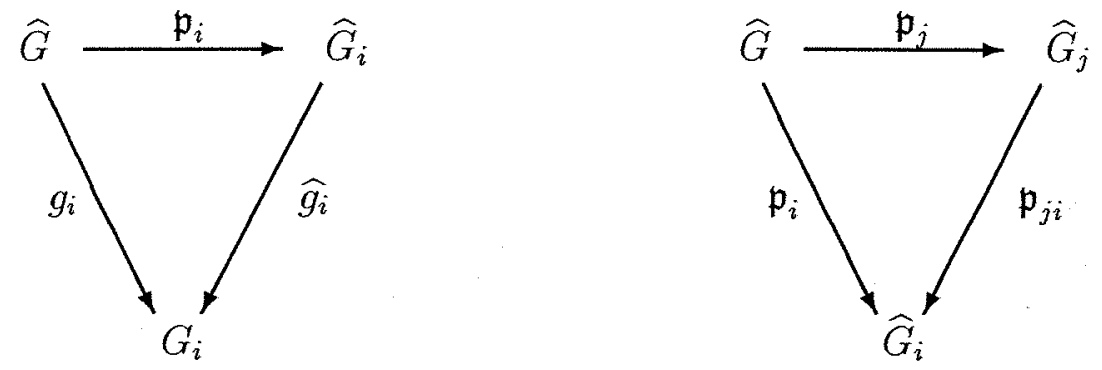

* Como ker $g_{j} \subseteq \operatorname{ker} g_{i}\left(\right.$ por $\left({ }^{* * *}\right)$ em 5.14), existe um único SG-morfismo, $\mathfrak{p}_{j i}: \widehat{G}_{j} \longrightarrow \widehat{G}_{i}$, que faz comutar o diagrama acima à direita.

É fácil verificar que para $i \leq j$ em $I$ o seguinte quadrado é comutativ :

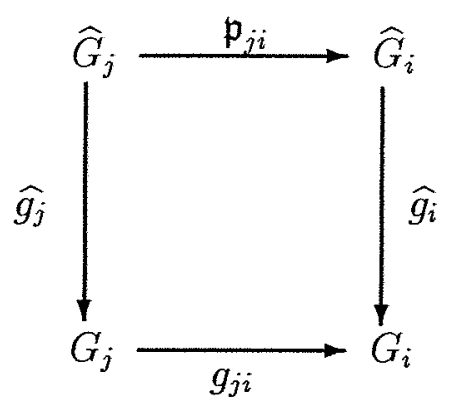

Além disso, como cada $\widehat{G}_{i}$ é finito e $\widehat{G}$ tem topologia booleana então $\widehat{G}_{i}$ é discreto com a topologia quociente induzida pelo o SG-morfismo quociente $\mathfrak{p}_{i}: \widehat{G} \longrightarrow \widehat{G}_{i}$.

Assim, $\left\langle\widehat{G}_{i}, \mathfrak{p}_{j i}\right\rangle$ é um sistema cofiltrante de $\langle I, \leq\rangle$ em $\mathbf{R S G}_{f i n}$.

Corolário 5.24. Sejam $\mathcal{G}=\left\langle G_{i}, g_{j i}\right\rangle$ um sistema cofiltrante sobre um conjunto dirigido $\langle I, \leq\rangle$ em $\mathbf{R S G}_{\text {fin }} e\left\langle\widehat{G}, g_{i}\right\rangle=\lim \mathcal{G}$ o limite projetivo de $\mathcal{G}$. Sejam $f: G \longrightarrow H$ um morfismo de grupos, onde $H$ é um grupo topológico, e $\mathcal{V}$ um sistema fundamental de vizinhaças de $1 \mathrm{em}$ H. Com a notação em 5.23 :

a) $f$ é contínuo sse para todo $U \in \mathcal{V}$ existe $i \in I$ tal que ker $\mathfrak{p}_{i}=\operatorname{ker} g_{i} \subseteq f^{-1}[U]$.

b) $\widehat{G}$ é isomorfo como grupo especial e topológico a $\lim \left\langle\widehat{G}_{i}, \mathfrak{p}_{j i}\right\rangle$.

\footnotetext{
${ }^{12}$ No lugar de p $_{\text {kerg }}$, como em 3.29.(c)
} 
c) Para $i \in I, g_{i}: \widehat{G} \longrightarrow G_{i}$ e $\mathfrak{p}_{i}: \widehat{G} \longrightarrow \widehat{G}_{i}$ são funções contínuas, onde $G_{i}$ e $\widehat{G}_{i}$ têm a topologia discreta.

Prova. Como registrado em 5.14

$$
T=\left\{\operatorname{ker} g_{i}: i \in I\right\}=\left\{\operatorname{ker} \mathfrak{p}_{i} i \in I\right\}
$$

é um pf-sistema em $\widehat{G}$. Assim, o item (a) é consequência de 5.2.(c), enquanto que o item (b) segue de 5.18. O item (c) está coberto por 5.11.(a) e 5.23.

Como estamos lidando com grupos especiais reduzidos e topológicos, uma questão natural é caracterizar os $S G$-carácteres contínuos, isto é, os SG-morfismos contínuos com valores em $\mathbb{Z}_{2}$ (com a topologia discreta).

Proposição 5.25. Seja $\mathcal{G}=\left\langle G_{i}, g_{j i}\right\rangle$ um sistema cofiltrante de grupos especiais finitos, reduzidos e discretos. Seja $\left\langle\widehat{G}, g_{i}\right\rangle=\lim \mathcal{G}$ e $\sigma \in X_{\hat{G}}$. Com a notação em 5.10 e 5.23 , considere as seguintes condições :

(1) Existem $i \in I$ e $\tau \in X_{G_{i}}$ tais que $\sigma=\tau \circ g_{i}$;

(2) $\sigma$ é contínuo;

(3) Existem $i \in I$ e $\mu \in X_{\hat{G}_{i}}$ tais que $\sigma=\mu \circ \mathfrak{p}_{i}$.

Então: $(1) \Rightarrow(2) \Leftrightarrow(3)$. Se para todo $i \in I, \widehat{g}_{i}: \widehat{G}_{i} \longrightarrow G_{i}$ é uma imersão completa, as condiçôes acima são equivalentes.

Prova. Uma vez que $g_{i}: \widehat{G} \longrightarrow G_{i}$ e $\mathfrak{p}_{i}: \widehat{G} \longrightarrow \widehat{G}_{i}$ são contínuas, onde $G_{i}$ e $\widehat{G}_{i}$ têm a topologia discreta (5.24.(c)), está claro que $(1) \Rightarrow(2)$ e que $(3) \Rightarrow(2)$.

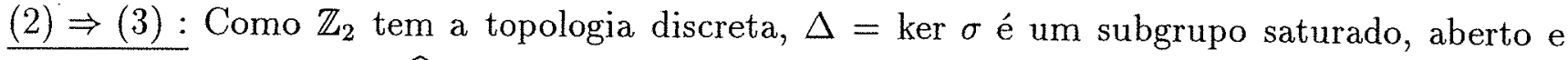
fechado, de índice $2 \mathrm{em} \widehat{G}$. Como

$$
T=\left\{\operatorname{ker} \mathfrak{p}_{i}: i \in I\right\}
$$

é um sistema fundamental de vizinhaças de 1 em $\widehat{G}(5.14)$, existe $i \in I$ tal que ker $\mathfrak{p}_{i} \subseteq \Delta$. Por 4.13.(b), $\mathfrak{p}_{i}[\Delta]$ é um subgrupo saturado de índice dois em $\widehat{G}_{i}$. Pelo Lema 3.30.(b), existe $\mu \in$ $X_{\hat{G}_{i}}$ tal que $\mathfrak{p}_{i}[\Delta]=\operatorname{ker} \mu$. Então, $\mu \circ \mathfrak{p}_{i}$ é um carácter contínuo de $\widehat{G}$, cujo núcleo contém $\Delta$. A maximalidade de $\Delta$ em $\widehat{G}$ imediatamente acarreta $\operatorname{ker}\left(\mu \circ \mathfrak{p}_{i}\right)=\Delta$ e portanto $\sigma=\mu \circ \mathfrak{p}_{i}$, como desejado.

Se $\widehat{g}_{i}: \widehat{G}_{i} \longrightarrow G_{i}$ é uma imersão completa, a Proposição 5.3 em [DM1] garante que todo $S G$-carácter de $\widehat{G}_{i}$ pode ser estendido a um SG-carácter em $G_{i}$, isto é, para todo $\mu \in X_{\hat{G}_{i}}$, existe $\tau \in X_{G_{i}}$ tal que $\tau \circ \widehat{g}_{i}=\mu$. Uma vez que $g_{i}=\widehat{g}_{i} \circ \mathfrak{p}_{i}$, segue imediatamente que $(3)^{\prime} \Rightarrow(1)$, completando a prova.

Proposição 5.26. Sejam $\mathcal{G}=\left\langle G_{i}, g_{j i}\right\rangle$ um sistema cofiltrante de grupos especiais finitos, reduzidos e discretos $e\left\langle\widehat{G}, g_{i}\right\rangle=\lim \mathcal{G}$. 
a) Se $Q$ é uma forma de Pfister de grau $\geq 1$ sobre $\widehat{G}$, então

$$
D_{\hat{G}}(Q)=\bigcap\left\{\Delta \in X(\widehat{G}): D_{\hat{G}}(Q) \subseteq \Delta \text { e } \Delta \dot{e} \text { um subgrupo aberto e/ou fechado }\right\} .{ }^{13}
$$

b) $O$ conjunto dos $S G$-carácteres contínuos de $\widehat{G}$ é denso no espaço de ordens, $X_{\hat{G}}$, de $\widehat{G}$.

\section{Prova.}

a) Seja $\Sigma=D_{\hat{G}}(Q)$. É suficiente (e necessário) mostrar que

$$
\forall a \notin \Sigma \text {, existe } \Delta \text { maximal, aberto e fechado, tal que } \Sigma \subseteq \Delta \text { e } a \notin \Delta .
$$

Notemos que, como uma forma de Pfister é isotrópica sse for hiperbólica (Proposição 2.2.(e), [DM1]) e que neste caso $D_{\hat{G}}(Q)=\widehat{G}$, podemos supor que $Q$ é anisotrópica; logo $D_{\hat{G}}(Q)$ é um subgrupo saturado próprio de $\widehat{G}$ (3.27.(d)), que também é fechado (5.11.(c)).

Se $a \notin \Sigma$, então $\langle a\rangle$ não é subforma de $Q$. Como a SG-imersão,

$$
\iota: \widehat{G} \longrightarrow G=\prod_{i \in I} G_{i}
$$

preserva subformas (5.11.(b)), $\langle\iota(a)\rangle$ não é subforma de $\iota \star Q$. Já que a isometria em $G$ é determinada coordenada a coordenada, existe $i \in I$ tal que

$$
\left\langle\left(\pi_{i} \circ \iota\right)(a)\right\rangle \text { não é subforma de }\left(\pi_{i} \circ \iota\right) \star Q .
$$

Como $g_{i}=\pi_{i} \circ \iota$, concluímos que $\left\langle g_{i}(a)\right\rangle=\left\langle a_{i}\right\rangle$ não é subforma de $g_{i} \star Q$, isto é,

$$
a_{i} \notin D_{G_{i}}\left(g_{i} \star Q\right) \text {. }
$$

Como cada $G_{i}$ é reduzido e $g_{i} \star Q$ é uma forma de Pfister sobre $G_{i}, 3.27$.(d) garante que $D_{G_{i}}\left(g_{i} \star Q\right)$ é um saturado em $G_{i}$. Pelo Teorema de separação (3.30.(c)), existe $\tau \in X_{G_{i}}$ tal que

$$
D_{G_{i}}\left(g_{i} \star Q\right) \subseteq \operatorname{ker} \tau \text { e } \tau\left(a_{i}\right)=-1
$$

Como $g_{i}: \widehat{G} \longrightarrow G_{i}$ é contínua $\left(G_{i}\right.$ com a topologia discreta), $\sigma=\tau \circ g_{i}$ é um SG-carácter contínuo de $\widehat{G}$, tal que

$$
\Sigma \subseteq \operatorname{ker} \sigma \text { e } \sigma(a)=-1
$$

Mas então, $\Delta=\operatorname{ker} \sigma$ é um subgrupo maximal saturado de índice 2, aberto e fechado (pois $\sigma$ é contínuo), que separa $\Sigma$ de $a$, como necessário.

b) É suficiente provar que todo aberto básico $\neq \emptyset \mathrm{em} X_{\hat{G}}$ possui um carácter contínuo. Por 3.18.(a), um aberto básico $U$ de $X_{\hat{G}}$ é da forma

$$
U=\left[a_{1}=1\right] \cap \ldots \cap\left[a_{n}=1\right], \operatorname{com}\left\{a_{1}, \ldots, a_{n}\right\} \subseteq \widehat{G}
$$

Consideremos a forma de Pfister $Q=\bigotimes_{i=1}^{n}\left\langle 1, a_{i}\right\rangle$; como $U \neq \emptyset$, o Princípio local global de Pfister (3.19) garante que $Q$ é anisotrópica ${ }^{14}$. Agora o item (a) fornece um SG-carácter contínuo de $\widehat{G}, \sigma$, tal que $D_{\hat{G}}(Q) \subseteq \operatorname{ker} \sigma$. Uma vez que $\left\{a_{1}, \ldots, a_{n}\right\} \subseteq D_{\hat{G}}(Q)$, concluímos que $\sigma\left(a_{j}\right)=1$, $1 \leq j \leq n$, mostrando que $\sigma \in U$ e completando a prova.

\footnotetext{
${ }^{13} X(G)$ é definido em 4.8.(c) .

${ }^{14} \mathrm{Se} Q$ fosse isotrópica seria hiperbólica (Prop. 2.2.(e), [DM1]); assim $Q \equiv_{G} 2^{n-1}\langle 1,-1\rangle$, onde $n \geq 1$ é o grau de $Q$. Por $3.19, \forall \sigma \in X_{\hat{G}}, \sigma(Q)=\sigma\left(2^{n-1}\langle 1,-1\rangle\right)=0$. Mas isto é impossível, pois para $\sigma \in U$ temos $\sigma(U)=2^{n} \neq 0$.
} 
Observação 5.27. Com a notação em 5.26, as provas de seus itens mostram que os SGcarácteres contínuos de $\widehat{G}$ dados por

$$
D=\bigcup_{i \in I}\left\{\tau \circ g_{i}: \tau \in X_{G_{i}}\right\}
$$

são densos em $X_{\hat{G}}$. Se

$$
E=\bigcup_{i \in I}\left\{\mu \circ \mathfrak{p}_{i}: \mu \in X_{\hat{G}_{i}}\right\}
$$

então $D \subseteq E$, pois para todo $i \in I, g_{i}=\widehat{g}_{i} \circ \mathfrak{p}_{i}$.

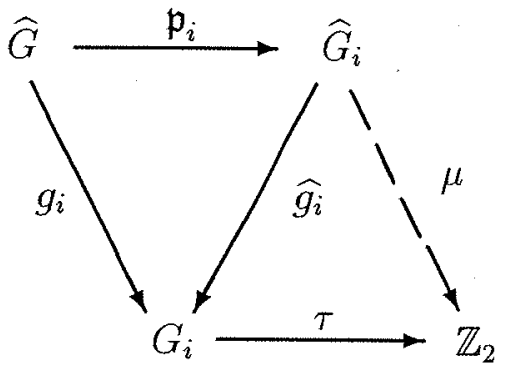

Assim, para todo $\tau \in X_{G_{i}}, \tau \circ \widehat{g}_{i} \in X_{\hat{G}_{i}}$. Logo $E$ também é denso em $X_{\hat{G}}$. Se $\widehat{g}_{i}$ é uma imersão completa, então $E=D$, pois todo $\mu \in X_{\hat{G}_{i}}$ é da forma $\tau \circ \widehat{g}_{i}$, para algum $\tau \in X_{G_{i}}$.

Temos agora condição de determinar para quais subgrupos fechados e saturados de um grupo especial Booleano o quociente é de novo um grupo especial Booleano.

Teorema 5.28. Seja $H$ um grupo especial Booleano reduzido e $\Sigma$ um subgrupo fechado e saturado de $H$. As seguintes condições são equivalentes : ${ }^{15}$

(1) Com a topologia quociente, $H / \Sigma$ é um grupo especial Booleano reduzido e a aplicação quociente canônica, $p_{\Sigma}: H \longrightarrow H / \Sigma$, é um $S G$-morfismo contínuo;

(2) $\Sigma$ é interseção dos subgrupos abertos e fechados de indice finito que o contém;

(3) $\Sigma$ é interseção dos subgrupos abertos e fechados de indice dois que o contém.

\section{Prova.}

$(1) \Rightarrow(2):$ Seja $\mathcal{V}$ um pf-sistema em $H / \Sigma$; se $U \in \mathcal{V}$ então $p_{\Sigma}^{*}(U)$ é um subgrupo aberto e fechado, saturado e de índice finito em $H$ (4.11.(c)). Como está claro que

$$
\Sigma=\bigcap\left\{p_{\Sigma}^{*}(U): U \in \mathcal{V}\right\},
$$

a conclusão de (2) segue imediatamente.

$(2) \Rightarrow(3)$ : Esta implicação é consequência imediata do seguinte

\footnotetext{
${ }^{15}$ Notemos que quocientes de grupos topológicos Booleanos por subgrupos normais fechados são grupos topológicos Booleanos, conforme 5.4.(b).(2) .
} 
Fato 5.29. Seja $\Delta$ um subgrupo saturado, aberto e fechado e de índice finito em $H$. Então, $\Delta$ é uma interseção finita de subgrupos abertos e fechados de índice 2 em $\mathrm{H}$.

Prova. Como $\Delta$ é aberto e fechado em $H$, o SG-morfismo quociente, $p_{\Delta}: H \longrightarrow H / \Delta$, é contínuo, onde $H / \Delta$ tem a topologia discreta, pois $p_{\Delta}^{-1}[\{1\}]=\Delta(5.2$.(e) e (c)). Como $H / \Delta$ é finito e reduzido, o Teorema da Separação (3.30.(c)) implica que

$$
\left\{1_{H / \Delta}\right\}=\bigcap_{i=1}^{n} \operatorname{ker} \sigma_{i}
$$

onde $X_{H / \Delta}=\left\{\sigma_{1}, \ldots, \sigma_{n}\right\}^{16}$. Uma vez que $p_{\Delta}$ é contínua, $\left\{\sigma_{1} \circ p_{\Delta}, \ldots, \sigma_{n} \circ p_{\Delta}\right\}$ é um subconjunto de SG-carácteres contínuos de $H$, tais que

$$
\Delta=\bigcap_{i=1}^{n} \operatorname{ker}\left(\sigma_{i} \circ p_{\Delta}\right) \text {. }
$$

Assim, $\operatorname{ker}\left(\sigma_{i} \circ p_{\Delta}\right), 1 \leq i \leq n$, são subgrupos abertos e fechados de índice 2 , cuja interseção é $\Delta$, completando a prova do Fato.

$(3) \Rightarrow(1):$ Seja

$$
\mathcal{M}=\left\{\Delta \in X_{\Sigma}(H): \Delta \text { é aberto e/ou fechado }\right\} \cdot{ }^{17}
$$

Nossas hipóteses garantem que $\Sigma=\bigcap \mathcal{M}$. É consequência imediata de 5.4.(b) que, com a topologia quociente, $H / \Sigma$ é um grupo Booleano e o SG-morfismo canônico, $\pi_{\Sigma}: H \longrightarrow H / \Sigma$, é contínuo. Devemos verificar, tendo em vista 5.17, que existe um pf-sistema em $H / \Sigma$ e que a relação de isometria é fechada em $(H / \Sigma)^{4}$ com a topologia produto. Começamos com o

Fato 5.30. Para cada $\mathcal{M} \subseteq\left\{\Delta \in X_{\Sigma}(H): \Delta\right.$ é aberto e/ou fechado $\}$ tal que $\Sigma=\bigcap \mathcal{M}$ temos:

a) Seja $N$ um subgrupo aberto e fechado de índice finito em $H$ tal que $\Sigma \subseteq N$. Então existe $\left\{\Delta_{1}, \ldots, \Delta_{n}\right\} \subseteq \mathcal{M}$ tal que

$$
\bigcap_{i=1}^{n} \Delta_{i} \subseteq \Sigma \cdot N
$$

b) $O$ conjunto

$$
T=\left\{\left(\Delta_{1} \cap \ldots \cap \Delta_{n}\right) / \Sigma: n \text { é um inteiro } \geq 1 \text { e }\left\{\Delta_{1}, \ldots, \Delta_{n}\right\} \subseteq \mathcal{M}\right\}
$$

é um pf-sistema em (5.15) em $H / \Sigma$ com a topologia quociente.

Prova.

a) Como

$$
N \subseteq \Sigma \cdot N=\bigcup_{x \in \Sigma} x N
$$

segue de 5.2.(a) que $\Sigma \cdot N$ é um subgrupo aberto de índice finito em $G$. Logo, por 5.2.(b), $\Sigma \cdot N$ é aberto e fechado em $G$. Se $\Sigma \cdot N=G$, nada a provar. Caso contrário, seja $K=G \backslash(\Sigma \cdot N)$, que é um compacto em $G$. Suponha, por absurdo, que para todo subconjunto finito $\mathcal{F}$ de $\mathcal{M}$ tivéssemos

\footnotetext{
${ }^{16}$ Note que o espaço de ordens de um grupo especial finito $G$ é finito pois $X_{G} \subseteq \mathbb{Z}_{2}{ }^{G}$.

${ }^{17} X_{\Theta}(G)$ é definido em 4.9.(d).
} 


$$
K \cap \bigcap \mathcal{F} \neq \emptyset
$$

Como $\mathcal{M}$ é uma família de fechados em $G$, a compacidade de $K$ garante que

$$
\emptyset \neq K \cap \cap \mathcal{M}=K \cap \Sigma,
$$

o que é impossível pois $\Sigma \subseteq \Sigma \cdot N$.

b) Por 5.4.(b).(2), se $S$ é um sistema fundamental de vizinhanças de 1 em $H$, constituído de subgrupos normais de índice finito em $H$, então o conjunto

$$
S_{\Sigma}=\{(\Sigma \cdot N) / \Sigma: N \in S\}
$$

é um sistema fundamental de vizinhanças de $1 \mathrm{em} H / \Sigma$, de subgrupos normais de índice finito. Agora note que se $\left\{\Delta_{1}, \ldots, \Delta_{n}\right\} \subseteq \mathcal{M}$, então :

* $\bigcap_{i=1}^{n} \Delta_{i}$ é saturado, de índice finito e aberto e fechado;

* $\left(\bigcap_{i=1}^{n} \Delta_{i}\right) / \Sigma$ é de índice finito em $H / \Sigma$, bem como aberto e fechado na topologia quociente ${ }^{18}$. Além disso, segue de 3.34 que $\left(\bigcap_{i=1}^{n} \Delta_{i}\right) / \Sigma$ é saturado em $H / \Sigma$.

Portanto, o item (a) garante que $T$ é um pf-sistema em $H / \Sigma$, como desejado. ${ }^{19}$

Resta provar que $\equiv_{H / \Sigma}$ é fechado em $(H / \Sigma)^{4}$.

Seja

$$
\alpha: H^{4} \longrightarrow(H / \Sigma)^{4}, \text { dada por } \alpha=p_{\Sigma} \times p_{\Sigma} \times p_{\Sigma} \times p_{\Sigma}
$$

Está claro que $\alpha$ é contínua e que a topologia produto em $(H / \Sigma)^{4}$ é a topologia quociente por $\alpha$ 20 , isto é,

$$
U \text { é aberto em }(H / \Sigma)^{4} \quad \Leftrightarrow \quad \alpha^{-1}(U) \text { é aberto em } H^{4} .
$$

Logo, para mostrar que $\equiv_{H / \Sigma}$ é fechado em $(H / \Sigma)^{4}$, é suficiente verificar que sua imagem inversa por $\alpha$ é fechada em $H^{4}$. Por 3.29.(a) temos que

$$
\langle a / \Sigma, b / \Sigma\rangle \equiv_{H / \Sigma}\langle c / \Sigma, d / \Sigma\rangle \quad \Leftrightarrow \quad \exists x, y, t, z \in \Sigma \text { tais que }\langle a x, b y\rangle \equiv_{H}\langle c t, d z\rangle .
$$

Assim,

$$
\alpha^{-1}\left(\equiv_{H / \Sigma}\right)=\left\{\langle a, b, c, d\rangle \in H^{4}: \exists x, y, t, z \in \Sigma \text { tais que }\langle a x, b y, t c, z d\rangle \in \equiv_{H}\right\} .
$$

Considere as funções

$$
g, h: H^{4} \times \Sigma^{4} \longrightarrow H^{4} \text { dadas por }\left\{\begin{array}{l}
g(\langle\langle a, b, c, d\rangle,\langle x, y, t, z\rangle\rangle)=\langle a x, b y, c t, d z\rangle \\
h(\langle\langle a, b, c, d\rangle,\langle x, y, t, z\rangle\rangle)=\langle a, b, c, d\rangle .
\end{array}\right.
$$

Uma vez que $H$ é um grupo topológico, $g$ é contínua; como os espaços em questão $(H$ e $\Sigma$ ) são Hausdorff e compactos ${ }^{21}$ e $h$ é a projeção que esquece as últimas quatro coordenadas, $h$ é

\footnotetext{
${ }^{18}$ Sua imagem inversa por $p_{\Sigma}$ é aberta e fechada.

${ }^{19}$ Segue desta prova que temos bijeção $\mathcal{V}_{\Sigma}(H) \rightleftarrows \mathcal{V}(H / \Sigma)$ onde $\mathcal{V}_{\Theta}(G)$ é a versão relativa de $\mathcal{V}(G)$, definido em 5.16.(i) .

${ }^{20}$ Temos isomorfismo contínuo de grupos topológicos compactos e Hausdorffs, $\bar{\alpha}: H^{4} / \Sigma^{4} \longrightarrow(H / \Sigma)^{4}, \operatorname{logo}$ $\bar{\alpha}$ é um homeomorfismo.

${ }^{21} \Sigma$ é fechado no Booleano $H$.
} 
contínua e fechada ${ }^{22}$. Agora observe que (I) implica

$$
\alpha^{-1}\left(\equiv_{H / \Sigma}\right)=h\left(g^{-1}\left(\equiv_{H}\right)\right)
$$

e como $\equiv_{H}$ é fechado em $H^{4}$, concluímos de (II) que o mesmo é verdade para $\alpha^{-1}\left(\equiv_{H / \Sigma}\right)$, encerrando a prova.

\section{Observação 5.31.}

(i) Notando que quocientes de grupos topológicos Hausdorffs por subgrupos normais fechados e/ou abertos e com índices finitos são grupos topológicos finitos e discretos (5.3.(b).(1)) vemos imediatamente que as condições (2) e (3) do Teorema 5.28 são sempre equivalentes, mesmo quando tomamos $H$ um grupo topológico Hausdorff que é especial e reduzido $\left(H \in \mathbf{R S G}^{\text {Haus }}\right)$.

(ii) Se $H$ um grupo reduzido especial que é topológico e Hausdorff e $\Sigma \subseteq H$ um subgrupo saturado e fechado então, claramente, $\Sigma$ satisfaz a condição (2) de 5.28 sse

[pst] Para cada $h \notin \Sigma$ existe $\Delta \in X(H)$ tal que $h \notin \Delta \mathrm{e}$

$$
\Sigma \subseteq \Delta \quad \text { e } \Delta \subseteq H \text { é aberto . }
$$

Quando este for o caso, diremos que $\Sigma$ possui a propriedade de separação topológica.

(iii) Se $H \in \mathbf{R S G}_{p f}$ e $\Sigma \subseteq H$ é um subgrupo saturado e fechado com a [pst] temos, pelo Teorema 5.28 que $H / \Sigma \in \mathbf{R S G}_{p f}$; além disso, segue dos Fatos 5.29 e 5.30 que, se $\mathcal{N} \subseteq\left\{\Delta \in \mathcal{F}_{\Sigma}(H): \Delta\right.$ é aberto e/ou fechado $\}$ é um conjunto ordenado dirigido para baixo tal que $\Sigma=\bigcap \mathcal{N}$ então $\{\Delta / \Sigma: \Delta \in \mathcal{N}\}$ é um pf-sistema de $H / \Sigma$ e portanto, pela Observação 5.18.(b):

$$
H / \Sigma \stackrel{\cong}{\longrightarrow} \lim _{\Delta \in \mathcal{N}}^{\longleftarrow}(H / \Sigma) /(\Delta / \Sigma) \stackrel{\cong}{\longrightarrow} \lim _{\Delta \in \mathcal{N}} H / \Delta .
$$

(iv) Se $H \in \mathbf{R S G}^{\text {Haus }}$ então a todo subconjunto $Y \subseteq H$ podemos associar $\widehat{Y}$, o menor subgrupo saturado e fechado de $H$ que satisfaz [pst] e que contém $Y$. Pois de fato temos

$$
\widehat{Y}=\bigcap\{\Delta \in \mathcal{F}(H): Y \subseteq \Delta \text { e } \Delta \text { é aberto }\}=\bigcap\{\Delta \in X(H): Y \subseteq \Delta \text { e } \Delta \text { é aberto }\} .
$$

Em particular, segue do Teorema 5.28 acima que se $G$ é um grupo especial reduzido e profinito e $\Theta \subseteq G$ é um subgrupo saturado e fechado de $G$ então o quociente de $G$ pela "regularização separada de $\Theta$ " , $G / \widehat{\Theta}$ é o maior quociente de $G / \Theta \in \mathbf{R S G}^{\text {Haus }}$ que é um grupo especial reduzido e profinito $\left(G / \widehat{\Theta} \in \mathbf{R S G}_{p f}\right)$.

A Proposição 5.26.(a) e o Teorema 5.28 fornecem

Corolário 5.32. Se $Q=\bigotimes_{i=1}^{n}\left\langle 1, a_{i}\right\rangle$ é um forma de Pfister sobre um grupo especial Booleano reduzido $G$ e $\Delta=D_{G}(Q)$, então, com a topologia quociente, $G / \Delta$ é um grupo especial Booleano reduzido.

\footnotetext{
${ }^{22} \mathrm{Em}$ geral, a projeção que esquece coordenadas não é fechada.
} 
O último tema desta seção é o processo de troca de base, que permite ligar dois sistemas cofiltrantes sobre bases distintas. As noções que introduziremos a seguir são um caso particular de um esquema geral de troca de base ${ }^{23}$. No entanto, pensamos ser mais adequado, inclusive para facilitar a leitura, discutirmos o assunto no contexto em que será utilizado.

Definição 5.33. Sejam $\langle I, \leq\rangle$ e $\langle L, \leq\rangle$ conjuntos parcialmente ordenados e dirigidos. Sejam

$$
\mathcal{G}=\left\langle G_{i} ; g_{j i}\right\rangle \text { e } \mathcal{H}=\left\langle H_{l} ; h_{m l}\right\rangle
$$

sistemas cofiltrantes de grupos especiais sobre $I$ e $L$, respectivamente. Um morfismo de troca de base, $\alpha: \mathcal{G} \longrightarrow \mathcal{H}$, consiste de

* Uma função crescente, $\alpha: L \longrightarrow I$;

* Para cada $l \in L$, um SG-morfismo, $f_{l}: G_{\alpha(l)} \longrightarrow H_{l}$, tal que para todo $l \leq m$ em L o seguinte diagrama é comutativo:

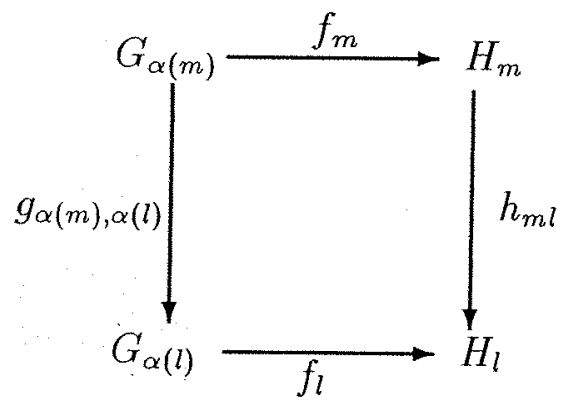

Indicamos por $I d_{\mathcal{G}}$ o morfismo de troca de base dado por $\left\langle I d_{I}, I d_{G_{i}}\right\rangle$. Está claro que a composição natural de morfismos de troca de base ${ }^{24}$ é um morfismo de troca de base.

Proposição 5.34. Sejam $\langle I, \leq\rangle,\langle L, \leq\rangle$ e $\langle P, \leq\rangle$ conjuntos parcialmente ordenados e dirigidos. Sejam

$$
\mathcal{G}=\left\langle G_{i} ; g_{j i}\right\rangle, \quad \mathcal{H}=\left\langle H_{l} ; h_{m l}\right\rangle \text { e } \mathcal{K}=\left\langle K_{p} ; k_{p q}\right\rangle
$$

sistemas cofiltrantes de grupos especiais discretos e finitos sobre $I, L$ e $P$, respectivamente. Sejam

$$
\alpha: \mathcal{G} \longrightarrow \mathcal{H} \text { e } \beta: \mathcal{H} \longrightarrow \mathcal{K}
$$

morfismos de troca de base e sejam

$$
\left\langle\widehat{G}, g_{i}\right\rangle=\lim \mathcal{G},\left\langle\widehat{H}, h_{l}\right\rangle=\lim _{\leftarrow} \mathcal{H} \quad \text { e }\left\langle\widehat{K}, k_{p}\right\rangle=\lim \mathcal{K}
$$

os limites projetivos correspondentes. Então:

a) Existe um único $S G$-morfismo contínuo, $f_{\alpha}: \widehat{G} \longrightarrow \widehat{H}$, tal que para todo $l \in L$ o seguinte diagrama é comutativo :

\footnotetext{
${ }^{23}$ Conforme a seção 1.2 .5 .

${ }^{24}$ Compor as funções de base e as de fibra correspondentes.
} 


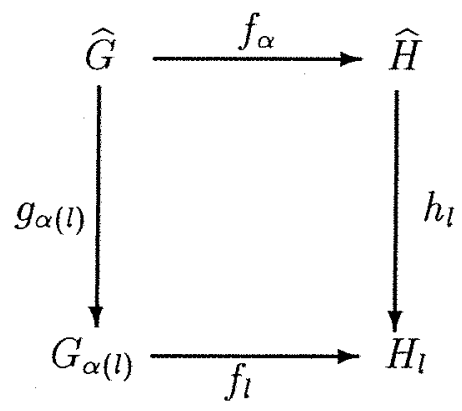

b) $f_{(\beta \circ \alpha)}=f_{\beta} \circ f_{\alpha}$. Se $\alpha=I d_{\mathcal{G}}$, então $f_{\alpha}=I d_{\hat{G}}$.

Prova. Notemos que, como nossos sistemas cofiltrantes são de grupos especiais finitos e discretos, todos os morfismos de troca de base são compostos por $S G$-morfismos contínuos.

a) Para cada $l \in L$, consideremos o SG-morfismo contínuo

$\omega_{l}: \widehat{G} \longrightarrow H_{l}$, dado por $\omega_{l}=f_{l} \circ g_{\alpha(l)}$

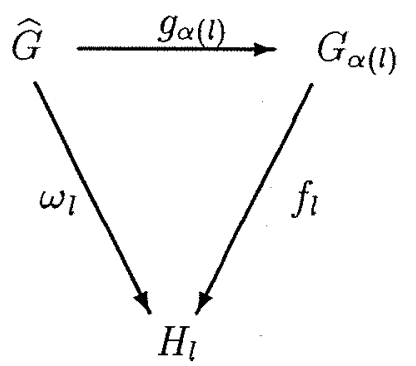

Então, $\left\langle\widehat{G}, \omega_{l}\right\rangle$ é um cone sobre $\mathcal{H}$, isto é, para todo $l \leq m$ em $L$, o diagrama abaixo à esquerda é comutativo :
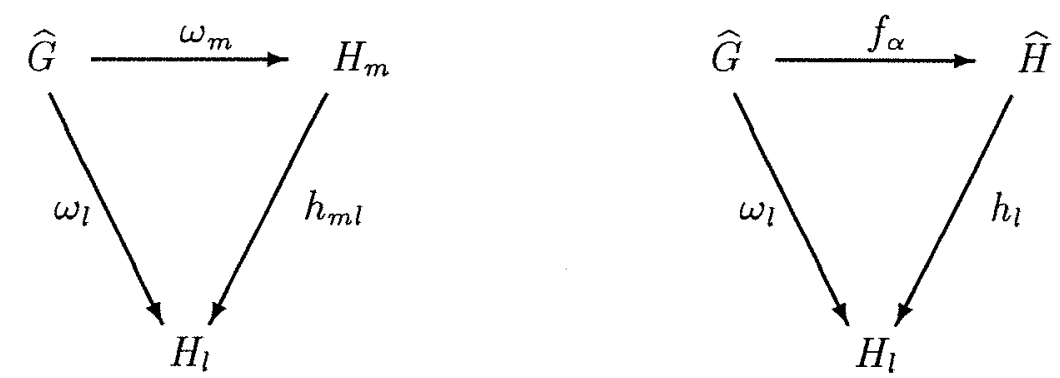

De fato, lembrando o diagrama comutativo na Definição 5.33, juntamente com o fato que $g_{\alpha(l)}=g_{\alpha(m), \alpha(l)} \circ g_{\alpha(m)}$, obtemos

$$
h_{m l} \circ \omega_{m}=h_{m l} \circ f_{m} \circ g_{\alpha(m)}=f_{l} \circ g_{\alpha(m), \alpha(l)} \circ g_{\alpha(m)}=f_{l} \circ g_{\alpha(l)}=\omega_{l},
$$

como necessário.

Segue do Teorema 5.11 que $\widehat{H}=\lim \mathcal{H}$ é limite projetivo na categoria $\mathbf{R S G}^{\text {top }}$, dos grupos especiais reduzidos topológicos e $R S G$-morfismos contínuos, portanto existe um único SG-morfismo contínuo, $f_{\alpha}: \widehat{G} \longrightarrow \widehat{H}$, tal que para todo $l \in L$ o diagrama a direita em (D) acima é comutativo; em particular temos

$$
h_{l} \circ f_{\alpha}=\omega_{l}=f_{l} \circ g_{\alpha(l)}
$$


mostrando que o quadrado do enunciado é comutativo.

Disponibilizamos abaixo uma verificação explícita que o $S G$-morfismo $f_{\alpha}$ é contínuo:

Lembrando que por 5.14

$$
T(\widehat{G})=\left\{\operatorname{ker} g_{i}: i \in I\right\} \quad \text { e } T(\widehat{H})=\left\{\operatorname{ker} h_{l}: l \in L\right\}
$$

são pf-sistemas em $\widehat{G}$ e $\widehat{H}$, respectivamente, é suficiente mostrar (por 5.2.(c)) que para todo $l \in L$, existe $i \in I$ tal que ker $g_{i} \subseteq f_{\alpha}^{-1}\left(\right.$ ker $\left.h_{l}\right)$. Mas a comutatividade do quadrado no enunciado, do diagrama a direita em (D) e a definição de $\omega_{l}$ garantem que

$$
\operatorname{ker} g_{\alpha(l)} \subseteq g_{\alpha(l)}^{-1}\left(f_{l}^{-1}(1)\right)=\omega_{l}^{-1}(1)=f_{\alpha}^{-1}\left(\operatorname{ker} h_{l}\right) \text {, }
$$

O item (b) é rotineiro e omitiremos a sua demonstração.

\subsection{A Envoltória Booleana de um Grupo Profinito Re- duzido}

Nesta seção determinaremos a envoltória Booleana de um grupo especial profinito (3.4.3) ${ }^{25}$. Para isto precisaremos registrar um resultado acerca de álgebras de Boole completas, o Lema 5.37 abaixo, que provavelmente é conhecido, mas para o qual não foi encontrada referência na literatura. A notação para álgebras de Boole está sumarizada em 3.4.3.

Definição 5.35. Uma álgebra de Boole $B$ é completa se para todo $S \subseteq B, \bigvee S$ e $\bigwedge S$ existem em B. Um morfismo de álgebras de Boole, $f: A \longrightarrow B$, é completo se para todo $S \subseteq A$

$$
f(\bigvee S)=\bigvee f(S) \text { e } f(\bigwedge S)=\bigwedge f(S)
$$

Observação 5.36. Seja $B$ uma álgebra de Boole.

a) Como em todo reticulado, as seguintes condições são equivalentes:

$* \forall S \subseteq B$, existe $\bigvee S \in B$

$* \forall S \subseteq B$, existe $\wedge S \in B$.

b) Se $B$ é completa, como as leis de de Morgan são válidas em $B$, isto é,

$$
-(\bigvee S)=\bigwedge_{s \in S}-s \text { e }-(\bigwedge S)=\bigvee_{s \in S}-s
$$

está claro que um Ba-morfismo preserva $\bigvee$ sse preserva $\Lambda$.

c) É fácil verificar que se $B_{i}, i \in I$, são álgebras de Boole completas e $A=\prod_{i \in I} B_{i}$ tem a estrutura produto, $A$ é uma álgebra de Boole completa e as projeções coordenadas são Bamorfismos completos.

\footnotetext{
${ }^{25}$ A referência básica acerca da envoltória Booleana de um grupo especial reduzido é o Capítulo 4 em [DM1].
} 
Lema 5.37. Seja $\mathcal{I}$ uma categoria pequena e $\mathcal{B}=\left\langle B_{i} ; f_{\alpha}\right\rangle$ diagrama sobre $\mathcal{I}$ constituido de álgebras de Boole e Ba-morfismos. Seja

$$
\left\langle\widehat{B}, f_{i}\right\rangle=\lim \mathcal{B}
$$

o limite projetivo de $\mathcal{B}$.

a) $\widehat{B}$ é uma álgebra de Boole e $f_{i}$ é um Ba-morfismo, para todo $i \in I=\operatorname{Obj}(\mathcal{I})$.

b) Se $B_{i}$ é completa e os morfismos $f_{\alpha}$ são completos, para cada flecha $(i \stackrel{\alpha}{\rightarrow} j)$ em $\mathcal{I}$, então $\widehat{B}$ é uma álgebra de Boole completa e $f_{i}$ são Ba-morfismos completos, $i \in I=\operatorname{Obj}(\mathcal{I})$.

Prova. Pelo Teorema 2.27, $\widehat{B}$ é uma álgebra de Boole e

$$
\iota: \widehat{B} \longrightarrow B=\prod_{i \in I} B_{i}
$$

é uma Ba-imersão que identifica $\widehat{B}$ com uma subálgebra de $B$, estabelecendo (a). Para (b), seja $S \subseteq \widehat{B}$; uma vez que $B$ é completa (5.36.(c)) com estrutura produto, temos

$$
\bigvee \iota(S)=\left\langle\bigvee \pi_{i}(\iota(S))\right\rangle_{i \in I^{*}}
$$

Agora observe que, como as projeções coordenadas e os morfismos $f_{\alpha},(i \stackrel{\alpha}{\rightarrow} j)$ são $B a$-morfismos completos, temos

$f_{\alpha}\left(\bigvee \pi_{i}(\iota(S))\right)=\bigvee f_{\alpha}\left(\pi_{i}(\iota(S))\right)=\bigvee f_{\alpha}\left(f_{i}(S)\right)=\bigvee f_{j}(S)=\bigvee \pi_{j}(\iota(S))$

mostrando que $\bigvee \iota(S)$ está na imagem de $\iota$, isto é, existe $a \in \widehat{G}$ tal que $\iota(a)=\bigvee \iota(S)$. Está claro que com a ordem induzida por $B$ em $\widehat{B}, a=\bigvee S$ em $\widehat{B}$. Como os $f_{i}$ são (essencialmente) a restrição das projeções coordenadas a $\widehat{B}$, segue que são Ba-morfismos completos. ${ }^{26}$

Como toda álgebra de Boole finita é completa e todo Ba-morfismo entre álgebras finitas é completo, o resultado acima implica o

Corolário 5.38. Álgebras de Boole profinitas são completas.

5.39. Sejam $\langle I, \leq\rangle$ um conjunto parcialmente ordenado e dirigido e

$$
\mathcal{G}=\left\langle G_{i} ; f_{j i}\right\rangle
$$

um sistema cofiltrante de grupos finitos e reduzidos sobre $I$. Se aplicarmos o funtor envoltória Booleana a este sistema (3.48.(e)), determinamos um sistema cofiltrante de álgebras de Boole finitas e Ba-morfismos sobre I

$$
\mathcal{B}=\left\langle B_{G_{i}} ; B\left(f_{j i}\right)\right\rangle
$$

\footnotetext{
${ }^{26}$ Esta prova é válida, ipsis literis, na categoria dos reticulados e dos reticulados completos, com morfismos que preservam $\bigvee$ e $\bigwedge$.
} 
Em mais detalhe, se $i \leq j \leq k$ em $I$, temos diagramas comutativos de SG-morfismos e Bamorfismos
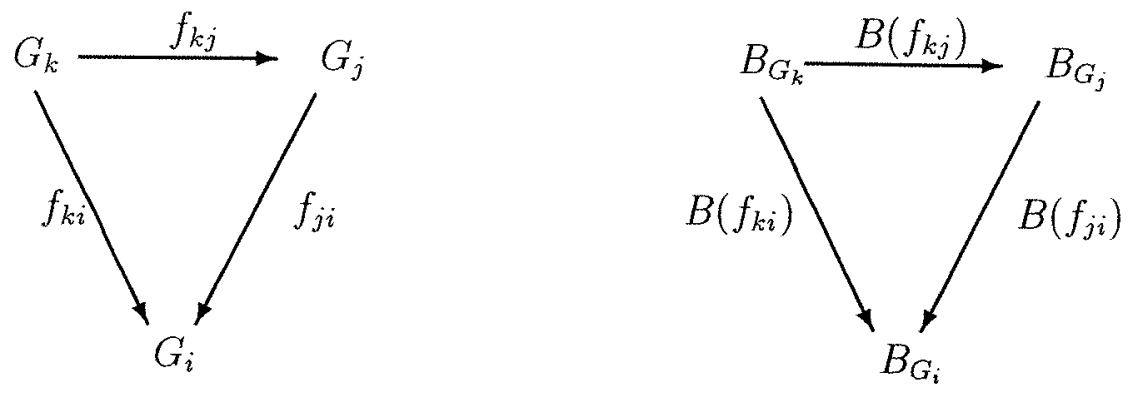

onde o segundo diagrama é obtido do primeiro pela aplicação do funtor envoltória Booleana do Teorema 3.48.(e), indicado por $B$. Assim, $\mathcal{B}$ é, de fato, um sistema cofiltrante na categoria $\mathbf{B a}$ sobre $I$. Uma vez que $B_{G_{i}}$ é a álgebra de abertos e fechados de $X_{G_{i}}$ (o espaço de ordens de $G_{i}$ ) e este é finito (pois $X_{G_{i}} \subseteq 2^{G_{i}}$ ), as álgebras booleanas $B_{G_{i}}$ são finitas. Assim $\mathcal{B}$ é um sistema cofiltrante de álgebras de Boole completas e Ba-morfismos completos.

Dos itens (a) e (c) no Teorema 3.48, sabemos que para $i \in I$, temos uma SG-imersão completa e natural ${ }^{27}$

$$
\varepsilon_{G_{i}}: G_{i} \longrightarrow B_{G_{i}}
$$

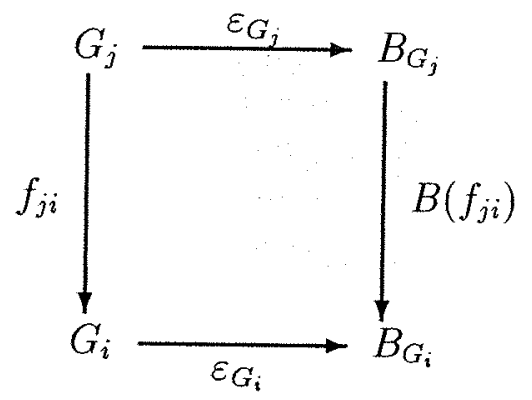

tal que o diagrama acima à direita é comutativo. Deste modo, a coleção $\varepsilon=\left\{\varepsilon_{G_{i}}: i \in I\right\}$ é um morfismo de sistemas cofiltrantes (2.29)

$$
\varepsilon: \mathcal{G} \longrightarrow \mathcal{B}
$$

tal que para todo $i \in I, \varepsilon_{G_{i}}$ é uma imersão completa. Sejam

$$
\left\langle\widehat{G}, g_{i}\right\rangle=\lim _{\leftarrow} \mathcal{G} \quad \text { e }\left\langle\widehat{B}, \beta_{i}\right\rangle=\lim \mathcal{B} .
$$

Sabemos de 5.11 que $\widehat{G}$ é um grupo especial reduzido e que a imersão canônica

$$
\iota: \widehat{G} \longrightarrow G=\prod_{i \in I} G_{i}
$$

preserva subformas, sendo portanto uma imersão completa (3.21.(b)). Por outro lado, 5.37 mostra que $\widehat{B}$ é uma álgebra de Boole completa, e portanto, por 3.47, um grupo especial reduzido; além disso, os Ba-morfismos $\beta_{i}$ são completos.

Pela Proposição 2.30, existe um único SG-morfismo

\footnotetext{
${ }^{27}$ Onde $B_{G_{i}}$ tem a estrutura de grupo especial reduzido descrita em 3.4.3.
} 
$(\varepsilon)$

$$
h=\underline{\lim \varepsilon}: \lim \mathcal{G} \longrightarrow \lim \mathcal{B}
$$

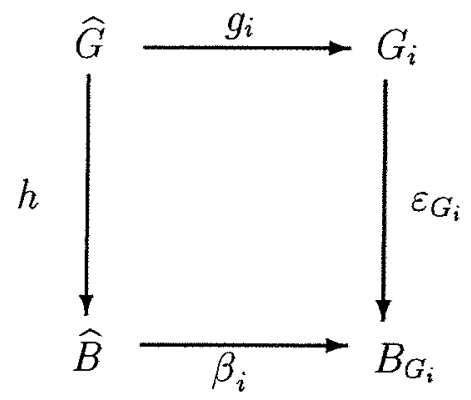

que faz comutar o quadrado acima.

Proposição 5.40. Com a notação em 5.39,

a) OSG-morfismo $h: \widehat{G} \longrightarrow \widehat{B}$ é uma imersão completa.

b) A envoltória Booleana de $\widehat{G}, B_{\hat{G}}$, é (isomorfa) a subálgebra de Boole de $\widehat{B}$ gerada por $h(\widehat{G})$.

\section{Prova.}

a) $\operatorname{Sejam} \varphi, \psi$ formas de mesma dimensão sobre $\widehat{G}$, tais que $h \star \varphi \equiv_{\hat{B}} h \star \psi$. Para $i \in I$, essa relação implica.

$$
\left(\beta_{i} \circ h\right) \star \varphi \equiv_{B_{G_{i}}}\left(\beta_{i} \circ h\right) \star \psi,
$$

e assim, a comutatividade do diagrama $(\varepsilon)$ em 5.39 acarreta

$$
\left(\varepsilon_{G_{i}} \circ g_{i}\right) \star \varphi \equiv_{B_{G_{i}}}\left(\varepsilon_{G_{i}} \circ g_{i}\right) \star \psi .
$$

Como $\varepsilon_{G_{i}}$ é uma imersão completa (3.48.(a)), a isometria precedente fornece

$$
g_{i} \star \varphi \equiv_{G_{i}} g_{i} \star \psi .
$$

Observe que (I) vale para todo $i \in I$; como $g_{i}=\pi_{i} \circ \iota{ }^{28}$ e a isometria em $G$ é dada coordenada a coordenada, concluímos que

$$
\iota \star \varphi \equiv_{G} \iota \star \psi \text {. }
$$

Uma vez que $\iota$ é uma imersão completa, obtemos $\varphi \equiv_{\hat{G}} \psi$, completando a prova de (a).

b) Seja $\varepsilon_{\hat{G}}: \widehat{G} \longrightarrow B_{\hat{G}}$ a envoltória Booleana de $\widehat{G}$. Como $h: \widehat{G} \longrightarrow \widehat{B}$ é uma imersão completa, o item (3) do Teorema 5.2 em [DM1] garante que $B(h)$ é um Ba-morfismo injetor. Por outro lado a propriedade universal da envoltória Booleana no item (4) do Teorema 4.17 em [DM1] mostra que o seguinte diagrama é comutativo :

\footnotetext{
${ }^{28} g_{i}=\pi_{i} \circ \ell ;$ veja diagrama (D) na prova do Teorema 5.11, página 133.
} 


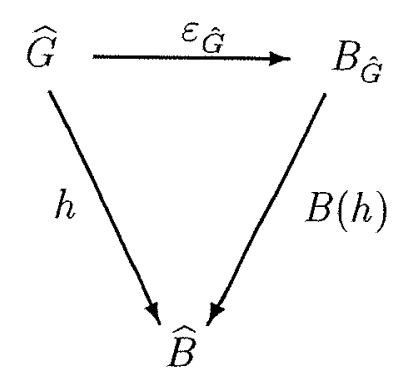

Por 3.48.(b), para todo $u \in B_{\hat{G}}$, existe uma família $\left\{F_{1}, \ldots, F_{n}\right\}$ de subconjuntos finitos de $\widehat{G}$ tal que

$$
u=\bigcup_{k=1}^{n} \bigcap_{a \in F_{k}} \varepsilon_{\hat{G}}(a)
$$

Como (II) é um diagrama comutativo de morfismos injetores e a imagem de $B_{\hat{G}}$ por $B(h)$ é uma subálgebra de $\widehat{B}$ que lhe é isomorfa, segue imediatamente de (III) que $B_{\hat{G}}$ é isomorfa à subalgebra de Boole de $\widehat{B}$ gerada por $h(\widehat{G})$, encerrando a prova.

\subsection{A Envoltória Profinita}

A notação introduzida no Capítulo 4 será de uso constante daqui em diante.

5.41. Construção. Seja $G$ um grupo especial reduzido. Seja

$$
\mathcal{F}(G)=\{\Delta \in \operatorname{Ssat}(G): \Delta \text { tem índice finito em } G\} .
$$

Por 4.15.(c), $\mathcal{F}(G)$ é um filtro em $\operatorname{Ssat}(G)$ e portanto um conjunto dirigido para baixo pela inclusão.

Para $\Gamma \subseteq \Delta \subseteq \Sigma$ em $\mathcal{F}(G)$, por 4.6 temos diagramas comutativos de SG-morfismos
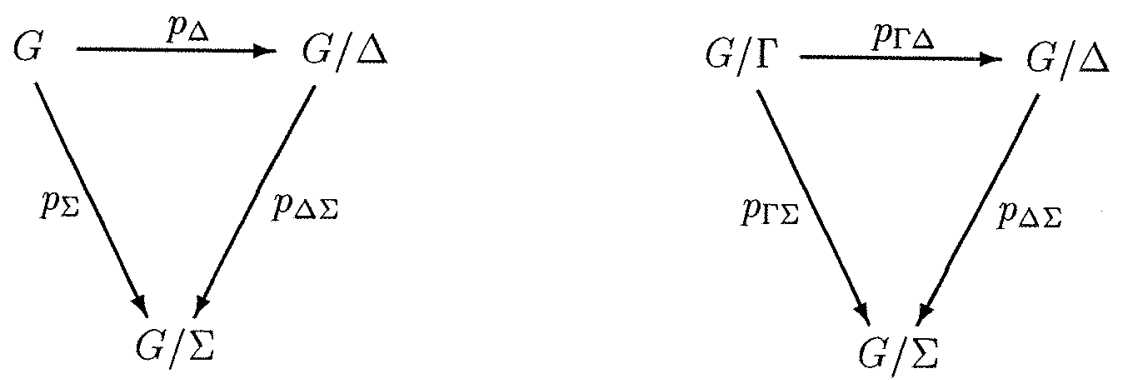

que mostram que

(I) $\mathcal{G}=\left\langle G / \Delta ; p_{\Delta \Sigma}\right\rangle$ é um sistema cofiltrante de grupos especiais reduzidos, finitos e discretos sobre $\mathcal{F}(G)=\left(\mathcal{F}(G)^{o p}\right)^{o p}$

(II) $\left\langle G ; p_{\Delta}\right\rangle$ é um cone sobre o sistema cofiltrante $\mathcal{G}$.

Sejam 
(III) $\left\langle\mathcal{P}(G) ; \mathfrak{p}_{\Delta}\right\rangle=\lim \mathcal{G}$; pelo Teorema $5.11, \mathcal{P}(G)$ é um grupo especial reduzido e Booleano 29 . Indicaremos um elemento típico de $\mathcal{P}(G)$ por $a=\left\langle a_{\Delta}\right\rangle_{\Delta \in \mathcal{F}(G)}$.

(IV) Os SG-morfismos $\mathfrak{p}_{\Delta}: \mathcal{P}(G) \longrightarrow G / \Delta$ são contínuos e o conjunto

$$
T(G)=\left\{\operatorname{ker} \mathfrak{p}_{\Delta}: \Delta \in \mathcal{F}(G)\right\}
$$

é um pf-sistema em $\mathcal{P}(G)$.

(V) Existe um único SG-morfismo, $\eta_{G}: G \longrightarrow \mathcal{P}(G)$, tal que para todo $\Delta \in \mathcal{F}(G)$ o seguinte diagrama é comutativo :

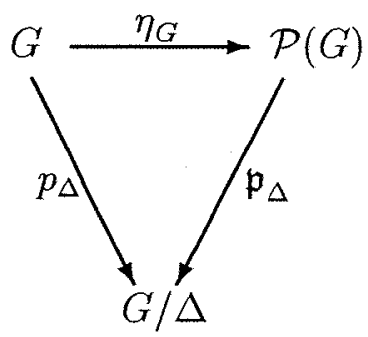

Observe que a notação é consistente com a de 5.23 , pois $\mathfrak{p}_{\Delta}$ é necessariamente sobrejetor. É possível dar uma expressão explícita para o SG-morfismo $\eta_{G}$ :

$$
\text { Para } u \in G, \eta_{G}(u)=\langle u / \Delta\rangle_{\Delta \in \mathcal{F}(G)}=\left\langle p_{\Delta}(u)\right\rangle_{\Delta \in \mathcal{F}(G)} .
$$

(VI) Como no Teorema 5.11, seja.

$$
\iota: \mathcal{P}(G) \longrightarrow \prod_{\Delta \in \mathcal{F}(G)} G / \Delta
$$

o SG-morfismo que reflete subformas e identifica o limite projetivo com um subgrupo especial do produto dos quocientes reduzidos e finitos de $G$. Como no caso geral, os morfismos $\mathfrak{p}_{\Delta}$ são a restrição à $\mathcal{P}$ das projeções coordenadas do produto, isto é, $\mathfrak{p}_{\Delta}=\pi_{\Delta} \circ \ell$.

Definição 5.42. O grupo especial Booleano reduzido construído em 5.41 denomina-se envoltória profinita de $G$ e o $S G$-morfismo $\eta_{G}$ é a imersão canônica de $G$ em $\mathcal{P}(G)$.

As propriedades fundamentais desta construção constam do

Teorema 5.43. Seja $G$ um grupo especial reduzido. Com a notação acima,

a) $\eta_{G}$ é um $S G$-monomorfismo cuja imagem é densa em $\mathcal{P}(G)$ e que reflete subformas.

b) Se $H$ é um grupo especial Booleano reduzido e $f: G \longrightarrow H$ é um SG-morfismo, existe um único $S G$-morfismo contínuo, $\tilde{f}: \mathcal{P}(G) \longrightarrow H$, que faz comutar o diagrama abaixo a esquerda:

${ }^{29}$ Utilizaremos esta expressão como sinônimo de grupo especial reduzido profinito: veja a caracterização 5.17. 

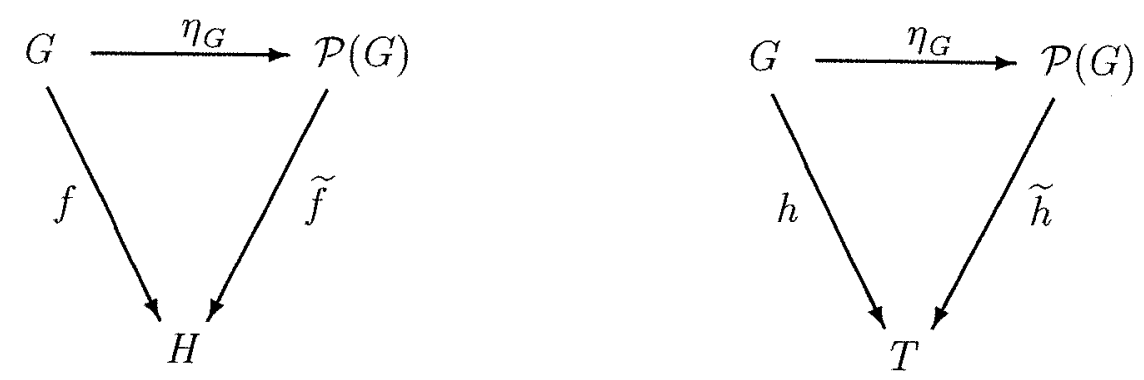

c) Sejam $T$ um grupo especial Booleano reduzido e $h: G \longrightarrow T$ um SG-morfismo injetor que satisfazem as seguintes condiçôes:

(1) $\eta_{G}: G \longrightarrow \mathcal{P}(G)$ é contínua na topologia induzida por $T$ em $G$ via $h$, isto é, se $\Delta \in \mathcal{F}(G)$, existe $W$ subgrupo saturado, aberto e fechado, de indice finito em $T$, tal que $\eta_{G}\left(h^{-1}(W)\right) \subseteq$ ker $\mathfrak{p}_{\Delta}$.

(2) $h^{4}\left(\equiv_{G}\right)$ é denso em $\equiv_{T}$ na topologia produto, isto é, se $t_{1}, t_{2}, t_{3}, t_{4} \in T$ e $W$ é um subgrupo aberto, saturado e de indice finito em $T$,

$$
\left\langle t_{1}, t_{2}\right\rangle \equiv_{T}\left\langle t_{3}, t_{4}\right\rangle \Rightarrow\left\{\begin{array}{l}
\exists u_{1}, u_{2}, u_{3}, u_{4} \in G \text { tais que } \\
\left\langle u_{1}, u_{2}\right\rangle \equiv_{G}\left\langle u_{3}, u_{4}\right\rangle \quad e \\
h\left(u_{i}\right) \cdot t_{i} \in W, 1 \leq i \leq 4
\end{array}\right.
$$

Então; com a notação em (b), $\widetilde{h}: \mathcal{P}(G) \longrightarrow T$ é o único SG-isomorfismo contínuo que faz comutar o diagrama acima a direita. Em particular, $\tilde{h}$ é um homeomorfismo e o SG-morfismo $h$ reflete subformas.

Prova. Seja $\iota: \mathcal{P}(G) \longrightarrow \prod_{\Delta \in \mathcal{F}(G)} G / \Delta$ a SG-imersão que reflete subformas, conforme o Teorema 5.11.

a) É imediato que $\eta_{G}$ é um SG-morfismo. Como $G$ é reduzido, segue do Teorema de separação (3.30.(c)) que

$$
\{1\}=\bigcap\{\Sigma \in \mathcal{F}(G): \Sigma \text { tem índice } 2 \text { em } G\} .
$$

Se $\eta_{G}(x)=1$, então $\iota\left(\eta_{G}(x)\right)=1$ e portanto $x / \Sigma=1$ para todo saturado maximal em $G$. Assim, (I) implica que $\eta_{G}$ é injetora. Uma vez que

$$
T=\left\{\mathfrak{p}_{\Delta}: \Delta \in \mathcal{F}(G)\right\}
$$

é um sistema fundamental de vizinhanças abertas da unidade do grupo topológico $\mathcal{P}(G)$, para verificar a densidade da imagem de $\eta_{G}{ }^{30}$ é suficiente mostrar que

$$
\forall a \in \mathcal{P}(G) \text { e } \forall \Delta \in \mathcal{F}(G) \text {, existe } u \in G \text { tal que } \eta_{G}(u) \cdot a \in \operatorname{ker} \mathfrak{p}_{\Delta} \text {. }
$$

Fixados $a \in \mathcal{P}(G)$ e $\Delta \in \mathcal{F}(G)$, como o diagrama

\footnotetext{
${ }^{30}$ Veja também 5.6.
} 
(III)

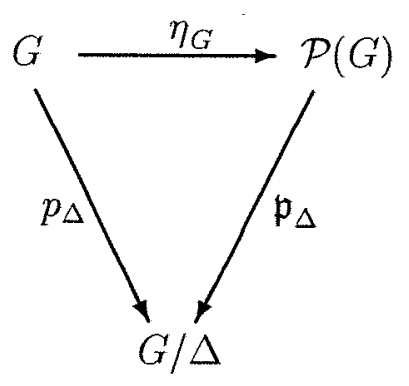

é comutativo, existe $u \in G$ tal que $\mathfrak{p}_{\Delta}(a)=a_{\Delta}=p_{\Delta}(u)=\mathfrak{p}_{\Delta}\left(\eta_{G}(u)\right)$. Mas então,

$$
\mathfrak{p}_{\Delta}\left(\eta_{G}(u) \cdot a\right)=\mathfrak{p}_{\Delta}\left(\eta_{G}(u)\right) \cdot \mathfrak{p}_{\Delta}(a)=a_{\Delta} \cdot a_{\Delta}=1,
$$

estabelecendo (II), como desejado. A prova que $\eta_{G}$ reflete subformas é consequência do seguinte

\section{Fato 5.44.}

a) $S e \varphi\left(v_{1}, \ldots, v_{n}\right)$ é uma fórmula $p p$ em $L_{S G}$ e $\bar{g} \in G^{n}$ é tal que $G \models \neg \varphi[\bar{g}]$, então o conjunto

$$
S(G, \bar{g}, \varphi)=\{\Delta \in S \operatorname{sat}(G): G / \Delta \vDash \neg \varphi[\bar{g} / \Delta]\},
$$

parcialmente ordenado por inclusão, admite elemento maximal.

b) $\eta_{G}$ reflete índice de Witt.

Prova. O item (a) está provado em [Ast2] e em [Mar7], na linguagem dual dos espaços de ordem abstratos (com os devidos créditos); assim omitiremos sua prova ${ }^{31}$.

b) Seja $\varphi$ uma forma sobre $G$, tal que $k=\operatorname{ind}_{W}\left(\eta_{G} \star \varphi\right)$ em $\mathcal{P}(G)$. Uma vez que $\iota$ reflete subformas (5.11.(b)), obtemos

$$
\left(\iota \quad \circ \eta_{G}\right) \star \varphi \text { tem índice de Pfister } k \text { em } \prod_{\Delta \in \mathcal{F}(G)} G / \Delta \text {. }
$$

Uma vez que a isometria no produto definida é coordenada à coordenada e que

$$
\pi_{\Delta} \circ \iota \circ \eta_{G}=\mathfrak{p}_{\Delta} \circ \eta_{G}=p_{\Delta},
$$

concluímos que

$$
\forall \Delta \in \mathcal{F}(G), \quad G / \Delta \models \operatorname{ind}_{W}\left(p_{\Delta} \star \varphi\right) \geq k .
$$

Suponha por absurdo que $\operatorname{ind}_{W}(\varphi)<k$ em $G$. Como a frase

$$
\text { "ind }{ }_{W}(\varphi) \geq k "
$$

é uma fórmula pp tendo como paramêtros os coeficientes de $\varphi$, o item (a) garante que existe um subgrupo saturado $\Sigma$ em $G$ tal que

$$
G / \Sigma \models \operatorname{ind}_{W}\left(p_{\Sigma} \star \varphi\right)<k \quad \text { e } \quad \forall \Delta \text { tal que } \Sigma \subsetneq \Delta, G / \Delta \models \operatorname{ind}_{W}\left(p_{\Delta} \star \varphi\right) \geq k .
$$

Pela Proposição 3.53, (V) implica que $G / \Sigma$ tem comprimento de cadeia finito. Assim, o Corolário 3.52 fornece um subgrupo saturado $\Gamma$ de índice finito em $G / \Sigma$ tal que

$$
\operatorname{Em}(G / \Sigma) / \Gamma, \operatorname{ind}_{W}\left(p_{\Gamma} \star \varphi\right)<k .
$$

\footnotetext{
${ }^{31}$ Que é essencialmente uma aplicação do Lema de Zorn.
} 
Seja $\Delta=p_{\Sigma}^{-1}(\Gamma)$; então $\Delta$ é um subgrupo saturado de índice finito em $G$, tal que o seguinte diagrama é comutativo

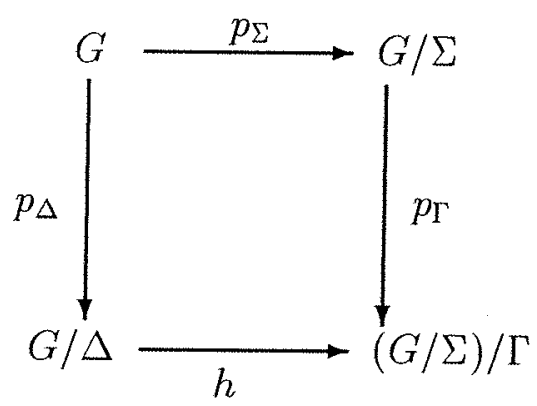

onde $h$ é um SG-isomorfismo. ${ }^{32}$

Segue de (VI) que $G / \Delta \vDash$ ind $_{W}\left(p_{\Delta} \star \varphi\right)<k$, contradizendo (IV) e encerrando a prova do Fato.

Do item (b) em 5.44 e da Proposição 3.21.(c).(2) concluímos que $\eta_{G}$ reflete subformas, completando a prova do item (a) do Teorema.

b) Pelo item (a), $\eta_{G}(G)$ é um subgrupo denso de $\mathcal{P}(G)$. Se $\Delta \in \mathcal{F}(G)$, a comutatividade do diagrama (III) (página 156) implica que

$$
\Delta=\eta_{G}^{-1}\left(\operatorname{ker} \mathfrak{p}_{\Delta}\right) \text {. }
$$

Assim,

$$
\forall \Delta \in \mathcal{F}(G), \quad \eta_{G}(\Delta)=\eta_{G}(G) \cap \operatorname{ker} \mathfrak{p}_{\Delta} .
$$

Podemos considerar $f$ como um SG-morfismo de $\eta_{G}(G)$ em $H^{33}$. Vamos mostrar que $f$ é contínuo na topologia induzida por $\mathcal{P}(G)$ em $\eta_{G}(G)$; para isto é suficiente verificar sua continuidade em 1 (5.2.(c)). Como $H$ é um grupo especial Booleano, seja $\mathcal{V}$ um pf-sistema em $H$. Se $U \in \mathcal{V}$, o fato que $f$ é um SG-morfismo implica que $\Delta=f^{-1}(U)$ é um subgrupo saturado de índice finito em $G$. Uma vez que

$$
f\left(\eta_{G}(\Delta)\right) \subseteq U \quad \text { e } \quad\left\{\operatorname{ker} \mathfrak{p}_{\Delta}: \Delta \in \mathcal{F}(G)\right\} \text { é um pf-sistema em } \mathcal{P}(G),
$$

(VII) implica que $f$ é contínuo no ponto 1 na topologia induzida por $\mathcal{P}(G)$, como desejado. Segue do Teorema 5.7.(a) que $f$ é uniformemente contínua. Como $H$ é um grupo topológico compacto Hausdorff, 5.7.(d) garante a existência de um único morfismo contínuo de grupos,

$$
\widetilde{f}: \mathcal{P}(G) \longrightarrow H
$$

que torna comutativo o diagrama do enunciado. Resta mostrar que $\widetilde{f}$ é um SG-morfismo. Como $-1 \in \eta_{G}(G)$ e $f(-1)=-1$, temos que $\widetilde{f}(-1)=-1$. Para verificar que $\widetilde{f}$ preserva isometria binária, sejam $a_{1}, a_{2}, a_{3}, a_{4}$ elementos de $\mathcal{P}(G)$ tais que

$$
\left\langle a_{1}, a_{2}\right\rangle \equiv \mathcal{P}(G)\left\langle a_{3}, a_{4}\right\rangle \text {. }
$$

Como $\equiv_{H}$ é fechada em $H^{4}(5.17)$, para provar que

\footnotetext{
${ }^{32}$ Veja 4.13.(b)

${ }^{33}$ Pelo item (a), $\eta_{G}$ é um isomorfimo de grupos especiais entre $G$ e $\eta_{G}(G)$.
} 


$$
\left\langle\widetilde{f}\left(a_{1}\right), \widetilde{f}\left(a_{2}\right)\right\rangle \equiv_{H}\left\langle\widetilde{f}\left(a_{3}\right), \widetilde{f}\left(a_{4}\right)\right\rangle
$$

é suficiente verificar que para todo aberto $W$ de $H^{4}$, contendo $\left\langle\widetilde{f}\left(a_{1}\right), \widetilde{f}\left(a_{2}\right), \widetilde{f}\left(a_{3}\right), \widetilde{f}\left(a_{4}\right)\right\rangle$, temos $W \cap \equiv_{H} \neq \emptyset$. Como $\widetilde{f}$ é contínua e

$$
T=\left\{\operatorname{ker} \mathfrak{p}_{\Delta}: \Delta \in \mathcal{F}(G)\right\}
$$

é um pf-sistema em $\mathcal{P}(G)$, existe $\Delta \in \mathcal{F}(G)$ tal que a vizinhança aberta e fechada de $\left\langle a_{1}, a_{2}, a_{3}, a_{4}\right\rangle$ em $\mathcal{P}(G)^{4}$ dada por

$$
V=a_{1} \operatorname{ker} \mathfrak{p}_{\Delta} \times a_{2} \operatorname{ker} \mathfrak{p}_{\Delta} \times a_{3} \operatorname{ker} \mathfrak{p}_{\Delta} \times a_{4} \operatorname{ker} \mathfrak{p}_{\Delta}
$$

$\operatorname{satisfaz}(\widetilde{f})^{4}(V) \subseteq W$

Como $\mathfrak{p}_{\Delta}: \mathcal{P}(G) \longrightarrow G / \Delta$ é um SG-morfismo, a relação (VIII) acarreta

$$
\left\langle a_{1 \Delta}, a_{2 \Delta}\right\rangle \equiv G / \Delta\left\langle a_{3 \Delta}, a_{4 \Delta}\right\rangle .
$$

Uma vez que o diagrama em (III) acima (página 156) é comutativo, a relação (IX) e o Teorema 3.29.(a) fornecem $u_{1}, u_{2}, u_{3}, u_{4} \in G$ tais que

$$
(\mathrm{X})\left\langle u_{1}, u_{2}\right\rangle \equiv_{G}\left\langle u_{3}, u_{4}\right\rangle ; \quad(\mathrm{XI}) p_{\Delta}\left(u_{i}\right)=\mathfrak{p}_{\Delta}\left(\eta_{G}\left(u_{i}\right)\right)=a_{i \Delta}, \quad 1 \leq i \leq 4 .
$$

Segue de (XI) que $a_{i} \cdot \eta_{G}\left(u_{i}\right) \in \operatorname{ker} \mathfrak{p}_{\Delta}$ e portanto

$$
\left\langle\eta_{G}\left(u_{1}\right), \eta_{G}\left(u_{2}\right), \eta_{G}\left(u_{3}\right), \eta_{G}\left(u_{4}\right)\right\rangle \in V
$$

Observe que acabamos de provar o seguinte

Fato 5.45. A imagem de $\equiv_{G}$ por $\eta_{G}^{4}$ é densa em $\equiv_{\mathcal{P}(G)}$ na topologia produto em $\mathcal{P}(G)^{4}$.

Como $\tilde{f}\left(\eta_{G}\left(u_{i}\right)\right)=f\left(u_{i}\right), \quad 1 \leq i \leq 4$, e $f$ é um SG-morfismo, a relação $(\mathrm{X})$ implica $\left\langle\widetilde{f}\left(\eta_{G}\left(u_{1}\right)\right), \tilde{f}\left(\eta_{G}\left(u_{2}\right)\right)\right\rangle=\left\langle f\left(u_{1}\right), f\left(u_{2}\right)\right\rangle \equiv_{H}\left\langle f\left(u_{3}\right), f\left(u_{4}\right)\right\rangle=\left\langle\tilde{f}\left(\eta_{G}\left(u_{3}\right)\right), \tilde{f}\left(\eta_{G}\left(u_{4}\right)\right)\right\rangle$ agora, (XII) e o fato que $(\widetilde{f})^{4}(V) \subseteq W$ fornecem $W \cap \equiv_{H} \neq \emptyset$, estabelecendo (b).

c) Os itens (1) e (2) são a axiomatização das propriedades necessárias para provar o item (b). Uma análise da prova mostra que o item (2) foi fundamental - veja 5.45 na prova de (b) -, enquanto que, naquele caso, o item (1) vinha de graça, pois qualquer SG-morfismo de $G$ em um grupo especial Booleano e reduzido é contínuo na topologia induzida por $\mathcal{P}(G)$ em $G$ via $\eta_{G}$. No caso geral, é necessário assumir também esta condição, que não será verdade em geral. Note que (2) implica que $h(G)$ é denso em $T$.

Assim, o mesmo método utilizado na prova de (b) mostra que existe um único SG-morfismo contínuo, $g: T \longrightarrow \mathcal{P}(G){ }^{34}$, que faz comutar o primeiro diagrama abaixo a direita, enquanto que (b) garante que existe um único $\widetilde{h}$ que faz comutar o diagrama do meio:

\footnotetext{
${ }^{34} \mathrm{~A}$ extensão de $\eta_{G}: h(G) \longrightarrow \mathcal{P}(G)$.
} 

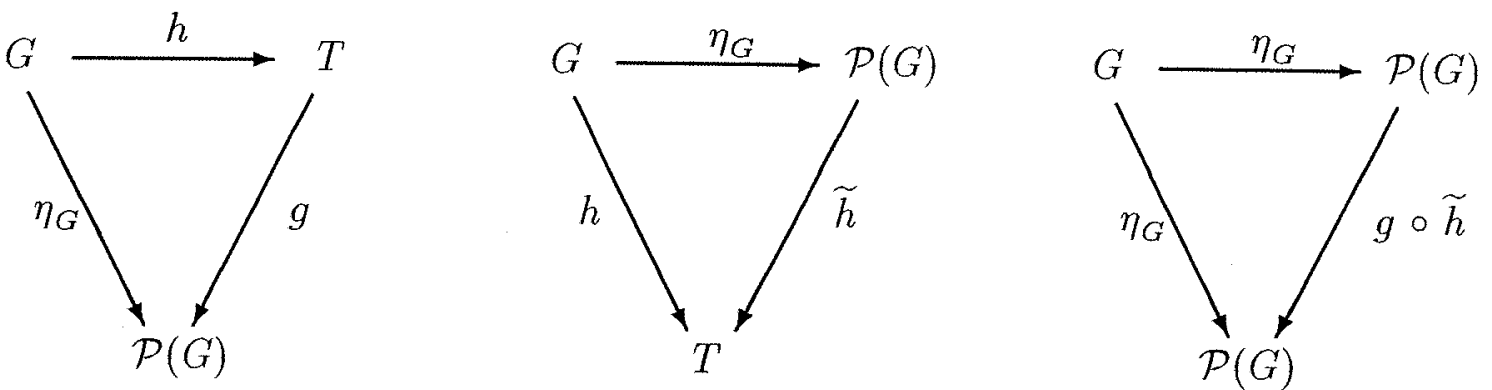

É imediato que a composição $g \circ \widetilde{h}$ é um SG-morfismo contínuo que faz comutar o último diagrama acima. A unicidade em (b) implica então que $g \circ \widetilde{h}=I d_{\mathcal{P}(G)}$. Segue de 2.45.(a).(1) que $\widetilde{h}$ é um SG-morfismo contínuo puro e portanto uma imersão completa e contínua (3.21.(b)). Como $\mathcal{P}(G)$ é um espaço compacto e $T$ é Hausdorff, $\widetilde{h}(\mathcal{P}(G))$ é fechado em $T$. O diagrama do meio acima garante que $h(G) \subseteq \widetilde{h}(\mathcal{P}(G))$; uma vez que $h(G)$ é denso em $T$, concluímos que $\widetilde{h}$ é sobrejetor. Assim, $\widetilde{h}$ é uma imersão completa e sobrejetora, constituindo-se portanto em um SG-isomorfismo, como desejado. As demais afirmações no enunciado estão claras, encerrando a prova.

Observação 5.46. Seja $\eta_{G}: G \longrightarrow \mathcal{P}(G)$ a envoltória profinita de $G$.

a) Se $G$ é um grupo finito, reduzido e discreto, então $\{1\}$ é de índice finito em $G$ e é o menor elemento de $\mathcal{F}(G)$. Logo $\mathcal{P}(G)$ é finito e discreto e $\eta_{G}: G \longrightarrow \mathcal{P}(G)$ é isomorfismo de grupos especiais topológicos.

b) Como já observado na prova do Teorema 5.43, segue do diagrama (III) na prova do seu item (a) (página 156) ${ }^{35}$, que para todo $\Delta \in \mathcal{F}(G)$

$$
\Delta=\eta_{G}^{-1}\left(\operatorname{ker} \mathfrak{p}_{\Delta}\right)
$$

Uma vez que

$$
T=\left\{\operatorname{ker} \mathfrak{p}_{\Delta}: \Delta \in \mathcal{F}(G)\right\}
$$

é um pf-sistema em $\mathcal{P}(G)$, a topologia de grupo topológico Hausdorff induzida em $G$ por $\mathcal{P}(G)$ via $\eta_{G}$ tem como sistema fundamental de vizinhaças de 1 exatamente $\mathcal{F}(G)$.

c) Seja $h: G \longrightarrow T$ um SG-morfismo injetor, onde $T$ é um grupo especial Booleano reduzido. Seja $\mathcal{V}$ um pf-sistema em $T$. Tendo em conta o item (a) e o fato que $\eta_{G}$ é injetora ${ }^{36}$, a condição (1) em 5.43.(c) pode ser reescrita como

$\left(1^{*}\right)$ Para todo $\Delta \in \mathcal{F}(G)$, existe $W \in \mathcal{V}$ tal que $h^{-1}(W) \subseteq \Delta$.

Esta formulação será útil nas aplicações que faremos de 5.43.(c).

d) Segue de 5.43.(c) que, nas condições do enunciado, as topologias induzidas em $G$ por $\mathcal{P}(G)$ via $\eta_{G}$ e por $T$ via $h$ são as mesmas.

\footnotetext{
${ }^{35} \mathrm{O}$ mesmo que aparece em 5.41.(V).

${ }^{36}$ E portanto $\eta_{G}^{-1}\left(\eta_{G}(A)\right)=A, \forall A \subseteq G$.
} 
Aqui estão algumas primeiras consequências interessantes do Teorema 5.43 :

Corolário 5.47. Seja $G$ um grupo especial reduzido e $\Sigma$ um subgrupo saturado de $G$.

a) Para todo $\sigma \in X_{G}$, existe um único $\widetilde{\sigma} \in X_{\mathcal{P}(G)}$ tal que $\widetilde{\sigma}$ é contínuo $e \quad \sigma=\widetilde{\sigma}$ o $\eta_{G}$.

b) Se $\mathcal{M}(\Sigma)=\left\{\right.$ ker $\sigma: \sigma \in X_{G}$ e $\left.\Sigma \subseteq \operatorname{ker} \sigma\right\},{ }^{37}$ definimos

$$
\bar{\Sigma}=\bigcap\{\operatorname{ker} \tilde{\sigma}: \sigma \in \mathcal{M}(\Sigma)\}
$$

Então, $\bar{\Sigma}$ é um subgrupo saturado e fechado de $\mathcal{P}(G)$ e o quociente $\mathcal{P}(G) \bar{\Sigma}$ é um grupo especial Booleano reduzido.

Prova. O item (a) segue imediatamente de 5.43.(b), pois $\mathbb{Z}_{2}$ com a topologia discreta é um grupo especial Booleano reduzido. Para (b), como $\widetilde{\sigma}$ é contínuo, para cada $\sigma \in \mathcal{M}(\Sigma)$, está claro que $\bar{\Sigma}$ é fechado e saturado. O fato que $\mathcal{P}(G) / \bar{\Sigma}$ é um grupo especial Booleano reduzido é consequência imediata do Teorema 5.28.

Corolário 5.48. Se G é um grupo especial reduzido então existe um corpo Pitagórico $F$ e um $S G$-morfismo, $j: G \longrightarrow \mathcal{G}(F)$, que reflete subformas.

Prova. Como $\mathcal{P}(G)$ é um grupo especial profinito, segue do Teorema 5.8.(b) que existe um corpo Pitagórico $F$ e um SG-morfismo puro, $f: \mathcal{P}(G) \longrightarrow \mathcal{G}(F)$. Uma vez que $\eta_{G}: G \longrightarrow$ $\mathcal{P}(G)$ reflete subformas (5.43.(a)), a composição $f \circ \eta_{G}: G \longrightarrow \mathcal{G}(F)$ também reflete subformas.

Além do interesse em comparar grupos especiais com o de corpos, o Corolário 5.48 é útil para fornecer uma prova diferente daquela em [Ast2] da generalização para grupos reduzidos do critério de Pfister para subformas :

Corolário 5.49. (O Critério de Pfister para subformas) Sejam $G$ um grupo especial reduzido e $\varphi, \psi$ formas sobre $G$, com $\psi$ anisotrópica. As segintes condições são equivalentes :

(1) $\varphi \preceq_{G} \psi$;

(2) Para todo grupo especial $H$ e todo $S G$-morfismo $f: G \longrightarrow H$, $D_{H}(f \star \varphi) \subseteq D_{H}(f \star \psi)$.

Prova. O Lema 3.17.(c) garante que (1) $\Rightarrow(2)$. Para (2) $\Rightarrow(1)$, o Corolário 5.48 fornece um corpo Pitagórico, $F$, e um SG-morfismo que reflete subformas, $j: G \longrightarrow \mathcal{G}(F)$. A conclusão agora segue da versão para corpos do critério de Pfister enunciado no Teorema 3.45.

A definição de fan aparece em 3.41. Do Corolário 5.9 obtemos

\footnotetext{
${ }^{37}$ Portanto $\mathcal{M}(\Sigma)=X_{\Sigma}(G)($ veja $4.9 .(\mathrm{d})$ e 4.10 .(c)) .
} 
Corolário 5.50. Se $G$ é um grupo especial reduzido, então $G$ é um fan sse $\mathcal{P}(G)$ é um fan.

Prova. Se $\mathcal{P}(G)$ é um fan, o mesmo é verdade acerca de $G$, pois $\eta_{G}$ é um SG-morfismo injetor. Reciprocamente, como todo quociente de um fan é um fan, $\mathcal{P}(G)$ é um limite projetivo sobre o conjunto dirigido $\mathcal{F}(G)^{o p}$ de um sistema de fans finitos. Por $5.9, \mathcal{P}(G)$ é um fan.

\subsection{A Envoltória Profinita como Funtor}

Como já anunciado anteriormente, a notação do Capítulo 4 está em pleno vigor. Além disso, a notação em 5.41 será de uso constante.

5.51. Seja $f: G \longrightarrow H$ um SG-morfismo de grupos especiais reduzidos. Então, $f$ induz um morfismo de troca de base

$$
\alpha_{f}: \mathcal{G} \longrightarrow \mathcal{H}
$$

do sistema cofiltrante $\mathcal{G}$ sobre $\mathcal{F}(G)$ associado a $G$ para o sistema cofiltrante $\mathcal{H}$ sobre $\mathcal{F}(H)$ associado a $H$, da seguinte forma :

* Para $\Delta^{\prime} \in \mathcal{F}(H), \alpha_{f}\left(\Delta^{\prime}\right)=f^{*}\left(\Delta^{\prime}\right)$. A Proposição 4.2.(a) e o Lema 4.11.(c) garantem que , $\alpha_{f}$ é uma função bem definida e crescente de $\mathcal{F}(H)$ em $\mathcal{F}(G)$;

* Se $\Delta^{\prime}$ em $\mathcal{F}(H)$, seja $\Delta=f^{*}\left(\Delta^{\prime}\right)$; por 4.4 temos um SG-morfismo

$$
f_{\Delta^{\prime}}: G / \Delta \longrightarrow H / \Delta^{\prime}
$$

o morfismo derivado de $f$. Se $\Delta^{\prime} \subseteq \Sigma^{\prime}$ em $\mathcal{F}(H)$, a Proposição 4.6.(c) garante que o seguinte diagrama é comutativo, onde $\Delta=f^{*}\left(\Delta^{\prime}\right)$ e $\Sigma=f^{*}\left(\Sigma^{\prime}\right)$ :

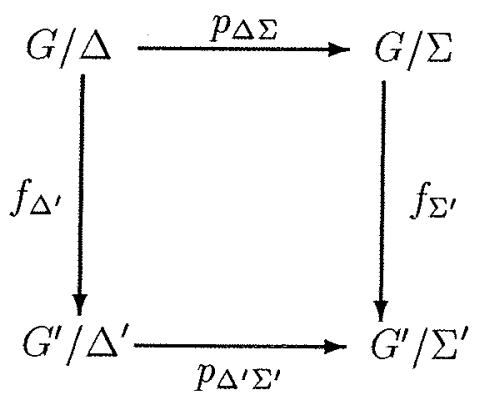

Tendo em vista 5.33, temos que $\alpha_{f}$ é de fato um morfismo de troca de base, como desejado.

Além disso:

* Se $h: H \longrightarrow K$ é um SG-morfismo de grupos especiais reduzidos, 4.2.(b).(2) e 4.6.(b) garantem que

(comp)

$$
\alpha_{h} \circ \alpha_{f}=\alpha_{h \circ f}
$$

* Para o RSG-morfismo identidade $I d_{H}: H \longrightarrow H, 4.2 .(\mathrm{b}) .(3)$ e 4.6.(a) garantem que 
(ident)

$$
\alpha_{I d_{H}}=I d_{\mathcal{H}}
$$

Com os preliminares em 5.51, a Proposição 5.34 fornece

Teorema 5.52. A envoltória profinita fornece um funtor

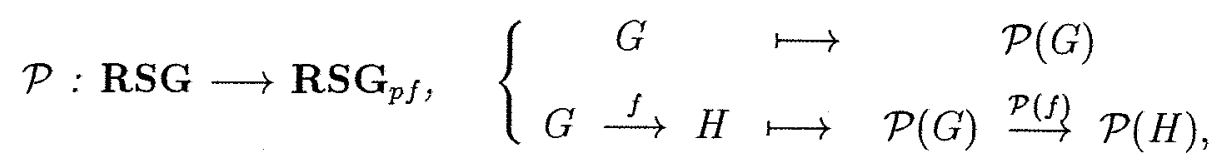

tal que para todo $S G$-morfismo $f: G \longrightarrow H$ de grupos especiais reduzidos o seguinte diagrama é comutativo:

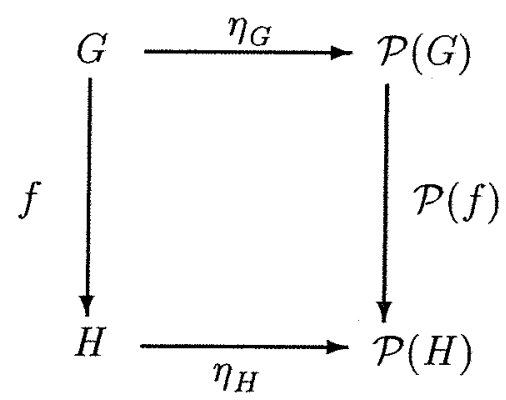

O SG-morfismo contínuo $\mathcal{P}(f)$ tem a seguinte descrição explícita : se $a=\left\langle a_{\Sigma}\right\rangle_{\Sigma \in \mathcal{F}(G)} \in \mathcal{P}(G)$ então

$$
\mathcal{P}(f)(a)=\left\langle b_{\Delta^{\prime}}\right\rangle_{\Delta^{\prime} \in \mathcal{F}(H)}, \text { com } b_{\Delta^{\prime}}=f_{\Delta^{\prime}}\left(a_{\Delta}\right), \quad \Delta^{\prime} \in \mathcal{F}(H) \text { e } \Delta=f^{*}\left(\Delta^{\prime}\right),
$$

onde $f_{\Delta^{\prime}}: G / \Delta \longrightarrow H / \Delta^{\prime}$ é o morfismo derivado de $f(5.51,4.5)$.

Prova. Fixado um SG-morfismo $f: G \longrightarrow H$ de grupos especiais reduzidos, 5.51 mostra que

$$
\alpha_{f}: \mathcal{G} \longrightarrow \mathcal{H}
$$

é um morfismo de troca de base do sistema cofiltrante $\mathcal{G}$ sobre $\mathcal{F}(G)$ associado a $G$ no correspondente sistema $\mathcal{H}$ associado a $H$. Pela Proposição 5.34, existe um único SG-morfismo contínuo

$$
\mathcal{P}(f)={ }_{\text {def }} \quad f_{\alpha_{f}}: \mathcal{P}(G) \longrightarrow \mathcal{P}(H),
$$

tal que para $\Delta^{\prime} \in \mathcal{F}(H)$ e $\Delta=f^{*}\left(\Delta^{\prime}\right) \in \mathcal{F}(G)$, faz comutar o seguinte diagrama :

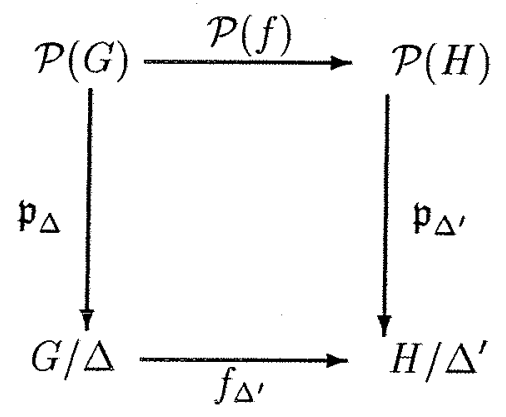


$O$ fato que $\mathcal{P}$ é um funtor segue imediatamente de 5.34.(b) e das relações (comp) e (ident) em 5.51 .

Se $a \in \mathcal{P}(G)$, a comutatividade do diagrama acima acarreta que para todo $\Delta^{\prime} \in \mathcal{F}(H)$, se $\Delta=$ $f^{*}\left(\triangle^{\prime}\right)$, então

$$
\mathfrak{p}_{\Delta^{\prime}}(\mathcal{P}(f)(a))=f_{\Delta^{\prime}}\left(\mathfrak{p}_{\Delta}(a)\right)=f_{\Delta^{\prime}}\left(a_{\Delta}\right)
$$

estabelecendo a fórmula desejada para $\mathcal{P}(f)(a)$; a definição de $f_{\Delta^{\prime}}: G / \Delta \longrightarrow H / \Delta^{\prime}(4.4)$ garante que para todo $u \in G$ temos

$$
f_{\Delta^{\prime}}(u / \Delta)=f(u) / \Delta^{\prime}
$$

Logo, para $u \in G$, a expressão para $\mathcal{P}(f)$ e a igualdade acima fornecem

$$
\mathcal{P}(f)\left(\eta_{G}(u)\right)=\left\langle f(u) / \Delta^{\prime}\right\rangle_{\Delta^{\prime} \in \mathcal{F}(H)}=\eta_{H}(f(u))
$$

completando a prova.

\section{Observação $\mathbf{5 . 5 3}$.}

a) O quadrado comutativo no enunciado do Teorema 5.52 mostra que $\eta$ é uma transformação natural do funtor identidade de RSG para o funtor $\mathcal{P}$.

b) A propriedade de unicidade em 5.43.(b) mostra que :

* O funtor $\mathcal{P}$ é adjunto à esquerda do funtor esquecimento de $\mathbf{R S G}_{p f}$ para $\mathbf{R S G}$;

* Para cada SG-morfismo de grupos reduzidos, $f: G \longrightarrow H$, existe em um único SG-morfismo contínuo - indicado por $\mathcal{P}(f)$-, que faz o quadrado no enunciado de 5.52 comutativo.

O restante desta seção será dedicado à prova de que o funtor $\mathcal{P}$ preserva quocientes por subgrupos saturados, ou seja do seguinte

Teorema 5.54. Seja $G$ um grupo especial reduzido e $\Sigma$ um subgrupo saturado de $G$. Seja

$$
\mathcal{M}=\left\{\sigma \in X_{G}: \Sigma \subseteq \operatorname{ker} \sigma\right\} .
$$

Com a notaçấo em 5.43.(b), seja $\bar{\Sigma}=\bigcap\{$ ker $\tilde{\sigma}: \sigma \in \mathcal{M}\}$. Então :

a) $\bar{\Sigma}$ é um subgrupo saturado e fechado em $\mathcal{P}(G)$, que é interseção de subgrupos saturados de índice 2, abertos e fechados em $\mathcal{P}(G)$. Além disso, com a topologia quociente, $\mathcal{P}(G) / \bar{\Sigma}$ é um grupo especial reduzido Booleano. ${ }^{38}$

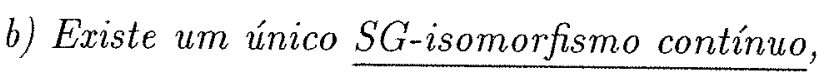

$$
g: \mathcal{P}(G / \Sigma) \longrightarrow \mathcal{P}(G) / \bar{\Sigma}
$$

que faz comutar o seguinte diagrama:

\footnotetext{
${ }^{38}$ Note que $\bar{\Sigma}$ tem a [pst], conforme 5.31.(ii).
} 


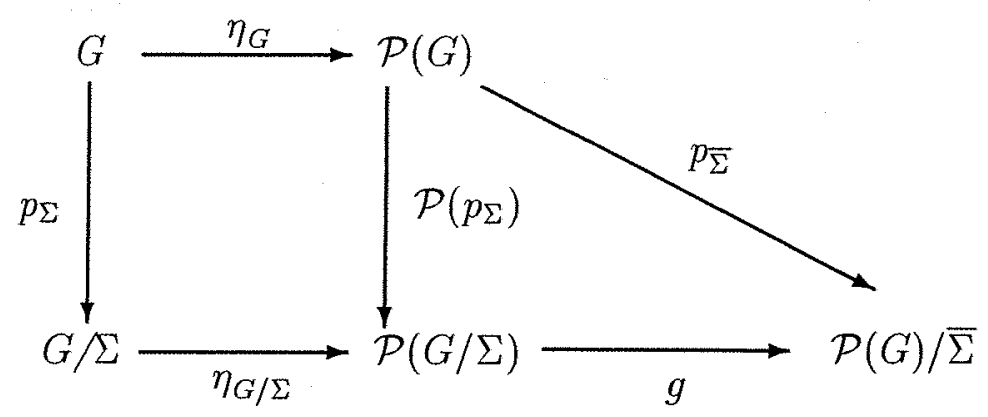

Prova. O item (a) é consequência imediata do Corolário 5.47 e do Teorema 5.28. O núcleo do resultado é o item (b) : a existência do $\mathrm{SG}$-isomorfismo $g$, com as propriedades desejadas. Iremos utilizar o critério em 5.43.(c). Consideremos a composição

$$
G \stackrel{\eta_{G}}{\longrightarrow} \mathcal{P}(G) \stackrel{p_{\bar{\Sigma}}}{\longrightarrow} \mathcal{P}(G) / \bar{\Sigma} .
$$

Então

$$
\operatorname{ker}\left(p_{\bar{\Sigma}} \circ \eta_{G}\right)=\Sigma \text {. }
$$

De fato, a afirmação acima é equivalente à $\eta_{G}^{-1}(\bar{\Sigma})=\Sigma$. Pela construção de $\bar{\Sigma}$ temos

$$
\bar{\Sigma}=\bigcap_{\sigma \in \mathcal{M}} \operatorname{ker} \tilde{\sigma} \text {. }
$$

Lembrando que para $\sigma \in \mathcal{M}$, temos $\sigma=\tilde{\sigma} \circ \eta_{G}$, concluímos que para todo $a \in G$ e $\sigma \in \mathcal{M}$,

$$
\sigma(a)=1 \quad \Leftrightarrow \quad \tilde{\sigma}\left(\eta_{G}(a)\right)=1 \Leftrightarrow \eta_{G}(a) \in \operatorname{ker} \widetilde{\sigma} .
$$

Assim $\eta_{G}^{-1}(\operatorname{ker}(\widetilde{\sigma}))=\operatorname{ker}(\sigma), \sigma \in \mathcal{M}$. Uma vez que, por 3.30.(c) temos $\Sigma=\bigcap \mathcal{M}$, a relação em (I) segue imediatamente.

Pelo Teorema 3.29.(c), existe um único SG-morfismo injetor, $h: G / \Sigma \longrightarrow \mathcal{P}(G) / \bar{\Sigma}$, tal que o seguinte diagrama é comutativo:

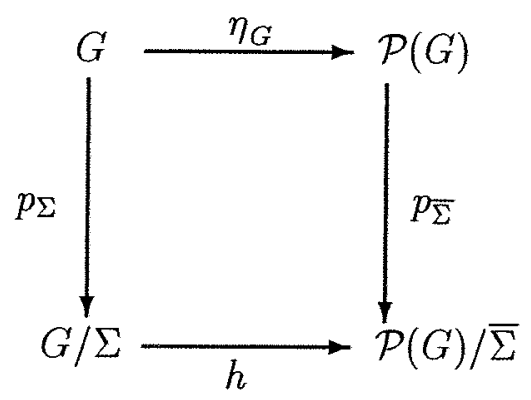

Mostremos que o morfismo $h$ tem as propriedades (1) e (2) em 5.43.(c).

(1) $\eta_{G / \Sigma}$ é contínua na topologia induzida por $\mathcal{P}(G) / \bar{\Sigma}$ em $G / \Sigma$ via $h$.

Pelo Fato 5.30.(b), o conjunto

$$
\mathcal{N}=\left\{\left(\operatorname{ker} \widetilde{\sigma}_{1} \cap \ldots \cap \operatorname{ker} \widetilde{\sigma}_{n}\right) / \bar{\Sigma}:\left\{\sigma_{1}, \ldots, \sigma_{n}\right\} \subseteq \mathcal{M}\right\}
$$

é um pf-sistema em $\mathcal{P}(G) / \bar{\Sigma}$ na topologia quociente.

Note que para $\left\{\sigma_{1}, \ldots, \sigma_{n}\right\} \subseteq \mathcal{M}$, temos 


$$
h^{-1}\left(\left(\operatorname{ker} \widetilde{\sigma}_{1} \cap \ldots \cap \operatorname{ker} \widetilde{\sigma}_{n}\right) / \bar{\Sigma}\right)=\left(\operatorname{ker} \sigma_{1} \cap \ldots \cap \operatorname{ker} \sigma_{n}\right) / \Sigma .
$$

De fato, sejam $\Delta=\bigcap_{i=1}^{n}$ ker $\sigma_{i}$ e $\tilde{\Delta}=\bigcap_{i=1}^{n}$ ker $\dot{\sigma}_{j}$. Como $p_{\bar{\Sigma}}$ é um morfismo de grupos e $\bar{\Sigma} \subseteq \widetilde{\Delta}$, temos $^{39}$

$$
\widetilde{\Delta}=p_{\bar{\Sigma}}^{-1}(\widetilde{\Delta} / \bar{\Sigma})
$$

Logo, segue de (II) e da comutatividade do diagrama em $(\bar{\Sigma})$ que

$$
\Delta=\eta_{G}^{-1}(\widetilde{\Delta})=\eta_{G}^{-1}\left(p_{\bar{\Sigma}}^{-1}(\widetilde{\Delta} / \bar{\Sigma})\right)=p_{\Sigma}^{-1}\left(h^{-1}(\widetilde{\Delta} / \bar{\Sigma})\right)
$$

Como $p_{\Sigma}$ é sobrejetora ${ }^{40}$, a igualdade acima acarreta (III), como desejado. Assim, na topologia induzida por $\mathcal{P}(G) / \bar{\Sigma}$ em $G / \Sigma$ via $h$, o conjunto

$$
\mathcal{V}=\left\{\left(\operatorname{ker} \sigma_{1} \cap \ldots \cap \operatorname{ker} \sigma_{n}\right) / \Sigma:\left\{\sigma_{1}, \ldots, \sigma_{n}\right\} \subseteq \mathcal{M}\right\}
$$

é um sistema fundamental de vizinhanças abertas e fechadas (e de índice finito) de $1 \mathrm{em} G / \Sigma$. 41 Pela condição $\left(1^{*}\right)$ no item (c) da Observação 5.46 , a continuidade de $\eta_{G / \Sigma}$ em relação à topologia induzida por $\mathcal{P}(G) / \bar{\Sigma}$ em $G / \Sigma$ via $h$ é equivalente a

(IV) Se $\Gamma$ é um subgrupo saturado de índice finito em $G / \Sigma$, existe $V \in \mathcal{V}$ tal que $V \subseteq \Gamma$.

Isto é claro, pois o morfismo quociente $p_{\Sigma}: G \longrightarrow G / \Sigma$ induz bijeções inversas $\mathcal{F}(G / \Sigma) \rightleftarrows \mathcal{F}_{\Sigma}(G)$ (Corolário 4.13.(b)) e porque $\mathcal{F}_{\Sigma}(G)$ é o fecho por interseções finitas de $X_{\Sigma}(G)$ em $\operatorname{Ssat}(G)$ (Proposição 4.15.(c)).

Apresentamos também uma verificação explícita de (IV) :

Seja $\Gamma$ um subgrupo saturado de índice finito de $G / \Sigma$; então $H=(G / \Sigma) / \Gamma$ é um grupo especial reduzido e finito. Consequentemente, $X_{H} \subseteq \mathbb{Z}_{2}{ }^{H}$ é finito, digamos

$$
X_{H}=\left\{\tau_{1}, \ldots, \tau_{m}\right\} \text {. }
$$

Pelo Teorema de Separação (3.30.(c)), temos

$$
\{1 / H\}=\bigcap_{j=1}^{m} \operatorname{ker} \tau_{j}
$$

Para cada $1 \leq j \leq m$, sejam

$$
\left\{\begin{array}{rlrl}
\sigma_{j} & =\tau_{j} \circ p_{\Gamma} \circ p_{\Sigma} ; & G \stackrel{p_{\Sigma}}{\longrightarrow} G / \Sigma \stackrel{p_{\Gamma}}{\longrightarrow} H \stackrel{\tau_{j}}{\longrightarrow} \mathbb{Z}_{2} ; \\
\mu_{j}=\tau_{j} \circ p_{\Gamma} ; & G / \Sigma \stackrel{p_{\Gamma}}{\longrightarrow} H \stackrel{\tau_{j}}{\longrightarrow} \mathbb{Z}_{2} .
\end{array}\right.
$$

Então, $\sigma_{j} \in X_{G}$ e $\mu_{j} \in X_{G / \Sigma}, \quad 1 \leq j \leq m$. Note que temos

$$
\text { (VI) } \Sigma \subseteq \bigcap_{j=1}^{m} \operatorname{ker} \sigma_{j} ; \quad \text { (VII) } \Gamma=\bigcap_{j=1}^{m} \operatorname{ker} \mu_{j} .
$$

A relação (VI) está clara, enquanto que a igualdade em (VII) segue imediatamente de (V). De (VI) obtemos $\left\{\sigma_{1}, \ldots, \sigma_{m}\right\} \subseteq \mathcal{M}$; por outro lado, se $\Delta=\bigcap_{j=1}^{m} \operatorname{ker} \sigma_{j}$, então $\Delta / \Sigma \in \mathcal{V}$; por fim, (VII) e o fato que $\sigma_{j}=\mu_{j} \circ p_{\Sigma}, 1 \leq j \leq m$, acarretam $\Delta / \Sigma=\Gamma$, estabelecendo (IV) e completando a prova da condição (1).

\footnotetext{
${ }^{39}$ Veja o Corolário 4.13 .

${ }^{40} \mathrm{E}$ portanto, $p_{\Sigma}\left(p_{\Sigma}^{-1}(C)\right)=C, \forall C \subseteq G / \Sigma$.

${ }^{41}$ É um pf-sistema neste grupo especial e topológico, conforme a Definição 5.15 .
} 
(2) $h^{4}\left(\equiv_{G / \Sigma}\right)$ é densa em $\equiv_{\mathcal{P}(G) / \bar{\Sigma}}$

Sejam $a_{1}, a_{2}, a_{3}, a_{4} \in \mathcal{P}(G)$ tais que

$$
\left\langle t_{1} / \bar{\Sigma}, t_{2} / \bar{\Sigma}\right\rangle \equiv_{\mathcal{P}(G) / \bar{\Sigma}}\left\langle t_{3} / \bar{\Sigma}, t_{4} / \bar{\Sigma}\right\rangle .
$$

Por 3.29.(a), existe $u_{i} \in \mathcal{P}(G), 1 \leq i \leq 4$, tais que

$$
\text { (VIII) }\left\langle u_{1}, u_{2}\right\rangle \equiv_{\mathcal{P}(G)}\left\langle u_{3}, u_{4}\right\rangle \quad \text { e } \quad(\mathrm{IX}) \quad p_{\bar{\Sigma}}\left(u_{i}\right)=a_{i} / \bar{\Sigma}, 1 \leq i \leq 4 .
$$

Seja $W$ uma vizinhança aberta de $1 \mathrm{em} \mathcal{P}(G) / \bar{\Sigma}$ na topologia quociente. Com a notação em 5.41 , como $p_{\bar{\Sigma}}$ é contínua $(5.28)$ e

$$
T=\left\{\operatorname{ker} \mathfrak{p}_{\Delta}: \Delta \in \mathcal{F}(G)\right\}
$$

é um pf-sistema em $\mathcal{P}(G)$, existe $\Delta \in \mathcal{F}(G)$ tal que

$$
\operatorname{ker}\left(\mathfrak{p}_{\Delta}\right) \subseteq p_{\bar{\Sigma}}^{-1}(W)
$$

Consideremos

$$
V=u_{1} k e r \mathfrak{p}_{\Delta} \times u_{2} k e r \mathfrak{p}_{\Delta} \times u_{3} k e r \mathfrak{p}_{\Delta} \times u_{4} k e r \mathfrak{p}_{\Delta}
$$

que é uma vizinhaça aberta e fechada de $\left\langle u_{1}, u_{2}, u_{3}, u_{4}\right\rangle$ em $\mathcal{P}(G)^{4}$.

Note que (IX) e (X) implicam

$$
\left(p_{\bar{\Sigma}}\right)^{4}(V) \subseteq t_{1} W \times t_{2} W \times t_{3} W \times t_{4} W .
$$

Uma vez que a imagem da isometria em $G$ é densa na isometria de $\mathcal{P}(G)(5.45)$, existem $a_{i} \in G$, $1 \leq i \leq 4$, tais que

$$
\text { (XII) }\left\langle a_{1}, a_{2}\right\rangle \equiv_{G}\left\langle a_{3}, a_{4}\right\rangle \quad \text { e } \quad(\mathrm{XIII})\left\langle\eta_{G}\left(a_{1}\right), \eta_{G}\left(a_{2}\right), \eta_{G}\left(a_{3}\right), \eta_{G}\left(a_{4}\right)\right\rangle \in V .
$$

Então temos :

* De (XII) segue que $\left\langle a_{1} / \Sigma, a_{2} / \Sigma\right\rangle \equiv_{G / \Sigma}\left\langle a_{3} / \Sigma, a_{4} / \Sigma\right\rangle$

* Da comutatividade do diagrama $(\bar{\Sigma})$, de $(\mathrm{XI})$ e de (XIII) obtemos

$$
\left\langle h\left(a_{1} / \Sigma\right), h\left(a_{2} / \Sigma\right), h\left(a_{3} / \Sigma\right), h\left(a_{4} / \Sigma\right)\right\rangle \in t_{1} W \times t_{2} W \times t_{3} W \times t_{4} W
$$

completando a prova da condição (2).

Pelo item (c) do Teorema 5.43,

$$
g=_{\text {def }} \widetilde{h}: \mathcal{P}(G / \Sigma) \longrightarrow \mathcal{P}(G) / \bar{\Sigma}
$$

é o único SG-isomorfismo contínuo, que faz comutar o primeiro diagrama abaixo :
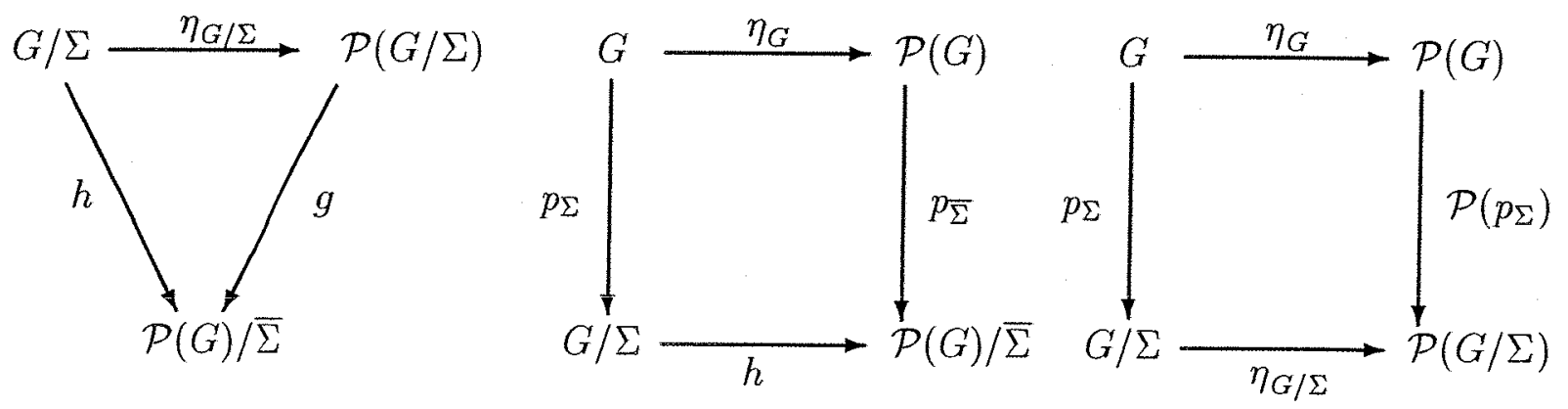
O diagrama do meio é o mesmo de $(\bar{\Sigma})$ acima, enquanto que o derradeiro vem do fato que a associação $H \mapsto \eta_{H}$ define uma transformação natural do funtor identidade de RSG para o funtor $\mathcal{P}(5.53)$. Esta repetição facilitará a compreensão da prova de que o diagrama do enunciado é comutativo:

Como o quadrado que aparece no enunciado é comutativo, é suficiente verificar que

$$
p_{\bar{\Sigma}}=g \circ \mathcal{P}\left(p_{\Sigma}\right) \text {. }
$$

Utilizando a comutatividade dos diagramas acima, obtemos

$$
p_{\bar{\Sigma}} \circ \eta_{G}=h \circ p_{\Sigma}=g \circ \eta_{G / \Sigma} \circ p_{\Sigma}=g \circ \mathcal{P}\left(p_{\Sigma}\right) \circ \eta_{G} .
$$

Uma vez que $p_{\bar{\Sigma}}$ e $g \circ \mathcal{P}\left(p_{\Sigma}\right)$ são contínuos, com valores em um espaço Hausdorff, e que $\eta_{G}$ tem imagem densa em $\mathcal{P}(G)$ (5.43.(a)), a igualdade acima implica que $p_{\bar{\Sigma}}=g \circ \mathcal{P}\left(p_{\Sigma}\right)$, completando a prova.

A partir do Teorema 5.54, passaremos a identificar $\mathcal{P}(G / \Sigma)$ com $\mathcal{P}(G) / \bar{\Sigma}$, escrever os diagramas associados neste caso da seguinte forma :
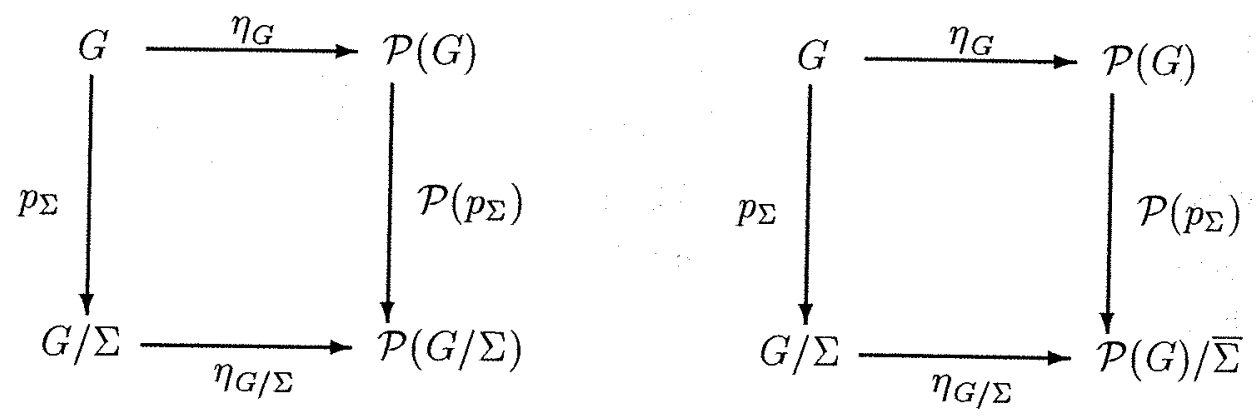

\subsection{Extensão ao caso Hausdorff}

Para finalizar este Capítulo, descrevemos como muitas das construções e resultados referentes as envoltórias profinitas de grupos especiais reduzidos (discretos) podem ser estendidos para o caso dos grupos especiais reduzidos e Hausdorffs.

Recordemos:

\subsection{Complementos de topologia :}

(i) Seja $X, Y$ espaços topológicos e $f: X \rightarrow Y$ uma função contínua e sobrejetora. $Y$ possui a topologia quociente determinada por $f$ sse

Para cada $V \subseteq Y, V$ é aberto (respec. fechado) em $Y$ sse $f^{-1}[V]$ é aberto (respec. fechado) em $X$.

(ii) Se $f: X \rightarrow Y$ é uma função contínua e sobrejetora que é também aberta (respec. fechada) então $Y$ detém a topologia quociente. 
(iii) Se $X$ é um espaço compacto e $Y$ um espaço Hausdorff então toda função contínua $f: X \rightarrow Y$ é uma função fechada.

(iv) Toda função fechada com imagem densa é obviamente sobrejetora.

\subsubsection{Sobre os Grupos Especiais Topológicos}

5.56. Categorias de grupos especiais topológicos : Iniciamos recordando a Definição 5.20

(i) $\mathbf{R S G}^{\text {top }}$ é a categoria cujos objetos são os grupos topológicos que são especiais e reduzidos e com morfismos os $R S G$-morfismos contínuos.

(ii) $\mathbf{R S G}{ }^{\text {Haus }}$ é a subcategoria plena de $\mathbf{R} \mathbf{S G}^{\text {top }}$ constituída dos grupos especiais reduzidos e que são grupos topológicos Hausdorffs.

(iii) $\mathbf{R S G}^{\text {disc }}$ é a subcategoria plena de $\mathbf{R S G}^{t o p}$ constituída dos grupos especiais reduzidos e que são grupos topológicos discretos.

(iv) $\mathbf{R S G}_{f i n}$ é a subcategoria plena de $\mathbf{R S G}^{\text {top }}$ constituída dos grupos especiais reduzidos finitos e que são grupos topológicos discretos.

(v) $\mathbf{R S G}_{p f}$ é a subcategoria plena de $\mathbf{R S G}^{\text {top }}$ constituída dos grupos especiais reduzidos profinitos e que são grupos topológicos quando munidos da topologia booleana herdada.

Conforme observamos (5.21) podemos identificar as categorias RSG e $\mathbf{R S G}^{\text {disc }}$ e considerar $\mathbf{R S G}_{f i n}$ como subcategoria plena tanto de $\mathbf{R S G}$ como de $\mathbf{R S G}_{p f}$.

\subsection{Morfismos Derivados são contínuos : ${ }^{42}$}

Seja $(G \stackrel{f}{\rightarrow} H) \in \mathbf{R S G}^{\text {top }}$. Para cada $\Sigma \in S$ sat $(G)$ e $\Delta^{\prime} \in S \operatorname{sat}(H)$ tais que $\Sigma \subseteq f^{*}\left(\Delta^{\prime}\right)$ vemos, por caça ao diagrama, que o morfismo derivado $f_{\Sigma, \Delta^{\prime}}: G / \Sigma \rightarrow H / \Delta^{\prime}$, o único $R S G$-morfismo tal que

$$
\left(G \stackrel{f}{\rightarrow} H \stackrel{p_{\Delta^{\prime}}}{\rightarrow} H / \Delta^{\prime}\right)=\left(G \stackrel{p_{\Sigma}}{\rightarrow} G / \Sigma \stackrel{f_{\Sigma, \Delta^{\prime}}}{\rightarrow} H / \Delta^{\prime}\right)
$$

é sempre contínuo quando tomamos os grupos quocientes munidos da topologia quociente.

\footnotetext{
${ }^{42}$ Veja 4.4 e 4.5
} 


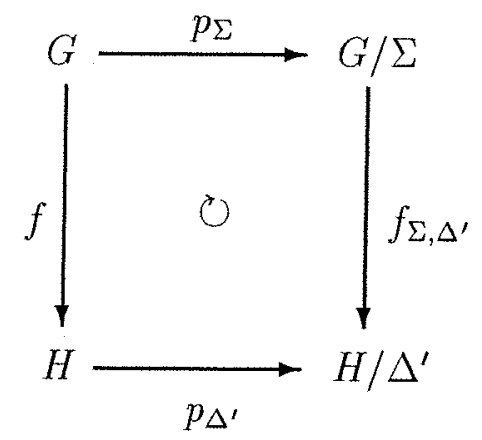

Onde $\left(\Sigma \subseteq f^{*}\left(\Delta^{\prime}\right)\right) \in S$ sat $(G)$

5.58. Sobre quocientes em RSG ${ }^{t o p}$ : Segue das bijeções em 4.13 e da definição de topologia quociente que:

(i) Para cada $G \in \mathbf{R S G}^{\text {top }}$ e cada $\Delta \in S$ sat $(G)$ temos bijeções:

* $\operatorname{Ssat}(G / \Delta) \cap T(G / \Delta) \rightleftarrows \operatorname{Ssat}_{\Delta}(G) \cap T(G)$

* $\operatorname{Ssat}^{\star}(G / \Delta) \cap T(G / \Delta) \rightleftarrows S s a t_{\Delta}^{\star}(G) \cap T(G)$

* $\mathcal{F}(G / \Delta) \cap T(G / \Delta) \rightleftarrows \mathcal{F}_{\Delta}(G) \cap T(G)$

* $X(G / \Delta) \cap T(G / \Delta) \rightleftarrows X_{\Delta}(G) \cap T(G)$

Onde, para cada espaço $Y$ tomamos $T(Y) \in\{O$ pen $(Y), \operatorname{Closed}(Y), \operatorname{Clopen}(Y)\}$.

(ii) Duplo quociente é quociente : Para cada $(\Delta \subseteq \Sigma) \in S$ sat $(G) \cap T(G)$ o RSG ${ }^{\text {top }}$-morfismo obtido de $p_{\Delta, \Sigma}: G / \Delta \rightarrow G / \Sigma$ por passagem ao quociente $\overline{p_{\Delta, \Sigma}}:(G / \Delta) /(\Sigma / \Delta) \longrightarrow G / \Sigma$ é um $\mathbf{R S G}^{\text {top }}$-isomorfismo.

\subsection{Considerando $\mathcal{V}$ :}

* Seja $G$ um grupo especial reduzido e topológico e consideremos

$$
\mathcal{V}(G)=\{\Delta \in \mathcal{F}(G): \Delta \text { é aberto em } G\} .
$$

Então $\mathcal{V}(G)=\mathcal{F}(G) \cap C l o p e n(G)$, munido da inclusão, é um conjunto ordenado dirigido para baixo.

Além disso, se $G$ admite algum pf-sistema (Definição 5.15) então $\mathcal{V}(G)$ é o maior deles (Observação 5.16.(i)) .

* Sejam $G, H \in O b j\left(\mathbf{R S G}{ }^{\text {top }}\right)$ e $f: G \longrightarrow H$ um $\mathbf{R S G}^{\text {top }}$-morfismo (i.e. um $R S G$-morfismo contínuo) temos função bem definida e crescente $\alpha_{f}: \mathcal{V}(H) \longrightarrow \mathcal{V}(G)$ dada por $\Delta^{\prime} \mapsto f^{*}\left(\Delta^{\prime}\right)=$ $f^{-1}\left[\Delta^{\prime}\right]$.

* Assim obtemos um funtor contravariante :

$$
\mathcal{V}: \mathbf{R S G}^{\text {top }} \longrightarrow \text { POD }
$$

onde POD designa a categoria dos conjuntos ordenados dirigidos para baixo e funçôes crescentes. 


\subsubsection{Sobre RSG $^{\text {Haus }}$}

5.60. Sobre quocientes em $\mathrm{Gr}^{\text {Haus }}$ : Recordamos o item (1) do Lema 5.3.(b) :

Para cada grupo topológico Hausdorff $G$ temos que:

* Se $N$ é um subgrupo normal fechado de $G$, o grupo quociente $G / N$, munido da topologia quociente é um grupo topológico Hausdorff.

* Em particular, se $N$ é um subgrupo normal fechado (e/ou aberto) com índice finito em $G$ então $G / N$ é um grupo topológico finito e discreto.

As colocações acima nos dizem, em particular, que:

$\left(^{*}\right)$ Se $G, H \in \mathbf{R S G}^{\text {Haus }}$ e $f: G \longrightarrow H$ é um $\mathbf{R S G}^{\text {Haus }}$-morfismo então, para cada $\Delta^{\prime} \in \mathcal{V}(H)$, temos $f^{*}\left(\Delta^{\prime}\right) \in \mathcal{V}(G)$ e o morfismo derivado $f_{\Delta^{\prime}}: G / f^{*}\left(\Delta^{\prime}\right) \longmapsto H / \Delta^{\prime}$ é um RSG $^{\text {top }}{ }_{\text {-morfismo }}$ de grupos finitos e discretos $\left(\mathbf{R S G}_{\text {fin }}\right.$-morfismo) .

E assim, como em 5.51 temos um funtor, da categoria dos grupos topológicos Hausdorffs que são especiais e reduzidos e SG-morfismos contínuos, para a categoria dos diagramas cofiltrantes de grupos especiais reduzidos finitos e discretos e morfismos de troca de base (Definição 5.33).

Explicitamente: ${ }^{43}$

5.61. O funtor "pro-diagrama associado" : $\Upsilon: \mathbf{R S G}$ Haus $\longrightarrow \operatorname{Diag}_{\Omega}\left(\mathbf{R S G}_{p f}\right)$, onde $\Omega=$ POD, a categoria dos conjuntos ordenados dirigidos para baixo e funções crescentes.

Objetos: Para cada objeto $G \in \mathbf{R S G}^{\text {Haus }}$ tomamos: $\Upsilon(G) \doteq(\mathcal{V}(G), D(G))$.

Onde: $\quad D(G): \mathcal{V}(G) \longrightarrow \mathbf{R S G}_{p f}:(\Delta \subseteq \Sigma) \mapsto\left(G / \Delta \stackrel{p_{\Delta, \Sigma}}{\rightarrow} G / \Sigma\right)$.

(Notemos que: $D(G): \mathcal{V}(G) \longrightarrow \mathbf{R S G}_{f i n} \subseteq \mathbf{R S G}_{p f} \cdot$ )

Morfismos : Para cada flecha $\left(G \stackrel{f}{\rightarrow} G^{\prime}\right) \in \mathbf{R S G}^{\text {Haus }}$ tomamos: $\Upsilon(f) \doteq(\mathcal{V}(f), \Phi(f))$.

Onde: $\quad \Phi(f): D(G) \circ \mathcal{V}(f) \longrightarrow D\left(G^{\prime}\right): \Delta^{\prime} \mapsto \Phi(f)_{\Delta^{\prime}}=\left(G / f^{*}\left(\Delta^{\prime}\right) \stackrel{f_{\Delta^{\prime}}}{\longrightarrow} G^{\prime} / \Delta^{\prime}\right)$ é a transformação natural "derivada" entre diagramas indexados por $\mathcal{V}\left(G^{\prime}\right)$.

\footnotetext{
${ }^{43} \mathrm{Na}$ notação da subseção 1.2 .5 .
} 


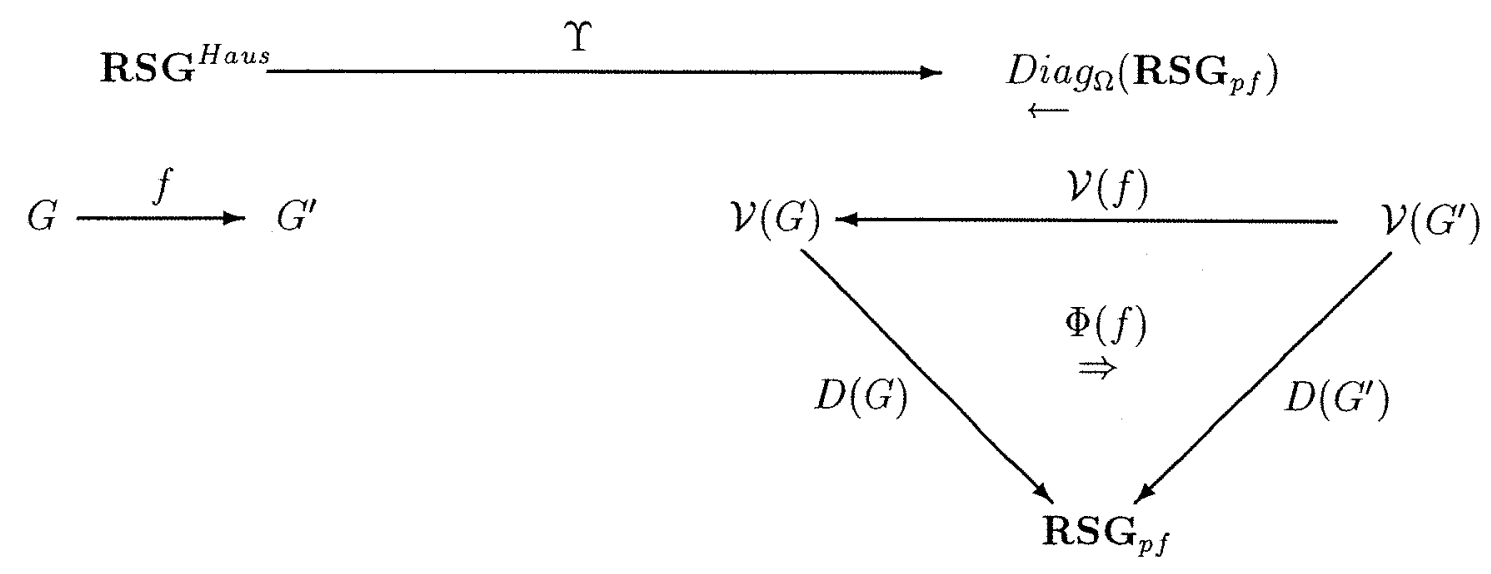

Vimos, no decorrer do Capítulo, que $\mathbf{R S G}_{p f}$ é fechada por limites projetivos cofiltrantes em RSG ${ }^{\text {top }}$ por isso pudemos definir o "funtor limite" (Proposição 5.34)

$$
L: \operatorname{Diag}_{\Omega}\left(\mathbf{R S G}_{p f}\right) \longrightarrow \mathbf{R S G}_{p f} \cdot{ }^{44}
$$

Seja $\mathcal{P}: \mathbf{R S G}^{\text {Haus }} \longrightarrow \mathbf{R S G}_{p f}$ a composição

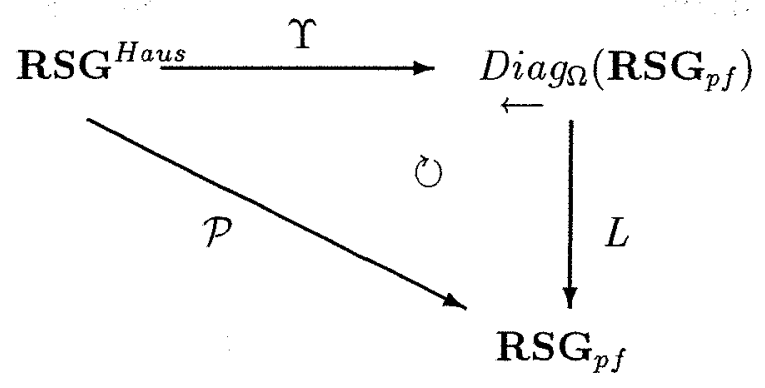

Isto é, $\mathcal{P}: \mathbf{R S G}^{\text {Haus }} \longrightarrow \mathbf{R S G}_{p f}$ é o

\subsubsection{O funtor "Envoltória Especial Profinita"}

5.62. Escrevendo $\mathcal{P}: \mathbf{R S G}^{\text {Haus }} \longrightarrow \mathbf{R S G}_{p f}:{ }^{45}$

Objetos : $\quad G \in \mathbf{R S G}^{\text {Haus }} \mapsto \mathcal{P}(G) \doteq \underset{\Delta \in \overleftarrow{\mathcal{V}}_{(G)}}{\lim } G / \Delta \in \mathbf{R S G}_{p f}$

\footnotetext{
${ }^{44} \mathrm{Na}$ notação da Proposição 1.55 .
}

${ }^{45}$ Veja o Teorema 5.52 . 
E escrevemos $\left(\mathcal{P}(G) \stackrel{\mathfrak{g}_{\Delta}}{\longrightarrow} G / \Delta: \Delta \in \mathcal{V}(G)\right)$, o cone limite.

Morfismos : $\quad\left(G \stackrel{f}{\longrightarrow} G^{\prime}\right) \in \mathbf{R S G}^{\text {Haus }} \mapsto\left(\mathcal{P}(G) \stackrel{\mathcal{P}(f)}{\longrightarrow} \mathcal{P}\left(G^{\prime}\right)\right) \in \mathbf{R S G}_{p f}$,

$$
\left(g_{\Delta} / \Delta\right)_{\Delta \in \mathcal{V}(G)} \in \mathcal{P}(G) \quad \mapsto \quad\left(f\left(g_{f^{*}\left(\Delta^{\prime}\right)}\right) / \Delta^{\prime}\right)_{\Delta^{\prime} \in \mathcal{V}\left(G^{\prime}\right)} \in \mathcal{P}\left(G^{\prime}\right)
$$

\subsection{A transformação natural diagonal :}

* Para cada $G \in \mathbf{R S G}^{\text {Haus }}$, o cone projetivo $\left(G \stackrel{p_{\Delta}}{\longrightarrow} G / \Delta\right)_{\Delta \in \mathcal{V}(G)}$ origina um único $\mathbf{R S G}^{\text {Haus }}$ morfismo ("diagonal") :

$$
g \in G \quad \mapsto \quad \eta_{G}(g)=(g / \Delta)_{\Delta \in \mathcal{V}_{(G)}} \in \mathcal{P}(G)
$$

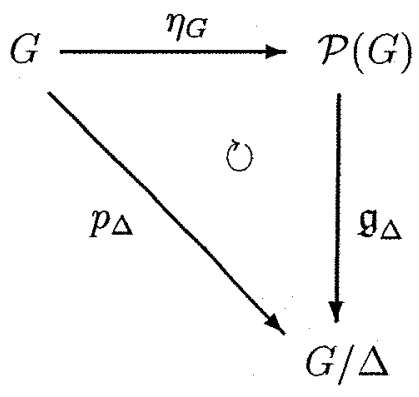

* Mantendo a convenção de, por simplicidade, não escrever o funtor inclusão $\iota: \mathbf{R S G}_{p f} \hookrightarrow$ $\mathbf{R S G}^{\text {Haus }}$, e então considerar $\mathcal{P}$ também como um funtor $\mathbf{R S G}^{\text {Haus }} \longrightarrow \mathbf{R S G}^{\text {Haus }}$, temos que a associação

$$
G \mapsto\left(\eta_{G}: G \longrightarrow \mathcal{P}(G)\right)
$$

constitui uma transformação natural do funtor identidade de $\mathbf{R S G}^{\text {Haus }}$ para o funtor $\mathcal{P}$.

* Do mesmo modo que em 5.43.(a) verifica-se que, para cada $G \in \mathbf{R S G}^{\text {Haus }}, \eta_{G}: G \longrightarrow \mathcal{P}(G)$ tem imagem densa. ${ }^{46}$

* Se $G \in \mathbf{R S G}^{\text {Haus }}$ admitir algum pf-sistema então o $S G$-morfismo contínuo $\eta_{G}$ é injetor ${ }^{47} \mathrm{e}$ a topologia original de $G$ coincide com a topologia de subespaço induzida por $\eta_{G}{ }^{48}$.

* Se $H \in \mathbf{R S G}_{p f}$ então segue da Observação 5.18 que $\eta_{H}: H \longrightarrow \mathcal{P}(H)$ é um $\mathbf{R S G}_{p f^{-}}$ isomorfismo.

\footnotetext{
${ }^{46}$ Veja também o Lema 5.6 .

${ }^{47} \mathrm{Se} G \in \mathbf{R S G}^{\text {Haus }}$ admite pf-sistema (respec. pf-sistema que "decide uma fórmula p.p." $\phi$ (principio localglobal)) então $\eta_{G}$ é injetor (respec. é injetor e reflete a validade da fórmula p.p. $\phi$ ).

${ }^{48}$ Veja o Teorema 5.43.(a) e a Observação 5.46.(b) . Veja também a Observação 5.16.(iii).
} 


\subsubsection{Sobre a adjunção $\mathbf{R S G}^{\text {Haus }} \rightleftarrows \mathbf{R S G}_{p f}$}

Teorema 5.64. $\mathcal{P}: \mathbf{R S G}^{\text {Haus }} \longrightarrow \mathbf{R S G}_{p f}$ é um funtor "idempotente" e que é adjunto à esquerda do funtor inclusão $\iota: \mathbf{R S G}_{p f} \longrightarrow \mathbf{R S G}^{\text {Haus }}$.

Prova. Devemos verificar que, para cada $G \in \mathbf{R S G}^{\text {Haus }}$, cada $H \in \mathbf{R S G}_{p f}$ temos, que para cada $\mathbf{R S G}{ }^{\text {Haus }}$-morfismo $f: G \longrightarrow \iota(H)$ existe um único $\mathbf{R S G}_{p f}$-morfismo $\widetilde{f}: \mathcal{P}(G) \longrightarrow H$ tal que o diagrama abaixo comuta:

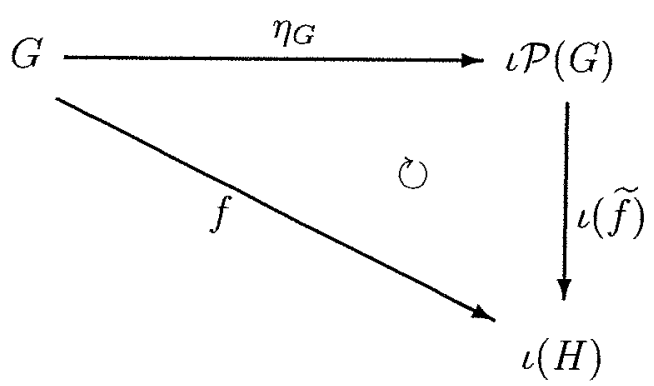

Existência : Porque o quadrado abaixo é comutativo e sua base é um isomorfismo.
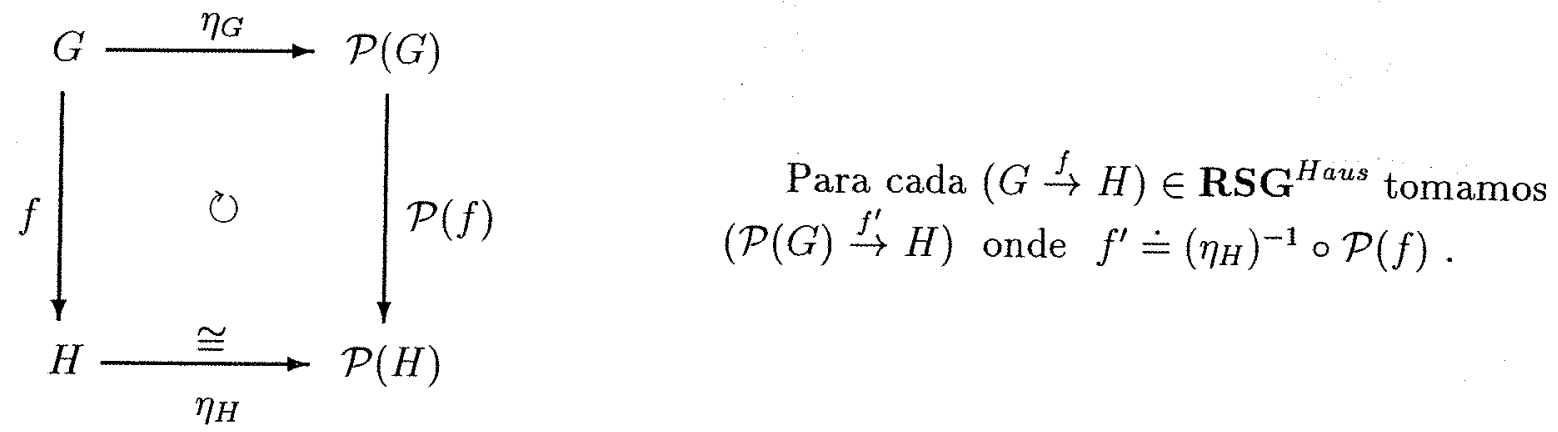
$\left(\mathcal{P}(G) \stackrel{f^{\prime}}{\rightarrow} H\right)$ onde $f^{\prime} \doteq\left(\eta_{H}\right)^{-1} \circ \mathcal{P}(f)$

\section{Unicidade :}

Para cada $f_{0}^{\prime}, f_{1}^{\prime} \in \mathbf{R S G}_{p f}(\mathcal{P}(G), H)$ consideremos:

$$
\left[f_{0}^{\prime}=f_{1}^{\prime}\right] \doteq\left\{x \in \mathcal{P}(G): f_{0}^{\prime}(x)=f_{1}^{\prime}(x)\right\} .
$$

Suponhamos que $f_{0}^{\prime}$ e $f_{1}^{\prime}$ sejam "extensões" de $f$, i.e., $f_{0}^{\prime} \circ \eta_{G}=f=f_{1}^{\prime} \circ \eta_{G}$.

Evidentemente temos: $\eta_{G}[G] \subseteq\left[f_{0}^{\prime}=f_{1}^{\prime}\right]$. Mas também fecho( $\left.\eta_{G}[G]\right) \subseteq\left[f_{0}^{\prime}=f_{1}^{\prime}\right]$ :

Porque $\mathcal{P}(G)$ é espaço Hausdorff e porque, por hipótese $f_{0}^{\prime}, f_{1}^{\prime}$ são funções contínuas, temos que $\left[f_{0}^{\prime}=f_{1}^{\prime}\right] \subseteq \mathcal{P}(G)$ é um fechado (e que contém a imagem de $\eta_{G}$ ).

Agora, como a imagem de $\eta_{G}$ é densa temos fecho $\left(\eta_{G}[G]\right)=\mathcal{P}(G), \operatorname{logo} \mathcal{P}(G) \subseteq\left[f_{0}^{\prime}=f_{1}^{\prime}\right] \subseteq \mathcal{P}(G)$ ou seja $f_{0}^{\prime}=f_{1}^{\prime}$.

Idempotência :

Como $\eta: I d_{\mathbf{R S G}}{ }^{\text {Haus }} \rightarrow \mathcal{P}$ é transformação natural entre endofuntores de $\mathbf{R S G}^{\text {Haus }}$ temos que o diagrama abaixo é comutativo: 


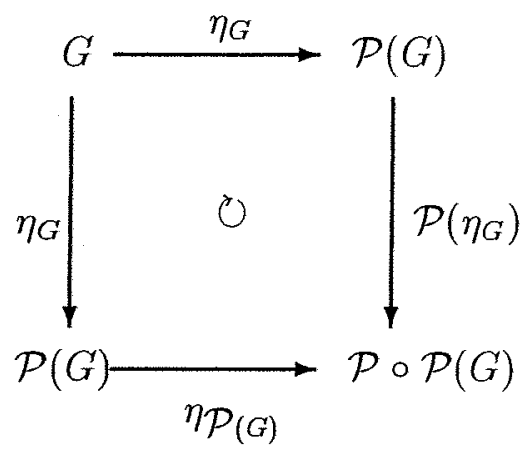

Como $\mathcal{P}(G) \in \mathbf{R S G}_{p f}$ temos que $\eta_{\mathcal{P}(G)}: \mathcal{P}(G) \stackrel{\cong}{\longrightarrow}(\mathcal{P} \circ \mathcal{P})(G)$ é $\mathbf{R S G}^{\text {Haus }}$-isomorfismo. Vimos acima que $\eta_{G}: G \longrightarrow \mathcal{P}(G)$ é universal, portanto $\mathcal{P}\left(\eta_{G}\right)=\eta_{\mathcal{P}(G)}$.

Assim ambas as transformações naturais de comparação $\eta \mathcal{P}, \mathcal{P} \eta: \mathcal{P} \longrightarrow \mathcal{P} \circ \mathcal{P}$ coincidem e são isomorfismos naturais de funtores.

\subsection{5 $\mathcal{P}$ e epimorfismos}

Nesta subseção a interação entre a topologia e a linguagem é determinante. O resultado central a ser apresentado nesta subseção é que o funtor $\mathcal{P}: \mathbf{R S G}^{\text {Haus }} \rightarrow \mathbf{R S G}_{p f}$ preserva quocientes.

Proposição 5.65. Para cada $(G \stackrel{f}{\rightarrow} H) \in \mathbf{R S G}^{\text {Haus }}$ temos:

(i) Se fé RSG-morfismo contínuo e denso então

* $\mathcal{P}(f)$ é RSG-morfismo contínuo, fechado, sobrejetor, e tal que a topologia em $\mathcal{P}(H)$ coincide com a topologia quociente obtida por $\mathcal{P}(f): \mathcal{P}(G) \rightarrow \mathcal{P}(H)$.

* $\operatorname{ker}(\mathcal{P}(f)) \subseteq \mathcal{P}(G)$ é um subgrupo saturado e fechado e $\mathcal{P}(G) /$ ker $(\mathcal{P}(f))$ é um grupo especial reduzido que é um grupo topológico booleano.

* A flecha obtida de $\mathcal{P}(f)$ por passagem ao quociente:

$$
\overline{\mathcal{P}(f)}: \mathcal{P}(G) / \operatorname{ker}(\mathcal{P}(f)) \mapsto \mathcal{P}(H)
$$

é um RSG-morfismo bijetor que é um isomorfismo de grupos topológicos booleanos.

(ii) $\mathcal{P}(f)$ é $\mathbf{R S G}_{p f}$-morfismo sobrejetor sse

Para cada $\left(h_{\Delta^{\prime}} / \Delta^{\prime}\right)_{\Delta^{\prime} \in \mathcal{V}(H)} \in \mathcal{P}(H)$ existe $\left(g_{\Delta} / \Delta\right)_{\Delta \in \mathcal{V}(G)} \in \mathcal{V}(G)$ tal que para cada $\Delta^{\prime} \in \mathcal{V}(H)$ temos $f\left(g_{f^{*}\left(\Delta^{\prime}\right)}\right) . h_{\Delta^{\prime}} \in \Delta^{\prime}$.

(iii) Se $\mathcal{P}(f)$ é $\mathbf{R S G}_{p f}$-morfismo sobrejetor então

Para cada $\Delta^{\prime} \in \mathcal{V}(H)$ temos que $f_{\Delta^{\prime}}: G / f^{*}\left(\Delta^{\prime}\right) \nrightarrow H / \Delta^{\prime}$ é $\mathbf{R S G}_{\text {fin }}$-morfismo bijetor.

\section{Prova.}

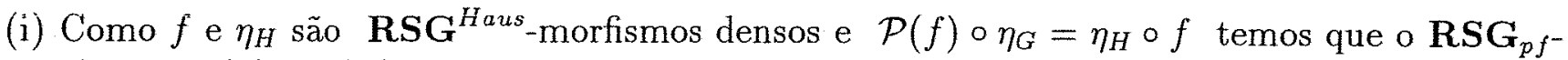
morfismo $\mathcal{P}(f): \mathcal{P}(G) \rightarrow \mathcal{P}(H)$ é denso, e sendo contínuo e fechado é portanto sobrejetor. 
Todas as outras observações seguem imediatamente do manejo das topologias envolvidas (ver observação sobre complementos de topologia 5.55)

(ii) É a decodificação da frase: "o $R S G$-morfismo $\mathcal{P}(f)$ é sobrejetor" !

(iii)

* Como o diagrama abaixo comuta e $p_{\Delta}: G \rightarrow G / \Delta$ é $\mathbf{R S G}_{p f}$-morfismo sobrejetor temos que $\mathfrak{g}_{\Delta}: \mathcal{P}(G) \rightarrow G / \Delta$ é $\mathbf{R S G}_{p f}$-morfismo sobrejetor.

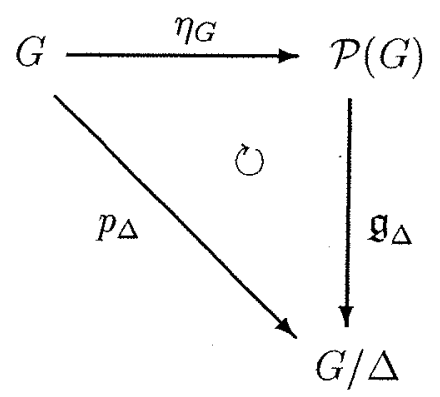

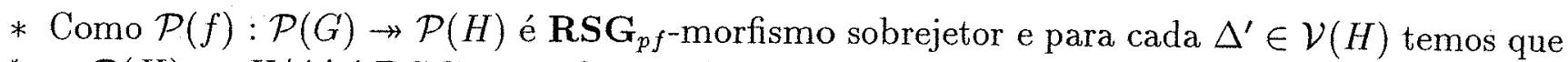

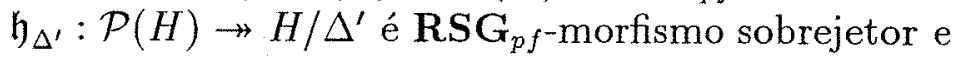

$$
f_{\Delta^{\prime}} \circ \mathfrak{g}_{f^{*}\left(\Delta^{\prime}\right)}=\mathfrak{h}_{\Delta^{\prime}} \circ \mathcal{P}(f)
$$

temos que o $\mathbf{R S G}_{f i n}$-morfismo injetor $f_{\Delta^{\prime}}: G / f^{*}(\Delta) \mapsto H / \Delta^{\prime}$ é também sobrejetor.

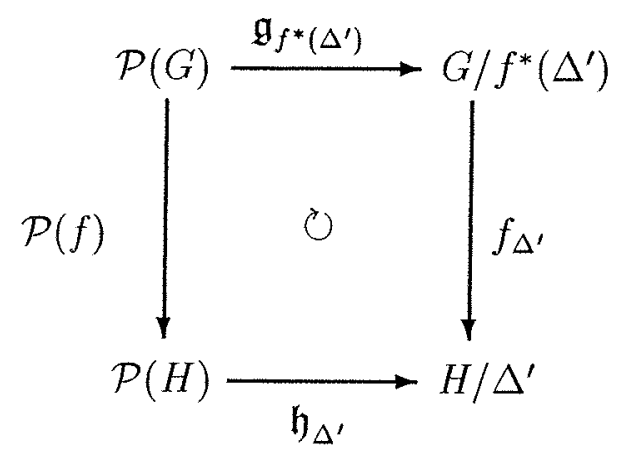

Para cada $\Delta^{\prime} \in \mathcal{V}(H)$

Verificaremos abaixo que o funtor $\mathcal{P}: \mathbf{R S G}^{\text {Haus }} \longrightarrow \mathbf{R S G}_{p f}$ preserva quocientes por subgrupos saturados e fechados. A verificação é extensa mas decidimos apresentá-la com um certo detalhe porque o método da prova correspondente no caso dos grupos reduzidos discretos (Teorema 5.54) não é, aparentemente, transponível para todos os quocientes por subgrupos saturados e fechados do grupo Hausdorff de partida. ${ }^{49}$ De fato a flecha natural de comparação é produzida

${ }^{49}$ Veja a Observação 5.31 e a 5.69 , no final desta seção. 
no sentido oposto ao de 5.54. ${ }^{50}$

Necessitaremos dos seguintes lemas:

Lema 5.66. Para $G \in \mathbf{R S G}^{\text {Haus }}$ temos:

(i) Para cada $\Delta \in \mathcal{V}(G)$ temos que a flecha obtida de

$$
\mathfrak{g}_{\Delta}: \mathcal{P}(G) \rightarrow G / \Delta:\left(g_{\Delta} / \Delta\right)_{\Delta \in \mathcal{V}(G)} \mapsto g_{\Delta} / \Delta
$$

por passagem ao quociente:

$$
\widehat{\mathfrak{g}_{\Delta}}:(\mathcal{P}(G)) / \operatorname{ker}\left(\mathfrak{g}_{\Delta}\right) \rightarrow G / \Delta
$$

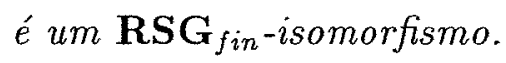

(ii) Para cada $(\Delta \subseteq \Sigma) \in \mathcal{V}(G)$ temos que $\left(\operatorname{ker}\left(\mathfrak{g}_{\Delta}\right) \subseteq \operatorname{ker}\left(\mathfrak{g}_{\Sigma}\right)\right) \in \mathcal{V}(\mathcal{P}(G))$ e que diagrama abaixo é comutativo:

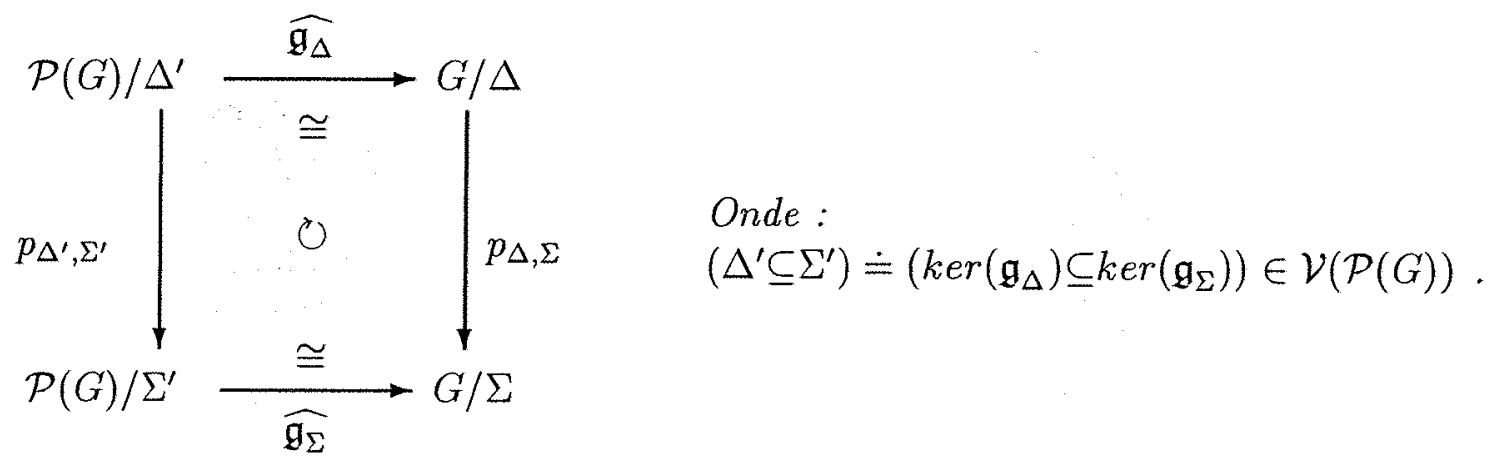

(iii) Para cada $\Theta \in S$ sat $(G)$ temos o $\mathbf{R S G}_{p f}$-isomorfismo:

$$
\underset{\Delta \in \operatorname{Vim}_{\Theta}(G)}{\lim } \widehat{\mathfrak{g}_{\Delta}}: \underset{\Delta \in \operatorname{V}_{\Theta}(G)}{\lim }(\mathcal{P}(G)) / \operatorname{ker}\left(\mathfrak{g}_{\Delta}\right) \stackrel{\cong}{\rightrightarrows} \lim _{\Delta \in \mathcal{V}_{\Theta}(G)}^{\leftarrow} G / \Delta .
$$

(iv) $X_{\mathcal{P}_{(G)}}=$ fecho( $\left.\bigcup_{\Delta \in \mathcal{V}_{(G)}} X_{\mathfrak{g}_{\Delta}}\left[X_{G / \Delta}\right]\right) \subseteq\left(\mathbb{Z}_{2}\right)^{\mathcal{P}(G)}$

\section{Prova.}

(i)

* $\widehat{\mathfrak{g}_{\Delta}}: \mathcal{P}(G) / \operatorname{ker}\left(\mathfrak{g}_{\Delta}\right) \nrightarrow G / \Delta$ é $\mathbf{R S G}_{f i n}$-morfismo bijetor:

Como o diagrama abaixo comuta e $p_{\Delta}: G \rightarrow G / \Delta$ é $\mathbf{R S G}^{\text {Haus }}$-morfismo sobrejetor temos que $\mathfrak{g}_{\Delta}: \mathcal{P}(G) \rightarrow G / \Delta$ é $\mathbf{R S G}_{p f}$-morfismo sobrejetor e portanto $\widehat{\mathfrak{g}_{\Delta}}: \mathcal{P}(G) / k e r\left(\mathfrak{g}_{\Delta}\right) \mapsto G / \Delta$ é $\mathbf{R S G}_{f i n}$-morfismo bijetor.

\footnotetext{
${ }^{50} \operatorname{Em} 5.54 .(\mathrm{b}): g: \mathcal{P}(G / \Sigma) \longrightarrow \mathcal{P}(G) / \bar{\Sigma} ;$ nesta secão, em $5.68: \overline{\mathcal{P}}\left(p_{\Sigma}\right): \mathcal{P}(G) / \operatorname{ker}\left(\mathcal{P}\left(p_{\Sigma}\right)\right) \longrightarrow \mathcal{P}(G / \Sigma)$ Segue da comutativade dos diagramas envolvidos em ambos os casos que as flechas referidas são inversas uma da outra e que $\operatorname{ker}\left(\mathcal{P}\left(p_{\Sigma}\right)\right)=\bar{\Sigma}$.
} 


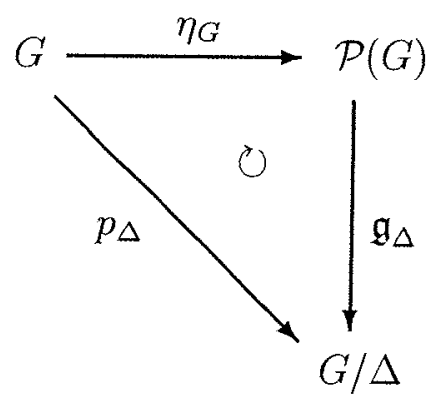

* $\widehat{\mathfrak{g}_{\Delta}}: \mathcal{P}(G) / \operatorname{ker}\left(\mathfrak{g}_{\Delta}\right) \rightarrow G / \Delta$ é $\mathbf{R S G}_{f i n}$-retração:

Como $\Delta=\operatorname{ker}\left(p_{\Delta}\right)=\operatorname{ker}\left(\mathfrak{g}_{\Delta} \circ \eta_{G}\right)=\eta_{G}^{*}\left(\operatorname{ker}\left(\mathfrak{g}_{\Delta}\right)\right)$ segue da definição de morfismo derivado que o diagrama abaixo comuta:

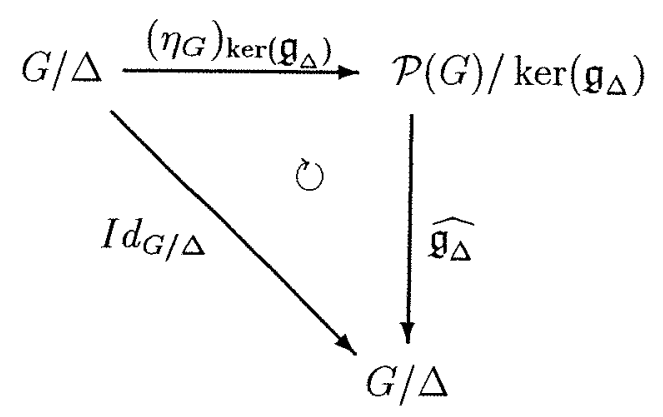

* $\widehat{\mathfrak{g}_{\Delta}}: \mathcal{P}(G) / \operatorname{ker}\left(\mathfrak{g}_{\Delta}\right) \stackrel{\cong}{\cong} G / \Delta$ é $\mathbf{R S G}_{f i n}$-isomorfismo:

Mostramos que o $\mathbf{R S G}_{f i n}$-morfismo bijetor $\widehat{\mathfrak{g}_{\Delta}}: \mathcal{P}(G) / k \operatorname{ker}\left(\mathfrak{g}_{\Delta}\right) \nrightarrow G / \Delta$ admite $\mathbf{R S G}_{f i n}$-seção $\left(\eta_{G}\right)_{k e r\left(\mathfrak{g}_{\Delta}\right)}: G / \Delta \mapsto \mathcal{P}(G) / k e r\left(\mathfrak{g}_{\Delta}\right)$ e portanto esses são $\mathbf{R S G}_{f i n}$-isomorfismos inversos.

(ii) Na notação vigente neste item temos:

Para cada $h=\left(g_{\Delta} / \Delta\right)_{\Delta \in \mathcal{V}_{(G)}} \in \mathcal{P}(G)$ temos:

$$
\begin{aligned}
\widehat{\mathfrak{g}_{\Sigma}} \circ p_{\Delta^{\prime}, \Sigma^{\prime}}\left(h / \Delta^{\prime}\right) & =\widehat{\mathfrak{g}_{\Sigma}}\left(h / \Sigma^{\prime}\right)=g_{\Sigma} / \Sigma= \\
p_{\Delta, \Sigma}\left(g_{\Delta} / \Delta\right) & =p_{\Delta, \Sigma} \circ \widehat{\mathfrak{g}_{\Delta}}\left(h / \Delta^{\prime}\right) .
\end{aligned}
$$

(iii)

Segue diretamente de (i) e (ii).

(iv)

Pelas proposições 5.25 e 5.26.(b) e o item (i) acima.

Lema 5.67. Para $G \in \mathbf{R S G}^{\text {Haus }}$ e cada $\Theta \subseteq G$ subgrupo saturado e fechado temos:

(i) Temos bijeções inversas, crescentes e naturais em $\Theta \in \operatorname{Ssat}(G) \cap \operatorname{Closed}(G)$ :

* $\left(p_{\Theta}\right)^{*} \uparrow: \mathcal{V}(G / \Theta) \stackrel{\cong}{\rightarrow} \mathcal{V}_{\Theta}(G): \Gamma \in \mathcal{V}(G / \Theta) \mapsto\left(p_{\Theta}\right)^{*}(\Gamma) \in \mathcal{V}_{\Theta}(G) ;$

* $\left(p_{\Theta}\right)_{*}\left\lceil: \mathcal{V}_{\Theta}(G) \stackrel{\cong}{\rightrightarrows} \mathcal{V}(G / \Theta): \Delta \in \mathcal{V}_{\Theta}(G) \mapsto\left(p_{\Theta}\right)_{*}(\Delta)=\Delta / \Theta \in \mathcal{V}_{\Theta}(G)\right.$. 
(ii) Para cada $\Delta \in \mathcal{V}_{\Theta}(G)$ o $\mathbf{R S G}^{\text {Haus }}$-morfismo sobrejetor $p_{\Theta, \Delta}: G / \Theta \rightarrow G / \Delta$

é tal que:

$e ́ \mathbf{R S G}_{\text {fin }}$-isomorfismo.

$$
\overline{p_{\Theta, \Delta}}:(G / \Theta) /(\Delta / \Theta) \stackrel{\cong}{\longmapsto} G / \Delta
$$

(iii) $\mathcal{P}\left(p_{\Theta}\right): \lim G / \Delta \rightarrow \lim (G / \Theta) / \Gamma$ é o $\mathbf{R S G}_{p f}$-morfismo sobrejetor dado por:

$$
\begin{aligned}
& \Delta \in \overleftarrow{\mathcal{V}}_{(G)} \quad \Gamma \in \overleftarrow{\mathcal{V}}_{(G / \Theta)} \\
& \left(g_{\Delta} / \Delta\right)_{\Delta \in \mathcal{V}(G)} \in \mathcal{P}(G) \mapsto \\
& \left(\left(g_{\Delta} / \Theta\right) /(\Delta / \Theta)\right)_{\Delta / \Theta \in \mathcal{V}(G / \Theta)} \in \mathcal{P}(G / \Theta)
\end{aligned}
$$

(iv) Existe um único $\mathbf{R S G}_{p f}$-isomorfismo:

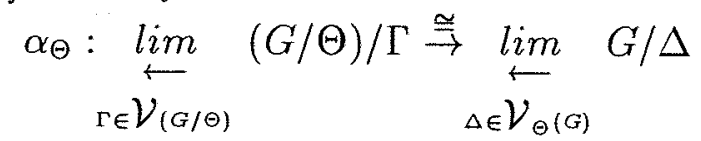

tal que, para cada $\Delta \in \mathcal{V}_{\Theta}(G)$, o diagrama abaixo comuta:

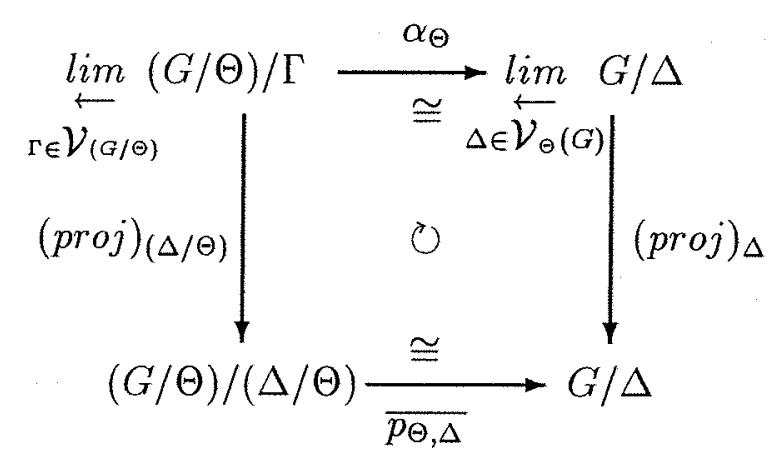

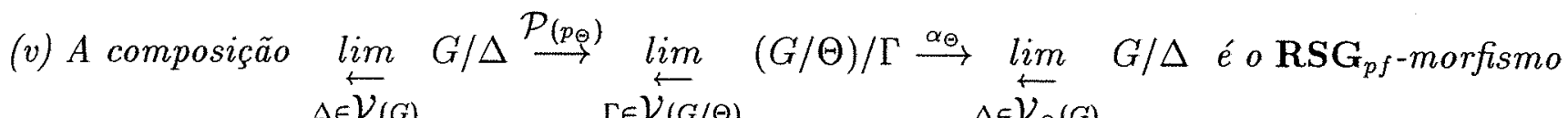
projeção:

$$
\Delta \in \overleftarrow{\mathcal{V}}_{(G)} \quad \Gamma \in \overleftarrow{\mathcal{V}}(G / \Theta)^{\leftarrow} \overleftarrow{\mathcal{V}}_{\Theta}(G)
$$

$$
\begin{gathered}
\rho_{\Theta}: \lim _{\Delta \in \mathcal{V}_{(G)}} G / \Delta \longrightarrow \underset{\Delta \in \overleftarrow{\mathcal{V}}_{\Theta}(G)}{\lim } G / \Delta \\
\left(g_{\Delta} / \Delta\right)_{\Delta \in \mathcal{V}(G)}
\end{gathered}
$$

\section{Prova.}

O item (i) e (ii) estão 5.58. O item (iii) decorre de (i) e da definição do funtor $\mathcal{P}$. O item (iv) segue diretamente de (i) e (ii) anteriores e o item (v) segue de (iii) e (iv).

Teorema 5.68. O funtor $\mathcal{P}: \mathbf{R S G}^{\text {Haus }} \longrightarrow \mathbf{R S G}_{p f}$ preserva quocientes por subgrupos saturados e fechados i.e. :

Se $f: G \rightarrow H$ é $\mathbf{R S G}^{\text {Haus }}$-morfismo sobrejetor tal que $\bar{f}: G / k e r(f) \stackrel{\cong}{\rightsquigarrow} H$ é $\mathbf{R S G}^{\text {Haus }}$ isomorfismo então $\mathcal{P}(f)$ é $\mathbf{R S G}_{p f}$-morfismo sobrejetor tal que $\overline{\mathcal{P}(f)}: \mathcal{P}(G) / k e r(\mathcal{P}(f)) \stackrel{\cong}{\cong}$ $\mathcal{P}(H)$ é $\mathbf{R S G}_{p f}$-isomorfismo. 

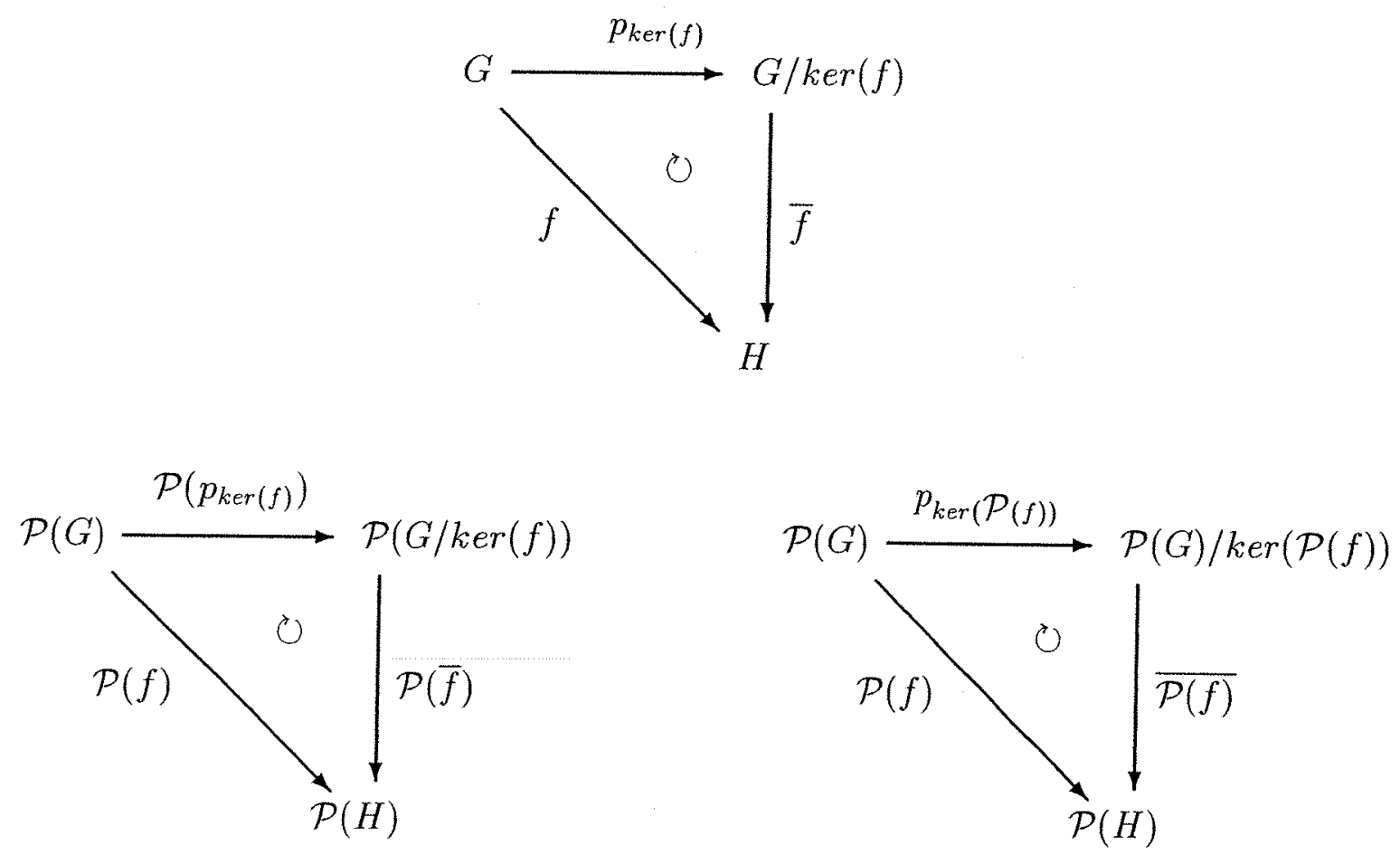

\section{Prova.}

Observação: Como $f: G \rightarrow H$ é RSG ${ }^{\text {Haus }}$-morfismo sobrejetor temos, pela proposição 5.65.(i), que a flecha obtida de $\mathcal{P}(f)$ por passagem ao quociente: $\overline{\mathcal{P}(f)}: \mathcal{P}(G) / \operatorname{ker}(\mathcal{P}(f)) \mapsto \mathcal{P}(H)$ é um RSG-morfismo bijetor que é homeomorfismo de grupos topológicos booleanos onde $\operatorname{ker}(\mathcal{P}(f)) \subseteq \mathcal{P}(G)$ é um subgrupo saturado e fechado. Portanto para provarmos que $\overline{\mathcal{P}(f)}$ é um $\mathbf{R S G}_{p f}$-isomorfismo bastaria que verificássemos que $\overline{\mathcal{P}(f)}$ é um $L_{S G}$-monomorfismo.

A seguir reduziremos nosso problema e, fazendo identificações a partir dos lemas acima e trabalhando com a topologia em $\mathcal{P}(G)$, atingimos nosso objetivo:

(I) Redução: Basta verificarmos que para cada $G \in \mathbf{R S G}^{\text {Haus }}$ e cada $\Theta \subseteq G$ subgrupo saturado e fechado temos que:

$p_{\Theta}: G \rightarrow G / \Theta$ é $\mathbf{R S G}^{\text {Haus }}$-morfismo sobrejetor tal que $\operatorname{ker}\left(p_{\Theta}\right)=\Theta$ e $\overline{p_{\Theta}}=I d_{G / \Theta}: G / \Theta \rightarrow$ $G / \Theta \Rightarrow$

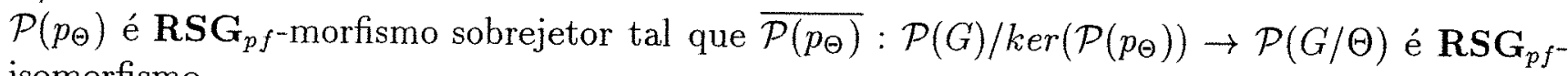
isomorfismo.

Prova de (I)

Para cada $G, H \in \mathbf{R S G}^{\text {Haus }}$ e cada $R S G$-morfismo $f: G \rightarrow H$ temos o diagrama comutativo na categoria $\mathbf{R S G}^{\text {Haus }}$ : 
Onde:

* $G_{f} \doteq G / k e r(f)$;

* $\widetilde{f} \doteq p_{k e r(f)}$;

* $\Sigma_{f} \doteq \operatorname{ker}(\mathcal{P}(\tilde{f}))$;

$* \Gamma_{f} \doteq k e r(\mathcal{P}(f))$.

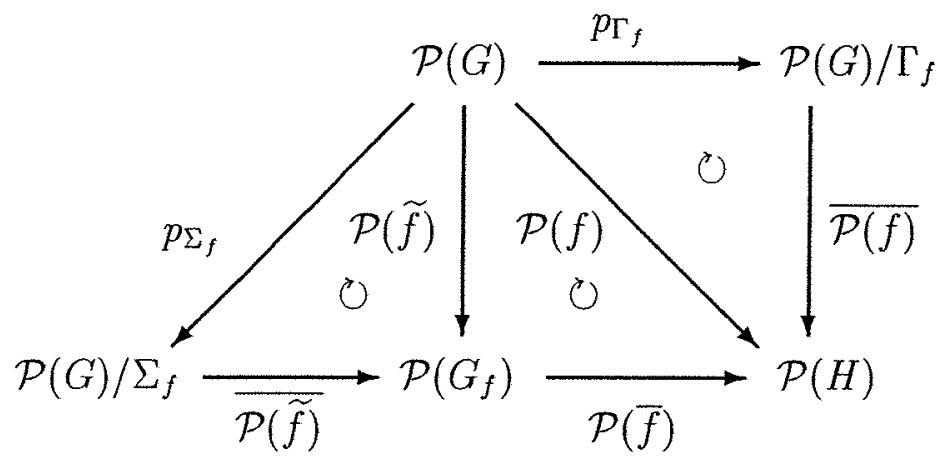

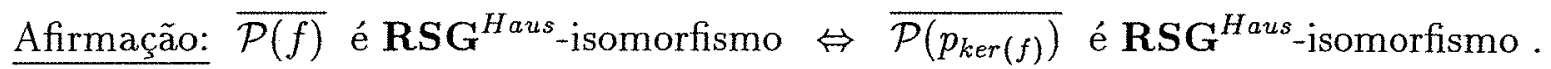

(1) Como $\bar{f}: G / k e r(f) \stackrel{\cong}{\rightsquigarrow} H$ é $R S G$-isomorfismo temos:

* $\mathcal{P}(\bar{f})$ é $R S G$-isomorfismo : pois $\mathcal{P}$ é funtor.

* $\Sigma_{f}=\operatorname{ker}\left(\mathcal{P}\left(p_{k e r(f)}\right)\right)=\operatorname{ker}(\mathcal{P}(f))=\Gamma_{f}:$

Pois, pelos diagramas comutativos abaixo do enunciado do teorema, temos:

Pela definição de $\bar{f}$ temos

$$
f=\bar{f} \circ p_{k e r(f)}
$$

Como $\mathcal{P}$ é funtor segue que,

$$
\mathcal{P}(f)=\mathcal{P}(\bar{f}) \circ \mathcal{P}\left(p_{k e r(f)}\right)
$$

Logo:

$$
\operatorname{ker}(\mathcal{P}(f))=\left(\mathcal{P}\left(p_{k e r(f)}\right)\right)^{*}(k \operatorname{er}(\mathcal{P}(\bar{f}))) .
$$

Como $\mathcal{P}(\bar{f})$ é $\mathbf{R S G}_{p f^{-}}$-isomorfismo temos, em particular,

$$
\operatorname{ker}(\mathcal{P}(\bar{f}))=\left\{1_{\mathcal{P}\left(G_{f}\right)}\right\}
$$

Logo:

$$
\operatorname{ker}(\mathcal{P}(f))=\left(\mathcal{P}\left(p_{k e r(f)}\right)\right)^{*}\left(\left\{1_{\mathcal{P}_{\left(G_{f}\right)}}\right\}\right)=\operatorname{ker}\left(\mathcal{P}\left(p_{k e r(f)}\right)\right)
$$

(2) $\overline{\mathcal{P}(f)}=\mathcal{P}(\bar{f}) \circ \overline{\mathcal{P}\left(p_{k e r(f)}\right)}$ :

* Como o diagrama acima comuta temos:

$$
\overline{\mathcal{P}(f)} \circ p_{\Gamma_{f}}=\left(\mathcal{P}(\bar{f}) \circ \overline{\mathcal{P}\left(p_{k e r(f)}\right)}\right) \circ p_{\Sigma_{f}} .
$$

* Como $\Gamma_{f}=\Sigma_{f}$ temos que $p_{\Gamma_{f}}=p_{\Sigma_{f}}$ são o mesmo $R S G$-morfismo sobrejetor e, como um $R S G$-morfismo sobrejetor é cancelável à direita, temos que a afirmação segue.

$$
\overline{\mathcal{P}(f)} \text { é } \mathbf{R S G}^{\text {Haus }} \text {-isomorfismo } \Leftrightarrow \overline{\mathcal{P}\left(p_{\text {ker }(f)}\right)} \text { é } \mathbf{R S G}^{\text {Haus }} \text {-isomorfismo . }
$$

Pois vimos acima que $\mathcal{P}(\bar{f})$ é $\mathbf{R S G}_{p f}$-isomorfismo e que $\overline{\mathcal{P}(f)}=\mathcal{P}(\bar{f}) \circ \overline{\mathcal{P}\left(p_{k e r(f)}\right)}$. 
(II) Para cada $G \in \mathbf{R S G}^{\text {Haus }}$ e cada $\Theta \in S$ sat $(G) \cap C l o s e d(G)$ temos que $\mathcal{P}\left(p_{\Theta}\right)$ é $\mathbf{R S G}_{p f^{-}}$ morfismo sobrejetor tal que $\overline{\mathcal{P}\left(p_{\Theta}\right)}: \mathcal{P}(G) / \operatorname{ker}\left(\mathcal{P}\left(p_{\Theta}\right)\right) \rightarrow \mathcal{P}(G / \Theta)$ é $\mathbf{R S G}_{p f}$-isomorfismo:

(1) Estudando $\operatorname{ker}\left(\mathcal{P}\left(p_{\Theta}\right)\right)$

Vimos em 5.65.(i) que $\overline{\mathcal{P}\left(p_{\Theta}\right)}: \mathcal{P}(G) / \Sigma_{\Theta} \rightarrow \mathcal{P}(G / \Theta): h / \Sigma_{\Theta} \mapsto \mathcal{P}\left(p_{\Theta}\right)(h)$, onde $\Sigma_{\Theta} \doteq$ $\operatorname{ker}\left(\mathcal{P}\left(p_{\Theta}\right)\right) \subseteq \mathcal{P}(G)$ é subgrupo saturado fechado, é um RSG-morfismo bijetor que é um isomorfismo de grupos topológicos booleanos.

Abaixo estudaremos a aplicação :

$$
\Theta \in \operatorname{Ssat}(G) \cap C \text { losed }(G) \mapsto \Sigma_{\Theta} \in \operatorname{Ssat}(\mathcal{P}(G)) \cap C l o s e d(\mathcal{P}(G))
$$

(a) $\Sigma_{\Theta}=\bigcap\left\{k e r\left(\mathfrak{g}_{\Delta}\right): \Delta \in \mathcal{V}_{\Theta}(G)\right\}$ :

Por definição temos: $\Sigma_{\Theta} \doteq \operatorname{ker}\left(\mathcal{P}\left(p_{\Theta}\right)\right)$;

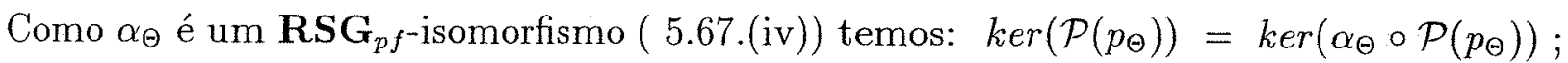

Por 5.67.(v) temos: $\operatorname{ker}\left(\alpha_{\Theta} \circ \mathcal{P}\left(p_{\Theta}\right)\right)=\operatorname{ker}\left(\rho_{\Theta}\right)$;

Por definição de $\rho_{\Theta}$ temos:

$\operatorname{ker}\left(\rho_{\Theta}\right)=\left\{\left(g_{\Delta} / \Delta\right)_{\Delta \in \mathcal{V}_{(G)}} \in \mathcal{P}(G):\left(g_{\Delta} / \Delta\right)_{\Delta \in \mathcal{V}_{\Theta}(G)}=(1 / \Delta)_{\Delta \in \mathcal{V}_{\Theta}(G)}\right\}$

Assim: $\operatorname{ker}\left(\rho_{\Theta}\right)=\left\{\left(g_{\Delta} / \Delta\right)_{\Delta \in \mathcal{V}(G)} \in \mathcal{P}(G): \forall \Delta \in \mathcal{V}_{\Theta}(G)\left(g_{\Delta} / \Delta=1 / \Delta \in G / \Delta\right)\right\}$;

Pela definição de $\mathfrak{g}_{\Delta}: \mathcal{P}(G) \rightarrow G / \Delta, \Delta \in \mathcal{V}(G)$ temos:

$\Sigma_{\Theta}=\operatorname{ker}\left(\rho_{\Theta}\right)=\left\{h \in \mathcal{P}(G): \forall \Delta \in \mathcal{V}_{\Theta}(G)\left(\mathfrak{g}_{\Delta}(h)=1 / \Delta \in G / \Delta\right)\right\} ;$

Logo: $\Sigma_{\Theta}=\operatorname{ker}\left(\rho_{\Theta}\right)=\bigcap\left\{k \operatorname{ker}\left(\mathfrak{g}_{\Delta}\right): \Delta \in \mathcal{V}_{\Theta}(G)\right\}$.

(b) $\Theta \in \mathcal{V}(G) \Rightarrow \Sigma_{\Theta}=\operatorname{ker}\left(\mathfrak{g}_{\Theta}\right) \in \mathcal{V}(\mathcal{P}(G))$ :

Pois se $\Theta \in \mathcal{V}(G)$ então $\mathcal{V}_{\Theta}(G)$ admite $\Theta$ como menor elemento e, para cada $\Delta \in \mathcal{V}_{\Theta}(G)$ temos $\operatorname{ker}\left(\mathfrak{g}_{\Theta}\right) \subseteq \operatorname{ker}\left(\mathfrak{g}_{\Delta}\right)$. Logo: $\quad k e r\left(\mathfrak{g}_{\Theta}\right) \subseteq \bigcap\left\{k e r\left(\mathfrak{g}_{\Delta}\right): \Delta \in \mathcal{V}_{\Theta}(G)\right\} \subseteq \operatorname{ker}\left(\mathfrak{g}_{\Theta}\right)$.

Portanto:

$$
\operatorname{ker}\left(\mathfrak{g}_{\Theta}\right)=\bigcap\left\{k e r\left(\mathfrak{g}_{\Delta}\right): \Delta \in \mathcal{V}_{\Theta}(G)\right\} \underset{(a)}{=} \Sigma_{\Theta}
$$

(c) Temos função crescente: $\left.\left(\Delta \subseteq \Delta^{\prime}\right) \in \mathcal{V}_{\Theta}(G) \mapsto\left(\Sigma_{\Delta} \subseteq \Sigma_{\Delta^{\prime}}\right) \in \mathcal{V}_{\Sigma_{\Theta}}(\mathcal{P}(G))\right)$ :

* $\Delta \in \mathcal{V}_{\Theta}(G) \Rightarrow \Sigma_{\Delta} \in \mathcal{V}_{\Sigma_{\Theta}}(\mathcal{P}(G))$ :

Para cada $\Delta \in \mathcal{V}_{\Theta}(G)$ temos $\Sigma_{\Delta}=\operatorname{ker}\left(\mathfrak{g}_{\Delta}\right)$. Como $\operatorname{ker}\left(\mathfrak{g}_{\Delta}\right) \in \mathcal{V}(\mathcal{P}(G))$ e $\Sigma_{\Theta}=\bigcap\left\{k e r\left(\mathfrak{g}_{\Delta}\right)\right.$ : $\left.\Delta \in \mathcal{V}_{\Theta}(G)\right\}$, o resultado segue.

* $\left(\Delta \subseteq \Delta^{\prime}\right) \in \mathcal{V}_{\Theta}(G) \Rightarrow\left(\Sigma_{\Delta} \subseteq \Sigma_{\Delta^{\prime}}\right)$ :

Pois: $\quad \Sigma_{\Delta}=\operatorname{ker}\left(\mathfrak{g}_{\Delta}\right) \subseteq \operatorname{ker}\left(p_{\Delta, \Delta^{\prime}} \circ \mathfrak{g}_{\Delta}\right)=\operatorname{ker}\left(\mathfrak{g}_{\Delta^{\prime}}\right)=\Sigma_{\Delta^{\prime}}$.

(d) $\left\{\Sigma_{\Delta}: \Delta \in \mathcal{V}_{\Theta}(G)\right\} \subseteq \mathcal{V}_{\Sigma_{\Theta}}(\mathcal{P}(G))$ é um conjunto ordenado dirigido para baixo tal que $\Sigma_{\Theta}=\bigcap\left\{\Sigma_{\Delta}: \Delta \in \mathcal{V}_{\Theta}(G)\right\}$ :

Os tópicos (b) e (c) garantem que $\left\{\Sigma_{\Delta}: \Delta \in \mathcal{V}_{\Theta}(G)\right\} \subseteq \mathcal{V}_{\Sigma_{\Theta}}(\mathcal{P}(G))$ é um conjunto ordenado dirigido para baixo.

Pelos tópicos (a) e (b) temos:

$$
\Sigma_{\Theta}=\bigcap\left\{k e r\left(\mathfrak{g}_{\Delta}\right): \Delta \in \mathcal{V}_{\Theta}(G)\right\}=\bigcap\left\{\Sigma_{\Delta}: \Delta \in \mathcal{V}_{\Theta}(G)\right\}
$$


(e) Observamos que se $\Theta \in \mathcal{V}(G)$ então $\overline{\mathcal{P}\left(p_{\Theta}\right)}: \mathcal{P}(G) / \Sigma_{\Theta} \stackrel{\cong}{\cong}(G / \Theta)$ :

Pois, neste caso, temos:

* $\eta_{G / \Theta}: G / \Theta \stackrel{\cong}{\cong} \mathcal{P}(G / \Theta):$ pois $G / \Theta$ é finito.

* $\widehat{\mathfrak{g}_{\Theta}}: \mathcal{P}(G) / k e r\left(\mathfrak{g}_{\Theta}\right) \stackrel{\cong}{\nrightarrow} G / \Theta:$ pelo lema 5.66 .

* $\operatorname{ker}\left(\mathfrak{g}_{\Theta}\right)=\Sigma_{\Theta}:$ como vimos acima.

* $\overline{\mathcal{P}\left(p_{\Theta}\right)}=\eta_{G / \Theta} \circ \widehat{\mathfrak{g}_{\Theta}}$ : após alguma "caça ao diagrama"!

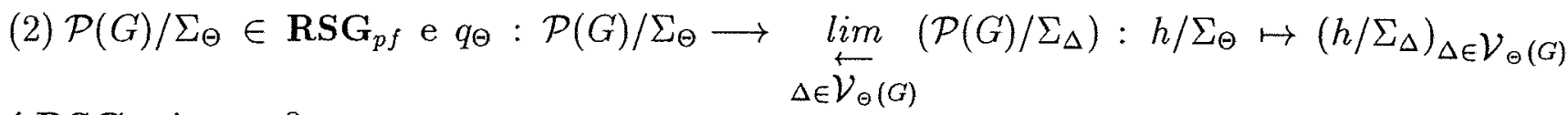
é $\mathbf{R S G}_{p f}$-isomorfismo:

Vimos no tópico (1) acima que $\left\{\Sigma_{\Delta}: \Delta \in \mathcal{V}_{\Theta}(G)\right\} \subseteq \mathcal{V}_{\Sigma_{\Theta}}(\mathcal{P}(G))$ é um conjunto ordenado dirigido para baixo tal que $\Sigma_{\Theta}=\bigcap\left\{\Sigma_{\Delta}: \Delta \in \mathcal{V}_{\Theta}(G)\right\}$. Assim $\Sigma_{\Theta} \subseteq S \operatorname{sat}(\mathcal{P}(G)) \cap C$ losed $(\mathcal{P}(G))$ possui a propriedade de separação topológica ([pst]: veja Observação 5.31.(ii)) e, pelo teorema 5.28 temos que $\mathcal{P}(G) / \Sigma_{\Theta} \in \mathbf{R S G}_{p f}$. Segue da Observação 5.31.(iii) que $\left\{\Sigma_{\Delta} / \Sigma_{\Theta}: \Delta \in \mathcal{V}_{\Theta}(G)\right\}$ é um pf-sistema em $\mathcal{P}(G) / \Sigma_{\Theta}$ e que $q_{\Theta}: \mathcal{P}(G) / \Sigma_{\Theta} \cong \lim \mathcal{P}(G) / \Sigma_{\Delta}$ é RSG $\mathbf{R f}_{\text {-isomorfismo. }}$ $\Delta \in \overleftarrow{V}_{\Theta}(G)$

(3) Temos diagrama comutativo:

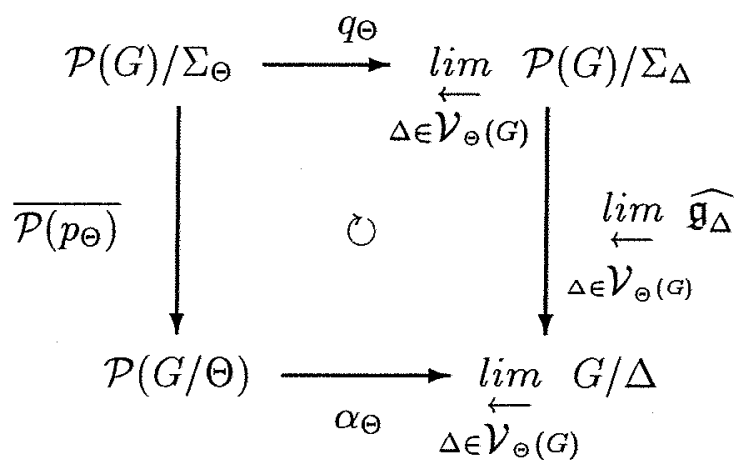

Onde:

* $\lim _{\Delta \in \overleftarrow{V}_{\Theta}(G)} \widehat{\mathfrak{g}_{\Delta}}: \lim _{\Delta \in \overleftarrow{V}_{\Theta}(G)}(\mathcal{P}(G)) / \operatorname{ker}\left(\mathbf{g}_{\Delta}\right) \longrightarrow \underset{\Delta \in \overleftarrow{V}_{\Theta}(G)}{\lim } G / \Delta$ é o RSG $\mathbf{R S}^{\text {Haus }}$-morfismo definido no lema 5.66.(iii) .

* $\alpha_{\Theta}: \lim (G / \Theta) / \Gamma \longrightarrow \lim G / \Delta$ é o $\mathbf{R S G}^{\text {Haus }}$-morfismo definido no lema 5.67.(iv). $\Gamma \in \mathcal{V}_{(G / \Theta)} \quad \Delta \in \mathcal{V}_{\Theta(G)}$

* $\overline{\mathcal{P}\left(p_{\Theta}\right)}: \mathcal{P}(G) / \Sigma_{\Theta} \longrightarrow \mathcal{P}(G / \Theta)$ é o $\mathbf{R S G}^{\text {Haus }}$-morfismo definido no tópico (1) acima.

* $q_{\Theta}: \mathcal{P}(G) / \Sigma_{\Theta} \longrightarrow \lim _{\leftarrow}\left(\mathcal{P}(G) / \Sigma_{\Delta}\right)$ é o $\mathbf{R S G}^{\text {Haus }}$-morfismo definido no tópico (2) acima. $\Delta \in \mathcal{V}_{\Theta}(G)$ 
Verificaremos agora que o diagrama, de fato, comuta:

Para cada $h=\left(g_{\Delta} / \Delta\right)_{\Delta \in \mathcal{V}(G)} \in \mathcal{P}(G)$ temos:

Pela definição de $\overline{\mathcal{P}\left(p_{\Theta}\right)}$ temos:

$\alpha_{\Theta} \circ \overline{\mathcal{P}\left(p_{\Theta}\right)}\left(h / \Sigma_{\Theta}\right)=\alpha_{\Theta} \circ \mathcal{P}\left(p_{\Theta}\right)(h) ;$

Por 5.67.(v) temos: $\alpha_{\Theta} \circ \mathcal{P}\left(p_{\Theta}\right)(h)=\rho_{\Theta}(h)$;

Pela definição de $\rho_{\Theta}$ em 5.67.(v) : $\rho_{\Theta}(h)=\rho_{\Theta}\left(\left(g_{\Delta} / \Delta\right)_{\Delta \in \mathcal{V}(G)}\right)=\left(g_{\Delta} / \Delta\right)_{\Delta \in \mathcal{V}_{\Theta}(G)}$;

Pela definição dos morfismos $\mathfrak{g}_{\Delta}$ e $\widehat{\mathfrak{g}_{\Delta}}, \Delta \in \mathcal{V}(G)$ segue que: $\widehat{\mathfrak{g}_{\Delta}}\left(h / k e r\left(\mathfrak{g}_{\Delta}\right)\right)=\mathfrak{g}_{\Delta}(h)=g_{\Delta} / \Delta$;

Mas vimos no tópico (1).(b) que para cada $\Delta \in \mathcal{V}(G)$ temos: $\Sigma_{\Delta}=\operatorname{ker}\left(\mathfrak{g}_{\Delta}\right)$;

Segue que: $\quad \rho_{\Theta}(h)=\left(g_{\Delta} / \Delta\right)_{\Delta \in \mathcal{V}_{\Theta}(G)}=\left(\widehat{\mathfrak{g}_{\Delta}}\left(h / \Sigma_{\Delta}\right)\right)_{\Delta \in \mathcal{V}_{\Theta}(G)} ;$

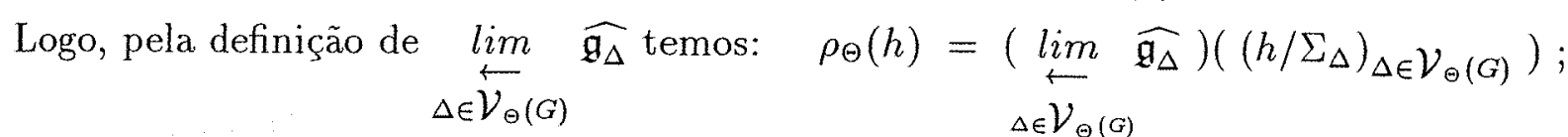

Finalmente, como $\left.q_{\Theta}\left(h / \Sigma_{\Theta}\right)=\left(\left(h / \Sigma_{\Delta}\right)\right)_{\Delta \in \mathcal{V}_{\Theta}(G)}\right)$, obtemos:

$$
\begin{aligned}
& \alpha_{\Theta} \circ \overline{\mathcal{P}\left(p_{\Theta}\right)}\left(h / \Sigma_{\Theta}\right)=\rho_{\Theta}(h)=\left(\lim _{\longleftarrow} \widehat{\mathfrak{g}_{\Delta}}\right) \circ q_{\Theta}\left(h / \Sigma_{\Theta}\right) . \\
& \Delta \in \mathcal{V}_{\Theta(G)}
\end{aligned}
$$

(4) $\overline{\mathcal{P}\left(p_{\Theta}\right)}: \mathcal{P}(G) / \Sigma_{\Theta} \longrightarrow \mathcal{P}(G / \Theta)$ é $\mathbf{R S G}_{p f^{-}}$isomorfismo.

Pois:

* $\lim _{\Delta \in \mathcal{V}_{\Theta}(G)} \widehat{\mathfrak{g}_{\Delta}}: \lim _{\Delta \in \overleftarrow{V}_{\Theta}(G)}(\mathcal{P}(G)) / \operatorname{ker}\left(\mathfrak{g}_{\Delta}\right) \stackrel{\cong}{\longrightarrow} \underset{\Delta \in \overleftarrow{V}_{\Theta}(G)}{\lim } G / \Delta$ é $\mathbf{R S G}_{p f}$-isomorfismo:

Pelo lema 5.66.(iii) .

* $\alpha_{\Theta}: \lim _{\leftarrow}(G / \Theta) / \Gamma \stackrel{\cong}{\longrightarrow} \lim G / \Delta$ é $\mathbf{R S G}_{p f}$-isomorfismo:

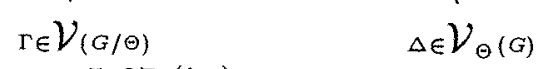

Pelo lema 5.67.(iv).

* $q_{\Theta}: \mathcal{P}(G) / \Sigma_{\Theta} \cong \lim _{\leftarrow}\left(\mathcal{P}(G) / \Sigma_{\Delta}\right)$ é $\mathbf{R S G}_{p f}$-isomorfismo:

Pelo tópico (2) acima.

$$
\Delta \in \overleftarrow{\mathcal{V}}_{\Theta}(G)
$$

* $\alpha_{\Theta} \circ \overline{\mathcal{P}\left(p_{\Theta}\right)}=\left(\stackrel{\lim }{\longleftarrow} \widehat{\mathfrak{g}_{\Delta}}\right) \circ q_{\Theta}:$

Pelo tópico (3) acima.

$$
\Delta \in \mathcal{V}_{\Theta(G)}
$$

Observação 5.69. O teorema acima aplicado em objetos $G \in \mathbf{R S G}_{p f}$ e a algum $\Theta \in S \operatorname{sat}(G) \cap$

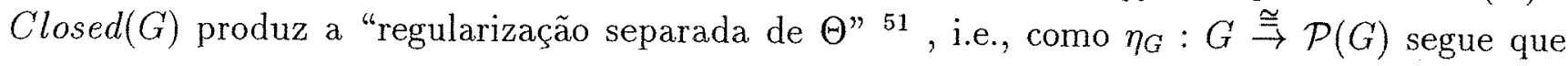

\footnotetext{
${ }^{51}$ Veja a Observação 5.31.(iv).
} 
$\widehat{\Theta} \doteq\left(\eta_{G}\right)^{-1}\left[\Sigma_{\Theta}\right]$ é o menor subgrupo saturado e fechado de $G$ que satisfaz [pst] e que contém $\Theta$. Pois, de fato, temos $\widehat{\Theta}=\bigcap\left\{\Delta \in \mathcal{V}_{\Theta}(G)\right\}$. 


\section{Capítulo 6}

\section{O funtor $\mathcal{P}$, Álgebras de Boole e Aplicações}

\subsection{Introdução}

Na subseção 3.4.3 do Capítulo 3 apresentamos algumas das relações fundamentais entre álgebras de Boole e grupos especiais reduzidos. A tabela baixo resume algumas das correspondências de conceitos no caso geral e no caso das álgebras de Boole.

\begin{tabular}{|c|c|}
\hline Grupos Especiais & Álgebras de Boole \\
\hline$\cdot$ & $\Delta$ \\
\hline 1 & $\perp$ \\
\hline-1 & $T$ \\
\hline$a \in D_{G}(1, b)$ & $a \leq b$ \\
\hline Subgrupo saturado & Ideal \\
\hline SG-morfismo & Ba-morfismo \\
\hline
\end{tabular}

Nesta Introdução relembraremos mais algumas construções acerca de álgebras de Boole, algumas das quais admitiremos conhecidas pelo leitor.

Seja $A$ um álgebra de Boole. Para $S \subseteq A$, denotamos

$$
-S=\{-s: s \in S\}
$$

O resultado que segue é bem conhecido e omitiremos sua prova:

Fato 6.1. Se $A$ é uma álgebra de Boole a aplicação

$$
S \in 2^{A} \longrightarrow-S \in 2^{A}
$$

estabelece uma correspondência natural, biunívoca e crescente entre filtros e ideais em A. Além disso:

a) Esta correpondência fornece uma bijeção natural entre ultrafiltros e ideais maximais em $A$. 
b) Se $\mathcal{U}$ é um ultrafiltro em $A$ então $A=\mathcal{U} \cup-\mathcal{U} .^{1}$

Observação 6.2. O grupo especial $\mathbb{Z}_{2}$ apresentado em 3.13 pode ser tratado como uma álgebra de Boole de dois elementos, $2=\{0,1\}$. Se introduzimos em $\mathbb{Z}_{2}$ uma ordem, declarando

$$
1<-1
$$

então a aplicação $\alpha: \mathbb{Z}_{2} \longrightarrow 2$ tal que $\alpha(1)=0$ e $\alpha(-1)=1$ :

* É um isomorfismo entre a estrutura de grupo multiplicativo em $\mathbb{Z}_{2}$ e a estrutura $\langle 2, \Delta\rangle$, onde $\triangle$ é a diferença simétrica em 2 , isto é, a soma módulo 2 ;

* É um isomorfismo entre a estrutura de ordem definida em $\mathbb{Z}_{2}$ e aquela em 2 ;

* É um isomorfismo entre a estrutura de grupo especial reduzido, descrita em 3.13 , e a de grupo especial reduzido na álgebra booleana 2 , descrita na subseção 3.4 .3 , isto é, para $a, b, c, d \in \mathbb{Z}_{2}$

$$
\langle a, b\rangle \equiv_{\mathbb{Z}_{2}}\langle c, d\rangle \Leftrightarrow a+b=c+d \Leftrightarrow\left\{\begin{array}{l}
\alpha(a) \vee \alpha(b)=\alpha(c) \vee \alpha(d) ; \\
\alpha(a) \wedge \alpha(b)=\alpha(c) \wedge \alpha(d) .
\end{array}\right.
$$

Daqui em diante, consideraremos, quando necesário, o grupo especial $\mathbb{Z}_{2}$ como idêntico à álgebra de Boole 2, embora mantendo símbolos distintos para indicá-los.

A identificação em 6.2 e o fato que morfismos de álgebras de Boole e SG-morfismos são noções essencialmente equivalentes ${ }^{2}$ (3.47.(b)), têm a seguinte consequência

Lema 6.3. Se $A$ é uma álgebra de Boole e $\sigma: A \longrightarrow \mathbb{Z}_{2}$ é um morfismo de grupos que leva $\top$ $(=-1)$ em -1 em $\mathbb{Z}_{2}$, as seguintes condições são equivalentes :

(1) $\sigma$ é um $S G$-carácter de A;

(2) $\sigma$ é um Ba-morfismo;

(3) ker $\sigma$ é um ideal maximal em A;

(4) coker $\sigma=\{x \in A: \sigma(x)=-1\}$ é um ultrafiltro em $A$.

Prova. A equivalência entre (1) e (2) vem de 3.47.(b). A equivalência entre (2), (3) e (4) são fatos bem conhecidos.

6.4. Como existem correspondências naturais e biunívocas entre
(1) Ultrafiltros em $A$;
(2) Ideais maximais em $A$;
(3) Ba-morfismos de $A$ em 2, então, por 6.3, podemos identificar o espaço de ordens de $A$ com o espaço de Stone de $A$, isto é,

\footnotetext{
${ }^{1}$ Esta união é disjunta, pois ultrafiltros são filtros maximais e próprios.

${ }^{2} \mathrm{Na}$ realidade, interdefíniveis.
} 


$$
X_{A}=\{\mathcal{U} \subseteq A: \mathcal{U} \text { é um ultrafiltro em } A\}
$$

é o espaço de ordens de $A$ como grupo especial. A identificação se dá de modo que :

* Se $\sigma$ é um SG-carácter de $A$, o ponto em $X_{A}$ associado à $\sigma$ é o ultrafiltro - ker $\sigma$;

* Se $\mathcal{U}$ é um ultrafiltro em $A$, então $-\mathcal{U}$ é um ideal maximal em $A$ e o núcleo de um único Ba-morfismo, $\sigma_{\mathcal{U}}: A \longrightarrow \mathbb{Z}_{2}$, definido por

$$
\sigma_{\mathcal{U}}(a)=-1 \quad \Leftrightarrow \quad a \in \mathcal{U} .
$$

Lembramos que o espaço de Stone de $A$ vem acompanhado de uma topologia Hausdorff, compacta e com base de abertos e fechados, dada por

$$
X_{a}=\left\{\mathcal{U} \in X_{A}: a \in \mathcal{U}\right\} .
$$

Tendo em conta a identificação feita acima, vemos que ela também é topológica, pois

$$
\text { Para todo } a \in A, X_{a}=[a=-1]^{3}
$$

estabelecendo a ligação estreita, algébrica e topológica, entre o espaço de ordens de $A$ e seu espaço de Stone.

Uma das construçôes fundamentais da Matemática é a dualidade de Stone, que admitiremos conhecida pelo leitor. Um Ba-morfismo, $f: A \longrightarrow B$, induz uma função contínua

$$
X(f): X_{B} \longrightarrow X_{A}
$$

onde para cada $\mathcal{U}^{\prime} \in X_{B}, X(f)\left(\mathcal{U}^{\prime}\right)=f^{-1}\left(\mathcal{U}^{\prime}\right)$. As associações

$$
A \longmapsto X_{A} \quad \text { e } \quad(A \stackrel{f}{\longrightarrow} B) \longmapsto\left(X_{B} \stackrel{X(f)}{\longrightarrow} X_{A}\right)
$$

constituem um funtor contravariante da categoria Ba na categoria BS dos espaços Booleanos e funções contínuas, denominado funtor de Stone.

Fato 6.5. Sejam $A, B$ álgebras de Boole e $A \stackrel{f}{\longrightarrow} B$ um Ba-morfismo. Com a notação acima, a) Para $a, b \in A$,

(1) $X_{a}$ é um aberto e fechado compacto em $X_{A}$. Além disso, $X_{\perp}=\emptyset$ e $X_{\mathrm{T}}=X_{A}$.

(2) Valem as seguintes relações :

$$
X_{a \wedge b}=X_{a} \cap X_{b} ; \quad X_{a \vee b}=X_{a} \cup X_{b} ; \quad X_{-a}=X_{a}^{c} .
$$

Assim, a aplicação $a \in A \longmapsto X_{a} \in 2^{X_{A}}$ é um Ba-isomorfismo entre $A$ e a álgebra de abertos e fechados em $X_{A}$.

b) Para todo $a \in A, \quad X(f)^{-1}\left(X_{a}\right)=X_{f(a)}$ e portanto $X(f)$ é uma função contínua.

c) $f$ é injetor $\Leftrightarrow X(f)$ é uma sobrejeção contínua.

d) $f$ é sobrejetor $\Leftrightarrow X(f)$ é uma injeção contínua.

\footnotetext{
${ }^{3}$ Ver 3.18 .
} 
Em relação aos SG-morfismos de álgebras de Boole em grupos reduzidos, mencionamos o seguinte resultado que é extraído da Proposição 2.3 e da Observação que segue a sua prova em [DM6] :

Proposição 6.6. Seja $G$ um grupo especial reduzido. As seguintes condiçôes são equivalentes:

(1) Todo SG-morfismo injetor de G em um grupo especial reduzido é puro.

(2) G é uma álgebra de Boole.

\subsection{Subgrupos Saturados e Ideais de Índice Finito}

Se $A$ é um conjunto, $C \subseteq_{f} A$ indica que $C$ é um subconjunto finito de $A$.

Se $G$ é um grupo especial reduzido :

* $\chi(G)$ é o grupo dos carácteres de $G$, isto é, o grupo - com multiplicação definida ponto a ponto -, consistindo de todos os morfismos de grupo de $G$ em $\mathbb{Z}_{2}$;

* Para $S \subseteq \chi(G),[S]$ é o subespaço vetorial gerado por $S$ em $\left.\chi_{(} G\right)$.

Como grupos de expoente 2 , tanto $G$ quanto $\chi(G)$ são espaços vetoriais sobre o corpo de dois elementos, $\mathbb{F}_{2}$.

O seguinte resultado será útil no que seguè :

Lema 6.7. Sejam $G$ um grupo especial reduzido, $\tau, \sigma_{1}, \ldots, \sigma_{n} \in X_{G}$ e $\Gamma \subseteq G$ um subgrupo saturado próprio de G. Sejam $\Delta=\bigcap_{i=1}^{n} \operatorname{ker} \sigma_{i}$ e $m \geq 1$ um inteiro.

a) $\tau \in\left[\sigma_{1}, \ldots, \sigma_{n}\right] \Leftrightarrow \Delta \subseteq k e r \tau$.

b) As seguintes condiçôes são equivalentes :

(1) $\left\{\sigma_{1}, \ldots, \sigma_{n}\right\}$ é linearmente independente em $\chi(G) ;{ }^{4}$

(2) Para todo $1 \leq j \leq n, \bigcap_{i \neq j}$ ker $\sigma_{i} \backslash$ ker $\sigma_{j} \neq \emptyset$.

(3) $\operatorname{dim}_{\mathbb{F}_{2}}(G / \Delta)=n$.

c) Se $\Gamma$ tem codimensão $m$ em $G$ - i.e., $\operatorname{dim}_{\mathbb{F}_{2}}(G / \Gamma)=m-$, então existe $\left\{\tau_{1}, \ldots, \tau_{m}\right\} \subseteq X_{G}$, linearmente independentes, tais que $\Gamma=\bigcap_{k=1}^{m} \operatorname{ker} \tau_{k}$.

\section{Prova.}

a) Preliminarmente, faremos as considerações que seguem. Como $\Delta$ é um subgrupo saturado de índice $\leq 2^{n}$, o quociente $G / \Delta=K$ é um grupo especial finito e reduzido. Para $1 \leq i \leq n$, o Teorema 3.29.(b) fornece um SG-carácter, $\widetilde{\sigma}_{i}: K \rightarrow \mathbb{Z}_{2}$, tal que $\tilde{\sigma}_{i} \circ \pi_{\Delta}=\sigma_{i}$, onde $\pi_{\Delta}: G \rightarrow$ $K$ é o SG-morfismo quociente. Está claro que $\pi_{\Delta}^{-1}\left[\bigcap_{i=1}^{n} \operatorname{ker} \widetilde{\sigma}_{i}\right]=\Delta \operatorname{logo}$, como $\pi_{\Delta}$ é sobrejetora, $\bigcap_{i=1}^{n} \operatorname{ker} \widetilde{\sigma}_{i}=\{1\}$. Pelo Lema 3.17 em $[D M 1],\left\{\widetilde{\sigma}_{i}: 1 \leq i \leq n\right\}$ gera $\chi(K)$.

\footnotetext{
${ }^{4}$ Como usual, utilizaremos as abreviaçōes li. e l.d. para independência e dependência linear.
} 
Para provar (a) é suficiente verificar que $\Delta \subseteq$ ker $\tau$ implica que $\tau$ é uma combinação linear dos $\sigma_{i}$. Seja $\widetilde{\tau}$ o SG-carácter induzido por $\tau$ em $\bar{K}$ (como acima). Pelas considerações precedentes podemos escrever

$$
\widetilde{\tau}={\widetilde{\sigma_{1}}}^{\alpha_{1}} \ldots{\widetilde{\sigma_{n}}}^{\alpha_{n}}
$$

onde $\alpha_{i} \in\{0,1\}$ e $\widetilde{\sigma}_{i}^{\alpha_{i}}=\left\{\begin{array}{ll}1 & \text { se } \alpha_{i}=0 \\ \widetilde{\sigma_{i}} & \text { se } \alpha_{i}=1 .\end{array}\right.$ Assim, $\tau=\sigma_{1}^{\alpha_{1}} \ldots \sigma_{n}^{\alpha_{n}}$, pois para todo $a \in G$

$$
\tau(a)=\tilde{\tau}(a / \Delta)={\widetilde{\sigma_{1}}}^{\alpha_{1}}(a / \Delta) \ldots{\widetilde{\sigma_{n}}}^{\alpha_{n}}(a / \Delta)=\sigma_{1}^{\alpha_{1}}(a) \ldots \sigma_{n}^{\alpha_{n}}(a)=\left(\sigma_{1}^{\alpha_{1}} \ldots \sigma_{n}^{\alpha_{n}}\right)(a)
$$

e portanto $\tau \in\left[\sigma_{1}, \ldots, \sigma_{n}\right]$, como desejado.

b) $(1) \Rightarrow(2)$ é consequência de (a).

$(2) \Rightarrow(3):$ Para cada $1 \leq j \leq n$, escolhemos $a_{j} \in \bigcap_{i \neq j}$ ker $\sigma_{i} \backslash \operatorname{ker} \sigma_{j} ;$ então $\left\{a_{1} / \Delta, \ldots a_{n} / \Delta\right\}$ é uma base para $G / \Delta$. Para verificar este fato, primeiro note que se $\emptyset \neq S \subseteq_{f}\{1, \ldots, n\}$ e

$$
b=\Pi_{j \in S} a_{j}
$$

então para todo $j \in S$ temos $\sigma_{j}(b)=-1^{5}$, assim $b \notin \Delta$. Acabamos de verificar que nenhum produto não vazio dos $a_{j}$ está em $\Delta$. Segue imediatamente que $\left\{a_{1} / \Delta, \ldots, a_{n} / \Delta\right\}$ é 1.i. em $G / \Delta$. Para $a \in G$, definimos

$$
d(a)=a_{1}^{\frac{1-\sigma_{1}(a)}{2}} \ldots a_{n}^{\frac{1-\sigma_{n}(a)}{2}}
$$

onde $a^{\varepsilon}= \begin{cases}a & \varepsilon=1 \\ 1 & \varepsilon=0\end{cases}$

Cálculos elementares mostram que para $1 \leq j \leq n$,

$$
\sigma_{j}(d(a))=\left(\sigma_{j}\left(a_{j}\right)\right)^{\frac{1-\sigma_{j}(a)}{2}}=(-1)^{\frac{1-\sigma_{j}(a)}{2}}=\sigma_{j}(a),
$$

de onde concluímos que $a \cdot d(a) \in \Delta$. Logo, $a / \Delta=d(a) / \Delta$, e portanto $\left\{a_{1} / \Delta, \ldots, a_{n} / \Delta\right\}$ gera $G / \Delta$, estabelecendo a igualdade $\operatorname{dim}_{\mathbb{F}_{2}}(G / \Delta)=n$.

$(3) \Rightarrow(1)$ : Seja $S$ um subconjunto 1.i. maximal de $\left\{\sigma_{1}, \ldots, \sigma_{n}\right\}$; está claro que $\Delta=\bigcap_{\sigma \in S} \operatorname{ker} \sigma$. Seja $k=$ card $S$; definimos uma função

$$
h: G / \Delta \longrightarrow \mathbb{Z}_{2}{ }^{k}, \text { dada por } h(a / \Delta)=\langle\sigma(a)\rangle_{\sigma \in S}
$$

É fácil verificar que $h$ é um SG-morfismo; além disso, se $a b \notin \Delta$, existe $\sigma \in S$ tal que $\sigma(a) \neq \sigma(b)$. Logo, $h$ é injetor. Em particular, $h$ é uma aplicação linear injetora de espaços vetoriais sobre $\mathbb{F}_{2}$, o que implica que $\operatorname{dim} G / \Delta \leq k=\operatorname{dim} \mathbb{F}_{2}{ }^{k}$. Assim, por $(3), k=n$ e $\left\{\sigma_{1}, \ldots, \sigma_{n}\right\}$ é l.i. ${ }^{6}$.

c) Seja $M=G / \Gamma$ e $\pi_{\Gamma}: G \longrightarrow M$ o SG-morfismo canônico. Como $M$ é um grupo especial reduzido finito, seu espaço de ordens $X_{M}$ também é finito. O Teorema da Separação (3.30.(c)) garante que $\bigcap_{\mu \in X_{M}} \operatorname{ker} \mu=\{1\}$. Seja $S$ um subconjunto l.i. maximal de $X_{M}$; ainda temos $\bigcap_{\mu \in S}$ ker $\mu=\{1\}$. Consideremos

$$
T=\left\{\mu \circ \pi_{\Gamma}: \mu \in S\right\}
$$

\footnotetext{
${ }^{5}$ Fixado $j \in S$, temos $\sigma_{j}\left(a_{j}\right)=-1$ e $\sigma_{j}\left(a_{i}\right)=1$, para todo $i \neq j$.

${ }^{6}$ Recordemos que um subconjunto de um espaço vetorial de dimensão finita é uma base sse for um conjunto gerador mimimal sse for um conjunto l.i. maximal.
} 
Está claro que $\bigcap_{\sigma \in T} \operatorname{ker} \sigma=\Gamma$ e que $T$ é l.i. (senão, $S$ seria l.d.). Do item (b) segue que card $T=m=\operatorname{dim} M$, encerrando a prova.

Observação 6.8. O item (a) em 6.7 é falso para subconjuntos infinitos de certos $X_{G}$. Como um exemplo, seja $A=\mathbb{Z}_{2}{ }^{\omega}$ o produto enumerável do grupo especial $\mathbb{Z}_{2}$. A é um grupo especial reduzido, onde 1 e -1 são as sequências constante de valor 1 e -1 , respectivamente. Seja $\pi_{n}$ a projeção na $n^{e ́ s i m a}$ coordenada. Está claro que

$$
\Pi=\left\{\pi_{n}: n \geq 1\right\} \subseteq X_{A} .
$$

É fácil verificar (usando 6.7.(a)) que $\Pi$ é linearmente independente. Além disso, note que $\bigcap_{n \geq 1} \operatorname{ker} \pi_{n}=\{\widehat{1}\}$.

Um ultrafiltro não-principal $\mathcal{F}$ em $\omega$ induz $\sigma_{\mathcal{F}} \in X_{A}$, dada para $s \in A$ e $\alpha \in\{ \pm 1\}$, por

$$
\sigma_{\mathcal{F}}(s)=\alpha \quad \Leftrightarrow \quad\left\{k \in \omega: s_{k}=\alpha\right\} \in \mathcal{F} .
$$

Para verificar que $\sigma_{\mathcal{F}} \notin[\Pi]$ é suficiente observar que se $S \subseteq_{f} \omega$ e $s \in A$ verificam

$$
\forall k \in \omega, s_{k}=1 \quad \Leftrightarrow \quad k \in S,
$$

então $s \in \bigcap_{k \in S} \operatorname{ker} \pi_{k}$, muito embora $s \notin \operatorname{ker} \sigma_{\mathcal{F}}$, pois $\mathcal{F}$ sendo não-principal, não pode conter o conjunto finito $S$.

Sejam $G$ um grupo especial reduzido e $\varepsilon_{G}: G \longrightarrow B_{G}$ a sua envoltória Booleana (3.4.3). Como discutido no Capítulo 4, o SG-morfismo $\varepsilon_{G}$ induz uma par adjunto, constituído de aplicações crescentes

$$
\varepsilon_{G *}: \operatorname{Ssat}(G) \longrightarrow \operatorname{Ssat}\left(B_{G}\right) \quad \text { e } \quad \varepsilon_{G}^{*}: \operatorname{Ssat}\left(B_{G}\right) \longrightarrow \operatorname{Ssat}(G),
$$

onde $\varepsilon_{G *}(\Delta)=\left(\varepsilon_{G}[\Delta]\right)^{\text {sat }}$ e $\varepsilon_{G}^{*}(\Sigma)=\varepsilon_{G}^{-1}[\Sigma]$.

Lema 6.9. Sejam $G$ um grupo especial reduzido e $\varepsilon_{G}: G \longrightarrow B_{G}$ a sua envoltória Booleana. Se $\Delta \in \operatorname{Ssat}(G)$, então

$$
\varepsilon_{G *}(\Delta)=\left\{b \in B_{G}: \exists\left\{x_{1}, \ldots, x_{n}\right\} \subseteq \Delta \text { tal que } b \leq \bigcup_{j=1}^{n} \varepsilon_{G}\left(x_{i}\right)\right\},
$$

isto é, $\varepsilon_{G *}(\Delta)$ é o ideal de $B_{G} \underline{\text { gerado }}$ por $\varepsilon_{G}(\Delta)$.

Prova. Uma vez que os subgrupos saturados de $B$ são seus ideais (3.47.(c)) e que o lado direito da igualdade no enunciado é exatamente o ideal gerado por $\varepsilon_{G}(\Delta)$, concluímos que este deve ser a saturação, em $B_{G}$, de $\varepsilon_{G}(\Delta)$, estabelecendo a igualdade desejada.

Proposição 6.10. Sejam $G$ um grupo especial reduzido e $\varepsilon_{G}: G \longrightarrow B_{G}$ a sua envoltória Booleana. 
a) Para $\sigma_{1}, \ldots, \sigma_{n} \subseteq X_{G}$, seja $\left\{\sigma_{j_{*}}: 1 \leq j \leq n\right\} \subseteq X_{B_{G}}$ as extensões dadas por 3.48.(c). Se $\Delta$ $=\bigcap_{i=1}^{n} \operatorname{ker} \sigma_{i}$, entâo

$$
\varepsilon_{G *}(\Delta)=\bigcap_{i=1}^{n} \operatorname{ker} \sigma_{i_{*}} .
$$

b) Se $\Sigma$ é um ideal de indice finito em $B_{G}$, então

$$
\varepsilon_{G *}\left(\varepsilon_{G}^{*}(\Sigma)\right)=\Sigma .
$$

c) Opardual $\left\langle\varepsilon_{G_{*}}, \varepsilon_{G}^{*}\right\rangle$ estabelece correspondências crescentes, biunívocas e inversas entre $\mathcal{F}(G)$ e $\mathcal{F}\left(B_{G}\right)^{7}$.

\section{Prova.}

a) Como cada $\sigma_{i_{*}}$ é um Ba-morfismo (6.3), seus núcleos são ideais; além disso, uma vez que $\sigma_{i_{*}}$ é a extensão de $\sigma_{i}$ ao longo de $\varepsilon_{G}$, está claro que $\varepsilon_{G_{*}}(\Delta) \subseteq \bigcap_{i=1}^{n}$ ker $\sigma_{i *}$. Para provar a inclusão recíproca, suponha, por absurdo, que existe $y \in B_{G}$ such that

$$
y \in\left(\bigcap_{i=1}^{n} \operatorname{ker} \sigma_{i_{*}}\right) \backslash \varepsilon_{G *}(\Delta) .
$$

Como $\varepsilon_{G *}(\Delta)$ é um ideal em $B_{G}$, é um subgrupo saturado. Logo, o Teorema da separação (3.30.(c)) fornece um SG-carácter, $\alpha: B_{G} \longrightarrow \mathbb{Z}_{2}$, tal que $\varepsilon_{G *}(\Delta) \subseteq \operatorname{ker} \alpha$ e $\alpha(y)=-1$. Se $\tau=\alpha \circ \varepsilon_{G}$, então $\tau \in X_{G}$ e a unicidade da extensão em 3.48.(c) garante que $\alpha=\tau_{*}$. Note que temos

$$
\Delta=\bigcap_{i=1}^{n} \operatorname{ker} \sigma_{i} \subseteq \operatorname{ker} \tau
$$

e portanto 6.7.(a) implica que $\tau$ é uma combinação linear de $\left\{\sigma_{1}, \ldots, \sigma_{n}\right\}$. Mas então, a unicidade da extensão acarreta que $\alpha=\tau_{*}$ é uma combinação linear de $\left\{\sigma_{1 *}, \ldots, \sigma_{n *}\right\}$, o que é impossível pois $\sigma_{i *}(y)=1,1 \leq i \leq n$, enquanto que $\alpha(y)=-1$.

b) Como $\Sigma$ tem índice finito em $B_{G}$, também tem codimensão finita em $B_{G}{ }^{8}$. Por 6.7.(c), existe $\left\{\tau_{1}, \ldots, \tau_{m}\right\} \subseteq X_{B_{G}}$ tal que

$$
\Sigma=\bigcap_{j=1}^{m} \operatorname{ker} \tau_{j}
$$

Para cada $1 \leq j \leq m$, consideremos $\sigma_{j}=\tau_{j} \circ \varepsilon_{G}$; então $\sigma_{j} \in X_{G}$ e unicidade da extensão em 3.48.(c) garante que

$$
\text { Para } 1 \leq j \leq m, \quad \tau_{j}=\sigma_{j_{*}} .
$$

Está claro que $\varepsilon_{G}^{*}(\Sigma)=\bigcap_{j=1}^{m} \operatorname{ker} \sigma_{j}$. Mas então o item (a) implica

$$
\varepsilon_{G *}\left(\varepsilon_{G}^{*}(\Sigma)\right)=\bigcap_{j=1}^{n} \operatorname{ker} \sigma_{j_{*}}=\bigcap_{j=1}^{m} \operatorname{ker} \tau_{j}=\Sigma,
$$

como desejado.

c) Uma vez que $\varepsilon_{G}$ é uma imersão completa (3.48.(a)), a Proposição 4.2.(c) garante que para todo $\Delta \in \operatorname{Ssat}(G)$ temos

$$
\varepsilon_{G}^{*}\left(\varepsilon_{G *}(\Delta)\right)=\Delta
$$

A conclusão agora segue imediatamente de (b), encerrando a prova.

\footnotetext{
${ }^{7} \mathcal{F}(G)$ é o conjunto dos subgrupos saturados de índice (ou codimensão) finito em $G$ (4.8.(c)).

${ }^{8}$ Como $B_{G} / \Sigma$ é um espaço vetorial sobre $\mathbb{F}_{2}$, card $\left(B_{G} / \Sigma\right)=2^{k}$, onde $k$ é a codimensão de $\Sigma$.
} 
Observação 6.11. Na Proposição 4.15.(b) em [DM1] é provado, com outra notação, que o item (c) em 6.10 vale para subgrupos saturados maximais em $G$ e ideais maximais em $B_{G}$. Assim, 6.10.(b) generaliza este resultado para objetos saturados de índice finito. Por outro lado, pela Proposição $7.28 \mathrm{em}[\mathbf{D M 1}], \varepsilon_{G_{*}}$ e $\varepsilon_{G}^{*}$ são correspondências biunívocas e inversas entre $\operatorname{Ssat}(G)$ e $\operatorname{Ssat}\left(B_{G}\right)$ sse $G$ é uma álgebra de Boole.

\subsection{A Envoltória Profinita de uma Álgebra de Boole}

Começamos com a seguinte

Proposição 6.12. Se $G$ é um grupo especial reduzido, as seguintes condições são equivalentes :

(1) $\mathcal{P}(G)$ é uma álgebra de Boole;

(2) G é uma álgebra de Boole;

(3) $\forall \Delta \in S s a t(G), \quad G / \Delta$ é uma álgebra de Boole;

(4) $\forall \Delta \in \mathcal{F}(G), G / \Delta$ é uma álgebra de Boole.

\section{Prova.}

$\underline{(1) \Rightarrow(2)}$ : A Proposição 7.17 em [DM1] garante que úm grupo especial é uma álgebra de Boole sse para cada $a, b \in G$, a forma $\langle 1, a, b,-a b\rangle$ é isotrópica:

Para $a, b \in G$, seja $\varphi=\langle 1, a, b,-a b\rangle$ e considere a forma

$$
\eta_{G} \star \varphi=\left\langle\eta_{G}(1), \eta_{G}(a), \eta_{G}(b),-\eta_{G}(a) \eta_{G}(b)\right\rangle .
$$

Como $\mathcal{P}(G)$ é uma álgebra de Boole e $\eta_{G}$ reflete subformas (5.43.(a)), $\eta_{G}$ reflete isotropia e portanto $\varphi$ é isotrópica em $G$. Pelo critério mencionado acima, $G$ é uma álgebra de Boole.

$(2) \Rightarrow(3)$ : Como a família dos subgrupos saturados de $G$ coincide com a família dos ideais em $\overline{G(3.47 .(c)})$ e o quociente de uma álgebra de Boole por um ideal é uma álgebra de Boole, (3) segue imediatamente.

Está claro que $(3) \Rightarrow(4)$, enquanto que $(4) \Rightarrow(1)$ é consequência do Lema 5.37 e do fato que

$$
\mathcal{P}(G)=\lim _{\Delta \in \mathcal{F}(G)} G / \Delta
$$

encerrando a prova.

6.13. A álgebra de Boole profinita $\mathbf{2}^{\boldsymbol{X}}$. Retomamos, em mais generalidade, o tema de 6.2.

Seja $X$ um conjunto. Se dermos a $T=2^{X}$ a topologia e a estrutura produto, obtemos uma álgebra de Boole topológica, cujo espaço subjacente é Booleano. Está claro que $T$ é limite projetivo de grupos finitos (os produtos sobre subconjuntos finitos de $X$ ), e portanto um grupo 
especial reduzido e profinito $(5.8,5.11)$. No entanto, é fácil verificar este fato diretamente, utilizando o critério do Teorema 5.17 :

* $\equiv_{T}$ é fechada em $T^{4}$ com a topologia produto: Sejam $s_{1}, s_{2}, s_{3}, s_{4} \in T$ tais que

$$
\left\langle s_{1}, s_{2}\right\rangle \not \equiv T\left\langle s_{3}, s_{4}\right\rangle \text {. }
$$

Como a isometria em $T$ é definida coordenada a coordenada, existe $x \in X$ tal que

$$
\left\langle s_{1}(x), s_{2}(x)\right\rangle \neq_{2}\left\langle s_{3}(x), s_{4}(x)\right\rangle .
$$

Considere os abertos de $T$ dados por

$$
V_{i}=\left\{t \in T: t(x)=s_{i}(x)\right\}, \quad 1 \leq i \leq 4 .
$$

Está claro que $V=\prod_{i=1}^{4} V_{i}$ é uma vizinhança aberta de $\left\langle s_{1}, s_{2}, s_{3}, s_{4}\right\rangle$ em $T^{4}$ e que para todo $\langle a, b, c, d\rangle \in V$, temos $\langle a, b\rangle \neq_{T}\langle c, d\rangle$, mostrando que o complemento de $\equiv_{T}$ é aberto em $T^{4}$.

* Existe um pf-sistema em T: Para cada $S \subseteq_{f} X$, considere

$$
I_{S}=\{t \in T: \forall x \in S, \quad t(x)=0\} .
$$

Está claro que $I_{S}$ é um subgrupo aberto e fechado de $T$, de índice finito ${ }^{9}$. Para vermos que é saturado, basta notar que trata-se de um ideal, e portanto, por 3.47.(c), $I_{S}$ é saturado em $T$. Finalmente, como a unidade do grupo $T$ é a sequência constante de valor 0 , está claro que

$$
\mathcal{N}=\left\{I_{S}: S \subseteq_{f} X\right\}
$$

é um sistema fundamental de vizinhanças de 1 em $T$ e assim o pf-sistema desejado.

\subsection{Construção.}

a) Sejam $A$ uma álgebra de Boole e $X_{A}$ seu espaço de ordens (ou de Stone; 6.4). Para $a \in A$ definimos

$$
\check{a}: X_{A} \longrightarrow 2 \text {, dada por } \check{a}(\mathcal{U})= \begin{cases}0 & \text { se } a \notin \mathcal{U} ; \\ 1 & \text { se } a \in \mathcal{U} .\end{cases}
$$

Note que, com a notação em 6.4, ă é simplesmente a função característica de $X_{a}$. Seja

$$
\mathfrak{c}_{A}: A \longrightarrow 2^{X_{A}} \text {, dada por } \mathfrak{c}_{A}(a)=\check{a} .
$$

b) Se $f: A \longrightarrow B$ é um Ba-morfismo, sabemos da dualidade de Stone (6.5.(b)) que $f$ induz uma função contínua

$$
X(f): X_{B} \longrightarrow X_{A} .
$$

Por composição, obtemos uma função

\footnotetext{
${ }^{9} T / I_{S} \cong 2^{S}$.
} 
$\rho_{f}: 2^{X_{A}} \longrightarrow 2^{X_{B}}$, dada por $\rho_{f}(t)=t \circ X(f)$

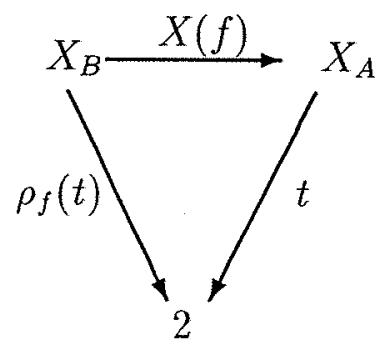

A notação estabelecida aqui será utilizada constantemente em tudo que segue.

Proposição 6.15. Sejam A uma álgebra de Boole e $f: A \longrightarrow B$ um Ba-morfismo. Com a notação em 6.13 e 6.14 temos:

a) $\mathfrak{c}_{A}: A \longrightarrow 2^{X_{A}}$ é um Ba-morfismo injetor.

b) Se $S \subseteq_{f} X_{A}$, então $\mathfrak{c}_{A}^{-1}\left(I_{S}\right)=\bigcap_{\mathcal{U} \in S}-\mathcal{U}$.

c) A função $\rho_{f}: 2^{X_{A}} \longrightarrow 2^{X_{B}}$ é um Ba-morfismo tal que para todo $R \subseteq_{f} X_{B}$

$$
\rho_{f}^{-1}\left(I_{R}\right)=I_{X(f)(R)} \text {. }
$$

Em particular, $\rho_{f}$ é contínuo. Além disso, o seguinte diagrama é comutativo:

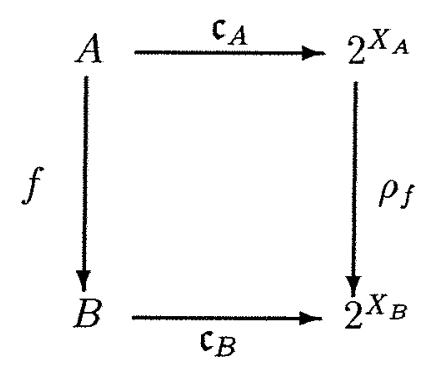

d) Se fé injetor, então $\rho_{f}$ é uma imersão pura e contínua.

\section{Prova.}

a) Sejam $a, c \in A$; se $\mathfrak{c}_{A}(a)=\mathfrak{c}_{A}(c)$ então para todo ultrafiltro $\mathcal{U} \in X_{A}$, temos

$$
a \in \mathcal{U} \Leftrightarrow c \in \mathcal{U}
$$

Como ultrafiltros separam pontos em $A$, obtemos $a=c$ e $\mathfrak{c}_{A}$ é injetora. Está claro que

$$
\left\{\begin{array}{l}
\mathfrak{c}_{A}(\perp)=\check{I}=\text { a função constante } 0 \\
\mathfrak{c}_{A}(T)=\check{T}=\text { a função constante } 1
\end{array}\right.
$$

mostrando que $\mathfrak{c}_{A}$ leva $\perp \mathrm{em} \perp \mathrm{e} T$ em $T$. Para $a, c \in A$, cálculos elementares verificarão que

$$
(a \vee c) \check{c}=\check{a} \vee \check{c} \quad \text { e }(a \wedge c) \check{c}=\check{a} \wedge \check{c}
$$

e portanto $\mathfrak{c}_{A}$ é um Ba-morfismo. Finalmente suponha que $a \in D_{A}(1, b)$; isto significa que $a \leq$ $b$ em $A$ e portanto todo ultrafiltro que contém $a$ deve conter $b$. Logo, $X_{a} \subseteq X_{b}$ e assim $\check{a} \leq \check{b}$, mostrando que $\check{a} \in D_{2} x_{A}(1, \check{b})$ e encerrando a prova de (a). 
b) Como $I_{S}=\left\{t \in 2^{X_{A}}: t(\mathcal{U})=0, \forall \mathcal{U} \in S\right\}$, para $a \in A$, obtemos, lembrando 6.1.(b):

$$
\check{a} \in I_{S} \quad \Leftrightarrow \quad a \notin \mathcal{U} \text {, para todo } \mathcal{U} \in S \quad \Leftrightarrow \quad a \in \bigcap_{\mathcal{U} \in S}-\mathcal{U},
$$

como necessário.

c) É elementar verificar que $\rho_{f}$ é um Ba-morfismo. Para $R \subseteq_{f} X_{B}$ e $t \in 2^{X_{A}}$, temos

$$
\begin{aligned}
\rho_{f}(t) \in I_{R} & \Leftrightarrow t \circ X(f) \in I_{R} \quad \Leftrightarrow \quad t\left(X(f)\left(\mathcal{U}^{\prime}\right)\right)=0, \quad \forall \mathcal{U}^{\prime} \in R \\
& \Leftrightarrow t(\mathcal{U})=0, \quad \forall \mathcal{U} \in X(f)(R) \Leftrightarrow \quad \Leftrightarrow \quad t \in I_{X(f)(R)},
\end{aligned}
$$

como desejado. A relação $\rho_{f}^{-1}\left(I_{R}\right)=I_{X(f)(R)}$ implica, tendo em vista que as famílias (6.13)

$$
\left\{I_{R}: R \subseteq_{f} X_{B}\right\} \quad \text { e } \quad\left\{I_{S}: S \subseteq_{f} X_{A}\right\}
$$

são pf-sistemas em $2^{X_{B}}$ e $2^{X_{A}}$, respectivamente, que $\rho_{f}$ é contínua na função identicamente nula e portanto contínua, pois é um morfismo de grupos (5.2.(c)). A verificação que o diagrama do enunciado é comutativo é outra versão de 6.5.(b). Sejam $a \in A$ e $\mathcal{U}^{\prime} \in X_{B}$; então

$$
\begin{aligned}
\check{a}\left(X(f)\left(\mathcal{U}^{\prime}\right)\right)=1 & \Leftrightarrow a \in X(f)\left(\mathcal{U}^{\prime}\right) \Leftrightarrow a \in f^{-1}\left(\mathcal{U}^{\prime}\right) \\
& \Leftrightarrow f(a) \in \mathcal{U}^{\prime} \Leftrightarrow[f(a)]^{\prime}\left(\mathcal{U}^{\prime}\right)=1 .
\end{aligned}
$$

Uma vez que

$$
\rho_{f} \circ \mathfrak{c}_{A}(a)=\rho_{f}(\check{a})=\check{a} \circ X(f) \text { e } \quad \mathfrak{c}_{B} \circ f(a)=[f(a) \check{]}
$$

a equivalência em $\left(^{*}\right)$ garante que $\rho_{f} \circ \mathfrak{c}_{A}=\mathfrak{c}_{B} \circ f$, como desejado.

d) Se $f$ é injetora, sabemos de 6.5.(c) que $X(f)$ é sobrejetora. Neste caso, é imediato que $\rho_{f}$ é injetora. Pela Proposição 6.6, $\rho_{f}$ é uma imersão pura, encerrando a prova.

Por 5.37.(b), se $A$ é uma álgebra de Boole, $\mathcal{P}(A)$ é uma álgebra de Boole completa. Podemos, no entanto ser mais específicos, conforme mostra o principal resultado desta seção :

\section{Teorema 6.16.}

a) Se A é uma álgebra de Boole, seja $X_{A}$ seu espaço de ordens e $2^{X_{A}}$ álgebra de Boole profinita associada como em 6.13. Então existe um único Ba-isomorfismo contínuo

$$
\beta_{A}: \mathcal{P}(A) \longrightarrow 2^{X_{A}}
$$

tal que o triângulo abaixo é comutativo
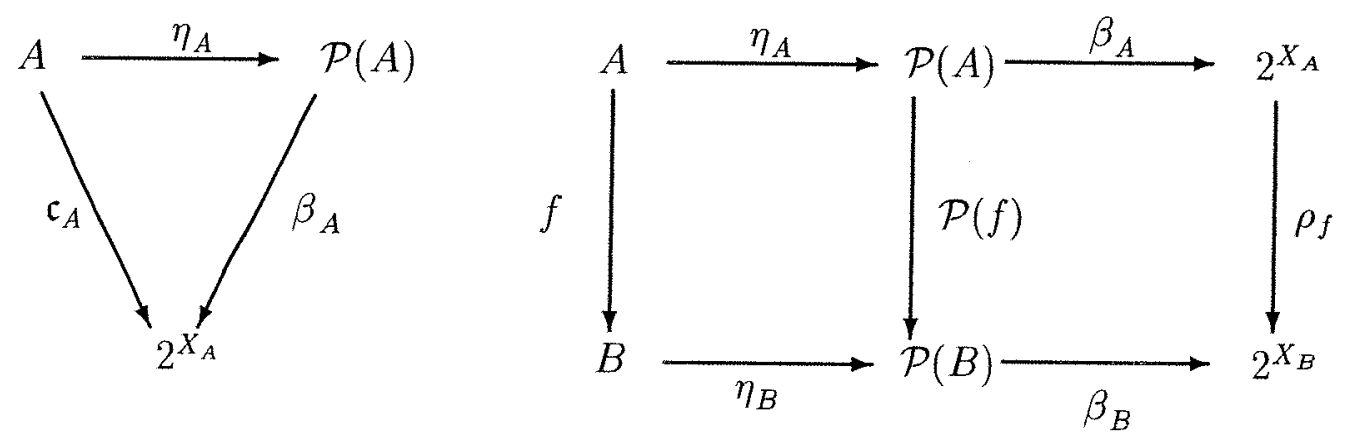

b) Se $f: A \longrightarrow B$ é um Ba-morfismo, então o diagrama acima a direita é comutativo. Em 
particular, se $f$ é injetor então $\mathcal{P}(f)$ é uma imersão pura ${ }^{10}$ e contínua.

\section{Prova.}

a) Utilizaremos, mais uma vez, o critério em 5.43.(c). Consideremos o Ba-morfismo injetor $(6.15 .(\mathrm{a}))$

$$
\mathfrak{c}_{A}: A \longrightarrow 2^{X_{A}}
$$

Vamos verificar que $\boldsymbol{c}_{A}$ satisfaz as condições (1) e (2) em 5.43.(c).

(1) $\eta_{A}$ é contínua na topologia induzida por $2^{X_{A}}$ em $A$, via $\mathfrak{c}_{A}:$ Por $\left(1^{*}\right)$ na Observação 5.46.(c), e levando em conta (6.13) que

$$
\mathcal{N}=\left\{I_{S}: S \subseteq_{f} X_{A}\right\}
$$

é um pf-sitema em $2^{X_{A}}$, é suficiente mostrar que

$$
\text { Para todo } J \in \mathcal{F}(A) \text {, existe } S \subseteq f X_{A} \text { tal que } \mathfrak{c}_{A}^{-1}\left(I_{S}\right) \subseteq J
$$

Como $J$ é um ideal de índice finito em $A$, por 6.7.(c) existe $\left\{\tau_{1}, \ldots, \tau_{m}\right\} \subseteq X_{A}$ tal que

$$
J=\bigcap_{i=1}^{m} \operatorname{ker} \tau_{i}
$$

Como os $\operatorname{ker} \tau_{i}$ são ideais maximais em $A$, segue de 6.1.(b) que

$$
S=\left\{-\operatorname{ker} \tau_{i}: 1 \leq i \leq m\right\}
$$

é um subconjunto finito de $X_{A}$. De 6.15.(b) e (II) obtemos

$$
\mathfrak{c}_{A}^{-1}\left(I_{S}\right)=\bigcap_{\mathcal{U} \in S}-\mathcal{U}=\bigcap_{i=1}^{m} \operatorname{ker} \tau_{i}=J
$$

estabelecendo (I) e completando a verificação da condição (1).

(2) A imagem de $\equiv_{A}$ por $\mathfrak{c}_{A}^{4}$ é densa em $\equiv_{2} x_{A}:$ Seja $S=\left\{\mathcal{U}_{1}, \ldots, \mathcal{U}_{n}\right\}$ um subconjunto finito de $X_{A}$ e $t_{1}, t_{2}, t_{3}, t_{4} \in 2^{X_{A}}$ tais que

$$
\left\langle t_{1}, t_{2}\right\rangle \equiv_{2} x_{A} \quad\left\langle t_{3}, t_{4}\right\rangle
$$

Como $X_{A}$ é um espaço Hausdorff, 6.5.(a).(2) garante que existe $\left\{w_{1}, \ldots, w_{n}\right\} \subseteq A$ tal que

$$
\text { - Para } i \neq j, w_{i} \wedge w_{j}=\perp ; \quad-\operatorname{Para} 1 \leq i \leq n, \quad w_{i} \in \mathcal{U}_{i} \text {. }
$$

Como todos os $\mathcal{U}_{i}$ são filtros próprios, temos que $w_{i} \neq 1,1 \leq i \leq n$. Para $1 \leq p \leq 4$, seja

$$
\alpha(p)=\left\{k \in\{1, \ldots, n\}: t_{p}\left(\mathcal{U}_{k}\right)=1\right\}
$$

e definimos

$$
a_{p}=\bigvee_{i \in \alpha(p)} w_{i}
$$

Note que $a_{p}$ é um supremo de elementos dois a dois disjuntos em $A$.

Fato 6.17. Com a notação acima

a) Para todo $1 \leq k \leq n$ e todo $1 \leq p \leq 4$

\footnotetext{
${ }^{10}$ Note que um morfismo de grupos especiais reduzidos provenientes de álgebras booleanas é $L_{S G}$-imersão pura sse for uma $L_{B A}$-imersão pura sse for injetor.
} 
$\check{a}_{p}\left(\mathcal{U}_{k}\right)=1 \stackrel{(i)}{\Leftrightarrow} w_{k} \leq a_{p} \stackrel{(i i)}{\Leftrightarrow} t_{p}\left(\mathcal{U}_{k}\right)=1$.

b) Para $1 \leq k \leq n, \quad\left\{\begin{array}{l}w_{k} \leq a_{1} \vee a_{2} \Leftrightarrow w_{k} \leq a_{1} \text { ou } w_{k} \leq a_{2} \\ w_{k} \leq a_{3} \vee a_{4} \Leftrightarrow w_{k} \leq a_{3} \text { ou } w_{k} \leq a_{4} .\end{array}\right.$

Prova.

a) Prova da equivalência (i) : Fixemos $p$ entre 1 e 4 e $k \in\{1, \ldots, n\}$; pela definição de $\check{a}_{p}$ temos que

$$
\check{a}_{p}\left(\mathcal{U}_{k}\right)=1 \Leftrightarrow a_{p} \in \mathcal{U}_{k} \quad \Leftrightarrow \quad \bigvee_{i \in \alpha(p)} w_{i} \in \mathcal{U}_{k}
$$

Como os $w_{i}$ são dois a dois disjuntos, existe um único $i \in \alpha(p)$ tal que $w_{i} \in \mathcal{U}_{k}$. Pela construção dos $w_{i}$, devemos ter $i=k$, isto é, $k \in \alpha(p)$ e portanto $w_{k} \leq a_{p}$. Está claro que $w_{k} \leq a_{p}$ implica $a_{p} \in \mathcal{U}_{k}$, e $\operatorname{assim} \check{a}_{p}\left(\mathcal{U}_{k}\right)=1$.

A equivalência em (ii) é consequência direta das definições de $\alpha(p)$ e $a_{p}$ em (III) e (IV).

b) É suficiente verificar a implicação $(\Rightarrow)$. Temos

$$
a_{1} \vee a_{2}=\bigvee_{i \in \alpha(1)} w_{i} \vee \bigvee_{j \in \alpha(2)} w_{j}
$$

Assim, se $w_{k} \leq a_{1} \vee a_{2}$, a lei distributiva fornece

$$
w_{k}=w_{k} \wedge\left(a_{1} \vee a_{2}\right)=\bigvee_{i \in \alpha(1)} w_{k} \wedge w_{i} \vee \bigvee_{j \in \alpha(2)} w_{k} \wedge w_{j}
$$

Como os $w_{i}$ são dois a dois disjuntos, se $k \notin \alpha(1) \cup \alpha(2)$, o supremo à direita em (V) é igual a $\perp$, o que é impossivel pois $w_{k} \neq \perp$. Consequentemente, $w_{k} \leq a_{1}$ ou $w_{k} \leq a_{2}$. Analogamente, tratamos o caso de $a_{3} \vee a_{4}$ e a prova do Fato está terminada.

Segue do item (a) em 6.17 que para todo $1 \leq k \leq n$ e $1 \leq p \leq 4$ temos

$$
\check{a}_{p}\left(\mathcal{U}_{k}\right)=t_{p}\left(\mathcal{U}_{k}\right) \text {. }
$$

Portanto, $\left\langle\mathfrak{c}_{A}\left(a_{1}\right), \mathfrak{c}_{A}\left(a_{2}\right), \mathfrak{c}_{A}\left(a_{3}\right), \mathfrak{c}_{A}\left(a_{4}\right)\right\rangle \in t_{1} I_{S} \times t_{2} I_{S} \times t_{3} I_{S} \times t_{4} I_{S}$.

Resta mostrar que $\left\langle a_{1}, a_{2}\right\rangle \equiv_{A}\left\langle a_{3}, a_{4}\right\rangle$; pela Proposição 3.47 e o parágrafo que a precede, esta relação é equivalente a

$$
a_{1} \vee a_{2}=a_{3} \vee a_{4} \quad \text { e } \quad a_{1} \wedge a_{2}=a_{3} \wedge a_{4}
$$

Uma vez que $a_{p}, 1 \leq p \leq 4$, são uniões disjuntas dos $w_{i}$, (VII) é equivalente a

$$
\forall 1 \leq k \leq n, \quad\left\{\begin{array}{l}
w_{k} \leq a_{1} \vee a_{2} \Leftrightarrow w_{k} \leq a_{3} \vee a_{4} \\
w_{k} \leq a_{1} \wedge a_{2} \Leftrightarrow w_{k} \leq a_{3} \vee a_{4} .
\end{array}\right.
$$

Para verificar a primeira condição em (VIII), suponha que $w_{k} \leq a_{1} \vee a_{2}$. Pelo Fato 6.17.(b), isto significa que $w_{k} \leq a_{1}$ ou $w_{k} \leq a_{2}$. De (VI) e 6.17.(a) obtemos

$$
t_{1}\left(\mathcal{U}_{k}\right) \vee t_{2}\left(\mathcal{U}_{k}\right)=\check{a}_{1}\left(\mathcal{U}_{k}\right) \vee \check{a}_{2}\left(\mathcal{U}_{k}\right)=1 .
$$

Como $\left\langle t_{1}, t_{2}\right\rangle \equiv_{2} X_{A}\left\langle t_{3}, t_{4}\right\rangle$ e a isometria em $2^{X_{A}}$ é definida ponto a ponto (6.13), concluímos que 


$$
\check{a}_{3}\left(\mathcal{U}_{k}\right) \vee \check{a}_{4}\left(\mathcal{U}_{k}\right)=t_{3}\left(\mathcal{U}_{k}\right) \vee t_{4}\left(\mathcal{U}_{k}\right)=1
$$

e portanto $\check{a}_{3}\left(\mathcal{U}_{k}\right)=1$ ou $\check{a}_{4}\left(\mathcal{U}_{k}\right)=1$. Assim, 6.17.(a) implica $w_{k} \leq a_{3} \vee a_{4}$. Uma vez que o raciocínio é claramente simétrico, concluímos que $a_{1} \vee a_{2}=a_{3} \vee a_{4}$, estabelecendo a primeira condição em (VIII). A segunda pode ser obtida pelo mesmo método e omitimos os detalhes. Isto termina a verificação da condição (2).

Por 5.43.(c), existe um único SG-isomorfismo contínuo, ou equivalentemente, um único Baisomorfismo contínuo,

$$
\beta_{A}: \mathcal{P}(A) \longrightarrow 2^{X_{A}}
$$

que faz comutar o triângulo no enunciado, completando a prova de (a).

b) Como o primeiro quadrado no diagrama a direita no enunciado é comutativo, é suficiente verificar que

$$
\rho_{f} \circ \beta_{A}=\beta_{B} \circ \mathcal{P}(f) .
$$

Como os triângulos do enunciado comutam tanto para $A$ quanto para $B$, temos

$* \beta_{B} \circ \mathcal{P}(f) \circ \eta_{A}=\beta_{B} \circ \eta_{B} \circ f=\mathfrak{c}_{B} \circ f$;

$* \rho_{f} \circ \beta_{A} \circ \eta_{A}=\rho_{f} \circ \mathfrak{c}_{A}$.

Segue imediatamente dessas igualdades e da comutatividade do quadrado em 6.15.(c) que

$$
\rho_{f} \circ \beta_{A} \circ \eta_{A}=\beta_{B} \circ \mathcal{P}(f) \circ \eta_{A} .
$$

Como as composições à esquerda de $\eta_{A}$ são contínuas e $\eta_{A}$ tem imagem densa em $\mathcal{P}(A)$, a igualdade acima implica (IX).

Se $f$ é injetor, 6.15.(d) mostra que $\rho_{f}$ é uma imersão pura. Uma vez que $\beta_{A}, \beta_{B}$ são Baisomorfismos, $\mathcal{P}(f)$ também é uma imersão pura e contínua de álgebras de Boole completas, encerrando a prova.

Observação 6.18. O Teorema 6.16 fornece uma descrição clara e funtorial da envoltória profinita de álgebras de Boole. Com a notação em 6.16, construímos uma transformação natural

$$
\beta=\left\{\beta_{A}: A \text { é uma álgebra de Boole }\right\}
$$

tal que $\beta_{A}$ é um Ba-isomorfismo bicontínuo; além disso, se $f: A \longrightarrow B$ é um Ba-morfismo, $\beta$ leva o diagrama comutativo abaixo à esquerda no diagrama comutativo a direita :
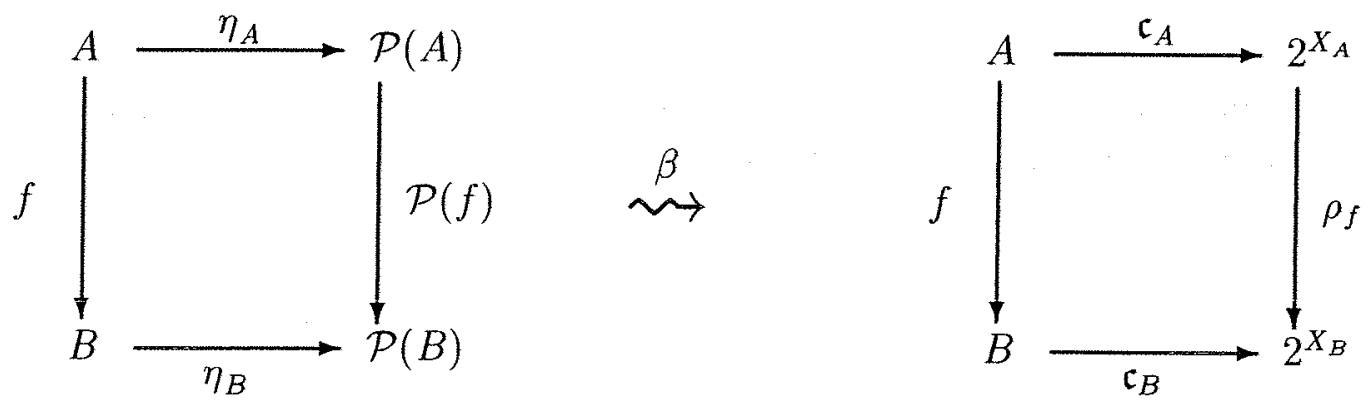
Como o diagrama acima à esquerda é o característico da envoltória profinita de uma álgebra de Boole, é lícito, quando for conveniente, subtituí-lo pelo da direita, identificando-os via o isomorfismo natural $\beta$.

Por esta identificação, o BA-morfismo canônico $\eta_{A}$ é levado em $\mathfrak{c}_{A}$ e o morfismo contínuo $\mathcal{P}(f)$ em $\rho_{f}$. Assim, se $A$ é uma álgebra de Boole, a imersão $\eta_{A}$ codifica as flechas $a, a \in A$, que determinam a topologia do espaço de Stone de $A$.

\subsection{A Preservação de Imersões Completas}

Esta seção tem a finalidade de mostrar que o funtor $\mathcal{P}$ preserva imersões completas. Se

$$
f: G \longrightarrow H
$$

é uma imersão completa e $\Delta$ é um subgrupo saturado de índice finito em $G$, em geral não podemos garantir que $f_{*}(\Delta)$ tenha índice finito em $H$. Por outro lado, pela Proposição 6.10.(a), este fato vale para a imersão completa associada à envoltória Booleana de um grupo reduzido. Assim, a tática que adotaremos para mostrar que $\mathcal{P}$ preserva imersões completas é primeiro provar que

$$
\mathcal{P}\left(\varepsilon_{G}\right): \mathcal{P}(G) \longrightarrow \mathcal{P}\left(B_{G}\right)
$$

é uma imersão completa e a partir deste resultado estabelecer o caso geral. No processo identificaremos a envoltória Booleana da envoltória profinita de um grupo reduzido.

Teorema 6.19. Sejam $G$ um grupo especial reduzido e $\varepsilon_{G}: G \longrightarrow B_{G}$ a sua envoltória Booleana.

a) $\mathcal{P}\left(\varepsilon_{G}\right): \mathcal{P}(G) \longrightarrow \mathcal{P}\left(B_{G}\right)$ é uma imersão completa e contínua.

b) A envoltória Booleana de $\mathcal{P}(G), B_{\mathcal{P}(G)}$, é a subálgebra de $\mathcal{P}\left(B_{G}\right)$ gerada por $\mathcal{P}\left(\varepsilon_{G}\right)(\mathcal{P}(G))$.

\section{Prova.}

a) $\operatorname{Sejam} \varphi, \psi$ formas de mesma dimensão sobre $\mathcal{P}(G)$, tais que

$$
\mathcal{P}\left(\varepsilon_{G}\right) \star \varphi \equiv \mathcal{P}\left(B_{G}\right) \quad \mathcal{P}\left(\varepsilon_{G}\right) \star \psi .
$$

Precisamos mostrar que $\varphi \equiv_{\mathcal{P}(G)} \psi$. Seja

$$
\iota: \mathcal{P}(G) \longrightarrow H==_{\text {def }} \prod_{\Delta \in \mathcal{F}(G)} G / \Delta
$$

a imersão canônica que identifica o limite projetivo $\mathcal{P}(G)$ com um subgrupo do produto dos quocientes $G / \Delta, \Delta \in \mathcal{F}(G)$; por 5.11.(c) sabemos que $\iota$ reflete subformas, sendo portanto uma imersão completa (3.21.(b)). Assim, para verificar que $\varphi \equiv_{\mathcal{P}(G)} \psi$, é suficiente mostrar que

$$
\iota \star \varphi \equiv_{H} \quad \iota \star \psi
$$

Como a isometria em $H$ está determinada coordenada a coordenada e $\mathfrak{p}_{\Delta}=\pi_{\Delta} \circ \iota$ (veja 5.41.(VI)), (II) é equivalente a

$$
\text { Para todo } \Delta \in \mathcal{F}(G), \mathfrak{p}_{\Delta} \star \varphi \equiv_{G / \Delta} \mathfrak{p}_{\Delta} \star \psi,
$$


que é o que passaremos a provar. Seja $\Delta$ um subgrupo saturado de índice finito em $G$. Por 6.10.(a),

$$
J=\varepsilon_{G *}(\Delta)
$$

é um ideal de índice finito em $B_{G}$. Uma vez que $\varepsilon_{G}$ é uma imersão completa, segue de 4.2.(c) que

$$
\Delta=\varepsilon_{G}^{*}\left(\varepsilon_{G *}(\Delta)\right) .
$$

Além disso, 4.6.(d) garante que o morfismo derivado

$$
f_{J}: G / \Delta \longrightarrow B_{G} / J
$$

é uma imersão completa. A construção do funtor $\mathcal{P}$ no Teorema 5.52 mostra que o SG-morfismo $\mathcal{P}\left(\varepsilon_{G}\right)$ faz comutar o seguinte diagrama (veja o diagrama $(\mathcal{P})$, na prova de 5.52 , página 162) :

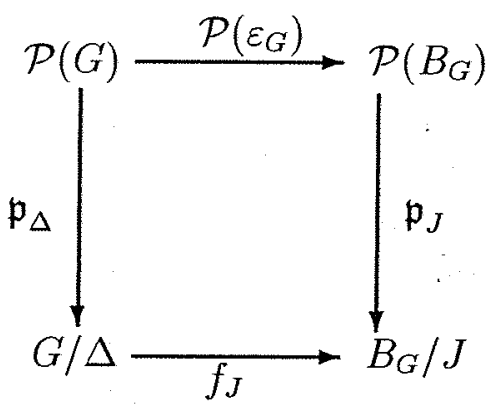

Segue de (I) e do fato que $\mathfrak{p}_{J}$ é um SG-morfismo (ou mesmo um Ba-morfismo) que

$$
\left[\mathfrak{p}_{J} \circ \mathcal{P}\left(\varepsilon_{G}\right)\right] \star \varphi \quad \equiv_{B_{G} / J}\left[\mathfrak{p}_{J} \circ \mathcal{P}\left(\varepsilon_{G}\right)\right] \star \psi .
$$

A comutatividade do diagrama acima e (IV) acarretam

$$
\left[f_{J} \circ \mathfrak{p}_{\Delta}\right] \star \varphi \equiv \equiv_{B_{G} / J}\left[f_{J} \circ \mathfrak{p}_{\Delta}\right] \star \psi .
$$

Como $f_{J}$ é uma imersão completa, a isometria precedente fornece (III), estabelecendo (a).

b) $\mathrm{O}$ método aqui é o mesmo que na Proposição 5.40.(b). Seja $\varepsilon_{\mathcal{P}(G)}: \mathcal{P}(G) \longrightarrow B_{\mathcal{P}(G)}$ a envoltória Booleana de $\mathcal{P}(G)$. Já que $\mathcal{P}\left(\varepsilon_{G}\right)$ é uma imersão completa, o item (3) do Teorema 5.2 em [DM1] garante que $B\left(\mathcal{P}\left(\varepsilon_{G}\right)\right)$ é um BA-morfismo injetor. A propriedade universal da envoltória Booleana no item (4) do Teorema 4.17 em [DM1] implica que o seguinte diagrama é comutativo :

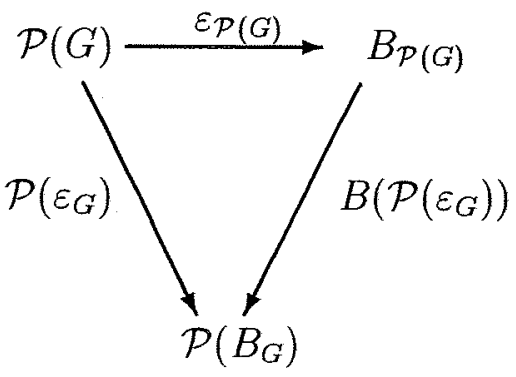

Já que a imagem de $\mathcal{P}(G)$ por $\varepsilon_{\mathcal{P}(G)}$ gera $B_{\mathcal{P}(G)}$ (3.48.(b)) e os morfimos no diagrama acima são injetores, concluímos que $B_{\mathcal{P}(G)}$ é isomorfa à subálgebra de Boole de $\mathcal{P}\left(B_{G}\right)$ gerada por 
$\mathcal{P}\left(\varepsilon_{G}\right)(\mathcal{P}(\mathrm{G}))$, como desejado.

Observação 6.20. Há outra prova de 6.19, um pouco mais técnica, mas que vale a pena registrar.

Seja $G$ um grupo especial reduzido e $\varepsilon_{G}: G \longrightarrow B_{G}$ a sua envoltória Booleana. Com notação análoga àquela em 5.39 , consideremos os sistemas cofiltrantes

$$
\left\{\begin{array}{cccc}
\mathcal{G} & =\left\langle G / \Delta ; p_{\Delta \Sigma}\right\rangle & & \Delta \subseteq \Sigma \text { em } \mathcal{F}(G) ; \\
B(\mathcal{G}) & =\left\langle B(G / \Delta) ; B\left(p_{\Delta \Sigma}\right)\right\rangle & & \Delta \subseteq \Sigma \text { em } \mathcal{F}(G) ; \\
\mathcal{B}_{\mathcal{G}} & = & \left\langle B_{G} / I ; p_{I J}\right\rangle & I \subseteq J \operatorname{em} \mathcal{F}\left(B_{G}\right),
\end{array}\right.
$$

onde o primeiro é o sistema cofiltrante associado a $\mathcal{P}(G)$, o segundo é obtido do primeiro pela aplicação do funtor envoltória Booleana (3.48.(e)), enquanto que o terceiro é o sistema projetivo associado a $\mathcal{P}\left(B_{G}\right)$. Sejam

$$
\left\langle\mathcal{P}(G) ; \mathfrak{p}_{\Delta}\right\rangle=\lim \mathcal{G}, \quad\left\langle\widehat{B} ; \beta_{\Delta}\right\rangle=\lim B(\mathcal{G}) \text { e }\left\langle\mathcal{P}\left(B_{G}\right) ; \mathfrak{p}_{I}\right\rangle=\lim \mathcal{B}_{\mathcal{G}} .
$$

Pela Proposição 6.10.(c), o par dual

$$
\varepsilon_{G *}: \mathcal{F}(G) \longrightarrow \mathcal{F}\left(B_{G}\right) \quad \text { e } \quad \varepsilon_{G}^{*}: \mathcal{F}\left(B_{G}\right) \longrightarrow \mathcal{F}(G)
$$

são funções crescentes e estabelecem correspondências biunívocas e inversas entre $\mathcal{F}(G)$ e $\mathcal{F}\left(B_{G}\right)$.

Fato 6.21. Se $I$ é um ideal de índice finito em $B_{G}$ e $\Delta=\varepsilon_{G}^{*}(I)$, existe um $\underline{\text { Ba-isomorfismo }}$

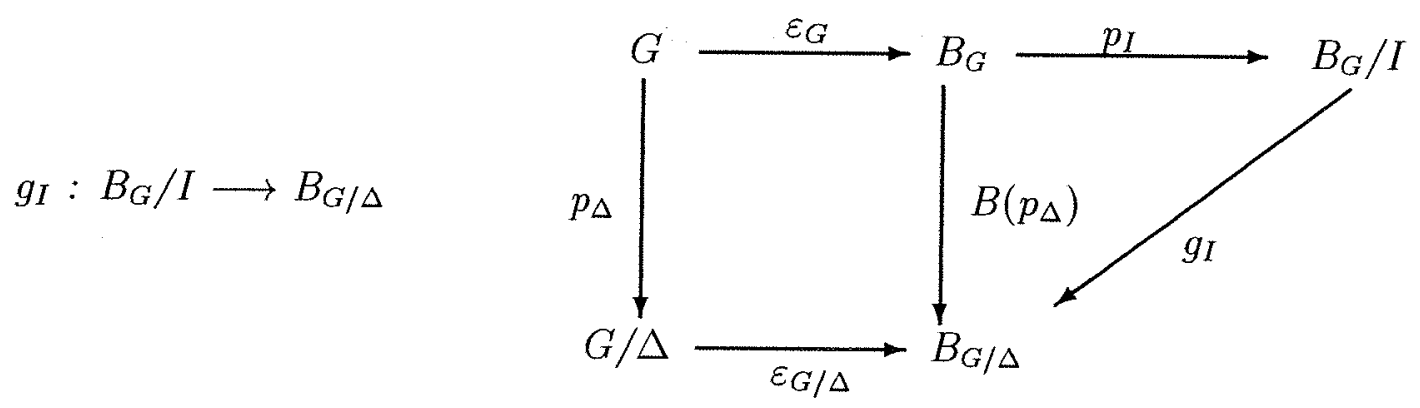

tal que o diagrama acima é comutativo.

Prova. O quadrado no diagrama acima é o característico da envoltória Booleana (3.48.(c)). Observe que como $\varepsilon_{(\cdot)}$ são injetoras, temos

$$
\varepsilon_{G}^{*}\left(\operatorname{ker} B\left(p_{\Delta}\right)\right)=p_{\Delta}^{-1}(\{1\})=\Delta .
$$

Como $I$ é um ideal de índice finito em $B_{G}$ temos que $\Delta=\varepsilon_{G}^{*}(I)$ é um subgrupo saturado de índice finito em $G$, logo $G / \Delta$ é um grupo especial reduzido finito. Segue que $B_{G / \Delta}$ é uma álgebra booleana finita e portanto o núcleo do Ba-morfismo $B\left(p_{\Delta}\right): B_{G} \longrightarrow B_{G / \Delta}$ é um ideal de indice finito em $B_{G}$.

Combinando as informações acima com a Proposição 6.10.(b) obtemos:

$$
I=\varepsilon_{G *}(\Delta)=\varepsilon_{G_{*}}\left(\varepsilon_{G}^{*}\left(\operatorname{ker} B\left(p_{\Delta}\right)\right)=\operatorname{ker} B\left(p_{\Delta}\right) .\right.
$$


Assim, o Teorema fundamental dos Ba-morfismos garante que existe um único Ba-isomorfismo, $g_{I}: B_{G} / I \longrightarrow B_{G / \Delta}$, que faz o triângulo à direita do diagrama do enunciado comutativo, como necessário.

Da Proposição 6.10.(c) e do Fato 6.21 concluímos que as associações:

*I $\in \mathcal{F}\left(B_{G}\right) \longmapsto \varepsilon_{G}^{*}(I) \in \mathcal{F}(G)$;

* Para cada $I \in \mathcal{F}\left(B_{G}\right), g_{I}: B_{G} / I \longrightarrow B(G / \Delta)$, onde $\Delta=\varepsilon_{G}^{*}(I)$;

constituem um isomorfismo de troca de base, $\alpha: \mathcal{B}_{\mathcal{G}} \longrightarrow B(\mathcal{G})$.

Uma ligeira variante da Proposição 2.38 (que está enunciada para a função identidade no lugar de uma bijeção crescente) garante que

$$
g=\lim _{\amalg}: \mathcal{P}\left(B_{G}\right) \longrightarrow \widehat{B}
$$

é um isomorfismo de álgebras de Boole. Pela própria definição de $g$, sabemos ser o único SGmorfismo tal que para todo $I \in \mathcal{F}\left(B_{G}\right)$ e $\Delta=\varepsilon_{G}^{*}(I)$, faz comutar o quadrado do diagrama abaixo :

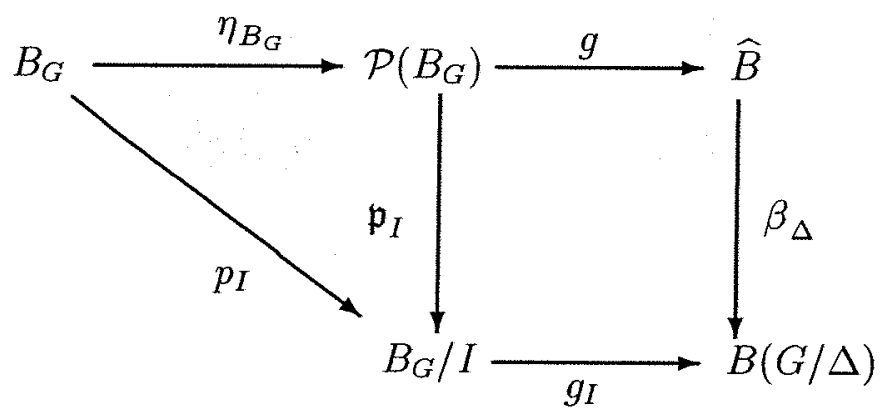

$$
\left(\begin{array}{c}
I=\varepsilon_{G *}(\Delta) \\
\mathrm{e} \\
\Delta=\varepsilon_{G}^{*}(I) .
\end{array}\right)
$$

Está claro que o diagrama inteiro comuta, pois $p_{I}=\mathfrak{p}_{I} \circ \eta_{B_{G}}$ é uma das propriedades básicas a envoltória profinita. Observe que $g$ é contínua. De fato, como

$$
\left\{\operatorname{ker} \mathfrak{p}_{I}: I \in \mathcal{F}\left(B_{G}\right)\right\} \quad \text { e } \quad\left\{\operatorname{ker} \beta_{\Delta}: \Delta \in \mathcal{F}(G)\right\}
$$

são pf-sistemas em $\mathcal{P}\left(B_{G}\right)$ e $\widehat{B}$, respectivamente, dado $\Delta \in \mathcal{F}(G)$, a comutatividade do diagrama (II) mostra que se tomarmos $I=\varepsilon_{*}(\Delta)$, então

$$
g\left(\operatorname{ker} \mathfrak{p}_{I}\right) \subseteq \operatorname{ker} \beta_{\Delta}
$$

garantindo a continuidade de $g$.

Pela construção em 5.39 e a Proposição 5.40.(a), temos um a SG-imersão completa e contínua

$$
h=\lim _{\leftarrow} \varepsilon_{G / \Delta}: \mathcal{P}(G) \longrightarrow \widehat{B} .
$$

Como acima, $h$ é o único SG-morfismo de $\mathcal{P}(G) \longrightarrow \widehat{B}$ tal que para todo $\Delta \in \mathcal{F}(G)$ o quadrado no diagrama abaixo é comutativo. 
(III)

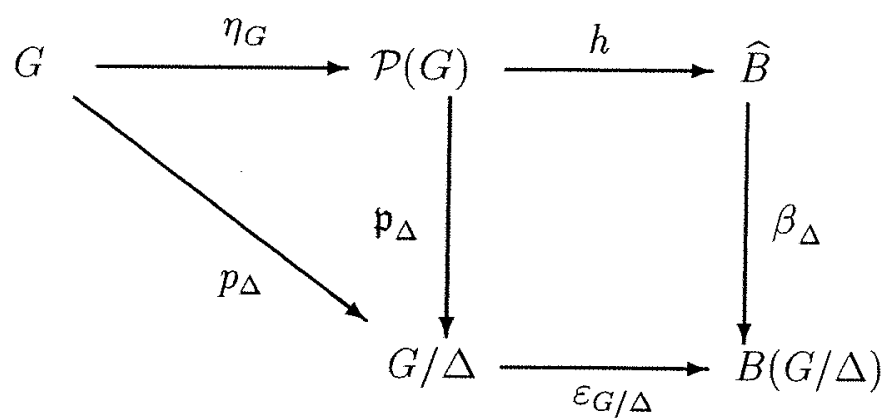

Note que o o diagrama inteiro é comutativo, pois o triângulo à esquerda vem da definição de $\mathcal{P}(G)$. Mostraremos que o triângulo

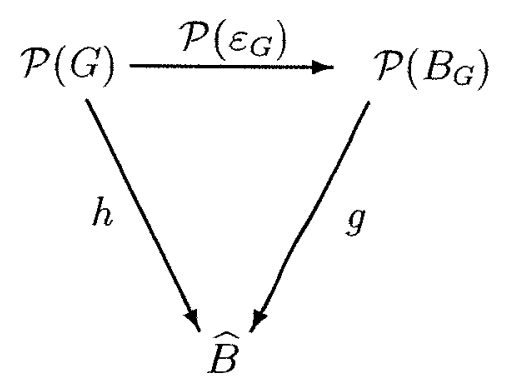

é comutativo. Tendo em vista a propriedade universal de $h=\lim \varepsilon_{G / \Delta}$ mencionada acima, a comutatividade de (IV) é equivalente a verificar que para todo $\overleftarrow{\Delta} \in \mathcal{F}(G)$, o quadrado no diagrama abaixo é comutativo ${ }^{11}$ :

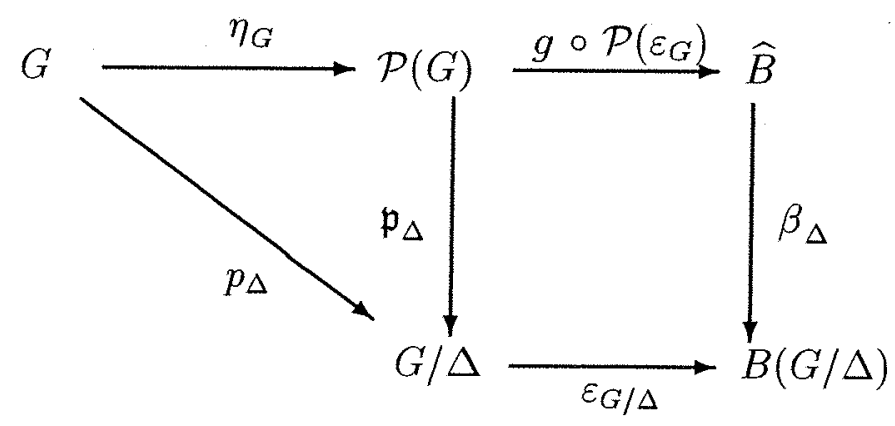

Dado $\Delta \in \mathcal{F}(G)$, utilizando a comutatividade do diagrama (II) e daquele no enunciado do Fato $6.21, \operatorname{com} I=\varepsilon_{G *}(\Delta)$, obtemos

$$
\begin{aligned}
\beta_{\Delta} \circ g \circ \mathcal{P}\left(\varepsilon_{G}\right) \circ \eta_{G} & =\beta_{\Delta} \circ g \circ \eta_{B_{G}} \circ \varepsilon_{G}=g_{I} \circ p_{I} \circ \varepsilon_{G}=B\left(p_{\Delta}\right) \\
& =\varepsilon_{G / \Delta} \circ p_{\Delta}=\varepsilon_{G / \Delta} \circ \mathfrak{p}_{\Delta} \circ \eta_{G} .
\end{aligned}
$$

Uma vez que a imagem de $\eta_{G}$ é densa em $\mathcal{P}(G)$ e as composições

$$
\beta_{\Delta} \circ g \circ \mathcal{P}\left(\varepsilon_{G}\right) \text { e } \varepsilon_{G / \Delta} \circ \mathfrak{p}_{\Delta}
$$

são funções contínuas, a igualdade em (VI) implica a comutatividade do quadrado em (V) e, consequentemente, do triângulo em (IV).

\footnotetext{
${ }^{11}$ Note que trata-se do mesmo diagrama que em (III), com $g \circ \mathcal{P}\left(\varepsilon_{G}\right)$ no lugar de $h$.
} 
Com estes preliminares enunciamos o

Teorema 6.22. Seja $\varepsilon_{G}: G \longrightarrow B_{G}$ a envoltória Booleana do grupo reduzido $G$. Com a notação em 6.20, existe um Ba-isomorfismo natural

$$
g: \mathcal{P}\left(B_{G}\right) \longrightarrow \widehat{B}=\lim _{\leftarrow} B(G / \Delta)
$$

tal que $g \circ \mathcal{P}\left(\varepsilon_{G}\right)=\underline{\lim } \varepsilon_{G / \Delta}$. Em particular

a) $\mathcal{P}\left(\varepsilon_{G}\right)$ é uma imersão completa e contínua.

b) Se identificarmos $\mathcal{P}\left(B_{G}\right)$ com $\widehat{B}$ via g, então $\mathcal{P}\left(\varepsilon_{G}\right)=\stackrel{\lim }{\leftarrow} \varepsilon_{G / \Delta}$.

Prova. Vem imediatamente do argumento apresentado na Observação 6.20 , lembrando que a Proposição 5.40 garante que $h=\lim \varepsilon_{G / \Delta}$ é uma imersão completa.

Agora podemos enunciar o

Teorema 6.23. O funtor $\mathcal{P}$ preserva e reflete imersões completas.

Prova. Seja $f: G \longrightarrow H$ uma imersão completa de grupos especiais reduzidos. Sabemos de 3.48.(e) que o quadrado abaixo a esquerda é comutativo,
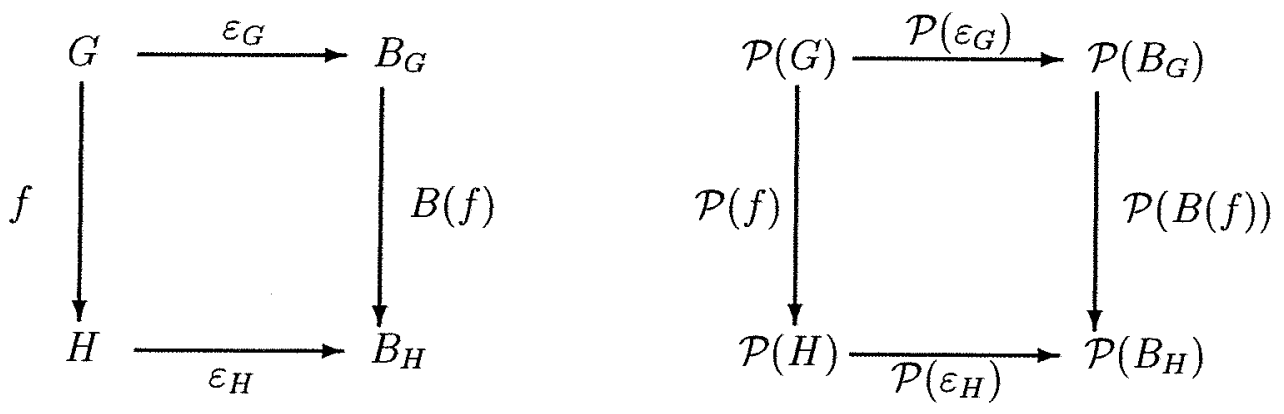

onde $B(f)$ é um Ba-morfismo injetor pelo item (3) do Teorema 5.2 em [DM1]. Aplicando o funtor $\mathcal{P}$ ao diagrama da esquerda, obtemos o diagrama comutativo acima a direita. Uma vez que $B(f)$ é um Ba-morfismo injetor, o Teorema 6.16.(b) garante que $\mathcal{P}(B(f))$ é uma imersão pura (e portanto completa). Como $\mathcal{P}\left(\varepsilon_{G}\right)$ também é uma imersão completa $(6.19,6.22)$, segue imediatamente que $\mathcal{P}(f)$ é uma imersão completa, como desejado.

Se $f: G \longrightarrow H$ é um SG-morfismo tal que $\mathcal{P}(f)$ é uma imersão completa, segue imediatamente do fato que $\eta_{G}$ reflete sub-formas (5.43.(a)) e da comutatividade do diagrama característico da envoltória profinita (5.52), isto é, 


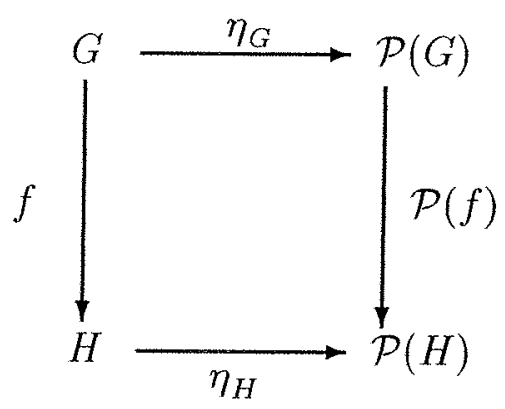

que $f$ é uma imersão completa, mostrando que $\mathcal{P}$ reflete imersões completas. 


\section{Referências Bibliográficas}

[AR] J. Adámek, J. Rosický, Locally Presentable and Accessible Categories, Lecture Notes Series 189, London Mathematical Society, Cambridge University Press, Cambridge, Great Britain, 1994.

[AKM] A. Adem, D. B. Karagueuzian, J. Mináč, On the Cohomology of Galois Groups Determined by Witt Rings, Advances in Mathematics 148 (1999), 105-160.

[AEJ] J. K. Aranson, R. Elman, B. Jacob, Rigid Elements, Valuations, and Realization of Witt Rings, Journal of Algebra 110 (1987), 449-467.

[Ast1] V. Astier, Théorie des modèles des groupes spéciaux de longueur de châ̂ne finie, Thèse de doctorat, Université Paris VII, Paris, France, 1999.

[Ast2] V. Astier, Pfister's subforms theorem and easy consequences, 2000, a aparecer.

[Ast3] V. Astier, Some model-theoretic results in the algebraic theory of quadratic forms Annals of Pure and Applied Logic, 112 (2001), 189-223.

[AT] V. Astier, M. Tressl, Axiomatization of local-global principles for $p p$-formulas in spaces of orderings, 2002, a aparecer.

[AM] M. F. Atiyah, I. G. Macdonald, Introduction to commutative algebra, AddisonWesley Series in Mathematics, Addison-Wesley Publishing Company, Great Britain, 1969.

[BD] R. Balbes, Ph. Dwinger, Distibutive Lattices, Univ. of Missouri Press, Columbia, Missouri, 1974.

[BS] J. L. Bell, A. B. Slomson, Models and Ultraproduts: an Introduction, NorthHolland Publishing Company, Amsterdan, Netherlands, 1971.

[Bou] N. Bourbaki, General Topology, part 1, Elements of Mathematics, Addison-Wesley Publishing Company, Great Britain, 1966.

[Bu] D. Bushaw, Elements of General Topology, John Wiley and Sons, New York, 1963.

[CK] C. C. Chang, H. J. Keisler, Model Theory, segunda edição, North-Holland Publishing Company, Amsterdam, Netherlands, 1976. 
[Cra] T. C. Craven, Characterizing Reduced Witt Rings of Fields, Journal of Algebra 53 (1978), 68-77.

[CS] T. C. Craven, T. Smith, Formally real fields from a Galois theoretic perspective, Jounal of Pure and Applied Algebra 145 (2000), 19-36.

[DM1] M. Dickmann, F. Miraglia, Special Groups: Boolean-Theoretic Methods in the Theory of Quadratic Forms, Memoirs of the AMS 689, American Mathematical Society, Providence, USA, 2000.

[DM2] M. Dickmann, F. Miraglia, On Quadratic Forms whose total signature is zero mod $2^{n}$. Solution to a problem of M. Marshall, Inventiones mathematicae 133 (1998), 243-278.

[DM3] M. Dickmann, F. Miraglia, Lam's Conjecture, a aparecer no Algebra Colloquium, 2002.

[DM4] M. Dickmann, F. Miraglia, Algebraic K-theory of Special Groups, 2001, preprint.

[DM5] M. Dickmann, F. Miraglia, Bounds for the representation of quadratic forms, 2001; preprint.

[DM6] M. Dickmann, F. Miraglia, Elementary behaviour of the boolean hull functor, a aparecer no Journal of Symbolic Logic, 2002.

[DM7] M. Dickmann, F. Miraglia, Lattice-Ordered Reduced Special Groups, 2002, preprint.

[GJ] L. Gillman, M. Jerison, Rings of Continuous Functions, D, Van Nostrand Publ. Co., Princeton, N. J., 1960.

[GV] A. Grothendieck, J. L. Verdier, Préfaisceaux, Exposé I em SGA 4, Lecture Notes in Mathematics 269, Springer-Verlag, Berlin, Germany, 1972, 1-217.

[Ho] W. Hodges, Model Theory, Encyclopedia of Mathematics and its Applications, 42, Cambridge Univ. Press, Cambridge, U. K., 1993.

[Kl] S. C. Kleene, Introduction to Metamathematics, North-Holland Publ. Co., Amsterdam, 1952.

[K11] S. C. Kleene, Mathematical Logic, John Wiley and Sons, Inc., New York, 1967.

[KMS] M. Kula, M. A. Marshall, A. Sladek, Direct limits of finites spaces of orderings, Pacific Journal of Mathematics 112 (1984), 391-406.

[Lam1] T. Y. Lam, The algebraic theory of quadratic forms, W. A. Benjamin, Massachusetts, USA, 1973.

[Lam2] T. Y. Lam, Orderings, valuations and quadratic forms, Regional Conference Series in Mathematics 52, American Mathematical Society, Providence, USA, 1983.

[Lam3] T. Y. Lam, An introduction to real algebra, Rocky Mountain Jounal of Mathematics 14 (1984), 767-814. 
[Lim] A. L. de Lima, Les groupes spéciaux. Aspects Algébriques et Combinatoires de la Théorie des Espaces d'Ordres Abstraits, Thèse de doctorat, Université Paris VII, Paris, France, 1996.

[Mac] S. Mac Lane, Categories for the Working Mathematician, Graduated Texts in Mathematics 5, Springer-Verlag, New York, USA, 1971.

[MP] M. Makkai, R. Paré, Accessible Categories: The Foundations of Categorical Model Theory, Contemporary Mathematics 104, American Mathematical Society, Providence, USA, 1989.

[Mar1] M. A. Marshall, Abstract Witt Rings, Queen's Papers in Pure and Applied Mathematics 57, Queen's University, Ontario, Canada, 1980.

[Mar2] M. A. Marshall, Spaces of Orderings and Abstract Real Spectra, Lecture Notes in Mathematics 1636, Springer-Verlag, Berlin, Germany, 1996.

[Mar3] M. A. Marshall, Classification of Finite Spaces of Orderings, Canadian Journal of Mathematics 31 (1979), 320-330.

[Mar4] M. A. Marshall, Quocients and Inverse Limits of Spaces of Orderings, Canadian Journal of Mathematics 31 (1979), 604-616.

[Mar5] M. A. Marshall, Spaces of Orderings IV, Canadian Journal of Mathematics 32 (1980), 603-627.

[Mar6] M. A. Marshall, The Witt Ring of a Space of Orderings, Transaction of the AMS 258 (1980), 505-521.

[Mar7] M. A. Marshall, Open questions in the theory of spaces of orderings, Journal Of Symbolic Logic 67, 341-352, 2001.

[Mar8] M. A. Marshall, Spaces of Orderings and Abstract Real Spectra, Lecture Notes in Math. 1636, Springer-Verlag, Berlin, 1996.

[M] F. Miraglia, The Space of Satured Subgroups of Finite Index of a Reduced Special Group, 2000, preprint.

[Mit] B. Mitchell, Theory of Categories, Academic Press, N. York, 1965.

[Pfi] A. Pfister, Quadratische Formen in beliebigen Körpern, Inventiones Matematicae 1 (1966), 116-132.

[Pr] D. Prawitz, Natural Deduction, Almquist, Stockholm, 1976.

[Ra] H. Rasiowa, An Algebraic Approach to Non-Classical Logics, North-Holland Publ. Co., Amsterdam, 1974.

[RS] H. Rasiowa, R. Sikorski, The Mathematics of Metamathematics, Polish Academy od Sciences Publ., 41 (segunda edição), Warsóvia, 1968. 
[Rib] L. Ribes, Introduction to Profinite Groups and Galois Cohomology, Queen's Papers in Pure and Applied Mathematics 24, Queen's University, Ontario, Canada, 1970.

[Sch] J. R. Schoenfield, Mathematical Logic, Addison-Wesley Publ. Co., Reading, Mass., 1967.

[Sc] D. S. Scott, Identity and Existence in Intuitionistic Logic, in Applications of Sheaves, Springer Lecture Notes in Math., 753, 660-696, 1979.

[Sm] R. Smulian, First-Order Logic, Springer-Verlag, Berlin, 1978.

[Tu] J. W. Tukey, Convergence and Uniformity in Topology, Princeton University Press, Princeton, N. J., 1940. 


\title{
Índice Remissivo
}

\author{
$G[\Delta], 101$ \\ $\operatorname{Sat}(G), 83,95$ \\ $\operatorname{Ssat}(G), 94$ \\ $\operatorname{Ssat}_{\Theta}^{\star}(G), 120$ \\ $\operatorname{Ssat}_{\Theta}(G), 120$ \\ $\operatorname{Ult}(I, \leq), 20$ \\ $X(G), 120$ \\ $X(f), 187$ \\ $X_{\Theta}(G), 120$ \\ $\operatorname{Diag}_{\Omega}(\mathcal{C}), 40$ \\ $\overleftarrow{\Delta(M)}, 52$ \\ $\bigvee S, 104$ \\ $\bigwedge S, 104$ \\ $\mathcal{F}(G), 120$ \\ $\mathcal{F}_{\Theta}(G), 120$ \\ $\mathcal{P}(G), 154$ \\ $\mathcal{T}(M), 52$ \\ $\mathcal{W}(G), 111$ \\ $\chi(G), 188$ \\ $\Delta^{+}(M), 52$ \\ $\exists^{+}(L), 49$ \\ $S_{\text {fin }}(G), 107$ \\ $\operatorname{cl}(G), 106$ \\ $\mathbf{R S G}_{c l f}, 107$ \\ Pfister $(A), 94$ \\ $\operatorname{dim} \varphi, 82$ \\ $\operatorname{pp}(L), 49$ \\ $D_{H}(\varphi), 82$ \\ $\operatorname{Ssat}^{\star}(G), 120$ \\ à, 193 \\ $f^{*}, 114$ \\ $\dot{F}, \dot{F}^{2}, \Sigma \dot{F}^{2}, 102$ \\ $\operatorname{ind}_{W}(\varphi), 83$ \\ $f_{*}, 114$ \\ $2^{A}, 2_{\text {fin }}^{A}, 123$ \\ $X_{G}^{+}, 107$ \\ $f \star \varphi, 88$ \\ $X_{a}, 187$
}

$\eta_{G}, 154$

$\varphi_{a n}, 84$

$\mathfrak{c}_{A}, 193$

$\rho_{f}, 193$

$\subseteq_{f}, 188$

Ba, 104

PSG, 88

RSG, 88

SG, 88

pif, 20

pst, 146

álgebra de Boole, 104 completa, 149

anel de Witt, 111

graduado de Witt, 111

assinatura, 89

cálculo de predicados

clássico, 48

intuicionista, 47

carácter (SG-), 88

categoria, 21

cofiltrada, 136

filtrada, 136

Conjectura

de Marshall, 111

conjunto

consistente, 48

inconsistente, 48

ordenado, 19

dirigido, 20

corpo

formalmente real, 102

Pitagórico, 102

diagrama, 52

completo, 52 
positivo, 52

dualidade

de Stone, 187

envoltória

Booleana, 105

profinita, 154

equivalência.

de Witt, 110

elementar, 51

espaço

de ordens, 88

extensão (de um grupo especial), 101

fórmula

$\boldsymbol{\Pi}_{\boldsymbol{n}}, 47$

$\mathbf{\Sigma}_{n}, 47$

existencial, 46

existencial positiva, 47

Horn, 47

positiva primitiva, 47

fan, 101

filtro (em uma álgebra de Boole), 104

forma, 82

anisotrópica, 83

de Pfister, 83

dimensão de uma,- 82

discriminante de uma,- 83

hiperbólica, 83

isotrópica, 83

parte anisotrópica, 84

sub-, 83

funtor

de Stone, 187

que preserva (co)limites, 37

que preserva imersões elementares, 72

que preserva imersões puras, 72

que preserva ultrapotências, 71

grupo

especial, 82

de comprimento de cadeia finito, 107

quociente, 95

representável, 103

pré-especial, 81

topológico, 125

Booleano, 125 discreto, 125

Hausdorff, 125

ideal

fundamental, 111

ideal maximal, 104

imagem

inversa saturada, 114

saturada, 114

imersão

$L-, 51$

canônica (em $\mathcal{P}(G), 154$

elementar, 51

imersão completa, 89

índice de Witt, 83

isometria, 82

isomorfismo, 24

limite

indutivo

filtrante, 38

indutivo ou colimite, 37

projetivo

cofiltrante, 38

projetivo ou limite, 37

maximal

filtro, ideal, 104

morfismo

$L-, 51$

de álgebras de Boole, Ba-, 104

completo, 149

de grupos especiais, 88

de grupos pré-especiais, 87

de troca de base, 147

derivado, 116

diagonal, 56

iso-, 24

puro, 90

que reflete índice de Witt, 89

que reflete isotropia, 89

que reflete subformas, 89

regular, 96

objeto

final, 25

inicial, 25 
pf-sistema, 137

produto

de $L$-estruturas, 53

de formas, 83

de grupos especiais, 100

reduzido, 55

puro

$L$-morfismo, 67

representação, 82

saturado, 94

seção (de um grupo especial), 107

soma

de formas, 83

subforma, 83

subgrupo

completo, 91

especial, 91

pré-especial, 91

puro, 91

\section{Teorema}

da Completude e Compacidade, 51

da Dedução, 48

teorema, 48

translação (em um grupo), 125

ultrafiltro (em uma álgebra de Boole), 104

ultrapotência, 55

ultraproduto, 55 